RICARDO NOZAKI

\title{
ANÁLISE DE SÉRIES TEMPORAIS COM COMPORTAMENTO NÃO LINEAR OBTIDAS POR UM SENSOR DE UM MICROSCÓPIO DE FORÇA ATÔMICA
}

Tese apresentada à Escola de Engenharia de São Carlos da Universidade de São Paulo, como parte dos requisitos para obtenção do título de Doutor em Ciências.

Área de Concentração: Dinâmica de Máquinas e Sistemas

Orientador: Prof. Dr. Hélio Aparecido Navarro

ESTE EXEMPLAR TRATA-SE DA VERSÃO CORRIGIDA. A VERSÃO ORIGINAL ENCONTRA-SE DISPONÍVEL JUNTO AO DEPARTAMENTO DE ENGENHARIA MECÂNICA DA EESC-USP

São Carlos

2016 
AUTORIZO A REPRODUĈ̃O TOTAL

OU PARCIAL DESTE TRABALHO, POR

QUALQUER MEIO CONVENCIONAL OU

ELETRÔNICO, PARA FINS DE ESTUDO

E PESQUISA, DESDE QUE CITADA A FONTE.

N961

Nozaki, Ricardo

ANÁLISE DE SÉRIES TEMPORAIS COM COMPORTAMENTO NÃO LINEAR OBTIDAS POR UM SENSOR DE UM MICROSCÓPIO DE FORÇA ATÔMICA / Ricardo Nozaki; orientador Hélio Aparecido Navarro . São Carlos, 2016.

Tese (Doutorado) - Programa de Pós-Graduação em Engenharia Mecânica e Área de Concentração em Materiais -- Escola de Engenharia de São Carlos da Universidade de São Paulo, 2016.

1. AFM. 2. caos. 3. séries temporais. 4. teste $0-1$. 5. identificação de sistemas. 6. reconstrução do espaço de estados. I. Título. 


\section{FOLHA DE JULGAMENTO}

Candidato: Licenciado RICARDO NOZAKI.

Título da tese: "Análise de séries temporais com comportamento não linear obtidas por um sensor de um microscópio de força atômica".

Data da defesa: 21/10/2016.

\section{Comissão Julgadora:}

Prof. Dr. Helio Aparecido Navarro

(Orientador)

(Escola de Engenharia de São Carlos/EESC)

Prof. Dr. Daniel Varela Magalhães

(Escola de Engenharia de São Carlos/EESC)

Prof. Dr. Angelo Marcelo Tusset

(Universidade Tecnológica Federal do Paraná/UTFPR)

Prof. Dr. Marcelo de Assumpção Pereira da Silva

(Centro Universitário Central Paulista/UNICEP)

Prof. Dr. Arlindo Neto Montagnoli

(Universidade Federal de São Carlos/UFSCar)
Resultado:

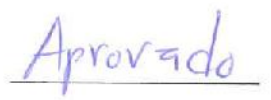

APnOUADO
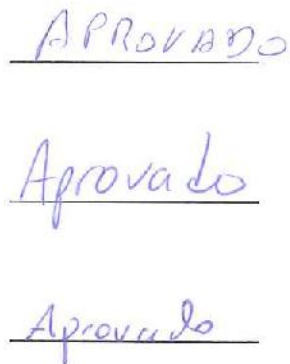

Coordenador do Programa de Pós-Graduação em Engenheira Mecânica: Prof. Associado Gherhardì Ribatski

Presidente da Comissão de Pós-Graduação: Prof. Associado Paulo César Lima Segantine 


\section{DEDICATÓRIA}

Aos meus pais que sempre me apoiaram, incentivaram e foram fontes de toda minha inspiração dessa vida. 


\section{AGRADECIMENTOS}

Agradeço intensamente aos meus pais Junkiti e Tsuguiko, a minha família, ao professor Dr. Hélio Aparecido Navarro, ao Prof. Dr. José Manoel Balthazar, aos amigos não lineares pela ajuda nos programas e a CAPES pelo financiamento. 


\section{RESUMO}

NOZAKI, R. Análise de séries temporais com comportamento não linear obtidas por um sensor de um microscópio de força atômica. 2016. 123p. Tese (Doutorado) Escola de Engenharia de São Carlos, Universidade de São Paulo, São Carlos, 2016.

O estudo das não linearidades das séries temporais da microviga de um microscópio de força atômica tem sido essencial para o desenvolvimento e aperfeiçoamento deste equipamento de ampliação de imagens. Não linearidades podem aparecer com frequência nos experimentos, afetando significativamente a resposta prevista de forma que estas instabilidades se concretizam em imagens ruins. Esta tese apresenta resultados obtidos através de uma abordagem experimental e teórica. Buscou-se aperfeiçoar modelos clássicos de osciladores do microscópio de força atômica melhorando seu comportamento caótico através da observação dos resultados dos experimentos. A identificação de sistemas é feita pelo método de espaço de estados. Outra abordagem de séries temporais obtidas através de um microscópio de força atômica torna possivel a reconstrução de espaço de estados, utilizando-se de técnicas como informação mútua, falsos vizinhos e defasagem de tempo. Analisa-se também o comportamento caótico das séries temporais usando o teste 0-1 e escala indexada em quatro experimentos que resultam em um mapa que relaciona a altura que a microviga vibra com o coeficiente do teste 0-1 e com a escala indexada.

Palavras chave: Microscopia de força atômica, séries temporais, identificação de sistemas, caos, teste 0-1. 


\begin{abstract}
Nozaki, R. Time series analysis with nonlinear behavior obtained by a sensor of an atomic force microscope. 2016. 123p. Thesis (Phd) - Escola de engenharia de São Carlos, Universidade de São Paulo, São Carlos, 2016.

The study of time series nonlinearities of the cantilever's atomic force microscope has been essential to the development and improvement of this image magnification equipment. Nonlinearities may appear frequently in the experiments, significantly affecting expected response in a way that these instabilities generate bad images. This thesis presents results obtained through an experimental and theoretical approach accordingly. We attempted to improve the classical models of oscillators atomic force microscope improving its chaotic behavior by observing the results of the experiments. The system identification is made by method space state. Another approach to time series obtained through an atomic force microscope makes it possible to reconstruct space phase, using techniques such as mutual information and false neighbors delay. It is analyzed chaotic behavior time series by using the 0-1 test and scale index in four experiments resulting in a map that relates the height of cantilever deflections with the 0-1 test coefficient and the indexed scale.
\end{abstract}

Keywords: Atomic Force Microscope, time series, system identification, chaos, teste 0-1 


\section{LISTA DE ILUSTRAÇÕES}

Figura 2. 1 - Esquema de funcionamento do AFM adaptado de (SEBASTIAN et Al., 2007)

Figura 2. 2 - Esquema da ação do "micromicroviga" durante a operação em "AFM" Adaptado de (SEBASTIAN et Al., 2007)

Figura 2. 3 - Os regimes de força do AFM e os três modos de operar. Adaptado de Bowen (2009).

Figura 2. 4 - Tipos de ponteiras. Bowen (2009).

Figura 2. 5 - Constante elástica visualizada no programa nanoscope $\AA$

Figura 2. 6 - Dimensões da microviga do AFM

Figura 2. 7 - Modelo proposto por Jalili,et al. (2009).

Figura 2. 8 - Retrato de fase de Wang et Al. (2008)

Figura 3. 1 - Exemplo de série temporal estacionária

Figura 3. 2 - Série temporal não estacionária de www.portalaction.com.br

Figura 3. 3 - Figura de Viola (2011) - Possíveis falsos vizinhos próximos e suas dimensões de imersão.

Figura 4. 1 - Modelo da ponteira da microviga do AFM

Figura 4. 2 - O retrato de fase da equação modelada com 4000 pontos

Figura 4.3 - Esquema proposto por Viola (2011)

Figura 4. 4 - Informação mútua da série produzida pela equação modelada

Figura 4.5 - Porcentagem de falsos vizinhos calculada para a série produzida pela equação modelada

Figura 4. 6 - Retrato de fase da série temporal reconstruída

Figura 5. 1 - AFM trabalhando em modo 'soft tapping' nas condições de contato e não contato

Figura 5. 2 - Preparação da amostra

Figura 5. 3 - Visão ampliada do suporte que fixa o tip

Figura 5. 4 - AFM com o tip instalado

Figura 5. 5 - Calibração do microscópio de força atômica 
Figura 5. 6 - Trajeto em linha do escâner do AFM na amostra de safira

Figura 5. 7 - Relevo da imagem criada pelo modo contato em vermelho e as demais cores em modo não contato.

Figura $5.8-z=671.32 \mathrm{~nm} \quad 46$

Figura $5.9-z=634.65 \mathrm{~nm} \quad 47$

Figura $5.10-z=592.34 \mathrm{~nm} \quad 47$

Figura $5.11-z=507.64 \mathrm{~nm} \quad 48$

$\begin{array}{ll}\text { Figura } 5.12-\mathrm{z}=338.24 \mathrm{~nm} & 48\end{array}$

Figura $5.13-z=253.86 \mathrm{~nm} \quad 49$

Figura $5.14-z=50.24 \mathrm{~nm} \quad 49$

Figura 5. 15 - Histórico de deflexões para $z=622.2 \mathrm{~nm} \quad 50$

Figura 5. 16 - Histórico de deflexões para $z=605.2 \mathrm{~nm}$

Figura 5. 17 - Histórico de deflexões para $z=588.2 \mathrm{~nm} \quad 51$

Figura 5. 18 - Histórico de deflexões para $z=571.2 \mathrm{~nm}$

Figura 5. 19 - Histórico de deflexões para $z=554.2 \mathrm{~nm}$

Figura 5. 20 - Histórico de deflexões para $z=537.2 \mathrm{~nm} \quad 53$

Figura 5. 21 - Histórico de deflexões para $z=520.2 \mathrm{~nm}$

Figura 5. 22 - Histórico de deflexões para $z=503.2 \mathrm{~nm} \quad 54$

Figura 5. 23 - Histórico de deflexões para $z=486.2 \mathrm{~nm} \quad 54$

Figura 5. 24 - Histórico de deflexões para $z=401.2 \mathrm{~nm} \quad 55$

Figura 5. 25 - Série temporal do AFM em descida no modo tapping 56

Figura 5. 26 - Série temporal do AFM em descida no modo tapping 56

Figura 5. 27 - Série temporal do AFM em descida no modo tapping 57

Figura 5. 28 - Série temporal do AFM em descida no modo tapping 57

Figura 5. 29 - Série temporal do AFM em descida no modo tapping 57

Figura 5. 30 - Série temporal do AFM em descida no modo tapping 58

Figura 5. 31 - Série temporal do AFM em descida no modo tapping 58

Figura 5. 32 - Série temporal do AFM em descida no modo tapping 58

Figura 5. 33 - Série temporal do AFM em descida no modo tapping 59

Figura 5. 34 - Gráfico de variação de $K$ para série 'a' 61

Figura 5. 35 - Escala indexada para série 'a' 61

Figura 5. 36 - Informação mútua da série temporal da safira na altura $Z=10.2 \mathrm{~nm}$ com mínimo local em $\tau=13$ 
Figura 5. 37 - Porcentagem de falsos vizinhos próximos para a amostra de safira em $\mathrm{Z}=10.2 \mathrm{~nm}$ indica $m=5$

Figura 5. 38 - Deslocamento da microviga em $\mathrm{z}=10.2 \mathrm{~nm}$

Figura 5. 39 - Retrato de fase da série temporal em safira em $Z=10.2 \mathrm{~nm}$ reconstruída com $\mathrm{m}=5$ e $\tau=13$

Figura 5. 40 - Reconstrução de espaço da série de safira em $\mathrm{Z}=10.2 \mathrm{~nm}$ pelo método das derivadas

Figura 5. 41 - FFT da série temporal em safira na altura $Z=10.2 \mathrm{~nm}$

Figura 5. 42 - Informação mútua da série temporal da safira na altura $\mathrm{z}=316.2 \mathrm{~nm}$ com mínimo local em $\tau=10$

Figura 5. 43 - Dimensão da série em safira $Z=316.2 \mathrm{~nm}$ indica $m=5$ pelo método dos falsos vizinhos

Figura 5. 44 - Deslocamento da microviga em z=316.2nmm

Figura 5. 45 - Retrato de fase da série temporal em safira em Z=316.2nm reconstruída com $\mathrm{m}=5$ e $\tau=10$

Figura 5. 46 - Reconstrução do espaço de estados para a série em safira em Z=316.2nm pelo método das derivadas

Figura 5. 47 - FFT da série temporal em safira em $Z=316.2 \mathrm{~nm}$

Figura 5. 48 - Gráfico de variação de $K$ para série 'b'.

Figura 5. 49 - Escala indexada para a série 'b'

Figura 5. 50 - Informação mútua da série temporal da safira com ponta triangular na altura $\mathrm{z}=59.2 \mathrm{~nm}$ com mínimo local em $\tau=15$

Figura 5. 51 - Dimensão de imersão calculada para a série de safira com ponta triangular em $\mathrm{Z}=59.2 \mathrm{~nm}$ pelo método dos falsos vizinhos próximos

Figura 5. 52 - Deslocamento da microviga em $\mathrm{z}=59.2 \mathrm{~nm}$

Figura 5. 53 - Retrato de fase da série temporal em safira com ponta triangular em $\mathrm{Z}=59.2 \mathrm{~nm}$ reconstruída com $\mathrm{m}=5$ e $\tau=13$

Figura 5. 54 - Reconstrução do espaço de estados da série em safira com ponta triangular em $\mathrm{Z}=59.2 \mathrm{~nm}$ pelo método das derivadas

Figura 5. 55 - FFT da série de safira com ponta triangular em $Z=59.2 \mathrm{~nm}$

Figura 5. 56 - Informação mútua da série temporal da safira na altura $\mathrm{z}=553 \mathrm{~nm}$ com mínimo local em $\tau=10$

Figura 5. 57 - Dimensão de imersão calculado para a série analisada em safira com ponta triangular em $\mathrm{Z}=553 \mathrm{~nm}$ pelo método dos falsos vizinhos 
Figura 5. 58 - Deslocamento da microviga em $\mathrm{z}=553 \mathrm{~nm}$

Figura 5. 59 - Retrato de fase da série temporal em safira com ponta triangular em $\mathrm{Z}=553 \mathrm{~nm}$ reconstruída com $\mathrm{m}=5$ e $\tau=13$

Figura 5. 60 - Retrato de fase da safira com ponta triangular em Z=553nm reconstruído

pelo método das derivadas

Figura 5. 61 - FFT da série produzida em safira com ponta triangular e Z=553nm 78

Figura 5. 62 - Gráfico de variação de $K$ para série 'c’ 80

Figura 5. 63 - Teste da escala indexada para a série 'c' 80

Figura 5. 64 - Informação mútua da série temporal analisada em borracha na altura $\mathrm{Z}=306 \mathrm{~nm}$ com mínimo local em $\tau=8$

Figura 5. 65 - Porcentagem de falsos vizinhos para a série analisada em borracha na altura $\mathrm{Z}=306 \mathrm{~nm}$ com $m=5$.

Figura 5. 66 - Deslocamento da microviga em $\mathrm{z}=306 \mathrm{~nm}$

Figura 5. 67 - Retrato de fase da série temporal analisada em borracha com Z=306nm reconstruída com $m=5$ e $\tau=8$

Figura 5. 68 - Reconstrução do espaço de estados pelo método das derivadas da série analisada em borracha com Z=306nm

Figura 5. 69 - FFT da série produzida em borracha com Z=306nm

Figura 5.70 - Informação mútua da série temporal analisada em borracha na altura

$\mathrm{Z}=382.2 \mathrm{~nm}$ com mínimo local em $\tau=10$

Figura 5. 71 - Porcentagem de falsos vizinhos para a série analisada em borracha na altura $Z=382.2 \mathrm{~nm}$ com $m=5$.

Figura 5. 72 - Deslocamento da microviga em $\mathrm{z}=382.2 \mathrm{~nm}$

Figura 5.73 - Retrato de fase da série temporal em borracha com Z=382.2 nm reconstruída com $m=5$ e $\tau=13$

Figura 5. 74 - Retrato de fase reconstruída com série analisada em borracha em $\mathrm{Z}=382.2 \mathrm{~nm}$ pelo método das derivadas

Figura 5. 75 - FFT da série temporal analisada em borracha com $Z=382.2 \mathrm{~nm}$. $\quad 88$

Figura 5.76 - Variação de $K$ do teste 0-1 para série 'd' 89

Figura 5. 77 - Teste da escala indexada para a série 'd' 89

Figura 5.78 - Informação mútua da série temporal analisada em safira no nitrogênio na altura $\mathrm{Z}=40 \mathrm{~nm}$ com mínimo local em $\tau=12$ 
Figura 5. 79 - Porcentagem de falsos vizinhos para a série analisada na safira com nitrogênio na altura $Z=40 \mathrm{~nm}$ com $m=5$.

Figura 5. 80 - Deslocamento da microviga em $\mathrm{z}=40 \mathrm{~nm}$

Figura 5. 81 - Retrato de fase da série temporal em safira com nitrogênio com Z=40nm reconstruída com $m=5$ e $\tau=12$

Figura 5. 82 - Retrato de fase reconstruído da série analisada na safira em nitrogênio

pelo método das derivadas com $\mathrm{Z}=40 \mathrm{~nm}$

Figura 5. 83 - FFT da série temporal analisada em safira com nitrogênio com Z=40nm

Figura 5. 84 - Informação mútua da série temporal analisada em safira no nitrogênio na altura $\mathrm{Z}=553 \mathrm{~nm}$ com mínimo local em $\tau=9$

Figura 5. 85 - Dimensão $m=5$ calculado pelo algoritmo de falso vizinhos próximos para a série analisada em safira no nitrogênio com $Z=553 \mathrm{~nm}$

Figura 5. 86 - Deslocamento da microviga em $\mathrm{z}=553 \mathrm{~nm}$

Figura 5. 87 - Retrato de fase da série temporal z=553 nm em safira no nitrogênio reconstruída com $m=5$ e $\tau=9$

Figura 5. 88 - Reconstrução de espaço de estados pelo método das derivadas na série de safira no nitrogênio em $Z=553 \mathrm{~nm}$

Figura 5. 89 - FFT para série em safira no nitrogênio com Z=553nm

Figura 5. 90 - Gráfico de testes 0-1 para série 'a', 'b', 'c', e'd'

Figura 5. 91 - Teste escala indexada com as séries 'a', 'b', 'c' e 'd'.

Figura 6. 1 - Em pontilhado preto a série temporal para $\mathrm{z}=537 \mathrm{~nm}$ e em vermelho a série identificada pelo método de espaço de estados.

Figura 6. 2 - Esquema de identificação de sistemas (LJUNG, 1999).

Figura 6. 3 - Em cor azul a série temporal identificada com $\mathrm{z}=537.2 \mathrm{~nm}$ deflexão da microviga em meio líquido e em amarela a série com z=537.2 nm no ar

Figura 6. 4 - Exemplo de região da série temporal onde não há identificação de sistemas 


\section{LISTA DE TABELAS}

Tabela 1- Informação mútua para série obtida pelo modelo AFM

Tabela 2- Tabela com teste $0-1$ e escala indexada da série ' $a$ '

Tabela 3 - informação mútua da série A em z=10.2nm

Tabela 4 - Informação mútua para série A em z=316.2nm

Tabela 5 - Tabela com teste 0 -1 e escala indexada para série ' $b$ '

Tabela 6 - Informação mútua para série B em z=59.2nm

Tabela 7 - Informação mútua para a série $\mathrm{B}$ em z=553nm

Tabela 8 - Tabela com teste 0 - 1 e escala indexada para série 'c'

Tabela 9 - Informação mútua para a série $\mathrm{C}$ em z=306nm 81

Tabela 10 - Informação mútua da série C em z=382nm 85

Tabela 11 - Tabela com teste 0-1 e escala indexada aplicada na série 'd' 88

Tabela 12 - Informação mútua para série D em z=40nm 90

Tabela 13 - Informação mútua para série D em z=553nm 93

Tabela 14 - Séries temporais e suas respectivas validações 103 


\section{LISTA DE SIGLAS}

AFM

FNN

ODE

ddp

$F_{v d w}$
$I$
$K_{n l}$
$K_{l}$
$\tau$
$D$
$m$
$z$
$x$
$f$
$S(n)$
$U(n)$
$K$

LISTA DE SÍMBOLOS

Microscópio de Força atômica

Falsos Vizinhos Próximos

Equação diferencial ordinária

diferença de potencial

Força de Van der Waals

Informação mútua

Constante elástica não linear

Constante elástica linear

Atraso

Dimensão

dimensão de imersão

altura em que a microviga está fixada

deflexão da microviga

força

série temporal

série reconstruída

coeficiente do teste $0-1$ 


\section{SUMÁRIO}

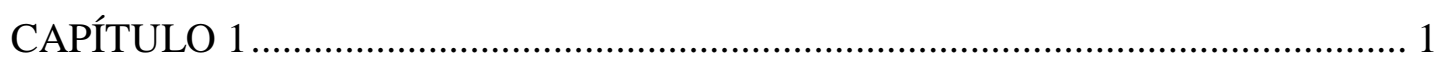

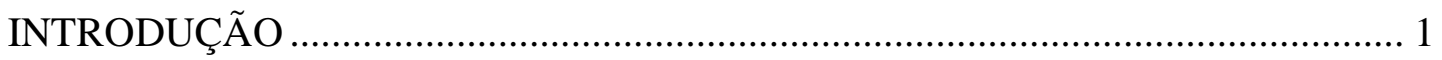

1.2 APRESENTAÇÃO DOS CAPÍTULOS .......................................................... 4

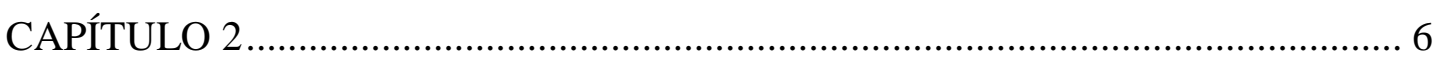

REVISÃO BIBLIOGRÁFICA ............................................................................ 6

DESCRIÇÃO DO PROBLEMA AFM................................................................. 6

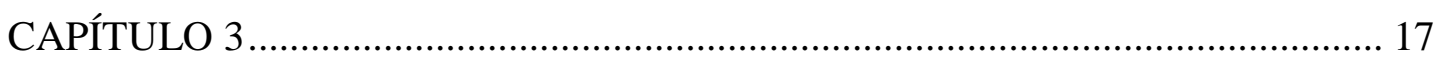

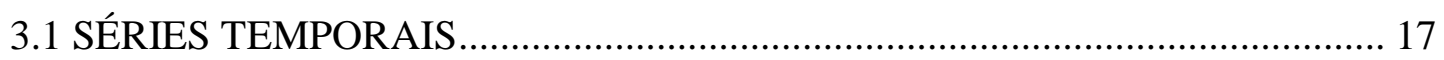

3.2 RECONSTRUÇÃO DO ESPAÇO DE ESTADOS ………………………... 19

3.3 MÉTODO DA INFORMAÇÃO MÚTUA MÉDIA ……………………….... 20

3.4 MÉTODO DOS FALSOS VIZINHOS PRÓXIMOS ……………………...... 21

3.5 IDENTIFICAÇÃO DE SISTEMAS ……………………………………..... 22

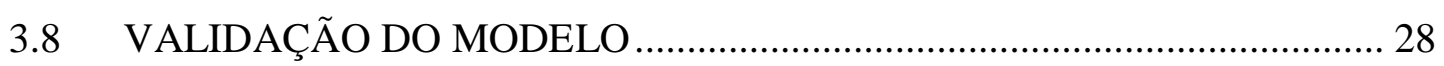

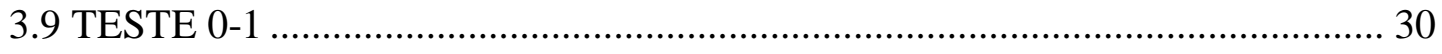

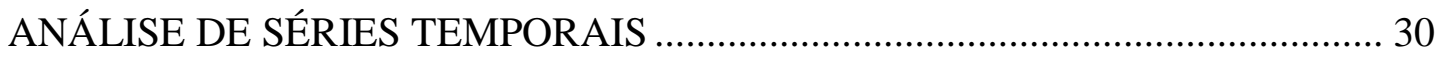

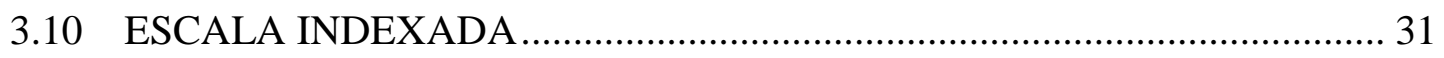

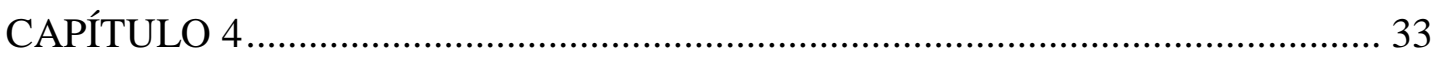

MODELAGEM MATEMÁTICA E RECONSTRUÇÃO DO ESPAÇO DE ESTADOS

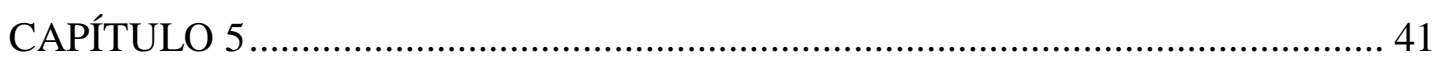

PROCESSO EXPERIMENTAL E ANÁLISE DOS RESULTADOS ......................... 41

5.1 ESCANEAMENTO DO AFM EM MOVIMENTO RETÍLINEO................. 44

5.2 SÉRIES TEMPORAIS CONSTRUIIDAS COM DEFLEXÕES EM UM ÚNICO PONTO DA AMOSTRA ................................................................................ 50

5.2.1 HISTÓRICO NO TEMPO PARA O MODO TAPPING ……………………... 55

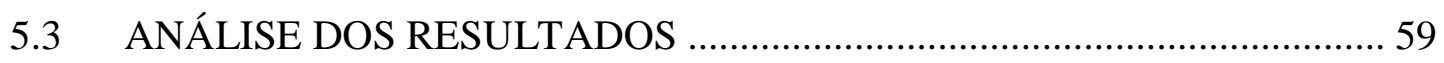

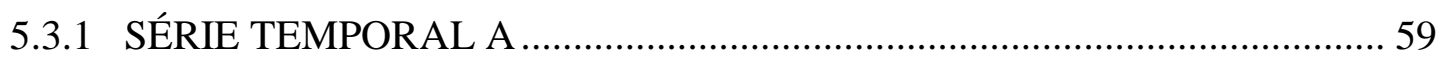

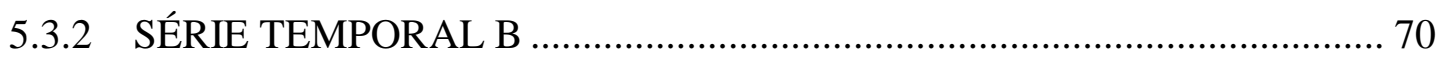

5.3.3 SÉRIE TEMPORAL C ………………………………........................... 79 


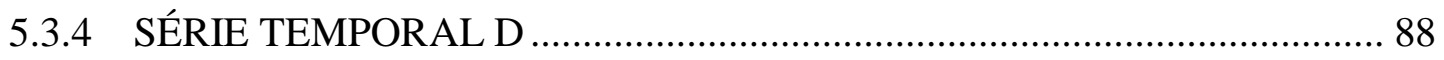

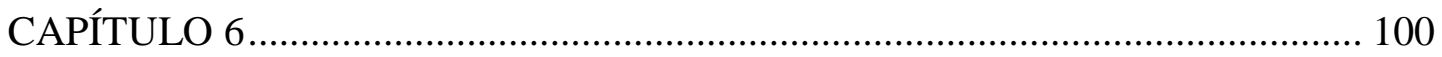

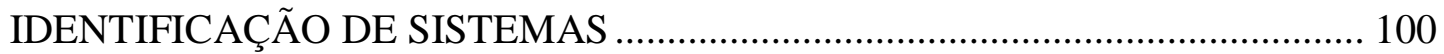

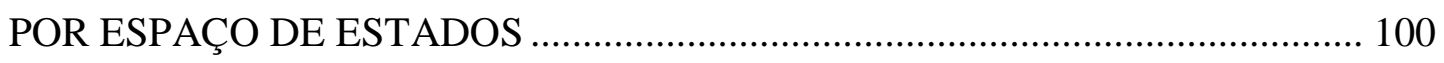

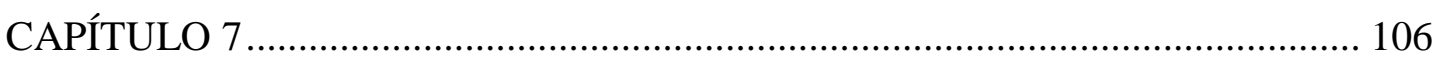

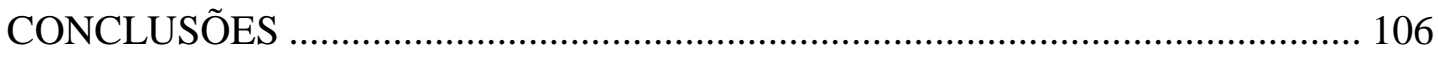

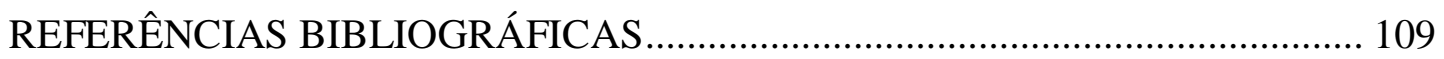

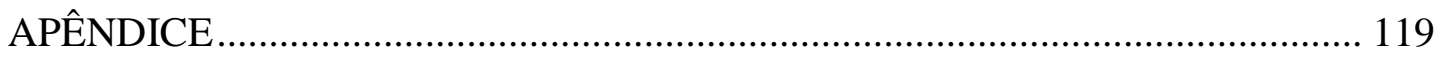

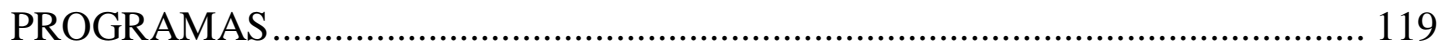

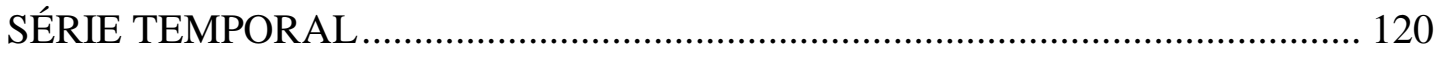




\section{CAPÍTULO 1}

\section{INTRODUÇÃO}

O mundo atual exigiu o avanço da tecnologia de ponta com seus nano-chips e consequentemente houve a necessidade de se criar diversas técnicas para a observação de detalhes ampliados de superfícies para a produção de peças industriais, e assim, exigiu que desde criação das lentes, usadas em microscópios ópticos, inventadas no século XVIII, essencial para o desenvolvimento da medicina, engenharia, materiais, eletrônicos, tecidos, chips, e pesquisas em geral, fossem cada vez mais desenvolvidas.

Observa-se que computadores estão cada vez menores e mais rápidos, assim como nano substâncias que são capazes de desobstruir vasos sanguíneos. Os chips eletrônicos foram se tornando micros e hoje em dia são da ordem de nanômetro, por isso há a necessidade de se buscar avanços em microscópios de força atômica, que conseguem ampliar em muitas milhões de vezes.

Há diversas máquinas que ampliam imagens em pequena escala, e neste século sendo desenvolvidos em sequência dos microscópios baseados em feixes de íons ou de elétrons e são conhecidos como microscópios de força atômica, embora a ideia principal desses microscópios é uma sonda, o qual se difere totalmente dos demais microscópios.

O 'Scanning Tunelling Microscope' foi inventado por Gerd Binnig e Heinrich Rohrer, da IBM de Zurich, em 1981 e foi o primeiro instrumento capaz de gerar imagens reais de superfícies com resolução atômica. Em 1986 os inventores ganharam por ele, o Prêmio Nobel de Física. A partir de uma modificação do microscópio de tunelamento, combinado com um aparelho para medir rugosidade em escala microscópica, Binnig, Quate e Gerber, desenvolveram o microscópio de força atômica em 1986.

A microviga do AFM é um dos principais responsáveis pelo processo de ampliação de imagem e por isso existem muitas pesquisas sobre o assunto. Modelagens da dinâmica comportamental da microviga do AFM é muito estudada pois ela apresenta movimentos sem padrões previsíveis de serem observadas e por isso pode-se aplicar diversas técnicas de estudos de caos e semi-caos. 
Basicamente o AFM é composto por uma microviga com uma ponta que varre a superfície da amostra em estudo. É aplicada uma excitação harmônica que se une a forças de interação entre os átomos da ponta e os da superfície a ser ampliada. A microviga vibra muitos milhões de vezes por minuto e utilizando recursos computacionais, os resultados das alturas da superfície são transformados em imagens da amostra através de rápidos cálculos estatísticos das vibrações da microviga.

Assim as forças de interação são de vários tipos, mas fundamentalmente resumem-se a forças atrativas de "Van der Waals", de origem química, que agem a distâncias entre 100 e algumas unidades de nanômetros (1 nanômetro) e, forças repulsivas que provêm do princípio de exclusão de "Pauli" e, que agem quando a ponta aproxima-se muito da superfície e, os átomos da sonda e da amostra estão tão próximos que começam a se repelir.

As forças de interação entre a ponta da microviga (alguns autores ou pesquisadores chamam na de microviga ou sonda) aparecem entre a ponta e os átomos da superfície da amostra analisada e provocam a deflexão da microviga (microviga). Esta interação resultante pode ser atrativa ou repulsiva. Quando há uma mudança na topografia da superfície (aclives, declives, decorrentes de rugosidades) temos uma mudança na deflexão do microviga. As grandes distâncias entre a ponta e a superfície, a interação é predominantemente atrativa, devida principalmente à força de Van der Waals. A microviga ao se aproximar da ponta da superfície, os orbitais eletrônicos dos átomos da ponta e da superfície ficam próximas e começam a se repelir. Esse processo de atrair e repelir a microviga faz com que a dinâmica dela seja altamente não linear ou caótica. O AFM oferece a possibilidade de obter imagens de resolução atômica com um mínimo de preparação prévia das amostras. As forças de interação acontecem entre a ponta e os átomos da superfície da amostra analisada. As forças de atração são da ordem de $10^{-9} \mathrm{~N}$ e provocam uma deflexão da microviga onde a ponta está montada, fazendo variar a posição de um feixe laser sobre um quadro fotodiodo, gerando sinais elétricos que são processados e geram um mapeamento da superfície em escala nanométrica (BOWEN et al., 2009). As forças entre a amostra e a ponteira alteram as amplitudes das deflexões da microviga que geram imagens da superfície da amostra que é detectada por um sensor. O controle de movimentos em distâncias tão pequenas é possível graças ao uso de cerâmicas piezo elétricas. As cerâmicas funcionam como dipolos. Nestes materiais ocorre uma mudança (deformação) em suas dimensões quando é aplicada uma tensão elétrica. São obtidos movimentos em $(\mathrm{x}, \mathrm{y}, \mathrm{z})$ na ordem de alguns nanômetros, o suficiente para que a ponteira possa varrer a superfície sofrendo interações com os átomos do material ( atrativas /repulsivas ) (EISENSCHITZ, E. e LONDON, F., 1930). 
A varredura é feita em linha podendo ser realizada de três modos básicos. No modo contato, intermitente ou não contato dependendo da possível topografia da amostra e dos objetivos de analise.

A hipótese delimitadora da presente pesquisa consiste em apresentar e formalizar o estudo da dinâmica da microviga do microscópio de força atômica. Basicamente a vibração da microviga é a principal parte do processo de ampliação de imagem, já que é ela quem faz a topografia da amostra ampliada e por isso há a necessidade de se analisar sua dinâmica que na maioria das vezes se apresenta caótica. Para isso uma comparação com um modelo matemático e o trabalho com as séries temporais que registram as deflexões são feitas por dois métodos. Há uma abordagem que se faz pela identificação de sistemas e outra pela reconstrução do espaço de estados. A seguir são apresentados os objetivos desse trabalho.

\subsection{OBJETIVOS DO TRABALHO}

O objetivo deste trabalho é o de identificar situações caóticas através da dinâmica da microviga do AFM investigada por meio de séries temporais obtidas de experimentos. Os objetivos específicos da presente tese são os seguintes:

1) Obter uma série temporal de uma microviga de um microscópio de força atômica em modo não contato e em modo 'soft tapping' em diversas situações através de experimentos em um microscópio de força atômica; analisando uma superfície de borracha, um filme, e safira, em meio líquido, nitrogênio ou oxigênio, com diferentes tipos de ponteira que variam o coeficiente elástico da microviga.

2) Propor um modelo matemático para a dinâmica da microviga AFM em modo não contato. $\mathrm{O}$ modelo considera a massa da microviga, a rigidez do sistema, a interação ponta-amostra e a geometria da ponta da microviga.

3) Reconstruir o espaço de estados a partir da série temporal, buscando um espaço de fase baseado na teoria de Kennel (1992) onde é usada a teoria da informação mútua e a teoria dos falsos vizinhos. 
4) Aplicar a técnica de identificação de sistemas no modelo de AFM por espaço de estados. O modelo identificado será comparado com modelo matemático identificado com a série temporal para se verificar a validade do modelo.

5) Analisar cada série temporal, obtida experimentalmente, em função da altura em que a microviga está fixada, gerando um mapa para localizar as regiões com maior ou menor incidência de movimentos caóticos.

\subsection{APRESENTAÇÃO DOS CAPÍTULOS}

Esta tese de doutorado é desenvolvida em sete capítulos, que abrange os seguintes conteúdos:

No capítulo 1 é introduzido um pouco da história do desenvolvimento da microscopia de força atômica e apresenta o objetivo da tese. Nesse capítulo é mostrado também a forma organizacional da tese, expondo as ideias de como será desenvolvido o tema a ser estudado.

No capítulo 2 é apresentada uma breve visão das propriedades e funcionamento do microscópio de força atômica. É feita a revisão bibliográfica com diversos trabalhos e um histórico do AFM. O modo de funcionamento, contato, não contato e contato intermitente, são descritos neste capítulo. É apresentada também uma revisão de alguns modelos matemáticos constitutivos dos microscópios de força atômica.

No capitulo 3 é realizada a contextualização teórica. As séries temporais são descritas com conceitos de validação e predição. Nesse capítulo são apresentadas técnicas de reconstrução do espaço de estados e do método de identificação de sistemas por espaço de estados.

No capítulo 4 é proposto o modelo que é adotado para a formulação das equações matemáticas. Aplica-se o método de reconstrução do espaço de estados para se comparar os retratos de fases das séries temporais. 
No capítulo 5 é descrito o processo experimental. Mostram-se também como as séries temporais de um microscópio de força atômica reagem ao serem captadas vibrando paradas em um único ponto e vibrando por um caminho. Por fim, apresenta-se uma análise do comportamento caótico das séries em diversas situações de escaneamento através do teste 0-1, escala indexada e reconstrução do espaço de estados.

No capítulo 6 é apresentada a identificação de sistemas para a série temporal obtida através do método linear de espaço de estados.

No capítulo 7 apresentam-se as conclusões finais da presente tese. Após as conclusões, a tese contém um apêndice com programas computacionais e um exemplo de uma série temporal. 


\section{CAPÍTULO 2 \\ REVISÃO BIBLIOGRÁFICA DESCRIÇÃO DO PROBLEMA AFM}

Neste capítulo será apresentada a revisão bibliográfica de sistemas de microscopia de força atômica, através da descrição dos seguintes tópicos: problema AFM; força de Van der Waals; modo de obtenção de imagens no AFM e modelos do oscilador AFM.

As figuras 2.1 e 2.2 ilustram o funcionamento do AFM e a ação da microviga. As mudanças na saída do foto-detector, também são utilizadas para a realimentação, isto é, para ajustar uma cerâmica piezelétrica, na direção vertical z, cujo valor é registrado em função das coordenadas $(\mathrm{x}, \mathrm{y})$, para depois ser traduzido em uma função topográfica $\mathrm{z}(\mathrm{x}, \mathrm{y})$. As coordenadas $(\mathrm{x}, \mathrm{y})$, também são monitoradas através, de tensões aplicadas às cerâmicas piezelétricas colocadas nos "drives" $(\mathrm{x}, \mathrm{y})$ do "scanner", assim como na altura $\mathrm{z}$. (EMBRAPA; 1999). Essas coordenadas x são valores que são afetadas diretamente por forças que agem entre a sonda e a amostra. As diferentes técnicas fornecem diversas oportunidades para mostrar imagens de diferentes tipos de amostras e para gerar uma ampla gama de informações. O controle de movimentos em distâncias tão pequenas é possível graças ao uso dessas cerâmicas piezelétricas. Estes materiais comportam-se de forma, tal que ocorre uma mudança em suas dimensões quando neles é aplicada uma tensão elétrica. $\mathrm{O}$ material PZT é um dipolo que se deforma fisicamente quando há uma ddp entre seus terminais. Por outro lado, se deformada, apresenta uma ddp, gerada por um campo elétrico com um eixo transversal àquele onde foi aplicada a pressão. 


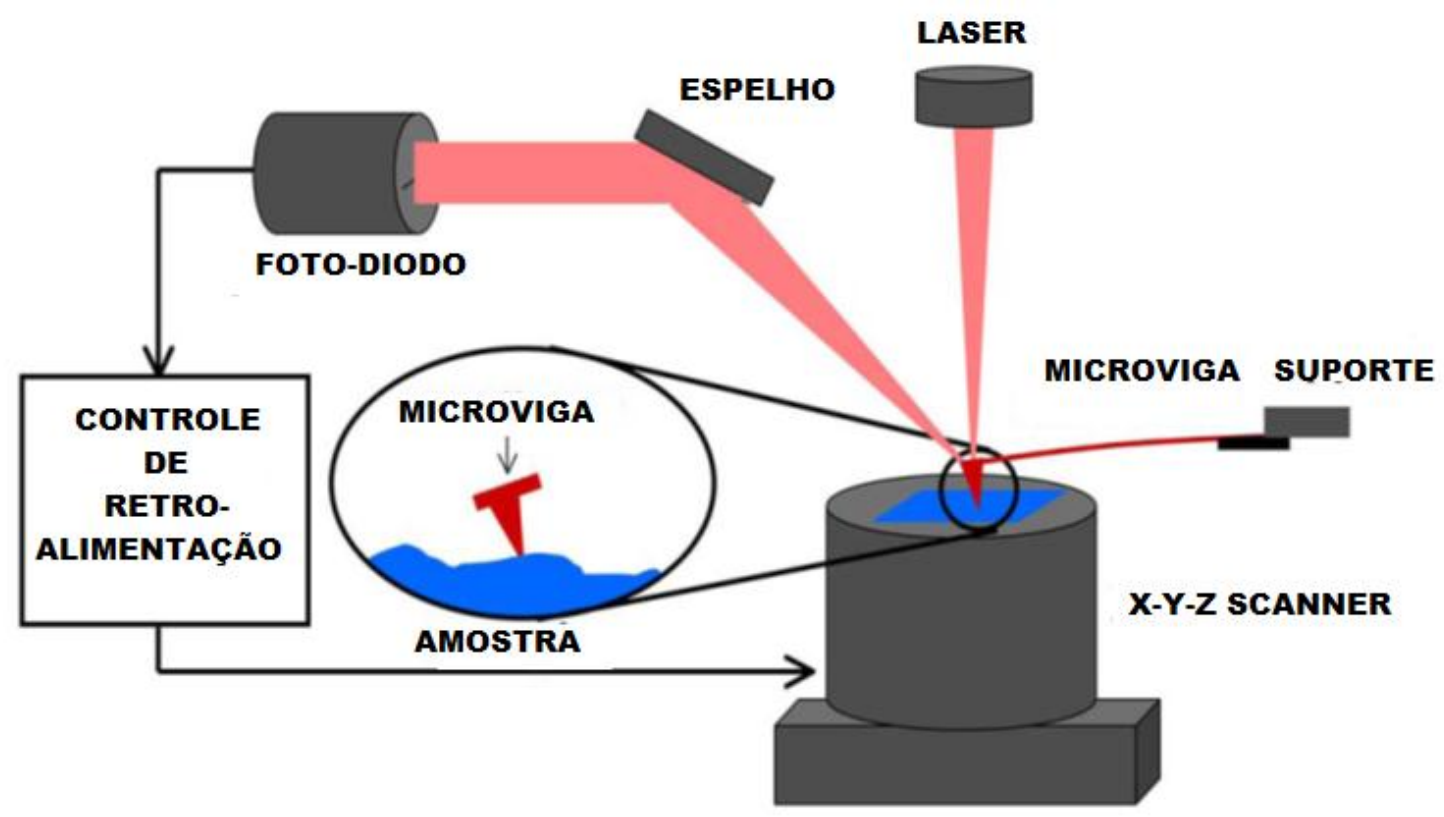

Figura 2. 1 - Esquema de funcionamento do AFM adaptado de (SEBASTIAN et Al., 2007)

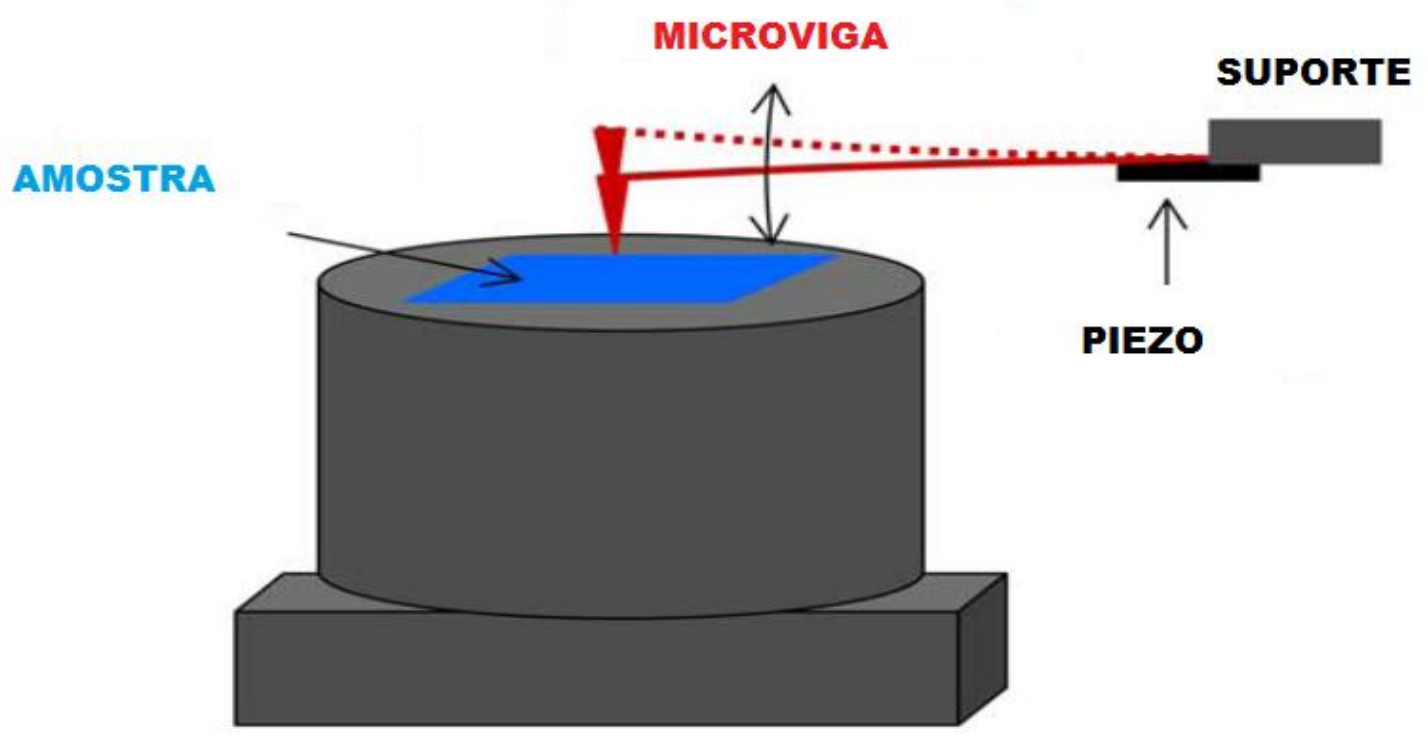

Figura 2. 2 - Esquema da ação do "micromicroviga" durante a operação em "AFM" Adaptado de (SEBASTIAN et Al., 2007)

Os métodos de produção de imagens, também chamado de digitalização ou modos de operação, referem-se principalmente a distância mantida entre a sonda e a amostra no momento da digitalização, e as formas para mover a ponta sobre a superfície (ZANNETE, 1997). Há muitas formas possíveis de se fazer imagens, devido às interações diferentes, dependendo da distância entre a ponta e a amostra, bem como o regime de detecção 
utilizado. A escolha do modo apropriado depende da aplicação específica do que se quer fazer. Quando a ponta se aproxima da primeira amostra é atraído para a superfície, devido à existência de forças atrativas da região, como as forças de Van der Waals (ZANNETE, 1997). Esta atração aumenta até a ponta ficar muito próxima da amostra e os átomos de ambas se aproximam de modo que seus orbitais eletrônicos começam a repelir. Esta repulsão eletrostática enfraquece a força atrativa à medida que aumenta a distância. A força é nula quando a distância entre os átomos é de cerca de alguns Angstroms (aproximadamente a distância característica de uma ligação química). Quando as forças se tornam positivas, podemos dizer que os átomos da ponta e da amostra estão em contato e as forças repulsivas dominam (EISENSCHITZ, E. e LONDON F., 1930).

Em 1873 Van der Waals postulou a existência das forças intermoleculares, que ficaram conhecidas pelo seu nome. Fritz London descreveu a natureza delas e as atribuiu ao movimento dos elétrons dentro das moléculas. As interações das forças de Van der Waals devidas especificamente às flutuações dos elétrons em moléculas ficaram conhecidas com o nome de London, de flutuação de cargas, eletrodinâmicas, ou de dispersão e estão presentes até em moléculas polares permanentes. E por isso as Forças de Van der Waals têm um papel muito importante em fenômenos diversos tais como adesão, tensão superficial e muitos outros. (LONDON F., 1930).

As forças de dispersão geralmente são maiores que as de orientação e de indução, exceto no caso de moléculas polares muito fortes como as de água. As forças de dispersão ou, em geral, as forças de van der Waals são normalmente atrativas e crescem rapidamente quando as moléculas, átomos ou corpos se aproximam uns dos outros (Distancia ponta a amostra) (ISRAEACHVILI, 2005). A seguir são descritos os modos de se fazer imagens no AFM.

O AFM pode ser operado de diversos modos, os quais fornecem grande variedade de opções para a construção de imagens de diferentes tipos de amostras, gerando diferentes tipos de informação. Os modos de se fazer imagens podem ser classificados em dois tipos operação em contato ou em não-contato - dependendo das forças líquidas entre a ponteira e a amostra.

Quando o aparelho é operado na região atrativa, o método chama-se não-contato. Nesta região, a microviga do AFM se enverga na direção da amostra. A operação na região repulsiva chama-se contato e a microviga se dobra, afastando-se da amostra. No modo de não-contato ela é atraída pelas forças de capilaridade da camada de contaminação ou pelas forças de Van der Waals, quando a amostra é limpa. No modo de contato, a deflexão da 
microviga é na direção oposta à da amostra . A força é repulsiva e a microviga (microviga), em geral em $\mathrm{V}$, é mais flexível, para conseguir uma boa flexão sem deformar a amostra.

A constante de elasticidade " $\mathrm{K}$ " da mola das microvigas moles mais usadas variam entre 0,02 e $0,05 \mathrm{~N} / \mathrm{m}$. Eventualmente, podem ser utilizadas microvigas mais rígidas. Entretanto, devem ser operadas a altas forças. Neste caso, durante a aproximação da ponteira, a microviga é atraída fortemente para a superfície, podendo distorcê-la ou danificála se a amostra for pouco resistente a pequenos impactos. As constantes de mola das microvigas duras mais utilizadas variam entre 20 e $80 \mathrm{~N} / \mathrm{m}$. (ZANNETE, 1997). Este modo é muito eficiente também para a construção de imagens de amostras submersas ou em meio líquido. O processo é útil para a preservação de amostras mais moles, pois há um certo amortecimento em meio líquido.

Em resumo, se espera que as medidas com AFM no regime de contato explorem fundamentalmente as forças de repulsão íon-íon, que decaem rapidamente com o aumento da separação ponteira-superfície. A forte dependência com a distância das forças repulsivas íon-íon, fornece a chave para a grande resolução espacial atingida pela microscopia de força de contato.

No modo contato, a haste sobre a qual está presa a ponta, varre nas direções planas x e y, e na altura z. A varredura é feita por uma cerâmica piezo-elétrica presa à haste ou à amostra. A interação ponta - amostra é monitorada por um laser que é incidido sobre a haste e refletido para dois pares de foto-detectores. A deflexão da haste pode ser medida devida a uma diferença de sinal dos fotodetectores (ZANNETE, 1997).

Durante a varredura, através de uma realimentação com o computador, são realizadas correções na direção z para manter a deflexão da haste constante. Deste modo os valores de z são armazenados na memória, para cada posição, formando uma imagem completa da morfologia da superfície.

No modo contato intermitente a haste oscila próximo a sua freqüência de ressonância, o sinal obtido dos fotos-detectores mede a amplitude de oscilação, que deve ser mantida constante. Neste caso, a ponta toca suavemente a superfície da amostra, e elimina a força de atrito presente no modo contato. Esse processo é frequentemente chamado de 'tapping'. Neste caso os toques constantes da esfera na amostra podem danificar a estrutura física da mesma.

No modo não contato, quando a ponteira se aproxima da amostra, é primeiramente atraída pela superfície, devido a uma ampla gama de forças atrativas existentes na região, como as forças de Van der Waals. Esta atração aumenta até que a ponteira aproxima-se 
muito da amostra, os átomos de ambas estão tão próximos que seus orbitais eletrônicos começam a se repelir. Esta repulsão eletrostática enfraquece a força atrativa à medida que a distância diminui. A força anula-se quando a distância entre os átomos é da ordem de alguns Ângstroms (da ordem da distância característica de uma união química), isso se repete sucessivamente (atração e repulsão), fazendo com que não haja contato.

Assim, os modos de se fazer imagens são divididos em modos não contato, contato e contato intermitente e por isso pode se dividir graficamente na figura 2.3, quando é feito um estudo da força de atração em relação a distância da microviga até a amostra.

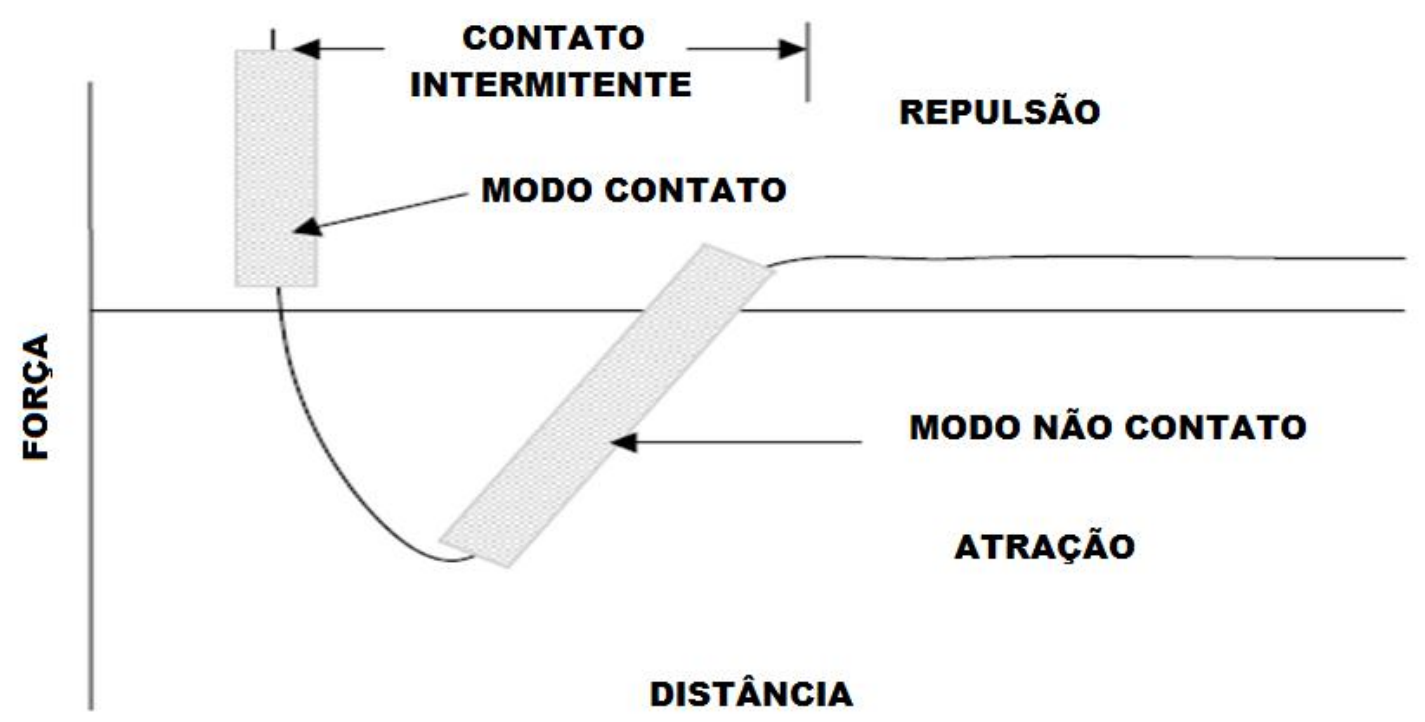

Figura 2. 3 - Os regimes de força do AFM e os três modos de operar. Adaptado de Bowen (2009).

O Modo contato de operação está no regime de força de repulsão, em que a sonda é pressionada contra a superfície da amostra, causando uma deflexão para cima da microviga. O modo não-contato repele as forças antes do contato real com a superfície. Com o contato intermitente, a sonda oscila perto da superfície e vem repetidamente para dentro e fora da superfície. A seguir é feita uma descrição de modelos usuais para oscilador do AFM.

A dinâmica não-linear de microscopia de força atômica (AFM) é um tema emergente de pesquisa em Ciências e Engenharia, desde a sua descoberta por Binning et al. (1986) e de acordo com uma série de autores como Jalili et al. (2004), Wang (2009), Garcia et al. (2000), os modelos matemáticos que regem a dinâmica das microvigas dos AFM, geralmente, têm uma das duas seguintes simplificações: supondo que a microviga curva-se quando uma carga estática é aplicada na ponta dessa microviga (ponteira) e com a rigidez 
estática correspondente para obter um único grau de liberdade do modelo massa-mola ou então um modelo de discretização da equação clássica da viga com base em seu modo normal de vibrar conduzindo a graus de um ou vários graus de liberdade.

De acordo com Jalili et al. (2004) um modelo matemático para uma interação amostra microviga de um processo AFM pode ser representado por um modelo massa- mola, com uma constante $k$ da mola e de massa equivalente m. A microviga interage com a amostra, através de uma ponta, que é montada no fim da microviga. O sistema ponta da microviga (ponteira) até a amostra é considerada com uma esfera de raio $\mathrm{R}$ e massa $\mathrm{m}_{\mathrm{e}}$, que está suspenso por uma mola de rigidez $\mathrm{k}$. As forças de Van der Waals denotam a atração / repulsão em vigor. Isto é, as forças de interação entre a ponta do microviga e a superfície da amostra. No modelo de Jalili et al. existe uma coordenada para representar a distância entre a estrutura de base fixa e a amostra. Os resultados obtidos por Jalili et al. estão em completo acordo com os experimentos, publicados na literatura atual. (ver WANG, PAI E YAU (2009), ASHAB, SALAPAKA, DAHLEH E MEZIC (1987), JALILI E LAXMINARAYANA (2004)).

Outra parte do AFM é a ponteira que é apoiada na extremidade da microviga e que pode ter forma de $\mathrm{V}$ ou de haste, em geral, retangular. A força que a amostra exerce sobre a ponteira é determinada pela deflexão do microviga, dada pela lei de Hooke $\omega=-k x$, sendo $x$ o deslocamento do microviga e $k$ a sua constante de mola própria, determinada pelas características de construção. Além da ponteira a microviga é outro componente do AFM que deflete em altas amplitudes para atingir alta sensibilidade. Portanto, a mola deve ter a constante com valores baixos. Por outro lado, é preciso uma alta freqüência de ressonância para diminuir a sensibilidade a vibrações mecânicas, especialmente quando se está fazendo a varredura. Como a freqüência de ressonância do sistema da mola é dada por $\mathrm{f}$ $=(\mathrm{k} / \mathrm{m})^{1 / 2}$, e a massa $\mathrm{m}$ da microviga é da ordem de nanômetros, mesmo para uma mola flesível (k pequeno >>m) a frequência é alta. Portanto, devem ser utilizadas técnicas especiais de micro fabricação para a produção de microvigas que operam em altas frequências.

As microvigas em forma de $\mathrm{V}$ vistas na figura 2.4 possuem uma dureza lateral grande, se comparadas com as de forma retangular. Isso reduz a sensibilidade a forças laterais de atrito que poderiam resultar em séria degradação das imagens, especialmente de superfícies topográficas com variações grandes em altura e inclinação. 


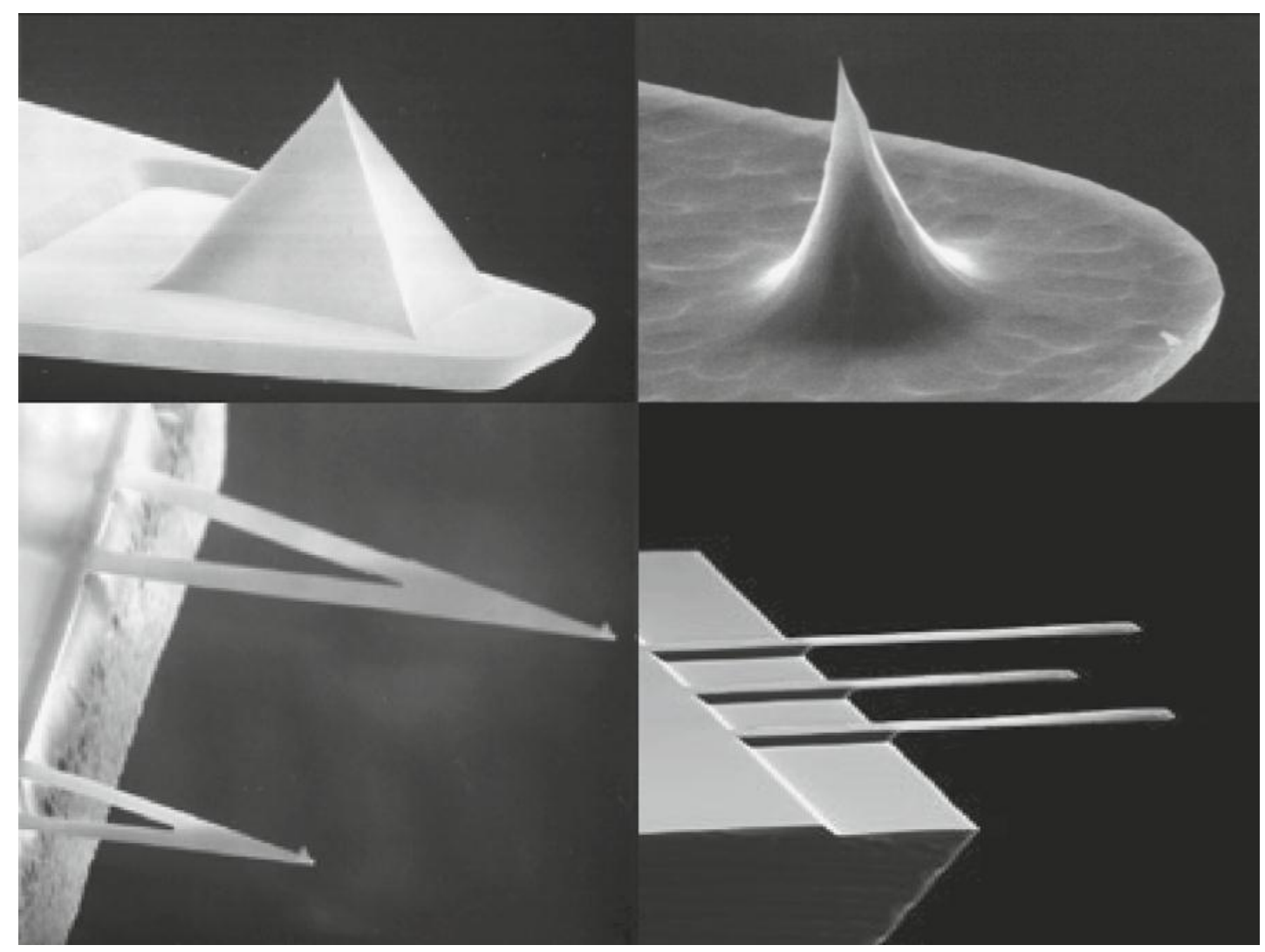

Figura 2. 4 - Tipos de ponteiras. Bowen (2009).

A imagem superior ao lado esquerdo da Figura 2.4 mostra a ponteira piramidal. A imagem superior à direita mostra uma ponteira cônica para alta resolução. A inferior esquerda mostra ponteiras em V para modo contato ou 'soft tapping', e a sua direita mostra ponteiras com diversos tamanhos. (BOWEN et al., 2009).

Alguns dos parâmetros do experimento são visualizados ao se abrir os arquivos dados captados no processo de ampliação de imagem pelo modo 'soft tapping' no programa nanoscope ${ }^{\circledR}$ software 6.13 (BRUKER, 2004).

\begin{tabular}{|c|c|}
\hline Channel data & \\
\hline - Image Data & TM Deflection \\
\hline - XDatt Type & Time \\
\hline - Sens. TM Deflection & $100.0 \mathrm{~nm} / \mathrm{N}$ \\
\hline - Plot Units & Metric \\
\hline - Spring Constant & $42.00 \mathrm{~N} / \mathrm{m}$ \\
\hline - Display Mode & TM Deflection Us, Time \\
\hline
\end{tabular}

Figura 2. 5 - Constante elástica visualizada no programa nanoscope ${ }^{\circledR}$ (Bruker, 2004) 


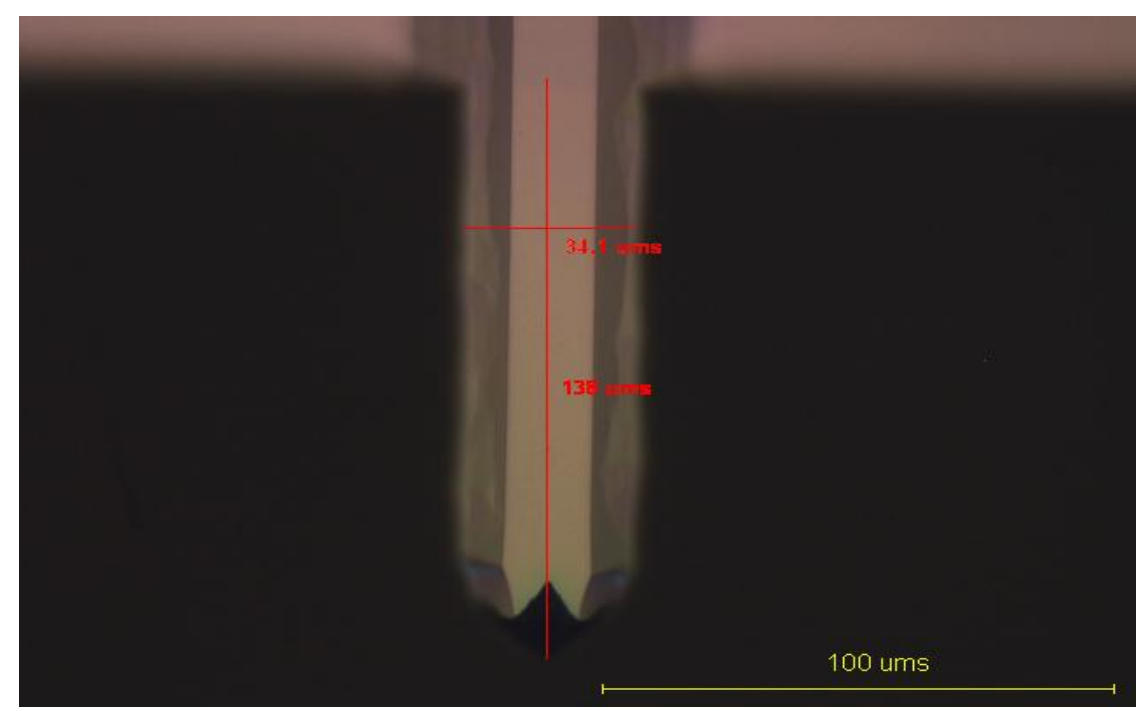

Figura 2. 6 - Dimensões da microviga do AFM

Há extensos tópicos a se explorar sobre os AFMs, desde a modelagem e análise da dinâmica da microviga através de modos estatísticos, explorando séries temporais colhidas em experimentos, modelagem pela Mecânica Clássica ou por elementos finitos. Alguns modelos são diferenciados pelo tipo de material a se fazer a varredura e outros fazem uma análise do material em meio líquido. Muitas equações modelam o AFM em modo não contato e em modo Tapping. Os modelos apresentados a seguir são os de Jalili, Dadfarnia, e Dawson (2004), Wang et al. (2009) e Nozaki et al. (2012).

O modelo apresentado por Jalili, Dadfarnia, e Dawson (2004) relata o microscópio de força atômica em modo não contato e a faz uma análise da dinâmica da microviga do AFM. Técnicas de imagem atual AFM são muitas vezes baseadas em um modelo discreto, com parâmetros e equações diferenciais ordinárias que modelam o funcionamento das microvigas juntamente com um método para a estimativa de interação atômica (especialmente no modo sem contato).

Uma vez que a magnitude da força de interação está dentro da faixa de nano-Newtons, a estimativa da força atômica é crucial para a imagem precisas. $\mathrm{O}$ trabalho de Jalili et al. (2004) visa melhorar a modelagem da microviga de um AFM através do desenvolvimento de uma distribuição geral dos parâmetros. Eles se aproximam da modelagem base que revela um maior conhecimento sobre as características fundamentais da interação da amostra à microviga. 


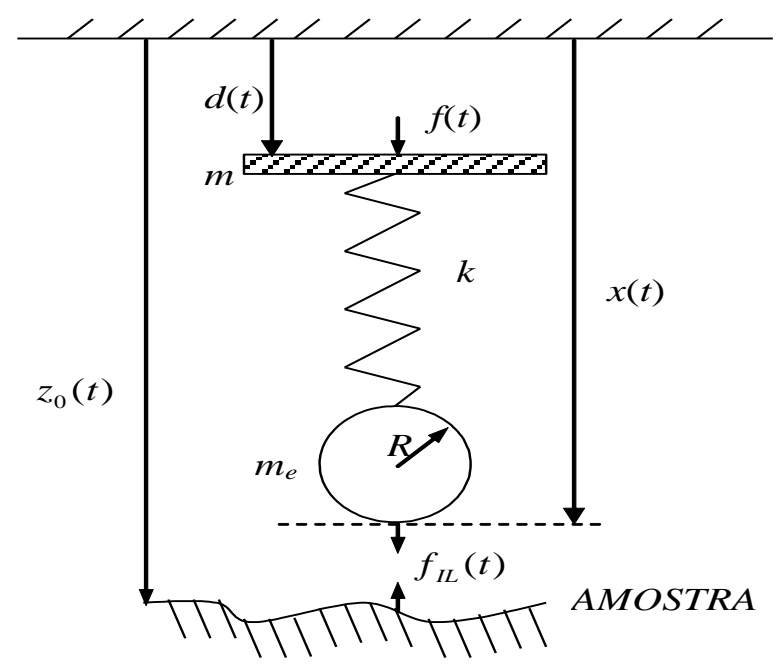

Figura 2. 7 - Modelo proposto por Jalili et al. (2004).

O modelo de Jalili et al. (2004) é representado basicamente por $F=m \ddot{x}+c \dot{x}+k x$ onde observa-se um modelo de equação diferencial massa mola com $\mathrm{F}$ sendo a força de Van de Waals mais a força de excitação harmônica. A Fig. 2.7. mostra as principais variáveis que influenciam na vibração da microviga. A força de Van der Waals é quem atrai a microviga e quando está muito próxima à amostra, ela é repelida. O parâmetro $m$ é a massa da microviga, $R$ é o raio do tip que fica na ponta da microviga. O $m_{e}$ é a massa da ponta do tip, $z_{0}(t)$ é a distância entre altura do suporte da microviga até a amostra, $k$ é a constante elástica da microviga e $f(t)$ é a excitação harmônica de forma senoidal aplicada no suporte da microviga. A variável $x(t)$ são deflexões da microviga a uma altura $d(t)$ que serão captadas como série temporal no capítulo 4.

A figura 2.8 ilustra um retrato de fase comum para osciladores de AFM. Esse retrato de fase extraído de Wang (2008) mostra uma possível batida do lado esquerdo, apesar de ela representar um modelo não contato, onde a microviga não encosta na amostra. Os autores usaram um modelo massa mola com acoplamento da força de Van der Waals. 


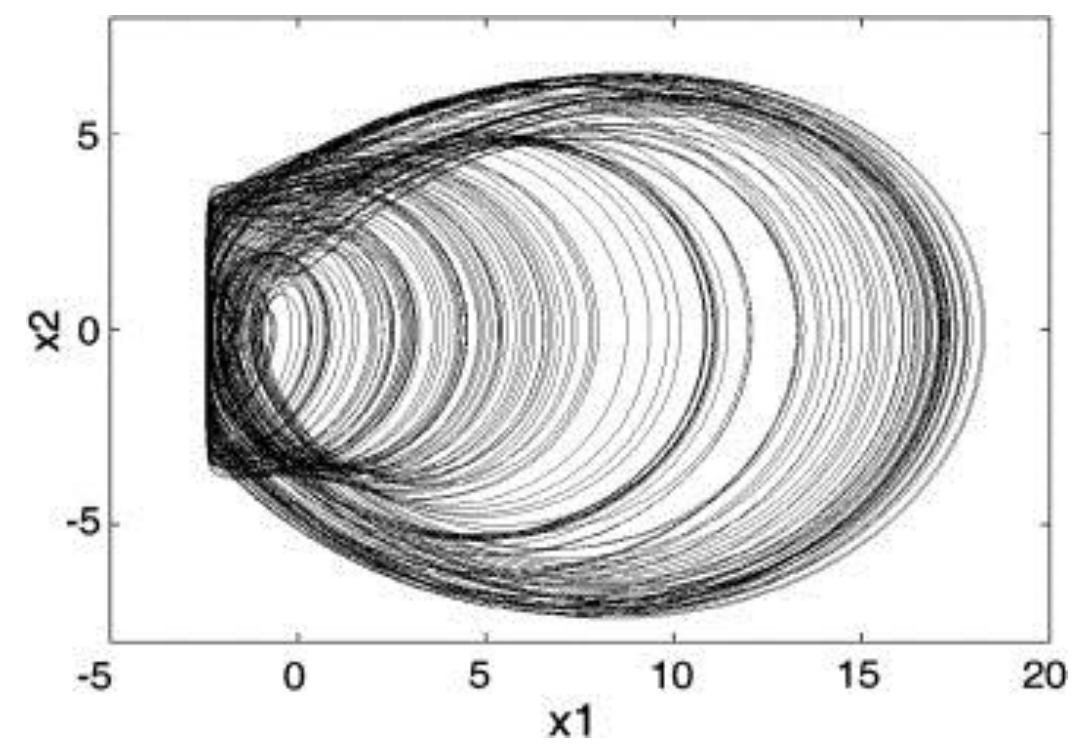

Figura 2. 8 - Retrato de fase de Wang et al. (2008)

Vários estudos têm sido desenvolvidos sobre a dinâmica do AFM. Vários autores têm estudado mecânica do microscópio de força atômica com base em suas dinâmica não-linear, a modelagem através do Hamiltoniano (WANG et al., 2009), observando-se o caos através expoente de Lyapunov, bacias de atração (BALTHAZAR et al., 2012), técnicas de bifurcações e tomada de controles otimizados para AFM sem contato (NOZAKI et al., 2012), no modo contato intermitente (RODRIGUES et al., 2014), (BALTHAZAR et al., 2013), (BALTHAZAR et al., 2012), estudando no modo contínuo (CLAEYSSEN, et al., 2012), o desenvolvimento do projeto 'Phase locked loop' para melhorar o sistema de controle da frequência modulada de microscópio de força atômica (FM-AFM) com sistema de controle não-linear encontra-se em (BUENO et al., 2011), Balthazar et al. (2014) apresenta um controle para suprimir o movimento caótico da microviga, através de duas técnicas: o controle ótimo linear feedback (OLFC) e o State-Dependent Riccati Equation (SDRE). Além disso, a robustez de ambas as técnicas de controle é testada considerando incertezas de parâmetros no modelo FM-AFM e no sinal de controle (BALTHAZAR et Al., 2011). Em operação sem contato, a microviga posiciona-se a uma distância de alcance longo da superfície da amostra e, neste caso, as forças de interação ocorrem mais distantes da superfície da amostra (forças de Van der Waals são predominantes). Em técnicas de nãocontato dinâmicos, tais como o alcance de força atômica de micro frequência modulada (FM-AFM) ou o microscópio de força atômica de modulação em amplitude (AM-AFM), a microviga vibra a uma frequência e amplitude fixa. Neste caso, as forças de interação TIPamostra são modulados no movimento da microviga e o sinal de controle é muitas vezes usado para gerar imagens topográficos da superfície da amostra. Diferentemente da técnica 
de contato simples, técnicas sem contato alcançam verdadeira resolução atômica em uma operação estável (BUENO et al., 2011, 2012.); (BALTHAZAR et al.,2012). Os sistemas de AFM podem sofrer comportamentos indesejáveis e inesperados, como instabilidade e movimento caótico, devido aos efeitos de não-linearidades do sistema. Como resultado, as imagens de AFM obtidas com a microviga em movimentos caóticos, mostram erros de metrologia significativas em nanoescala (HU e RAMAN, 2006). Um número de métodos de controle com o objetivo de atenuar ou suprimir os efeitos das não-linearidades têm sido estudados por Hornstein et al. (2008) e Yabuno (1999). No capítulo 3 apresenta-se a contextualização teórica com descrição das séries temporais, métodos de informação mútua e conceitos do teste 0-1 e escala indexada. 


\section{CAPÍTULO 3 \\ CONTEXTUALIZAÇÃO TEÓRICA}

\subsection{SÉRIES TEMPORAIS}

Cada vez mais o estudo de séries temporais vem ganhando espaço nas pesquisas científicas, no cotidiano e outros setores. Há uma série de trabalhos utilizando séries temporais e especificamente na microscopia de força atômica é um trabalho inovador.

Segundo Morettin e Toloi (2006), uma série temporal é qualquer conjunto de valores observáveis e ordenadas no tempo. Ehlers (2009) mostra que a característica mais importante deste tipo de dados é a vizinhança dependente, e a análise e a modelagem desta dependência são fundamentais no entendimento das séries temporais. Enquanto em modelos de regressão, por exemplo, a ordem das observações é irrelevante para a análise, em séries temporais a ordem dos dados é definitivamente muito importante. Existem muitos pacotes de programas e toolbox implementados para as séries temporais tal como o pacote Tisean® que é um projeto de software para a análise de séries temporais com métodos baseados na teoria de sistemas não-lineares determinísticos dinâmicos, ou a teoria do caos. O Tisean foi desenvolvido a partir do trabalho de R. Hegger, H. Kantz, e T. Schreiber (2007). Alguns exemplos de séries temporais são: os valores diários do trânsito da cidade de São Paulo; os valores diários de temperatura na cidade de São Carlos-SP; os preços diários de índices da BOVESPA (Bolsa de Valores de São Paulo); os registros de vibrações na asa de um avião em um determinado tempo e condições climáticas; os registros de vibrações de uma sonda de um microscópio de força atômica; os batimentos cardíacos diários; o som da voz humana no processo de fala; a dinâmica de um pêndulo; entre outros.

A análise de uma série temporal com uma abordagem não-linear é um problema que exige certos cuidados, pois deve se trabalhar com técnicas de probabilidade juntamente com um problema topológico. Deve estudar se as séries estão em uma mesma dimensão ou em duas ou mais dimensões.

Respostas simples têm sido repetidamente oferecidas na literatura, citando valores numéricos para as dimensões e os atratores para qualquer sistema. Uma série temporal é dita estacionária quando ela se desenvolve no tempo aleatoriamente ao redor de uma média constante, refletindo alguma forma de equilíbrio estável. Na prática, a maioria das séries apresentam algum tipo de não estacionariedade. As séries temporais são apresentadas em 
forma de função do tempo. A figura 3.1 representa uma série temporal estacionária. A figura 3.2 representa uma série temporal não estacionária.

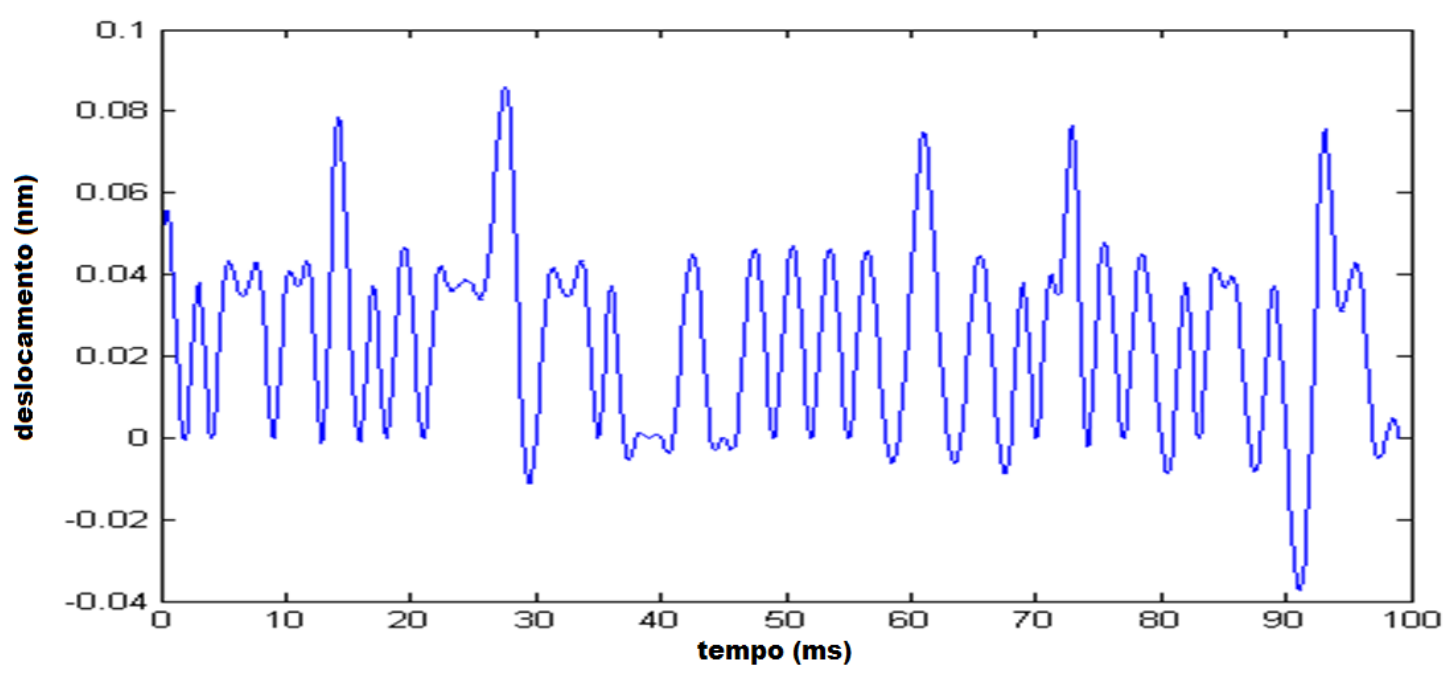

Figura 3. 1 - Exemplo de série temporal estacionária de um AFM (NOZAKI, 2016)

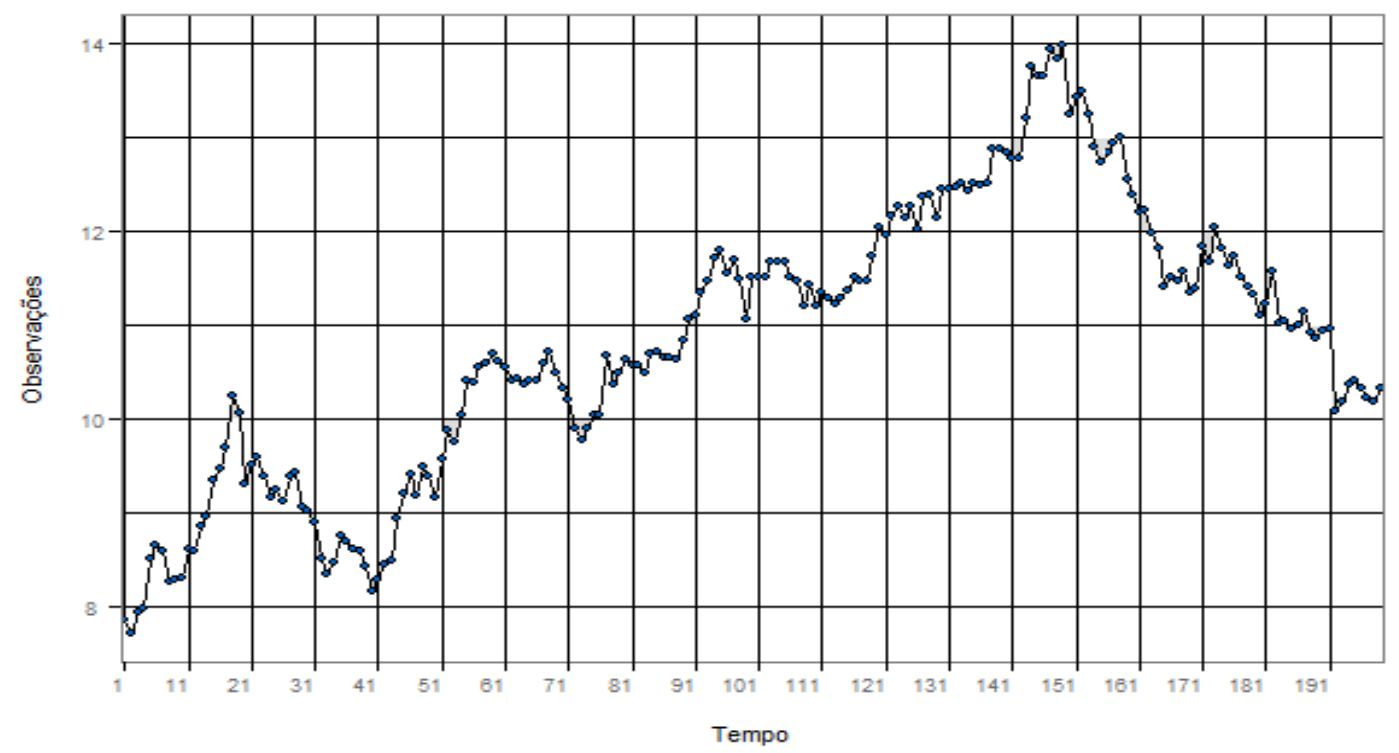

Figura 3. 2 - Série temporal não estacionária de www.portalaction.com.br

Uma série temporal é dita estacionária quando ela se desenvolve no tempo aleatoriamente ao redor de uma média constante, refletindo alguma forma de equilíbrio estável. Na prática, a maioria das séries que encontramos apresentam algum tipo de não estacionariedade, por exemplo, tendência. 


\subsection{RECONSTRUÇÃO DO ESPAÇO DE ESTADOS}

Há modelos matemáticos estabelecidos que detalham a dinâmica da microviga do microscópio de força atômica. A utilização de técnicas de análise de séries temporais para compreender esses modelos é uma alternativa bastante interessante e juntamente com a identificação de sistemas é possível uma análise mais detalhada dos parâmetros e variações que podem afetar o desempenho do escaneamento da imagem. Assim as séries temporais são usadas como variáveis representativas da dinâmica do sistema. Uma vez que o ruído é inevitável em séries temporais experimentais, as técnicas empregadas devem ser robustas a presença de ruídos. Existem diversos métodos utilizados na reconstrução do espaço de estado. O Método das Coordenadas Defasadas (TAKENS, 1981) é utilizado para a reconstrução do espaço de estado do sistema e os parâmetros de defasagem são avaliados pelo Método da Informação Mútua Média e pelo Método dos Falsos Vizinhos Próximos. Esses dois métodos são utilizados em simulações e estão implementados no software TISEAN (HEGGER et. al., 1999).

Packard et al. (1980) propuseram outro método de reconstrução de espaço de estados chamado "método das derivadas" em que as coordenadas são aproximações numéricas das derivadas de ordem sucessivamente superiores de uma variável medida e foi definida por Savi (2006) como:

$$
\dot{S}(t) \approx \frac{S\left[t_{0}+(n+1) \Delta t\right]-S\left(t_{0}+n \Delta t\right)}{\Delta t}
$$

onde $S\left(t_{0}+n \Delta t\right)$ é o sinal, $\mathrm{n}=1,2, \ldots, \mathrm{N}$ é o número total de amostras e $\Delta t$ é o intervalo entre duas amostras. A determinação das outras derivadas utiliza o mesmo procedimento, usando as derivadas de ordem inferior. Uma vantagem do uso das coordenadas derivativas que é o significado físico está diretamente relacionada com as derivadas obtidas. Por outro lado, sua principal desvantagem é a sensibilidade ao ruído.

Segundo Hegger, R., Kantz, H., and Schreiber, T. (1999) a reconstrução do espaço de estado é a técnica de se analisar a série temporal mantendo a dinâmica original do sistema, sob um ponto de vista topológico. Por isso os autores apresentam a reconstrução de espaço de estados pelo método das coordenadas defasadas. Nesse método, a reconstrução do espaço de estado precisa formar um sistema de coordenadas para capturar a estrutura das órbitas no espaço de estado, que pode ser feito usando variáveis defasadas, $S_{n}$, onde $\tau$ é uma 
defasagem no tempo. Assim, isto é possível construir uma coleção de defasagens no tempo para criar um vetor no espaço de dimensão $m$.

Segundo Savi (2006). O método de coordenadas defasadas o espaço de estado do sistema pode ser reconstruído usando a série temporal com as variáveis em atraso, $S(t+\tau)$, onde $\tau$ é o atraso. Então, considerando o sinal do experimento, onde $t=t_{0}+(n-1) \Delta t$ com $n=1,2,3, \ldots, \mathrm{N}$, é possível fazer uma série com atrasos em $m$ dimensões de espaço, $u(t)$, que representa a reconstrução dinâmica do espaço do sistema.

$$
u(t)=\{s(t), s(t+\tau), \ldots, s(t+(m-1) \tau)\}^{T}
$$

Esta abordagem está associada com a determinação dos parâmetros de defasagem: tempo de defasagem, $\tau$, e dimensão de imersão, m. O Método da Informação Mútua Média (Fraser e Swinney, 1986) é uma alternativa para calcular o tempo de defasagem, $\tau$. A determinação da dimensão de imersão, m, por outro lado, pode ser avaliada pelo Método dos Falsos Vizinhos Próximos (Kennel et al., 1992).

\subsection{MÉTODO DA INFORMAÇÃO MÚTUA MÉDIA}

A ideia do método da informação mútua média é criar atrasos aplicados a uma série temporal. A determinação do tempo de defasagem deve ser adequada para obter as variáveis defasadas mais independentes possíveis, utilizando ferramentas de cunho estatístico e probabilístico Fraser e Swinney (1986) estabelecem que o tempo de defasagem $\tau$ corresponde ao primeiro mínimo local da função de informação mútua média $\mathrm{I}(\tau)$, que é definida na equação 3.3 .

$$
I(\tau)=\sum_{n=1}^{N-\tau} \Gamma\left(S_{n}, S_{n+\tau}\right) \log _{2}\left[\frac{\Gamma\left(S_{n}, S_{n+\tau}\right)}{\Gamma\left(S_{n}\right) \Gamma\left(S_{n+\tau}\right)}\right]
$$

onde $\Gamma\left(S_{n}\right)$ é a probabilidade da medida $S_{n}, \Gamma\left(S_{n+\tau}\right)$ é a probabilidade da medida $S_{n+\tau}$, e $\Gamma\left(S_{n}, S_{n+\tau}\right)$ é a probabilidade da medida combinada entre $S_{n}$ e $S_{n+\tau}$. Quando as medidas $S_{n}$ e $S_{n+\tau}$ são completamente independentes o valor de $I(\tau)=0$. Por outro lado, quando $S_{n}$ e $S_{n+\tau}$ são iguais, $I(\tau)$ assume o valor máximo. Portanto, a análise da curva $I(\tau)$ permite determinar o melhor tempo de defasagem para ser usado na reconstrução do espaço de estado. 


\subsection{MÉTODO DOS FALSOS VIZINHOS PRÓXIMOS}

Hegger, R., Kantz, H., e Schreiber, T. (1999) retratam os falsos vizinhos como a hipótese ou a probabilidade de assumir que as dinâmicas do espaço fase representadas por um campo vetorial imprecisa. Observa-se na Fig 3.3 extraída de Viola (2011) após um pequeno intervalo de tempo, as dimensões em que um conjunto de vetores estão imersos em uma dimensão, duas dimensões, em três dimensões ou $n$ dimensões. Em uma dimensão, todos os pontos estão aparentemente próximos. Em duas dimensões observa-se que um ponto está afastado do grupo de pontos e, em três dimensões, dois pontos se afastaram, caracterizando-se como falsos vizinhos.

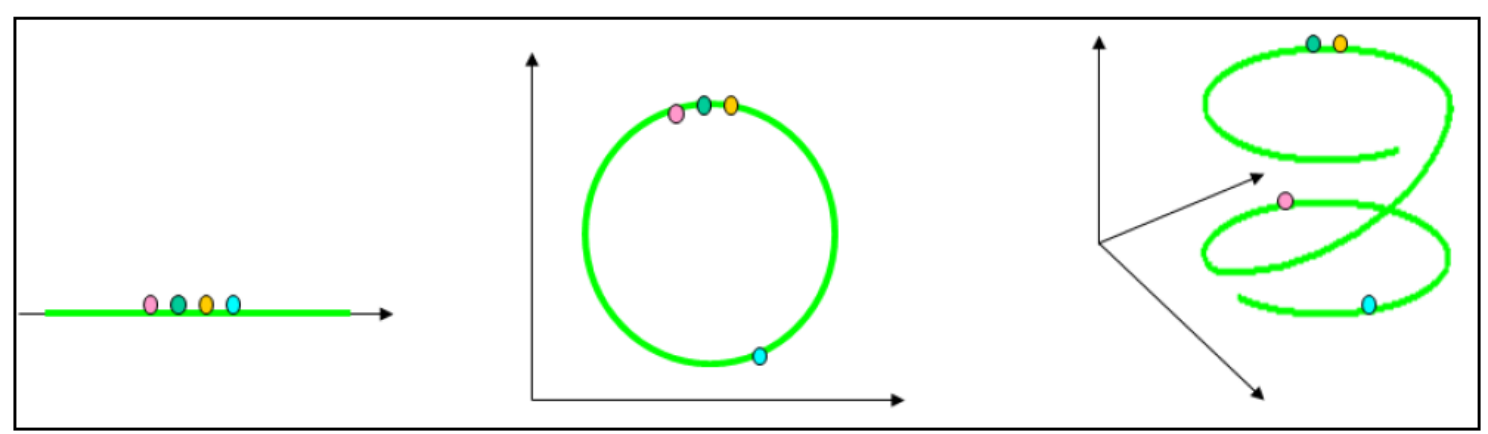

Figura 3. 3 - Figura de Viola (2011) - Possíveis falsos vizinhos próximos e suas dimensões de imersão.

O método dos Falsos Vizinhos Próximos, de Kennel, Brown e Abarbanel (1992), foi originalmente desenvolvido para determinar o número de coordenadas de defasagem no tempo necessárias para recriar a dinâmica de sistemas autônomos, mas isto foi estendido para examinar o problema de determinar a dimensão de imersão adequada. Este método é baseado na busca de uma dimensão mínima onde não ocorra cruzamento de uma órbita consigo mesma. Sendo assim, procura-se determinar se um vizinho é "verdadeiro" ou "falso" considerando a projeção do sistema em uma determinada dimensão.

Em uma dimensão de imersão que seja muito pequena para mostrar o atrator, nem todos os pontos que se encontram próximos um dos outros são vizinhos por causa da dinâmica. Alguns estão realmente longe uns dos outros e simplesmente aparecem como vizinhos por causa da estrutura geométrica do atrator que foi projetado em uma dimensão menor (KENNEL et al., 1992).

Para utilizar o Método dos Falsos Vizinhos Próximos, um espaço de dimensão m é considerado onde o ponto tem r-ésimos vizinhos próximos, $S_{n}^{(\mathrm{r})}$. A condição para decidir se 
os vizinhos são falsos é dada através de uma norma euclidiana avaliada em diferentes dimensões. Agora, passando da dimensão m para $m+1$ através do tempo de defasagem, há um novo sistema de coordenadas e como consequência uma nova distância entre $S_{n}$ e $S_{n}^{(\mathrm{r})}$. Quando essas distâncias mudam de uma dimensão para outra, existem falsos vizinhos. A dimensão do espaço apropriada pode ser obtida quando não há mais falsos vizinhos após o aumento da dimensão. Calcula- se o quadrado da distância euclidiana entre esses pontos (SAVI, 2006).

$$
r_{m}^{2}(t, r)=\sum_{k=0}^{m-1}\left[S(t+k \tau)-S^{(r)}(t+k \tau)\right]^{2}
$$

Segundo Kennel et al. (1992) e Savi (2006) a dimensão mínima é encontrada quando as distâncias definida pela Eq. (3.4) entre as dimensões $m$ e $m+1$ param de crescer ou seja, quando a seguinte equação for verdadeira:

$$
\left[\frac{\boldsymbol{r}_{m+1}^{2}(t, r)-\boldsymbol{r}_{m}^{2}(t, r)}{r_{m}^{2}(t, r)}\right]^{\frac{1}{2}}>\boldsymbol{R}_{t o l}
$$

onde $R_{t o l}$ é uma constante que segundo Kennel et al. (1992) para valores $R_{t o l} \geq 10$ os falsos vizinhos são identificados.

\subsection{IDENTIFICAÇÃO DE SISTEMAS}

Esta seção traz uma breve resenha bibliográfica sobre modelagem e identificação de sistemas não-lineares e lineares. Varios sistemas dinâmicos estudados na engenharia na prática são não-lineares (Aguirre, 2000). Entretanto em diversas aplicações, a utilização de representações lineares na modelagem e identificação de tais sistemas apresenta resultados satisfatórios. Existem possibilidades em que os modelos lineares podem ser substituídos por representações não lineares, pois estas são capazes de reproduzir certos fenômenos e comportamentos mais complexos dos sistemas reais. Diante desse fato, observa-se, nas últimas décadas, um interesse crescente no desenvolvimento e utillização de modelos não- 
lineares para caracterizar sistemas dinâmicos. Uma abordagem muito utilizada para descrever sistemas não-lineares é a série de Volterra (Trendafilova et al., 2000).

Para a representação de sistemas não-lineares, além da série de Volterra, destaca-se também os modelos de blocos interconectados. Estes modelos são compostos pela conexão entre dois blocos, sendo que um deles representa uma dinâmica linear e o outro uma nãolinearidade estática (Coelho et al., 2002). A sequência em que os blocos estão conectados caracteriza o modelo como Hammerstein ou Wiener. Devido à simplicidade e também por possibilitarem a utilização de técnicas de identificação lineares já consolidadas, os modelos de blocos interconectados são utilizados em numerosas aplicações.

Uma grande quantidade de publicações apresenta a utilização dos modelos Hammerstein e Wiener. Por exemplo, em Alonge (2003) é apresentado um método para a identificação de um sistema não-linear em que o modelo Hammerstein é utilizado para representar a não-linearidade estática, seguido por um modelo ARX ('auto regressive with exogeneous') representando a parte linear. Greblick e Pawlack (1989) mostraram como identificar um modelo Hammerstein não-linear discreto no tempo. Em Er-Wei Bai (2003) foi proposta uma aproximação no domínio da frequência para modelos Hammerstein. Em Greblick (1992), utiliza-se identificação não-paramétrica para identificar a não-linearidade em modelos de Wiener. Em Vörös (2003) realiza-se a modelagem e a identificação de parâmetros de modelos de Wiener com não-linearidades fortemente assimétricas.

Os sistemas não-lineares, diferentemente dos lineares, não apresentam um modelo matemático geral e sistemático que possa representar várias classes de problemas. Um sistema, linear ou não linear, deve ser entendido como uma mistura de componentes (físicos, biológicos, econômicos, entre outros) que devem interagir e desempenhar uma determinada função (OGATA, 1998; D’AZZO e HOUPIS, 1978). Sistemas que são constituídos por variáveis dependentes do tempo são chamados de sistemas dinâmicos.

A primeira etapa da análise de um sistema dinâmico consiste na sua modelagem matemática. Modelar matematicamente um sistema significa obter uma representação análoga matemática que descreva as características e propriedades principais observadas no sistema. A representação matemática de um sistema é muito útil, pois a partir dela pode-se utilizar ferramentas computacionais para a realização de estudos sobre características dinâmicas, estabilidade, desempenho, estratégias de controle, previsão da dinâmica, análise de períodos, padrões, caos, etc. sem a necessidade de realizar diversos testes práticos.

A determinação do comportamento do sistema que deve ser reproduzido em um modelo depende para qual finalidade o modelo é desejado. Para isso deve-se empregar 
vários tipos de modelos. Os mais comuns são: modelos estáticos e dinâmicos; discretos e contínuos; determinísticos e estocásticos; paramétricos e não-paramétricos. $\mathrm{O}$ modelo matemático é uma representação aproximada de um sistema, e geralmente, se tem mais que um modelo que reproduza tal sistema. Desse modo, torna-se necessária a escolha de um modelo para a representação mais adequada para uma determinada aplicação. Pode-se afirmar que, a obtenção de um modelo matemático satisfatório para as especificações e objetivos desejados é a parte mais importante na modelagem e identificação de sistemas.

Para Aguirre (2000) a construção de um modelo matemático pode-se utilizar diversas técnicas. Uma delas é a modelagem chamada "caixa branca", também conhecida como modelagem fenomenológica ou conceitual. Esse tipo de modelagem se baseia nas propriedades e leis físicas que regem os fenômenos observados no sistema. Para utilizar essa técnica deve-se conhecer bem as características do sistema a ser modelado. Por exemplo, a modelagem matemática de um circuito elétrico analógico pode ser baseada nas leis de Kirchhoff, um sistema mecânico pode ser modelado baseando-se nas leis de Newton.

Quando o acesso à composição física do sistema é difícil, ou este é complexo, a modelagem caixa branca pode se tornar trabalhosa, demorada e, portanto, inviável. Sendo assim, torna-se necessária a utilização de técnicas alternativas que requeiram pouco ou nenhum conhecimento prévio do sistema para a obtenção do modelo. Esse tipo de modelagem é conhecido como modelagem empírica ou "caixa preta". A área do conhecimento que estuda esse tipo de técnicas de modelagem é conhecida como identificação de sistemas.

A identificação de sistemas consiste de um conjunto de procedimentos visando gerar modelos matemáticos de sistemas dinâmicos através de seus dados de entrada e saída. Basicamente a identificação tenta responder à questão: que modelo ao ser excitado por uma entrada u resulta na saída y? (Aguirre, 2000). Assim a identificação de sistemas é o nome dado a área da engenharia que estuda a construção de modelos de sistemas dinâmicos com intuito de simular um problema real. Um modelamento pode ser realizado por princípios básicos a partir de dados observados.

O processo de identificação de sistemas é constituído por 5 etapas descritas a seguir:

1. Coleta de dados - É o processo que se inicia na geração dos dados, cuidando para que esses dados estejam livres de interferências durante a observação da obtenção dos dados de entrada e saída do sistema que serão utilizados para a construção do modelo. É 
importante na realização de experimentos para a coleta de dados que os sinais de excitação e do tempo de amostragem sejam bem escolhidos. Assim as condições experimentais bem projetadas são fundamentais para a qualidade final dos dados adquiridos.

2. Escolha da representação do modelo - Observando as características do sistema a ser modelado, pode-se obter, dentre as diversas possibilidades, uma determinada representação que seja a mais adequada para modelar esse sistema. Como exemplos de representações, pode-se citar: equações diferenciais, funções de transferência, entre outras.

3. Escolha da estruturação do modelo - Após a escolha da representação do modelo, deve se determinar como será a estrutura dessa representação. Isso corresponde a escolher, por exemplo, a dimensão do modelo, ou em uma representação contínua, o número de pólos e zeros em uma função de transferência, ou quantidade de neurônios em uma rede neural, ou valor de atraso da saída no modelo de Hammerstein e Wiener, etc. Essa etapa, juntamente com a anterior é fundamental no processo de identificação de um sistema.

4. Estimação de parâmetros - Esse é o processo que deve determinar valores adequados para os parâmetros que compõem o modelo, com base nas informações resultantes das etapas anteriores. Sendo assim, nessa fase, ocorre a escolha e a utilização de algoritmos para realizar a estimação desses parâmetros. A maior parte dos algoritmos utilizados para esse fim é baseada no método clássico dos mínimos quadrados e suas variantes. A etapa de estimação de parâmetros resulta, então, na definição final de um modelo que represente adequadamente o sistema em questão.

5. Validação do modelo - Com a obtenção de um determinado modelo para o sistema, torna-se necessário verificar se o mesmo é válido. Isto é, se o modelo incorpora as características de interesse do sistema original. O resultado da validação depende da aplicação pretendida para o modelo e da quantidade de informação que se tem do sistema original. Uma maneira comum de se validar modelos é a comparação da simulação do modelo obtido com os dados reais do sistema. Para uma melhor avaliação da qualidade do modelo, utiliza-se conjuntos de dados diferentes para a sua construção e para a sua validação. Pode-se dizer que, de maneira geral, o processo de identificação de sistemas, envolve os seguintes fatores: um sistema, uma condição experimental, uma estrutura de modelo, um método de identificação e um critério de validação. 
O processo de identificação pode ser descrito resumidamente da seguinte maneira: realiza-se um experimento para a coleta de dados do sistema; com base nos dados, escolhese um conjunto de modelos candidatos para a representação do sistema; determina-se qual é o melhor modelo dentro desse conjunto de acordo com as especificações desejadas e realizase testes de validação do modelo. Se ele não for satisfatório, deve-se revisar os passos envolvidos na sua obtenção. Portanto a identificação de sistemas busca a representação do comportamento de um processo por meio de um modelo matemático independente do conhecimento prévio a respeito do mesmo.

O sinal empregado na identificação do sistema deve ser capaz de excitá-lo em toda a faixa de interesse, pois caso contrário, o modelo identificado não será adequado (Ljung, 1999). No caso de um sistema linear, a literatura apresenta diversas técnicas de identificação de sistemas, representadas por equações diferenciais tal como a representação por espaço de estados, onde se observa uma função de transferência que descreve relações de entrada-saída de um sistema, não fornecendo informações mais detalhadas a respeito do que ocorre no interior do sistema. A representação no espaço de estados (diferentemente da F.T) fornece tais informações, pois modela também relações entre as variáveis internas ao sistema. A seguir, são apresentadas algumas definições relacionadas a esse tipo de representação:

- Estado - O estado de um sistema é uma estrutura matemática constituída por um conjunto de $n$ variáveis: $x_{1}(t), x_{2}(t), x_{n-1}(t), x_{n}(t)$ chamadas variáveis de estado, tal que com os valores iniciais $x_{i}\left(t_{0}\right)$, deste conjunto, as excitações do sistema $u_{j}(t)$, e as equações que descrevem sua dinâmica, é possível conhecer os estados futuros e a saída futura do sistema (D’AZZO \& HOUPIS, 1982; DORF \& BISHOP, 2001).

- Variáveis de estado - são as grandezas cujo conjunto de valores determina o estado do sistema (OGATA, 1998). Não é necessário que tais grandezas sejam fisicamente mensuráveis ou observáveis, elas podem ser grandezas puramente matemáticas.

- Vetor de estado - É um vetor n-dimensional $x(t)$, cujos componentes são as $\mathrm{n}$ variáveis de estado necessárias para descrever o comportamento do sistema.

- Espaço de estado - É o espaço n-dimensional cujos eixos de seu sistema de coordenadas são representados pelas componentes do vetor de estado.

- Equações de estado - Conjunto de $\mathrm{n}$ equações diferenciais de $1^{\mathrm{a}}$ ordem, sendo $\mathrm{n}$ o número de variáveis de estado independentes. 
A análise em espaço de estados envolve três tipos de variáveis na modelagem de sistemas dinâmicos: variáveis de entrada, variáveis de saída e variáveis de estado. Deve-se observar que a representação em espaço de estados de um sistema não é única, ou seja, pode-se ter mais de um modelo em espaço de estados para o mesmo sistema. Uma representação linear matricial típica em espaço de estados tem a estrutura definida pelas Eqs. (3.6) e (3.7)

$$
\begin{aligned}
& \frac{d x}{d t}=A x+B u \\
& y=C x+D u
\end{aligned}
$$

A equação (3.6) representa a equação de estado do sistema e a Eq (3.7) é a equação de saída. Os componentes das equações (3.6) e (3.7) são vetores sendo $x(t)$ o vetor de estado $n$-dimensional, com $x \in R^{n}, u(t) \in R^{p}$ é o vetor de entradas formada por $p$ funções temporais, $y(t) \in R^{m}$ é o vetor $m$-dimensional de saídas medidas, $\dot{x}(t)$ é derivada em relação ao tempo, A é matriz de estado ( $n \times n)$, $B$ é a matriz de entrada ( $n \times p)$, $C$ é matriz de saída $(m \times n), \quad$ e $\quad D$ é a matriz de transmissão direta $(m \times p)$. O sistema representado por (3.6) e (3.7) será multivariável se $n>1$ ou $m>1$ e monovariável, caso $p=1$ e $m=1$.

Através da representação em espaço de estados, é possível obter a função de transferência de um sistema (e vice-versa). Lembrando que a F.T relaciona as transformadas de Laplace da saída e da entrada, que aplicada nas equações (3.6) e (3.7), para condições iniciais nulas, resulta em:

$$
\begin{aligned}
& s X(s)-A X(s)=B X(s) \\
& Y(s)=C X(s)+D U(s)
\end{aligned}
$$

Manipulando-se a equação (3.8) e colocando o resultado em (3.9), tem-se:

$$
\begin{aligned}
& (I s-A) X(s)=B U(s) \\
& \left(X(s)(I s-A)^{-1}=B U(s)\right.
\end{aligned}
$$




$$
\begin{aligned}
& Y(s)=C(I s-A)^{-1} B U(s)+D U(s) \\
& G(s)=\frac{Y(s)}{U(s)}=C(I s-A)^{-1} B+D
\end{aligned}
$$

A expressão (3.13) é a função de transferência envolvendo as matrizes $A, B, C$ e $D$. O sistema considerado na Eq.(3.13) pode ser monovariável ou multivariável. No caso de sistemas multivariáveis, a relação $Y(s) / U(s)$ não será apenas a razão entre dois polinômios em s, mas poderá ser representada como a razão entre polinômios de matrizes em $s$ (Aguirre, 2000). Em geral, a representação em espaço de estados é mais conveniente para representar sistemas multivariáveis do que a função ou matriz de transferência.

\subsection{VALIDAÇÃO DO MODELO}

Nessa seção são descritos métodos de validação dos modelos obtidos pelo processo de identificação caracterizando qual é mais adequado à aplicação particular. Uma das formas mais comuns de verificar a validade do modelo é comparar se o modelo é capaz de reproduzir o comportamento do sistema. Para a realização deste teste é importante a utilização de um conjunto de dados diferente daquele empregado na etapa de estimação. Este segundo conjunto de dados, embora diferente, deve ser obtido segundo as mesmas condições de operação do primeiro. Esta prática, embora simples, permite observar a capacidade de generalização do modelo obtido (Ljung, 1999). O Somatório do Erro Quadrático (SSE - Sum of Squared Error) é um índice que representa a soma dos quadrados do erro de estimação e, quanto menor é o seu valor, melhor a qualidade do modelo. A Eq. (3.14) representa a forma de cálculo desse índice.

$$
S S E=\sum_{t=1}^{N}[y(t)-\hat{y}(t)]^{2}
$$

Coeficiente de Correlação Múltipla, representado pela sigla $\mathrm{R}^{2}$, é um método eficaz de validação. O Coeficiente de Correlação Múltipla indica o grau de relacionamento entre as variáveis independentes. A Correlação Múltipla não é simplesmente a soma de correlações da variável dependente com as independentes tomadas separadamente (GUILFORD, 1950). 
Uma das razões é que as variáveis independentes são normalmente intercorrelacionadas, conhecidas também como multicolineares. Quando as intercorrelações forem iguais a zero, então o quadrado do coeficiente de correlação múltipla será a soma dos quadrados dos coeficientes de cada variável independente com a dependente. A Correlação Múltipla aumenta quando aumenta o tamanho da correlação entre as variáveis dependentes e independentes e quando o tamanho das intercorrelações entre as variáveis independentes diminui (GUILFORD, 1950). Da mesma forma que a análise de correlação simples e a regressão simples estão relacionadas, a correlação e regressão múltipla também estão.

$$
R^{2}=1-\left[\frac{\|y(t)-\bar{y}(t)\|}{\|y(t)-\hat{y}(t)\|}\right]^{2}
$$

onde $\bar{y}$ é a média das $N$ amostras medidas para a saída do processo e $\hat{y}$ é série produzida pela predição de determinados modelos impostos. Quando o valor de $R^{2}$ é igual à unidade indica uma exata adequação do modelo para os dados medidos do processo. Um valor de $R^{2}$ entre 0.9 e 1 é considerado suficiente para aplicações práticas, em sistemas de controle (COELHO e COELHO, 2004).

Estes índices de desempenho não levam em consideração a complexidade (número de parâmetros) do modelo, embora forneçam uma indicação importante em relação à qualidade do modelo obtido. A seleção do modelo mais adequado deve sempre levar em conta a relação entre capacidade de representação e simplicidade estabelecendo um compromisso entre precisão do modelo versus esforço computacional, por exemplo, por meio do critério de informação de Akaike (LJUNG e GLAD, 1994).

O toolbox 'identification system' do Matlab® calcula o teste FIT em uma de suas ferramentas e é descrita por Ljung (1999). Essa qualidade de ajuste entre os dados de teste e de referência é um critério Normalized Root Mean Square error (NRMSE) e é utilizado para gerar o número de ajuste em porcentagem. É uma medida de quanto melhor o modelo é em reproduzir os dados relativamente observadas para a média dos dados.

$$
\mathrm{FIT}=1-\left[\frac{\|y(t)-\bar{y}(t)\|}{\|y(t)-\hat{y}(t)\|}\right]
$$

onde $y(t)$ é a série temporal, $\bar{y}(t)$ é a série predita e $\hat{y}(t)$ é a média de Y. 


\subsection{TESTE 0-1}

\section{ANÁLISE DE SÉRIES TEMPORAIS}

Nesta seção é apresentado o método teste 0-1 que foi proposto por Gottwald e Melboune (2005) e (2006) sendo aplicado em séries temporais, com base nas propriedades estatísticas de uma única coordenada. Basicamente, o teste 0-1 consiste em estimar um único parâmetro $K$. $O$ teste considera uma variável do sistema $x_{j}$, onde duas novas coordenadas $(p, q)$ são definidas como se segue em eq. 3.17 a equação coordenada p e em eq. 3.18 a equação coordenada q.

$$
\begin{aligned}
& p(n, \bar{c})=\sum_{j=0}^{n} x(j) \cos (j \bar{c}) \\
& q(n, \bar{c})=\sum_{j=0}^{n} x(j) \sin (j \bar{c})
\end{aligned}
$$

onde, $\bar{c} \in(0, \pi)$ é uma constante. O desvio quadrático médio das novas variáveis $p(n, \bar{c})$ e $q(n, \bar{c})$ é dado por:

$$
M(n, \bar{c})=\lim _{N \rightarrow \infty} \frac{1}{N} \sum_{j=1}^{N}\left[(p(j+n, \bar{c})-p(j, \bar{c}))^{2}+(q(j+n, \bar{c})-q(j, \bar{c}))^{2}\right]
$$

onde $n=1,2, \ldots, N$ e, portanto, obtém-se o parâmetro $K_{c}$ no limite quando o tempo tende a um valor muito alto.

$$
K=\frac{\operatorname{cov}(\mathrm{Y}, \mathrm{M}(\bar{c}))}{\sqrt{\operatorname{var}(\mathrm{Y}) \operatorname{var}(\mathrm{M}((\bar{c}))}}
$$

onde os vetores $M(\bar{c})=\left[M(1, \bar{c}), M(2, \bar{c}), \ldots, M\left(n_{\max }, \bar{c}\right)\right]$ e $Y=\left[1,2, \ldots, n_{\max }\right]$. Dado dois vetores $x$ e $y$, a covariância $\operatorname{cov}(x, y)$ e variância $\operatorname{var}(x)$, de $n_{\max }$ elementos, são definidas, respectivamente por:

$$
\operatorname{cov}(x, y)=\frac{1}{n_{\max }} \sum_{n=1}^{n_{\max }}(x(n)-\bar{x})(y(n)-\bar{y})
$$




$$
\operatorname{var}(x)=\operatorname{cov}(x, x)
$$

onde $\bar{x}$ e $\bar{y}$ são as médias de $x(n)$ e $y(n)$, respectivamente. Como resultado final, o valor do parâmetro $K$ é obtido fazendo a mediana dos $m$ diferentes valores de $\bar{c} \in(0, \pi)$ na Eq. (3.20). De acordo com Gottwald e Melboune (2005) se o valor $K$ está perto de 0 o sistema é periódico, por outro lado, se $K$ é perto de 1 o sistema é caótico.

\subsection{ESCALA INDEXADA}

A transformada wavelet de um sinal unidimensional consiste na sua expansão em relação a uma base construída por meio de funções chamadas wavelets, com várias transformações internas e de deslocamentos (PERMANN e HAMILTON, 1992; AWREJCEWICZ et al. , 2011).

Um comportamento não-periódico significa que existem trajetórias que não se estabelecem em órbitas periódicas ou quase periódicas para $t \rightarrow \infty$ (STROGATZ, 1994). Dado $f \in L^{2}(\mathbb{R})$, a transformada continua de wavelet de $f$ no tempo $u$ e escala $s$ é definida como:

$$
W f(u, s):=\left\langle f, \psi_{u, s}^{*}\right\rangle=\int_{-\infty}^{+\infty} f(t) \psi_{u, s}^{*}(t) d t
$$

onde

$$
\psi_{u, s}^{*}=\frac{1}{\sqrt{s}} \psi\left(\frac{t-u}{s}\right), u \in \mathbb{R}, s>0
$$

e $W f(u, s)$ proporciona o componente de frequência (ou detalhe relevante) de um sinal de $f$ no tempo $u$ e escala $s$ com relação à analise de wavelet $\psi_{u, s}$ (BENÍTEZ et al., 2010). O escalograma de $f$,, é definido por (BENÍTEZ et al., 2010):

$$
\wp(s):=\|W f(u, s)\|=\left(\int_{-\infty}^{+\infty}|W f(u, s)|^{2} d u\right)^{1 / 2}
$$

$\wp(s)$ é a energia da wavelet de $f$ na escala $s$. O escalograma é uma ferramenta útil usada para estudar o sinal, uma vez que oferece a possibilidade de detectar suas escalas (ou 
frequências) mais representativas. O escalograma interior de $f$ na escala de $s$ pode ser definida por (BENÍTEZ et al., 2010):

$$
\wp^{\text {inner }}(s):=\|W f(u, s)\|_{J(s)}=\left(\int_{c(s)}^{d(s)}|W f(u, s)|^{2} d u\right)^{1 / 2}
$$

onde $J(s)=[c(s), d(s)] \subseteq I$ é o máximo do sub intervalo em $I$ para o suporte de $\psi_{u, s}$ que está incluído em $I$ para todos $u \in J(s)$, onde $I$ é um intervalo de tempo finito (BENÍTEZ et al., 2010). O comprimento de $J(s)$ depende da escala $s$, de modo que o valor do escalograma interior nas diferentes escalas não podem ser comparados. Logo, escalograma interior devem ser normalizadas como se segue (BENÍTEZ et al., 2010).

$$
\bar{\wp}^{\text {inner }}(s)=\frac{\wp^{\text {inner }}(s)}{(d(s)-c(s))^{1 / 2}}
$$

A escala indexada no interval de escala $\left[s_{0}, s_{1}\right]$ é definida pelo quociente (BENÍTEZ et al., 2010).

$$
i_{\text {scale }}:=\frac{\wp\left(s_{\min }\right)}{\wp\left(s_{\max }\right)}
$$

onde $s_{\max }$ e $s_{\min }$ são pequenas escalas no intervalo $\wp\left(s_{\min }\right) \leq \wp(s) \leq \wp\left(s_{\max }\right)$ para todo $s \in\left[s_{0}, s_{1}\right]$. Note-se que a escala indexada é considerada somente para sinais compactados que suportam o escalograma interior normalizado (BENÍTEZ et al., 2010). Desta definição, a escala indexada $i_{\text {scale }}$ está entre $0 \leq i_{\text {scale }} \leq 1$ e isso pode ser interpretado como uma medida de grau não periodicidade de sinal: a escala indexada será zero ou perto de zero para as sequências periódicas e perto de um para as sequências altamente não periódicas (BENÍTEZ et al., 2010).

Em anos recentes, alguns sistemas clássicos, tais como o mapa logístico, o mapa de Henon (BENÍTEZ et al., 2010), o Bonhoeffer-van der Pol oscilador, o sistema de Rössler (AKHSHANI et al., 2014), (GOTTWALD e MELBOURNE, 2004) e a dinâmica de batimentos cardíacos (BEHNIA et $A l ., 2013$ ) entre outros, foram analisados pelo índice de escala. 


\section{CAPÍTULO 4}

\section{MODELAGEM MATEMÁTICA E RECONSTRUÇÃO DO ESPAÇO DE ESTADOS}

Neste capítulo é apresentado o modelo matemático utilizado na presente tese. De acordo com Wang, Pai e Yau (2009) a microviga do AFM pode exibir um movimento caótico em certas condições de análise e parâmetros escolhidos para varredura. Esta questão tem sido experimentalmente observada por Raman et al. (2003) e por Burnhan (1993).

Estudos teóricos, com base nas técnicas da teoria de Melnikov, foram realizados por Ashhab et al. (1999) para provar a existência de conjuntos de invarianças caóticas e para determinar a região no espaço de parâmetros físico onde movimento caótico está presente.

Em 2006, Hu e Raman utilizando experiências sistemáticas em uma variedade de microvigas em uma ampla gama de condições operacionais e mostrou que as respostas dinâmicas de AFM das microvigas podem ter bifurcações periódicas às oscilações caóticas perto da transição do não-contato e do modo intermitente.

O comportamento caótico em AFM é altamente indesejável e traz efeitos negativos uma vez que este tipo de movimento irregular faz com que o AFM funcione medindo imprecisamente e tornando baixa a resolução da topografia da amostra. Para garantir o bom desempenho do microscópio e eliminar a possibilidade do movimento caótico das microvigas, é necessário alterar as condições de funcionamento do AFM para uma região, em que os parâmetros façam o movimento ser regular ou então projetar um controlador ativo que estabilize o sistema em uma de suas órbitas periódicas estáveis. Em 1999, Ashhab et al. aplicaram um controlador proporcional e derivativo dos AFMs que calcula a função de Melnikov, em termos dos parâmetros do controlador. Usando essa relação, é possível projetar controladores que vai eliminar a possibilidade de caos em AFMs.

No trabalho de Wang et al. (2009) os autores traçam uma estratégia de controle desenvolvida para a supressão de caos, mesmo com as incertezas do modelo e as perturbações externas. Os resultados mostraram que as vibrações não síncronas do sistema de AFM pode ser suprimida através da implementação de um controle de malha aberta. $\mathrm{O}$ esquema proposto pode ser aplicado a outros tipos de sistemas de AFM. A dinâmica não linear do sistema descreve a operação não contato do AFM e é obtida através do modelo 
proposto por Wang et al. (2008) e Ashab et al. (2008) e também pela inclusão da não linearidade cúbica (YABUNO et al., 2008) e (HORNSTEIN et al., 2008).

A microviga é a principal parte para o regime de detecção utilizada pelo microscópio de força atômica e interage com a amostra através da ponteira da sonda que está montada sobre ela. A interação entre a amostra e a microviga do AFM é modelada com base no trabalho de Wang et al. (2009) e é ilustrada na Fig. 4.1.

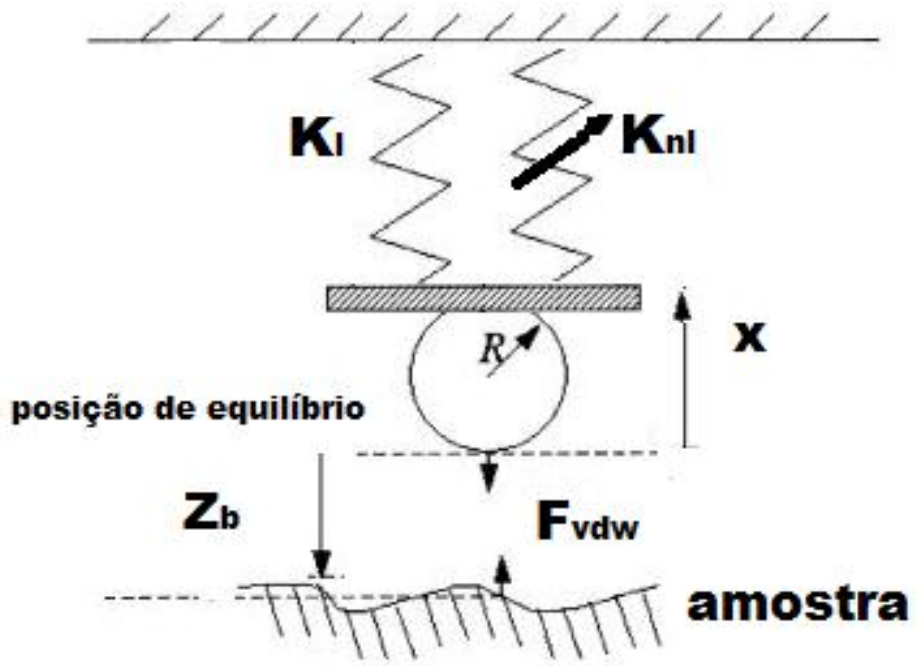

Figura 4. 1 - Modelo da ponteira da microviga do AFM

A equação de movimento da ponteira da microviga do AFM é dada por:

$$
m \ddot{X}+k_{n l} X^{3}+k_{l} X=F_{v d w}+f \cos (w t)
$$

onde $F_{v d w}$ é a força de Van der Waals, onde a força é de regime repulsiva, expressa por (ISRAEACHVILI, 2005):

$$
F_{v d w}=-\frac{A R}{6\left(Z_{b}+X\right)^{2}}
$$

e $m$ é a massa da microviga, $A$ é a constante de Hamaker que é a variável de interação intermolecular entre o material da microviga e da amostra, $Z_{b}$ é a altura de equilíbrio fixada do suporte da microviga até a amostra, $X$ são as deflexões na indicada na Fig 4.1, $f$ é a amplitude da excitação, $R$ é o raio da esfera da ponteira e $k_{l}$ é a rigidez linear e $k_{n l}$ é a rigidez não linear da microviga. 
De um modo geral, os modelos matemáticos de AFM apresentam parâmetros que variavelmente ocasionam movimentos caóticos. Na equação 4.1 a não linearidade causada pela força de Van der Waals no modo repulsivo é um agente causador do movimento caótico.

A dinâmica não-linear das microvigas dos AFMs durante o processo de varredura é altamente não-linear e tem sido investigada por vários autores na literatura atual. A definição do modelo, a se executar a identificação no capítulo 6 , é baseada no fato de ser um oscilador massa mola adaptado ao funcionamento do AFM que reproduz com um grau de liberdade, o que facilita sua manipulação algébrica. Segundo (WANG et al., 2009), (GARCIA et al., 2000), (JALILI e LAXMINARAYANA, 2004, (HU e RAMAN, 2007), (RAMAN et al. 2008), (LOZANO e GARCIA, 2008) e uma série de outros trabalhos publicados na literatura atual, os modelos matemáticos que regem a dinâmica do AFM são formulados pelo uso do princípio de Hamilton, obtendo, de acordo com os autores citados, as equações governantes do movimento.

A rigidez total é dada por $k=k_{l}+k_{n l}$ e a função potencial do sistema usando a Eq. (4.2) é escrita como:

$$
V=-\frac{A R}{6\left(Z_{b}+X\right)}+\frac{1}{2} k_{l} X^{2}+\frac{1}{4} k_{n l} X^{4}
$$

A energia mecânica total por unidade de massa é expressa por:

$$
\begin{aligned}
& E=\frac{\text { energia }}{\text { massa }}=\frac{T+V}{m}=\frac{1 m}{2 m} \dot{X}+\frac{1 k_{l}}{2 m} X^{2}+\frac{1 k_{n l}}{4 m} X^{4}-\frac{A R}{6 m\left(Z_{b}+X\right)} \\
& E=\frac{1}{2} \dot{X}+\frac{1}{2} \omega_{1}^{2} X^{2}+\frac{1}{4} \omega_{2}^{2} X^{4}-\frac{D \omega_{1}^{2}}{\left(Z_{b}+X\right)}
\end{aligned}
$$

onde $\omega_{1}=\sqrt{\frac{k_{l}}{m}}$ é a frequência de primeira ordem, $\omega_{2}=\sqrt{\frac{k_{n l}}{m}}$ e $D=\frac{A R}{6 k_{l}}$. Considerando a Eq. (4.4) e Eq.(4.5) e as variáveis de estado $X_{1}=X$ e $X_{2}=\dot{X}$, obtém se a equação do movimento em espaço de estados:

$$
\left\{\begin{array}{c}
\dot{X}_{1}=X_{2} \\
\dot{X}_{2}=-\omega_{1}^{2} X_{1}-\omega_{2}^{2} X_{1}^{3}-\frac{D \omega_{1}^{2}}{\left(Z_{b}+X_{1}\right)^{2}}+f \cos \omega t
\end{array}\right.
$$


Adimensionalizando a eq. (4.6) obtém-se:

$$
\left\{\begin{array}{c}
x_{1}^{\prime}=x_{2} \\
x_{2}^{\prime}=-a_{1} x_{1}-a_{2} x_{1}^{3}-\frac{b}{\left(z+x_{1}\right)^{2}}+c \cdot \cos \tau
\end{array}\right.
$$

onde a linha representa a derivada em relação ao tempo adimensional $\tau$ e:

$$
\tau=\omega t, \quad x_{1}=\frac{X_{1}}{Z_{s}}, \quad z=\frac{Z_{b}}{Z_{s}}, \quad a_{1}=\frac{\omega_{1}^{2}}{\omega^{2}}, \quad a_{2}=\frac{\omega_{2}^{2} Z_{s}^{2}}{\omega^{2}}, b=\frac{D \omega_{1}^{2}}{\omega^{2} Z_{s}^{3}}, c=\frac{f}{\omega^{2} Z_{s}} \text { e } Z_{s}=\left(\frac{D a_{1}}{b}\right)^{\frac{1}{3}}
$$

com $X_{1}$ a deflexão da microviga, $Z_{s}$ a distância do suporte da microviga até a amostra. A fig.4.2 mostra o retrato de fase da equação (4.7) modelada com $\tau=400$ e passo de tempo de 0.1 com $\mathrm{b}=0.02173, \mathrm{c}=2.6364, \mathrm{z}=2.5, a_{1}=0.14668$ e $a_{2}=2.1269$ e integrador Runge-Kutta de ordem 4 e 5.

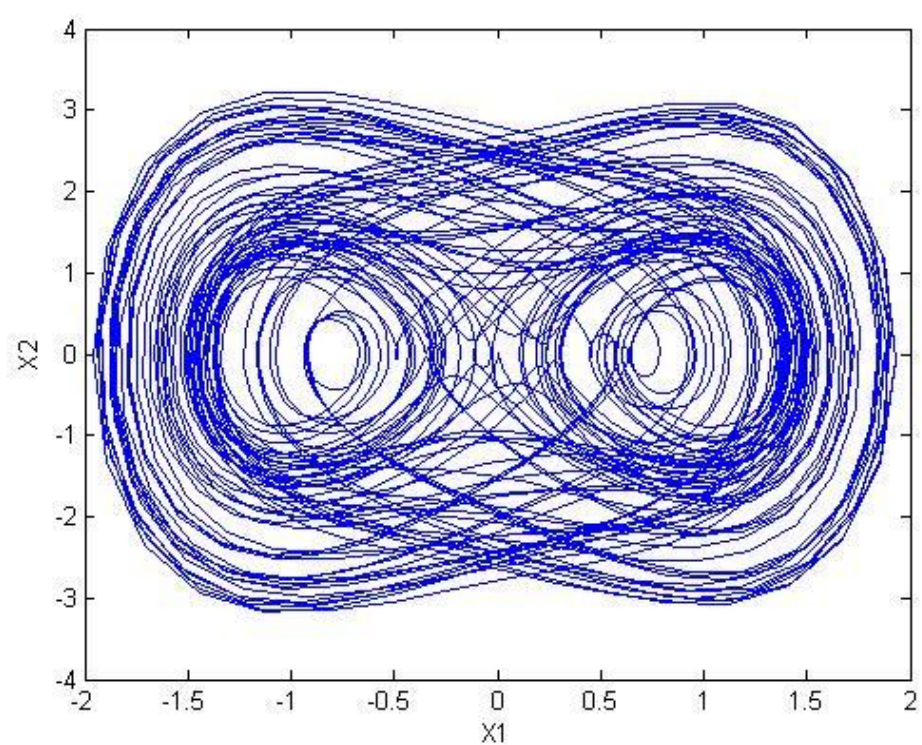

Figura 4. 2- O retrato de fase da equação modelada com 4000 pontos

A figura 4.2 é o mapa construído a partir de $x 1$ e $x 2$ da equação diferencial do modelo matemático. Para a próxima etapa, a reconstrução do espaço de estados é feita seguindo a ideia de que é um processo livre de ruídos. Para isso, um procedimento proposto por Kennel (1992) e representado no diagrama da Fig 4.2 por Viola (2011): 


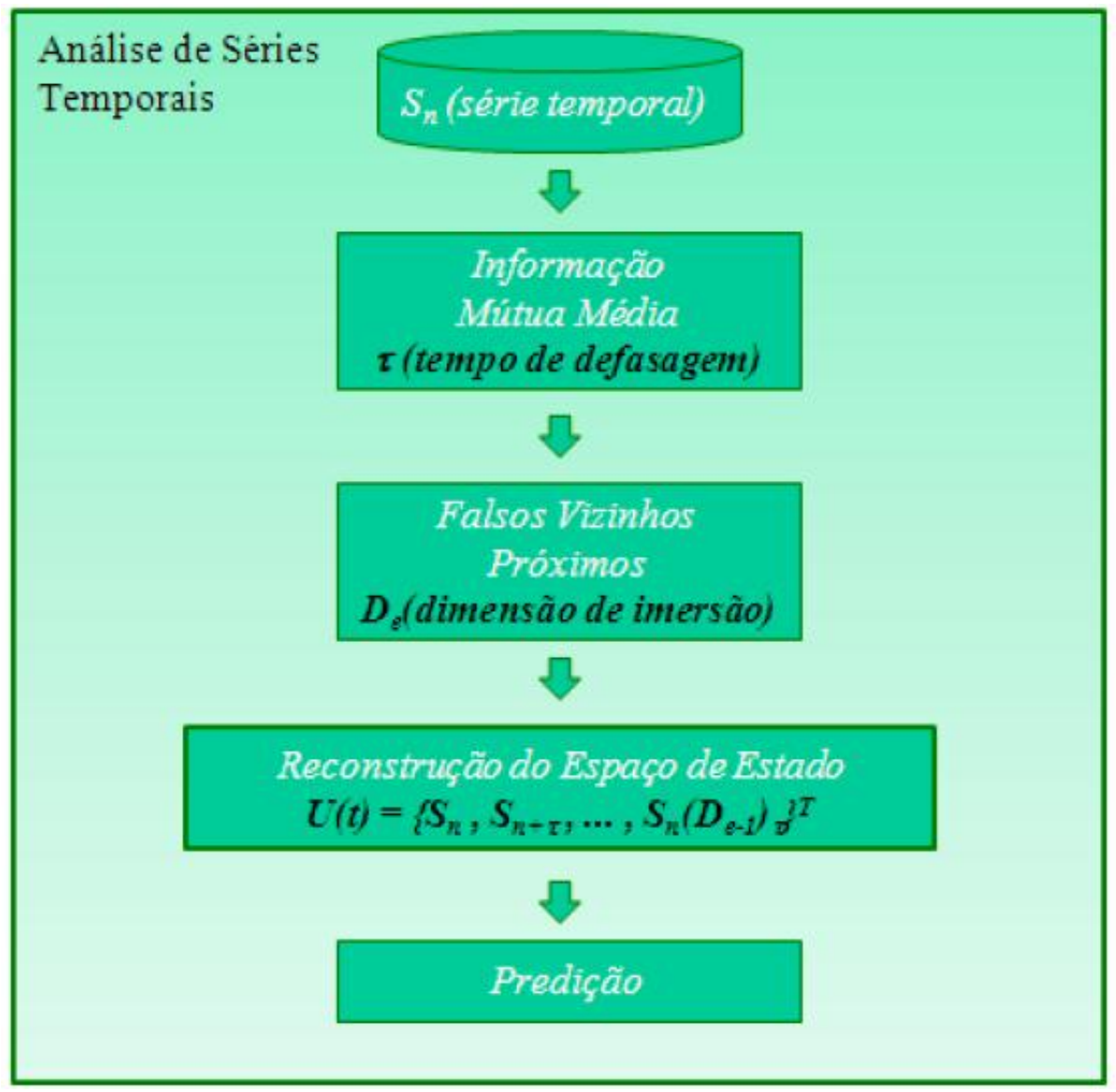

Figura 4. 3 - Esquema proposto por Viola (2011)

A reconstrução do espaço de estados pela técnica das coordenadas defasadas é feita para se comparar o retrato de fase da equação modelada e a reconstruída. A série $S n$ é a saída x1 da equação diferencial e deve-se calcular a informação mútua para obter o atraso, que é o valor correspondente com o mínimo local da informação mútua.

Tabela 1- Informação mútua para série obtida pelo modelo AFM

\begin{tabular}{|c|l|}
\hline$\tau$ & Informação mútua \\
\hline 0 & 2.69237600000000 \\
\hline 1 & 1.66964500000000 \\
\hline 2 & 1.19818300000000 \\
\hline 3 & 0.879955400000000 \\
\hline 4 & 0.682286700000000 \\
\hline 5 & 0.550938100000000 \\
\hline 6 & 0.473223800000000 \\
\hline 7 & 0.442970000000000 \\
\hline 8 & 0.465785600000000 \\
\hline 9 & 0.471815700000000 \\
\hline
\end{tabular}




\begin{tabular}{|l|l|}
\hline 10 & 0.508045500000000 \\
\hline 11 & 0.531620500000000 \\
\hline 12 & 0.536630800000000 \\
\hline 13 & 0.485936100000000 \\
\hline 14 & 0.425856500000000 \\
\hline 15 & 0.349872300000000 \\
\hline 16 & 0.292351500000000 \\
\hline 17 & 0.276152900000000 \\
\hline 18 & 0.267476800000000 \\
\hline 19 & 0.253575100000000 \\
\hline 20 & 0.265487800000000 \\
\hline
\end{tabular}

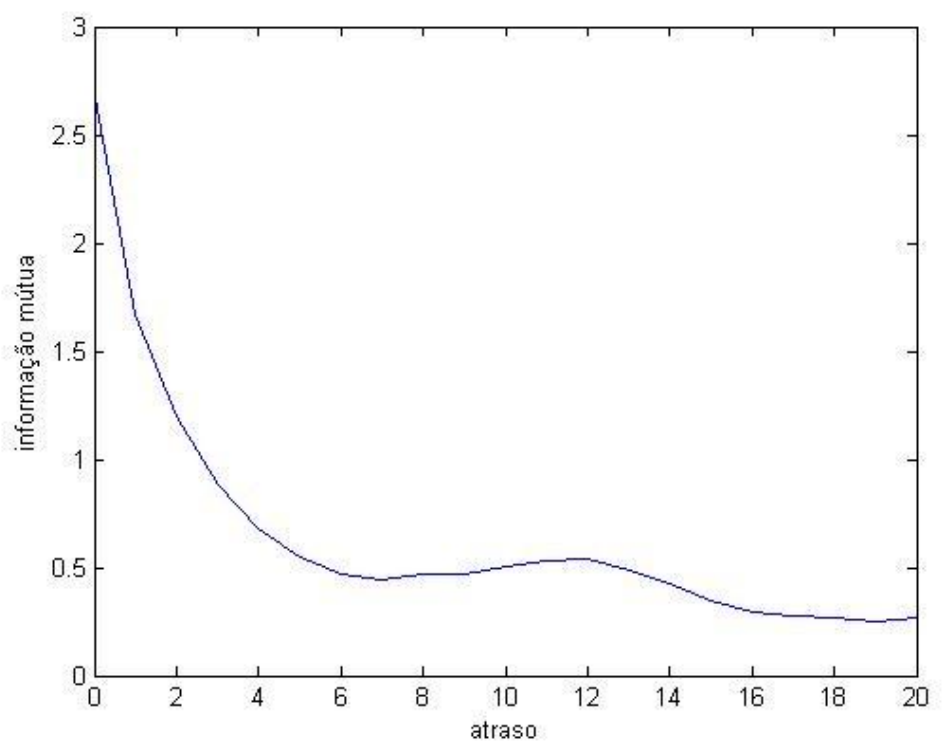

Figura 4. 4 - Informação mútua da série produzida pela equação modelada

Sendo $I(7)=4.429700 \mathrm{e}-001$, mínimo global da informação mútua, o atraso para a formação da nova série é 7 , calcula-se a dimensão em que a quantidade mínima de falsos vizinhos próximos é zero. 


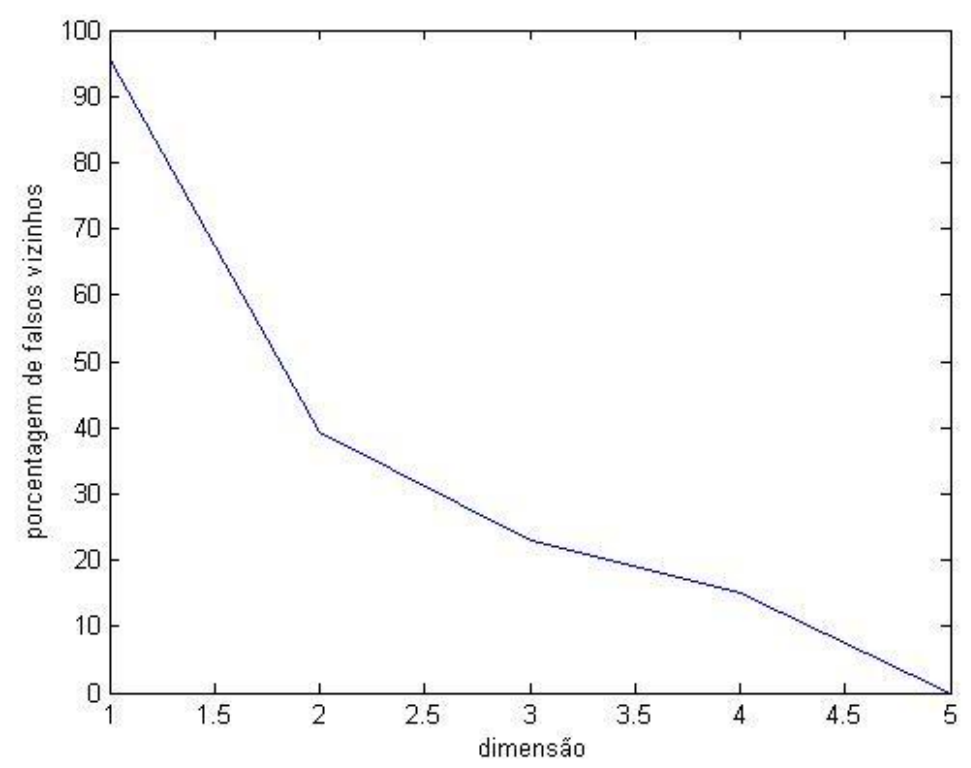

Figura 4. 5 - Porcentagem de falsos vizinhos calculada para a série produzida pela equação modelada

De acordo com a Fig. 4.4 a porcentagem de falsos vizinhos é de aproximadamente 97\% quando a série está inserida em uma única dimensão, quando a dimensão de imersão é aumentada para 5, não há falsos vizinhos. Na Fig 4.5 o atrator caótico é reconstruído e é apresentado em seu retrato de fase com atraso de 7 e com 5 dimensões de imersão.

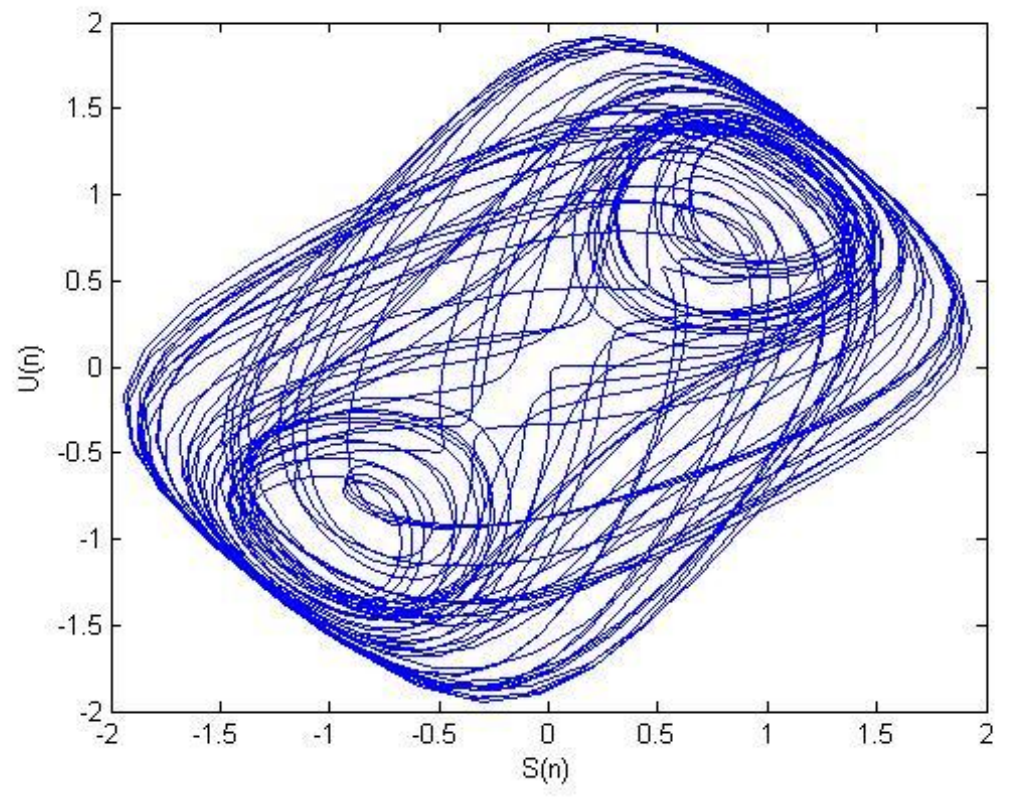

Figura 4. 6 - Retrato de fase da série temporal reconstruída

A próxima etapa proposta no diagrama, predição das séries temporais, não será executada nesta tese, por que não há necessidade de se coletar pontos preditos. Caso houvesse necessidade de uma quantidade maior de pontos, no caso do experimento, bastaria 
fazer o escaneamento e coletar diretamente no aparelho AFM. No caso do modelo de equação diferencial, bastaria aumentar o tempo de integração, assim obtendo uma série maior. Sendo 3000 pontos na série temporal, na equação diferencial e no experimento, um número suficiente para a análise dos resultados propostos no capítulo 5. 


\section{CAPÍTULO 5}

\section{PROCESSO EXPERIMENTAL E ANÁLISE DOS RESULTADOS}

No presente capítulo é apresentado o procedimento experimental de coleta de dados durante o funcionamento de um microscópio de força atômica. São apresentados resultados para o AFM trabalhando em modo 'soft tapping' nas condições de não contato e contato. $\mathrm{O}$ AFM faz o escaneamento da superfície do material ao longo de uma trajetória retilínea e em uma posição fixa (ponto), descritos respectivamente nas seções 5.1 e 5.2.

A figura 5.1 ilustra a operação do AFM no modo contato e não contato. O modo contato não é útil pelo fato de não haver as deflexões necessárias para o estudo da dinâmica não linear, mas as suas séries são úteis para observar o formato da superfície. Neste modo, a sonda arrasta a superfície e o laser detecta a altura e no software é formada a imagem. $\mathrm{Na}$ figura 5.1 a microviga tem a distância ponta da sonda até a amostra igual a zero, isto é $z=$ 0. Quando o AFM trabalha em modo contato, a microviga arrasta sobre a superfície e assim pode estragar a amostra caso ela seja uma célula ou materiais frágeis. É recomendado o uso deste modo para análise de materiais duros. Na imagem à direita da Fig. 5.1 é possível perceber a altura z e a deflexão caracterizando o modo não contato. No modo não contato, a microviga não chega a tocar a amostra, pois a força de Van der Waals a repele quando a microviga chega próxima à amostra. A partir da coordenada $\mathrm{z}=0$ do modo contato é possível definir a altura fixa da microviga para vibrar em modo não contato. $\mathrm{O}$ modo não contato permite alta resolução de imagem topográfica de superfícies de amostras. O modo "soft tapping" é definido quando o AFM trabalha combinando os dois modos descritos anteriormente, isto é, o modo contato com o não contato. O modo de "soft tapping' supera os problemas associados com atrito, de aderência, as forças electrostáticas, colocando alternadamente a ponta em contato com a superfície para proporcionar alta resolução e, em seguida, o levantamento da ponta para fora da superfície evita que a ponta se arraste em toda a superfície. 

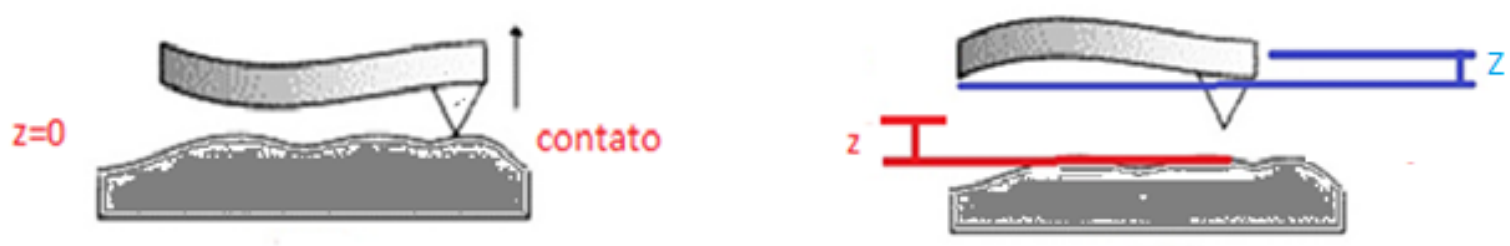

Figura 5. 1 - AFM trabalhando em modo 'soft tapping' nas condições de contato e não contato

Os ensaios experimentais utilizados na presente tese foram realizados no laboratório de microscopia de força atômica do Instituto de Física da Universidade de São Paulo no campus de São Carlos. As figuras 5.2-5.5 descrevem parte do processo experimental. Na Fig. 5.2 uma amostra de safira está sendo preparada pelo técnico do laboratório com a colocação do material em uma lamina plástica. A Fig.5.3 ilustra através de uma visão amplificada o suporte que fixa a ponteira da microviga. A Fig.5.4 mostra o microscópio de força atômica já com a microviga instalada para a realização dos experimentos. A fig 5.5 apresenta uma visão completa do microscópio de força atômica que deve ser calibrado para a realização dos ensaios. Para um bom funcionamento do AFM devem ser considerados os fatores externos ao aparelho: os ambientais, como umidade e temperatura, e vibrações provenientes do entorno onde está localizado o aparelho, que podem vir a causar interferências. Pequenos ruídos interferem na vibração da microviga, como conversas de pessoas, passos pelo laboratório, entre outros.

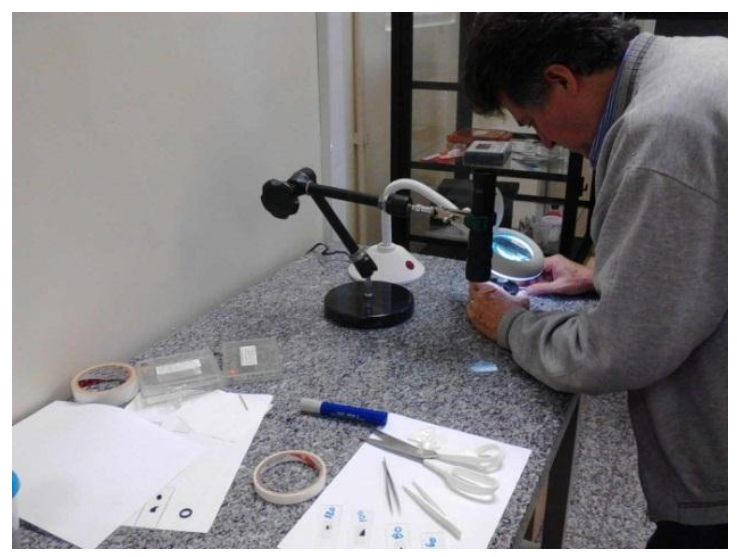

Figura 5. 2 - Preparação da amostra 


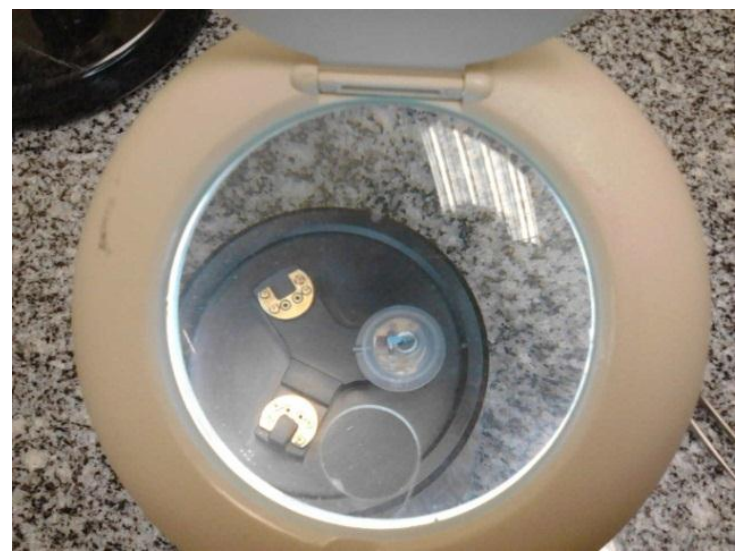

Figura 5. 3 - Visão ampliada do suporte que fixa o tip

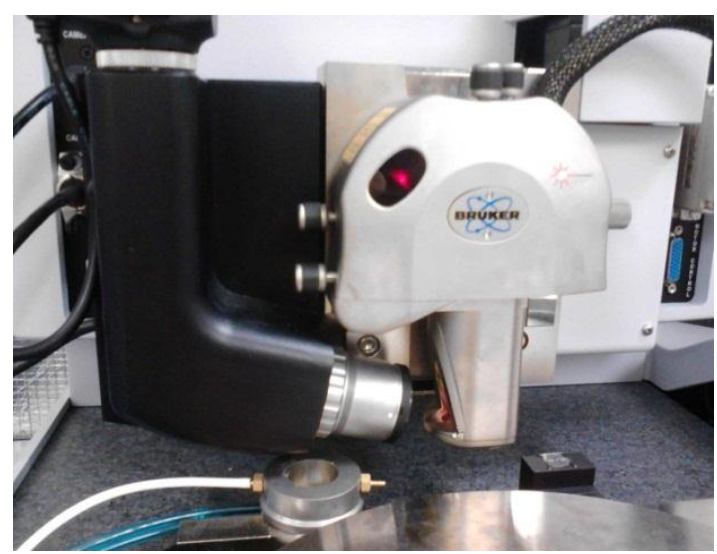

Figura 5. 4 - AFM com o tip instalado

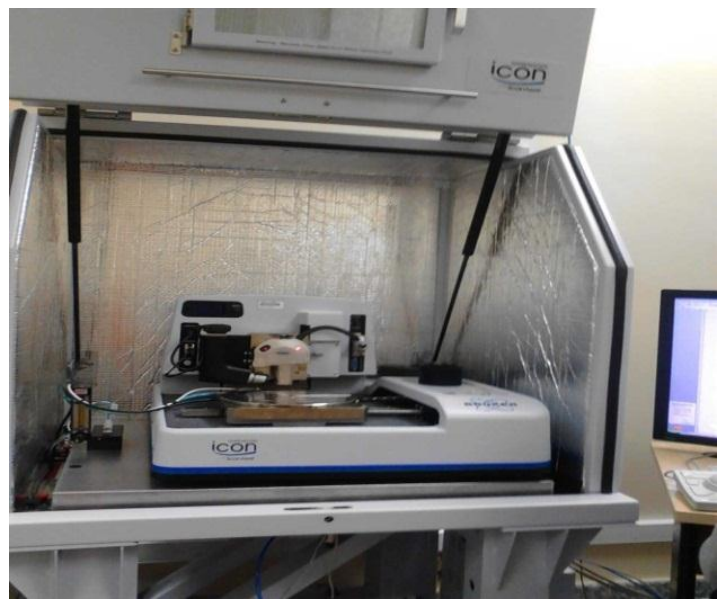

Figura 5. 5 - Calibração do microscópio de força atômica

Os dados experimentais foram obtidos em modo não contato em cinco configurações: (a) amostra de safira com a ponteira da microviga no formato retangular; (b) amostra de safira com ponteira da microviga no formato triangular; (c) amostra de borracha com ponteira da microviga no formato triangular; (d) amostra de safira com ponteira da 
microviga no formato triangular em nitrogênio gasoso; (e) amostra de safira com ponteira triangular em meio líquido (água). Os dados experimentais consistem em um conjunto de pontos que são medidas de deslocamento da microviga em função do tempo. Cada conjunto de pontos em uma das cinco configurações anteriores forma uma série temporal. As séries temporais do AFM foram obtidas através de um microscópio da marca Veeco® e analisadas usando o software nanoscope $®$ ( citar)

O AFM contém um piezelétrico que produz uma excitação externa harmônica. O piezelétrico faz com que a microviga, com oscilação de alta amplitude, mova em direção à superfície até que comece a tocá-la levemente. Durante a digitalização, a ponteira da microviga na posição vertical oscila alternadamente fazendo contatos com a superfície a uma frequência da ordem de 50 mil até 500 mil ciclos por segundo. À medida que a microviga começa a oscilar e contatar a superfície de forma intermitente a frequência de oscilação da microviga é reduzida devido à perda de energia causada pelo contato da sua ponteira com a superfície. A redução da amplitude de oscilação é utilizada para identificar e medir características de superfície.

No experimento, as séries temporais foram adquiridas com a variação da altura da microviga entre a amostra e a ponta é de $\mathrm{z}=0 \mathrm{~nm}$ até $\mathrm{z}=648 \mathrm{~nm}$. A preparação das amostras a serem analisadas pelo AFM é um trabalho minucioso, e requer habilidades, pois o suporte da microviga é muito pequeno, frágil e caro.

A seguir são apresentadas as seções 5.1 e 5.2. Na seção 5.1 são descritos resultados do AFM realizando o escaneamento da superfície da amostra em uma trajetória retilínea. $\mathrm{Na}$ seção 5.2 são discutidos resultados das séries temporais obtidas pelo AFM trabalhando em um ponto fixo.

\subsection{ESCANEAMENTO DO AFM EM MOVIMENTO RETÍLINEO}

Nesta seção foram separadas as séries temporais produzidas varrendo uma região em linha reta representada na Fig 5.7. Foram obtidas 37 séries de 3000 pontos que descrevem a superfície da linha escaneada em diversar alturas. O experimento foi feito com amostra de safira, constante elástica da microviga de $42 \mathrm{~N} / \mathrm{m}$, ponteira padrão de formato retangular 'Sens. amp/sens' de $100 \mathrm{~nm} / \mathrm{V}$, amplitude 'setpoint' de $178.85 \mathrm{mV}$, 'drive setpoint' de 48.52 $m V$, e temperatura da sala de $22.1^{\circ} \mathrm{C}$ 


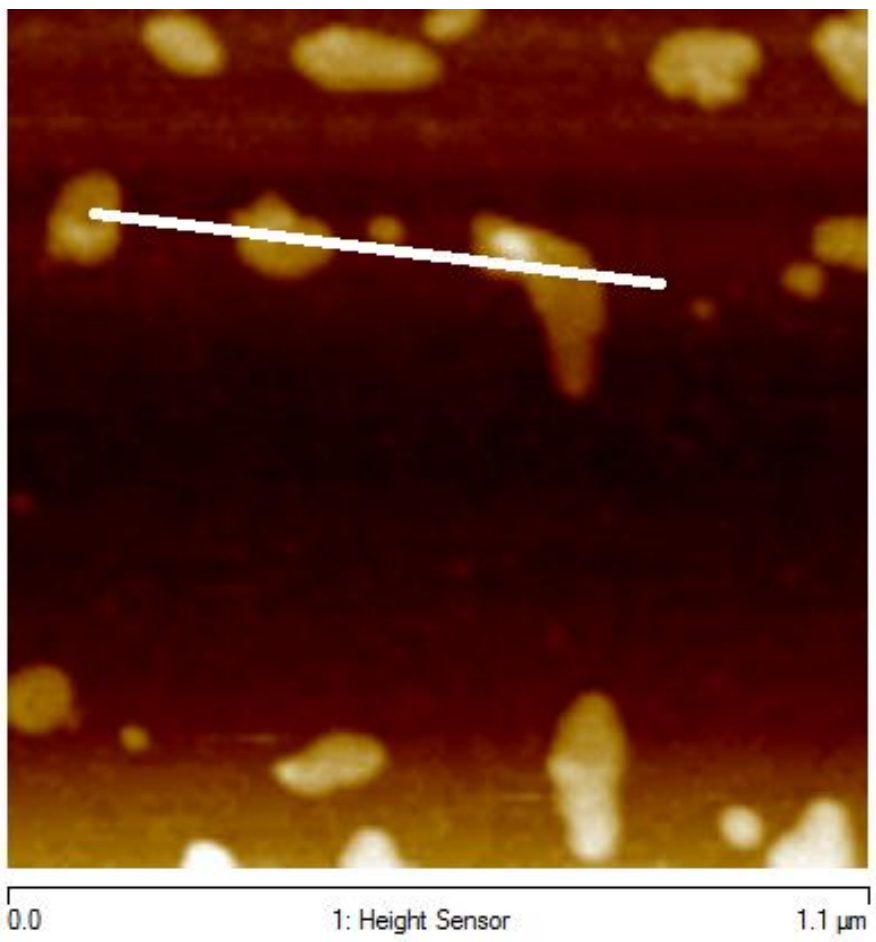

Figura 5. 6 - Trajeto em linha do escâner do AFM na amostra de safira

A Fig. 5.6 mostra o trajeto do escâner que foi escolhido para estar em cima das superfícies com cores as mais diversas variedades de cores que indicam as profundidades. Poderia ter sido escolhida diversas trajetórias em linha, mas a ideia seria mostrar como um em linha as séries seriam produzidas. Na figura 5.7 estão caracterizadas em diversas cores as imagens da superfície da amostra de safira produzidas através do modo 'soft tapping'. Em cor vermelha foi plotado o gráfico do relevo da linha escaneada onde o ponto em que a altura da microviga foi zero, isto é, onde houve o contato entre ponta e amostra e em não contato as demais cores que foram escaneadas por diversas alturas da microviga. Importante notar a velocidade de vibração da microviga. Todas as séries temporais independente da altura da ponta a amostra fixada, tem as deflexões geradas a partir de 1 milisegundo em 1 milisegundo, o que mostra a sua alta velocidade de escaneamento. A figura 5.8 é a imagem em 2D na superfície de safira ampliada em 0.1 segundos. 


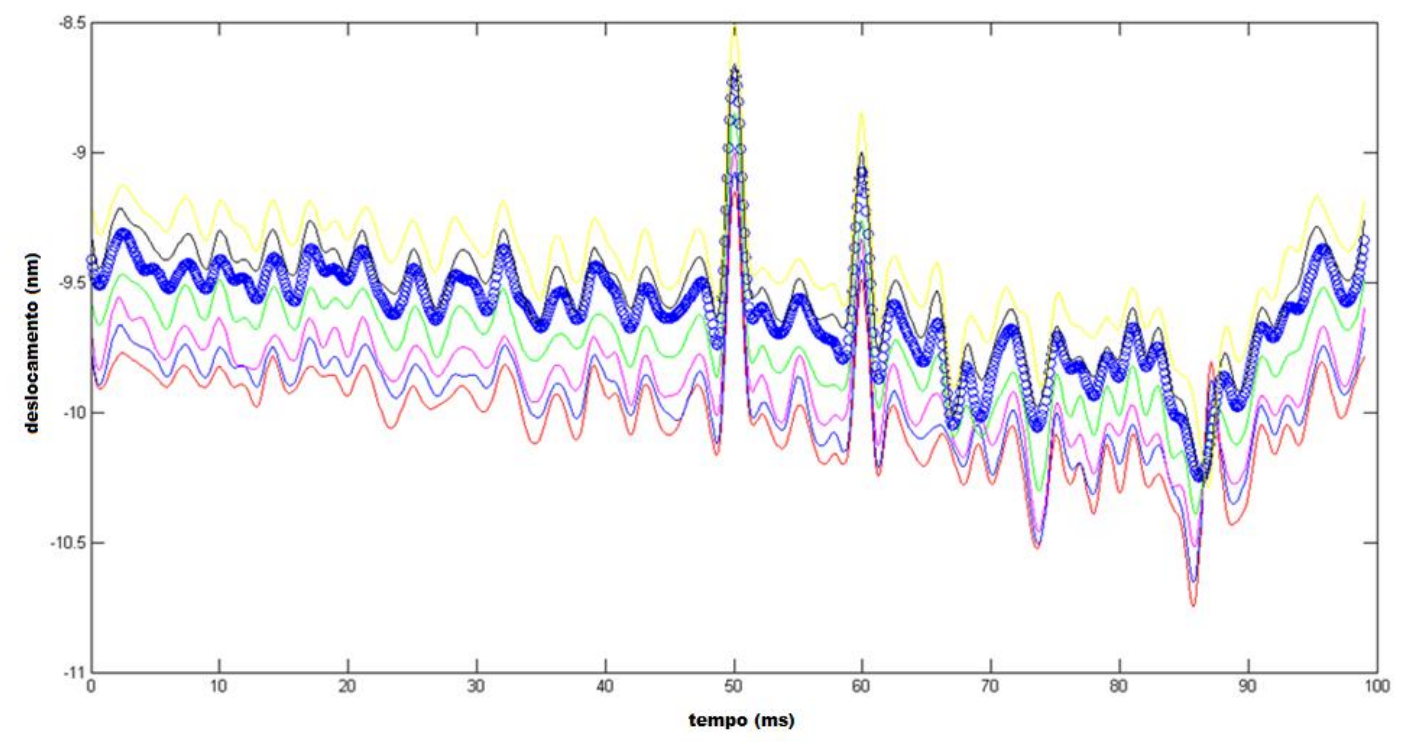

Figura 5. 7 - Relevo da imagem criada pelo modo contato em vermelho e as demais cores em modo não contato.

Os experimentos representadas na Fig 5.8-5.13 são representados no histórico de deslocamento no tempo e foram escaneadas em uma região em linha reta, sendo representados os deslocamentos pelo tempo (histórico) para diferentes valores de $\mathrm{z}$ no modo não contato.

A figura 5.8 mostra a microviga em uma altura de $671.32 \mathrm{~nm}$ com o intuito de observar as deflexões que geram as imagens.

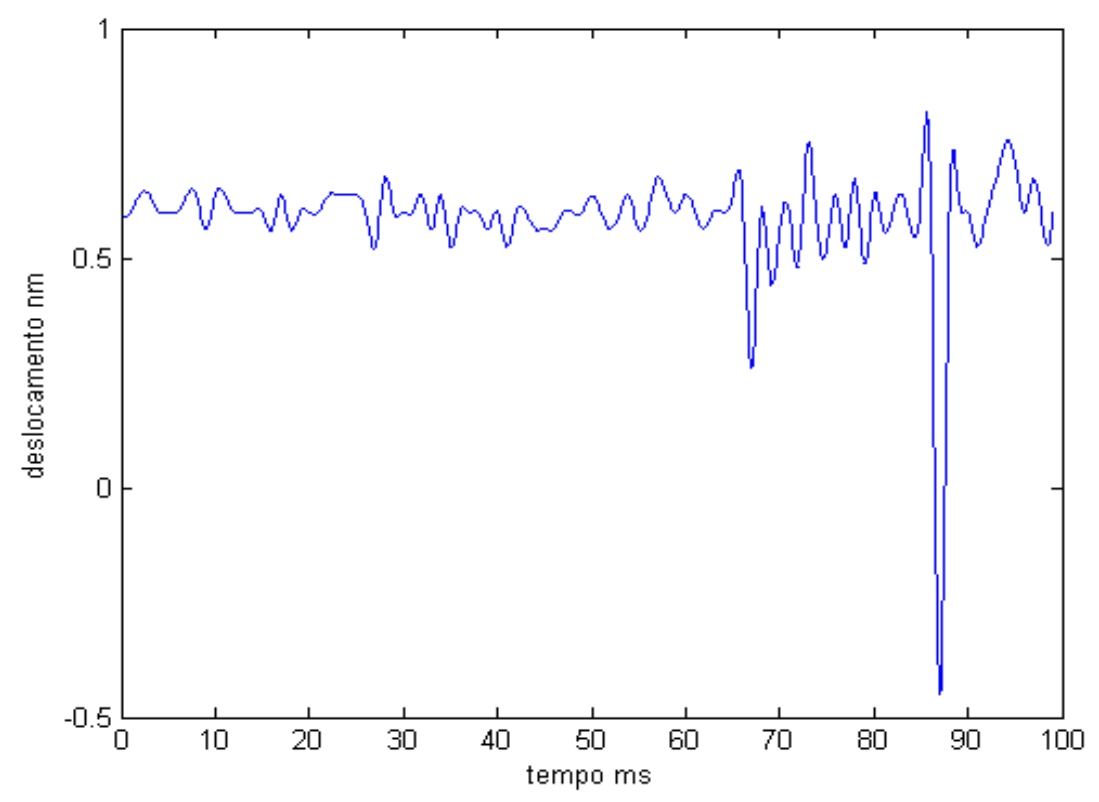

Figura $5.8-z=671.32 \mathrm{~nm}$ 


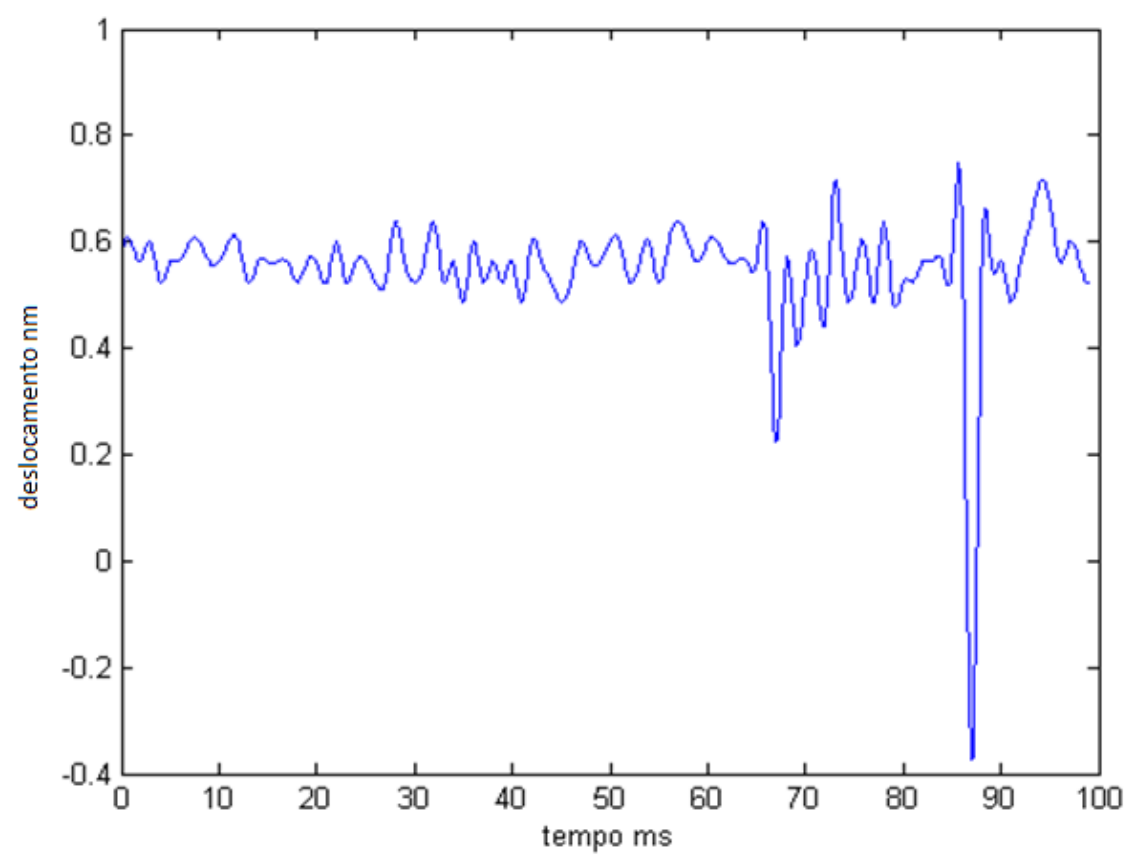

Figura $5.9-z=634.65 \mathrm{~nm}$

A série temporal da Fig. 5.9 tem uma altura de escaneamento um pouco menor do que a apresentada em Fig. 5.8 e mostra grande semelhança gráfica.

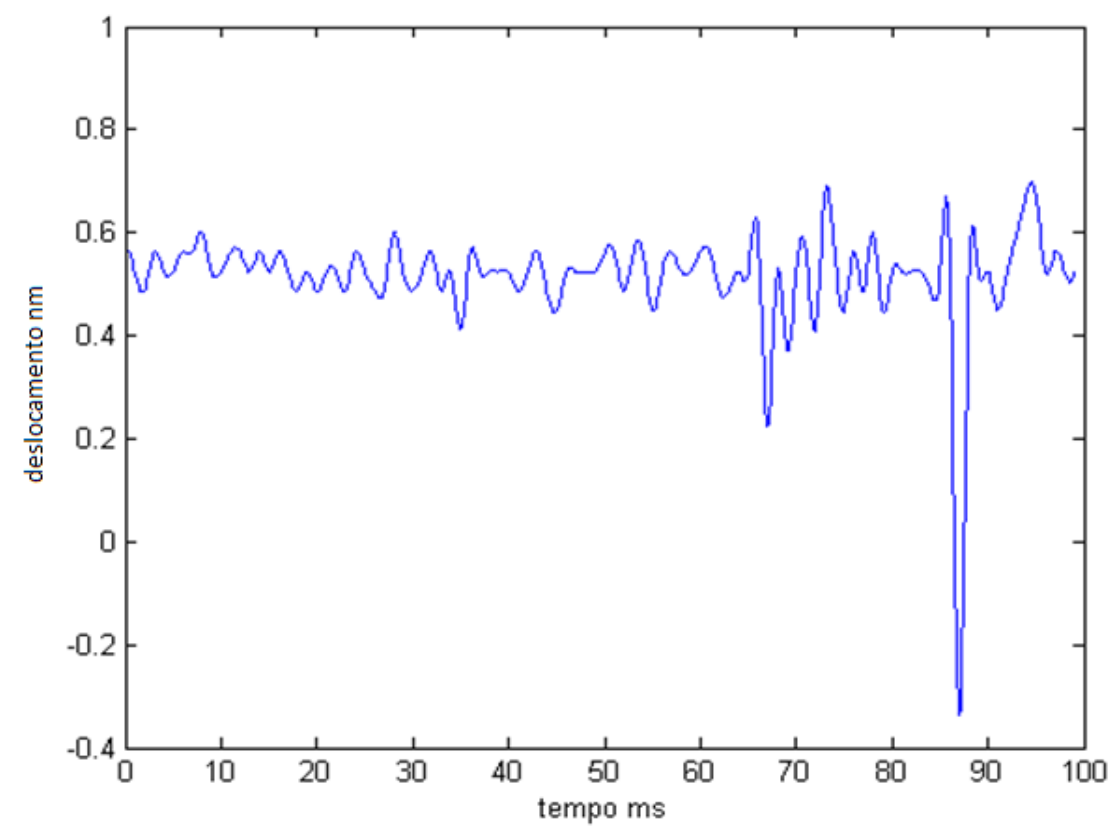

Figura $5.10-z=592.34 \mathrm{~nm}$

A Fig. 5.10 ainda mantém as características da Fig. 5.9 com o pico de deslocamento em aproximadamente $87 \mathrm{~ms}$. 


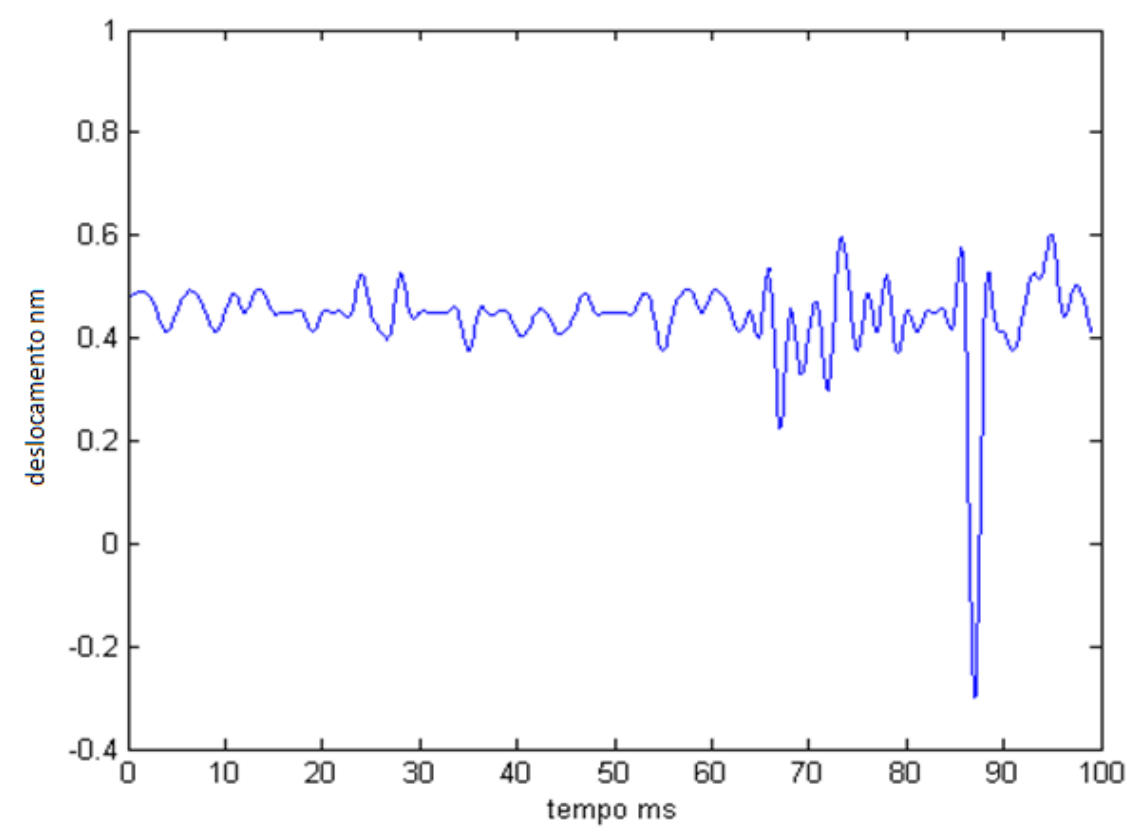

Figura $5.11-z=507.64 \mathrm{~nm}$

A Fig. 5.11 mostra os deslocamentos do microviga em uma altura já mais reduzida e mantêm a característica das séries anteriores.

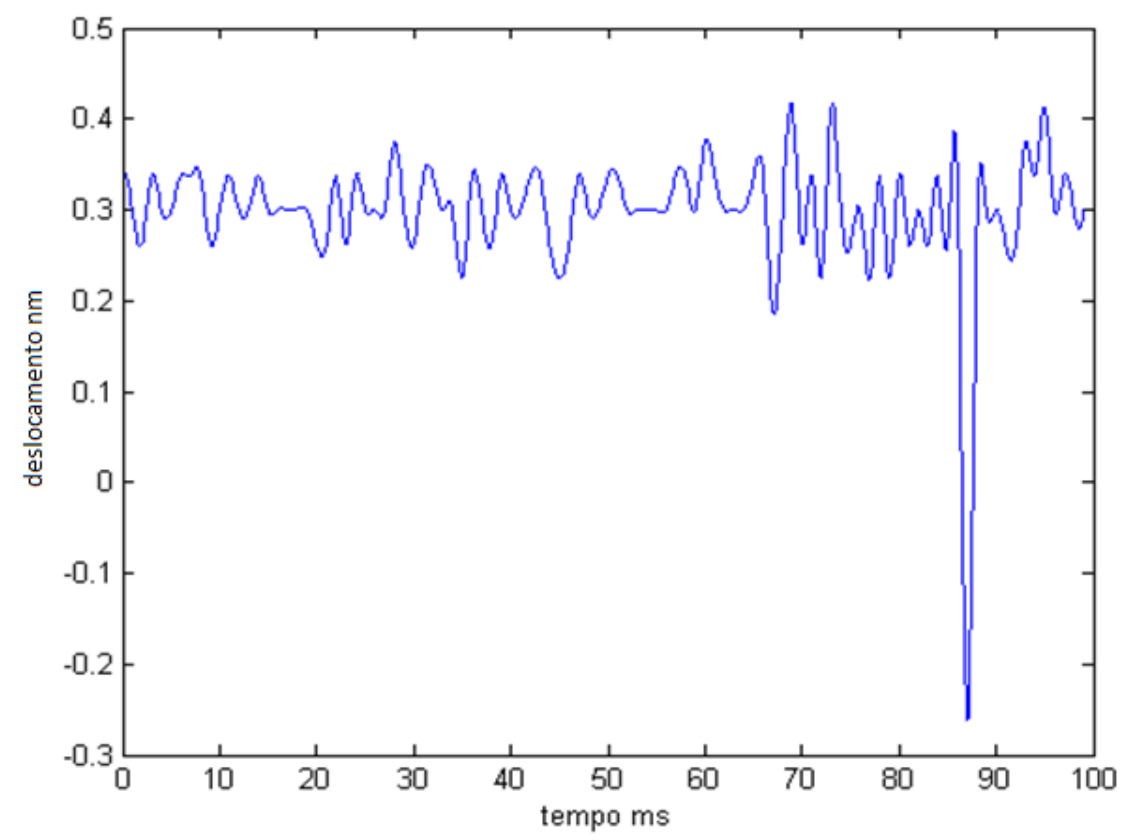

Figura $5.12-\mathrm{z}=338.24 \mathrm{~nm}$

A série temporal da Fig. 5.12 mantêm a característica das séries anteriores com uma maior suavidade nas curvas do intervalo de tempo entre 0 e $50 \mathrm{~ms}$. 


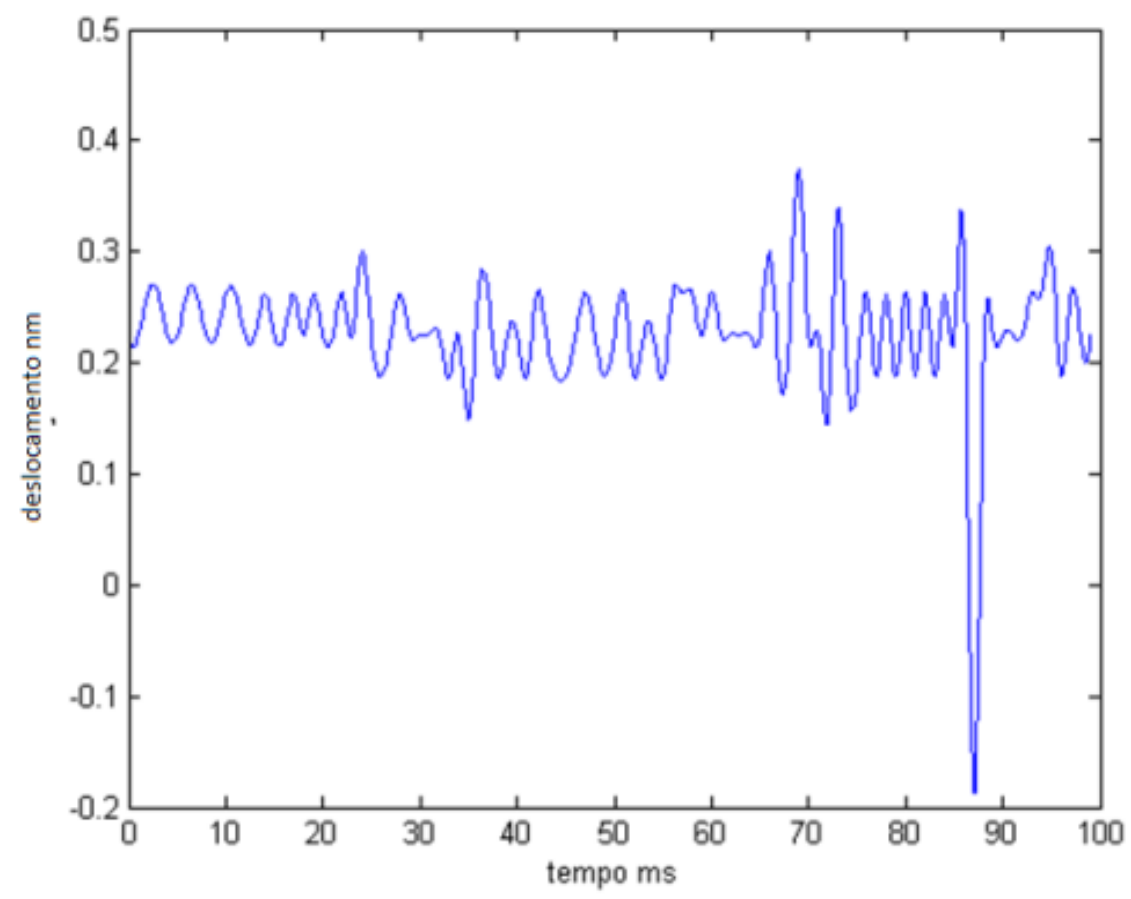

Figura $5.13-z=253.86 \mathrm{~nm}$

$\mathrm{Na}$ Fig. 5.13 a série temporal de altura $\mathrm{z}=253.86 \mathrm{~nm}$ mostra que microviga produziu uma série com mais regularidade, incluindo curvas suaves e dentro de uma amplitude mais regular.

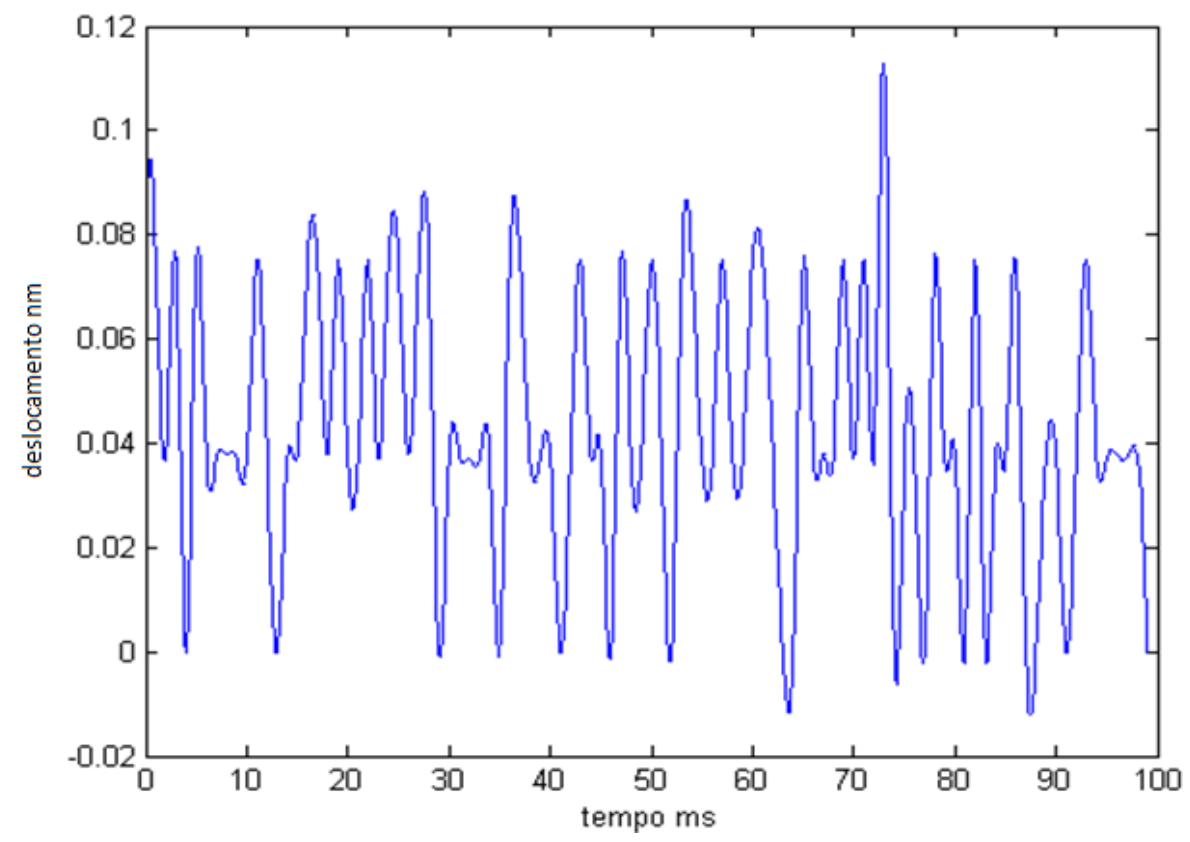

Figura 5. 14- $z=50.24 \mathrm{~nm}$

Na Fig. 5.14 a microviga na altura $\mathrm{z}=50.24 \mathrm{~nm}$ produziu uma série temporal com amplitudes mais definidas e sem o pico de $87 \mathrm{~ms}$. 
Na próxima seção são mostradas séries temporais com a microviga retangular em safira cujas vibrações foram captadas em um único ponto da amostra de safira. A microviga foi fixada em um único ponto, vibrando em diversas alturas pelo modo soft tapping e assim não gerando imagens. Em um único ponto pode-se observar as diferentes deflexões da microviga considerando forças de interação ponta a amostra com valores fixos, isto é, sem variar de ponto a ponto, como no experimento em uma linha reta.

\subsection{SÉRIES TEMPORAIS CONSTRUÍDAS COM DEFLEXÕES EM UM ÚNICO PONTO DA AMOSTRA}

O experimento foi feito com amostra de safira, em um único ponto de vibração, constante elástica da microviga de $42 \mathrm{~N} / \mathrm{m}$, ponteira padrão de formato retangular 'Sens. amp/sens' de $100 \mathrm{~nm} / \mathrm{V}$, amplitude 'setpoint' de $178.85 \mathrm{mV}$, 'drive setpoint' de $48.52 \mathrm{mV}$, e temperatura da sala de $22.1^{\circ} \mathrm{C}$.

O importante é notar que o simples fato de mudar uma única variável (z) na aquisição das séries, muitas diferenças são notadas, como alguns picos de amplitudes e variações de amplitudes. As Figuras 5.15 - 5.24 representam algumas séries temporais obtidas variando a altura da ponteira e amostra.

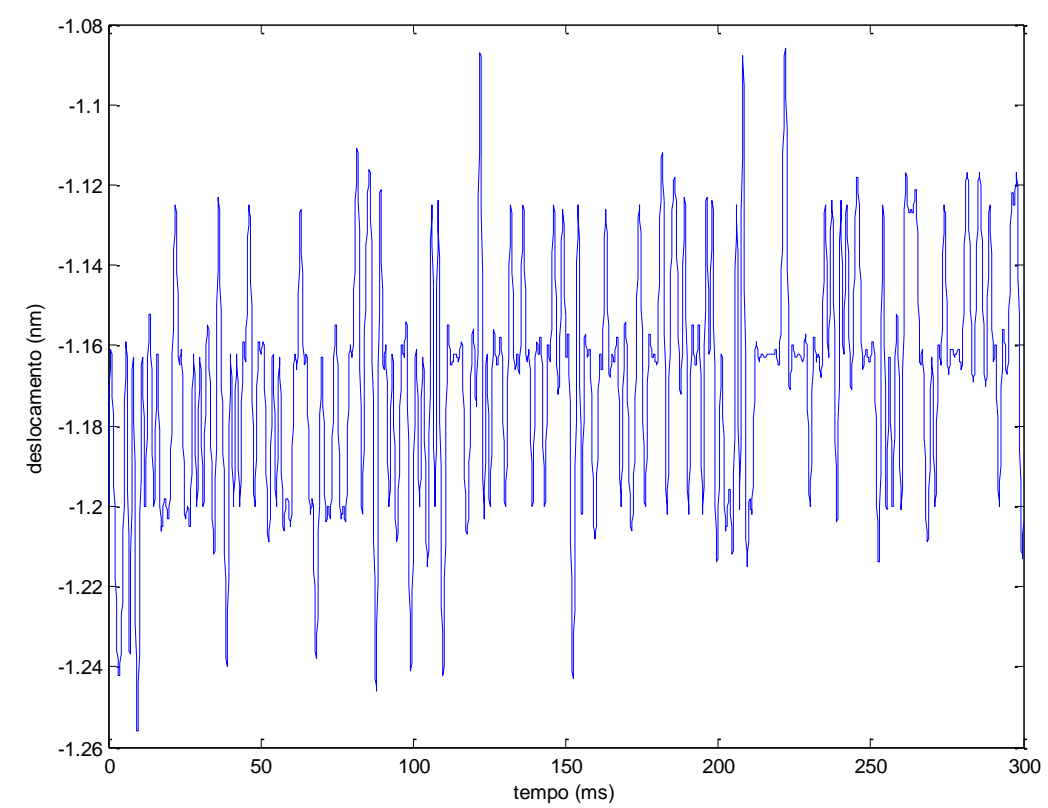

Figura 5. 15 - Histórico de deflexões para $z=622.2 \mathrm{~nm}$ 


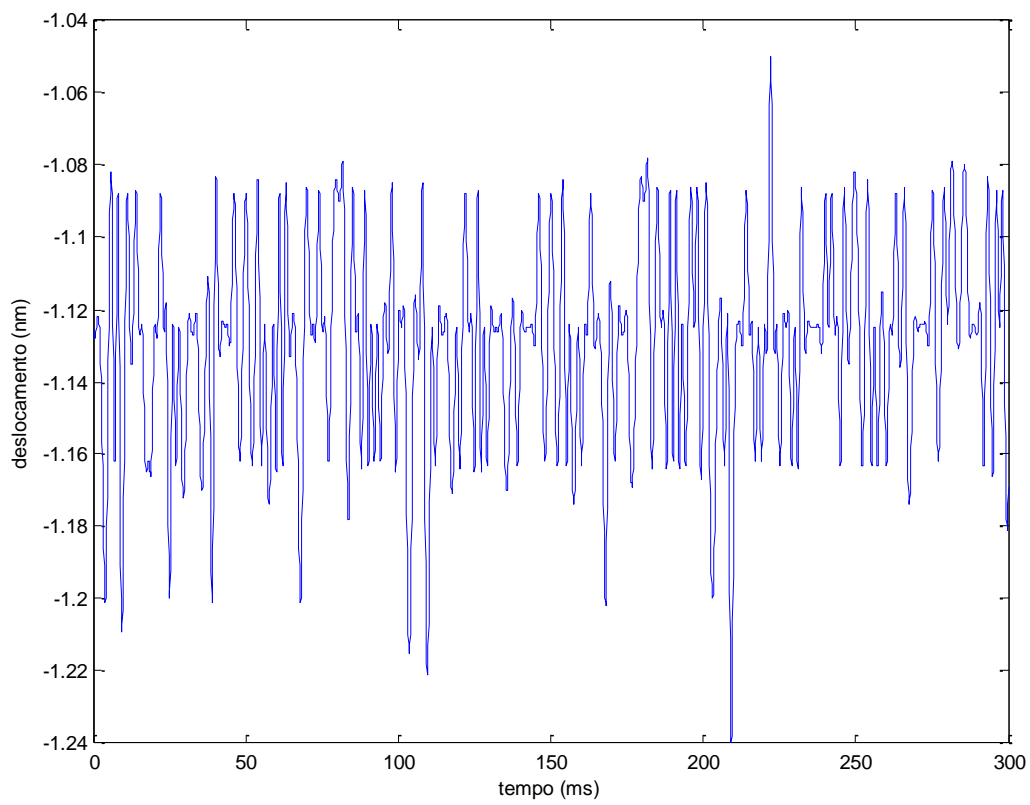

Figura 5. 16 - Histórico de deflexões para $z=605.2 \mathrm{~nm}$

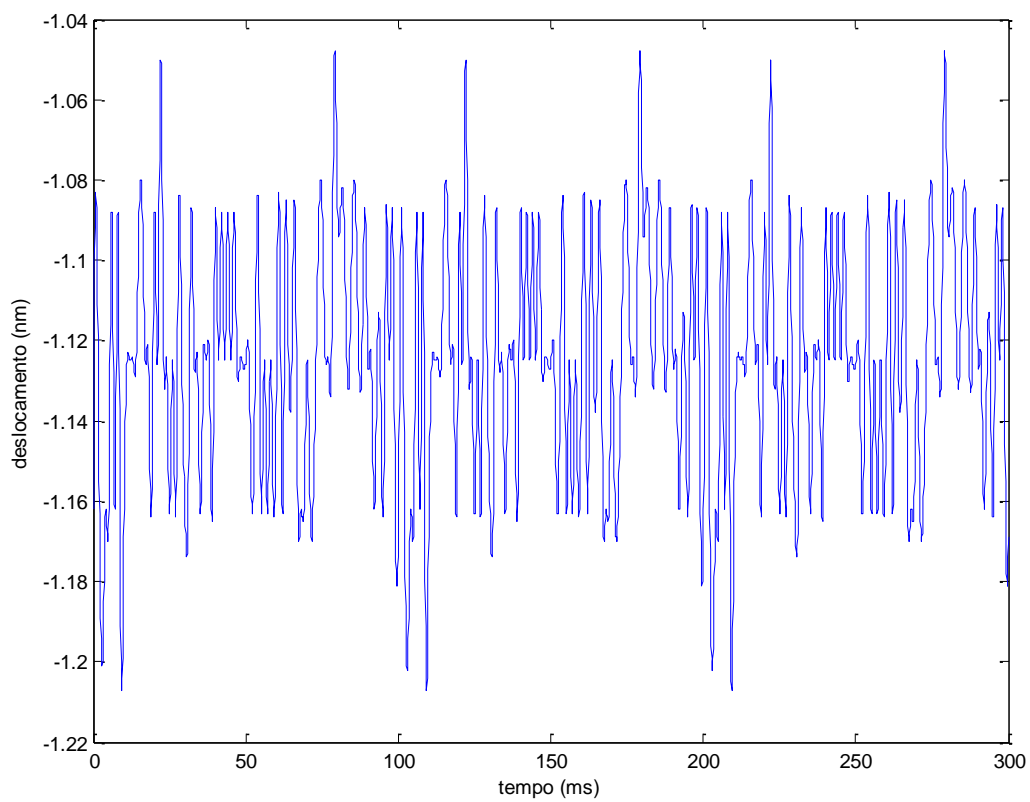

Figura 5. 17 - Histórico de deflexões para $z=588.2 \mathrm{~nm}$ 


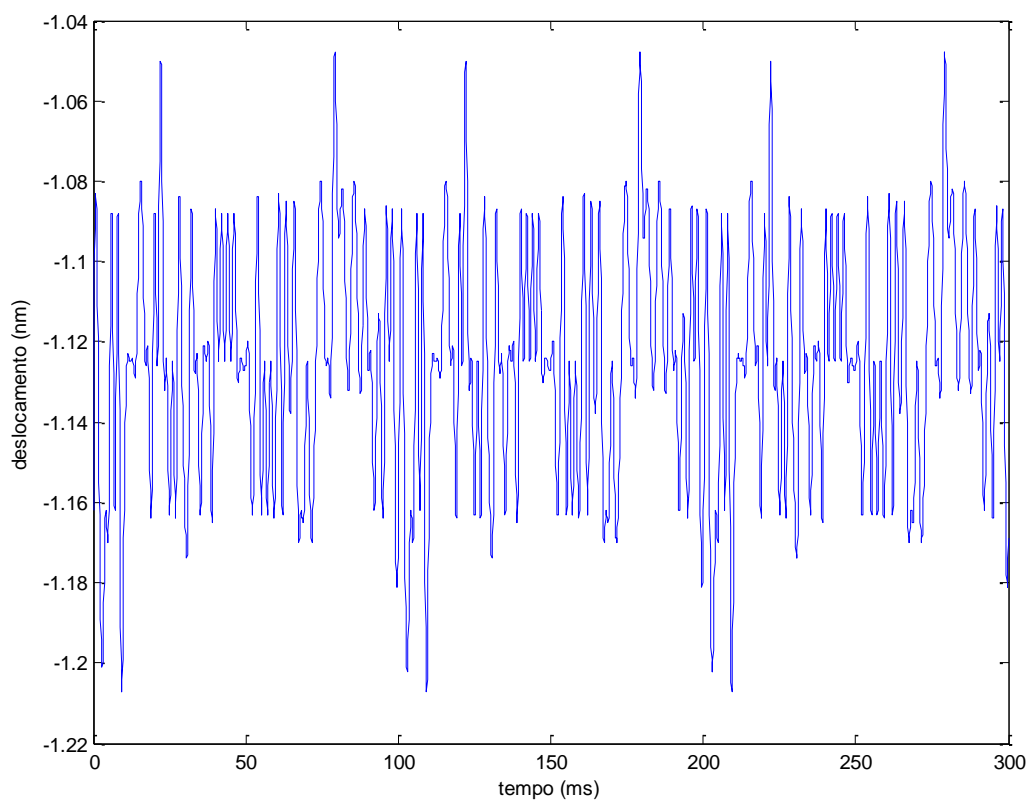

Figura 5. 18 - Histórico de deflexões para $z=571.2 \mathrm{~nm}$

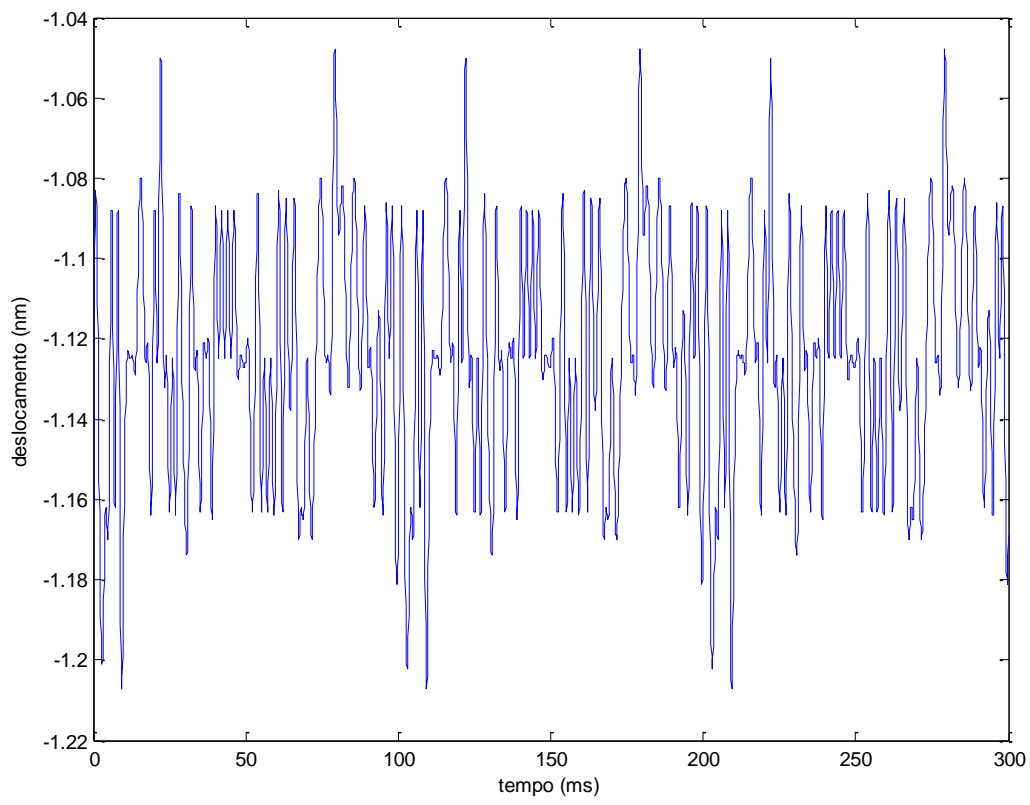

Figura 5. 19- Histórico de deflexões para $z=554.2 \mathrm{~nm}$ 


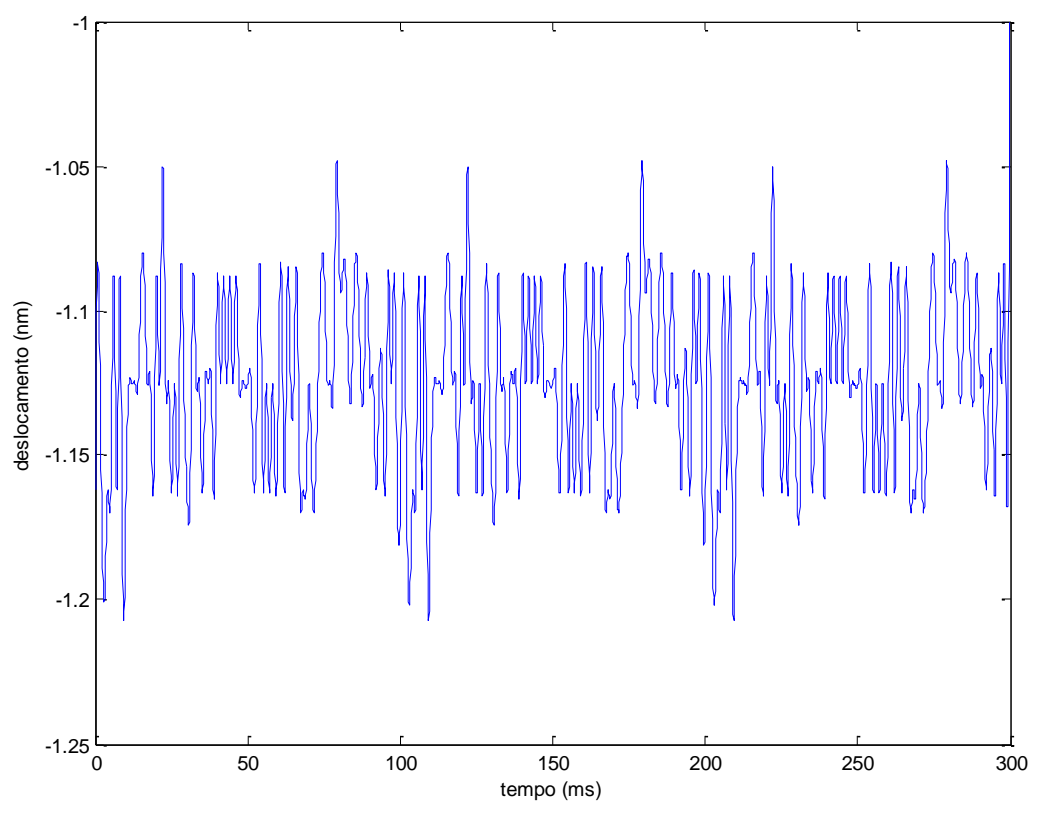

Figura 5. 20 - Histórico de deflexões para $z=537.2 \mathrm{~nm}$

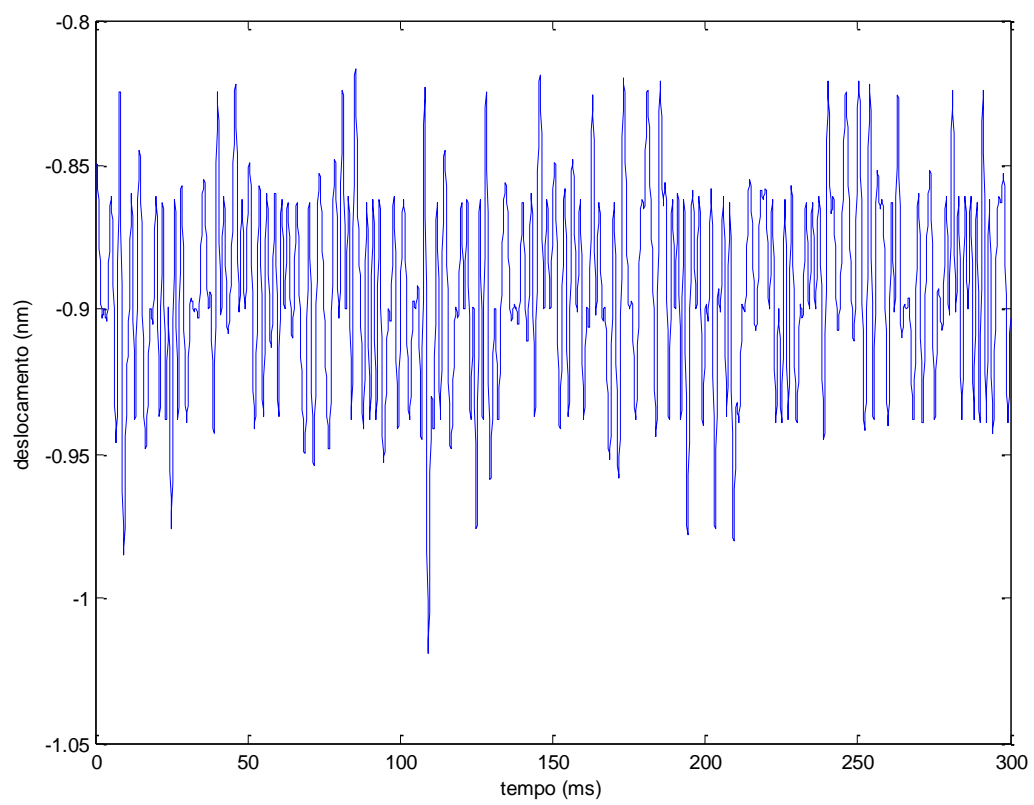

Figura 5. 21- Histórico de deflexões para $z=520.2 \mathrm{~nm}$ 


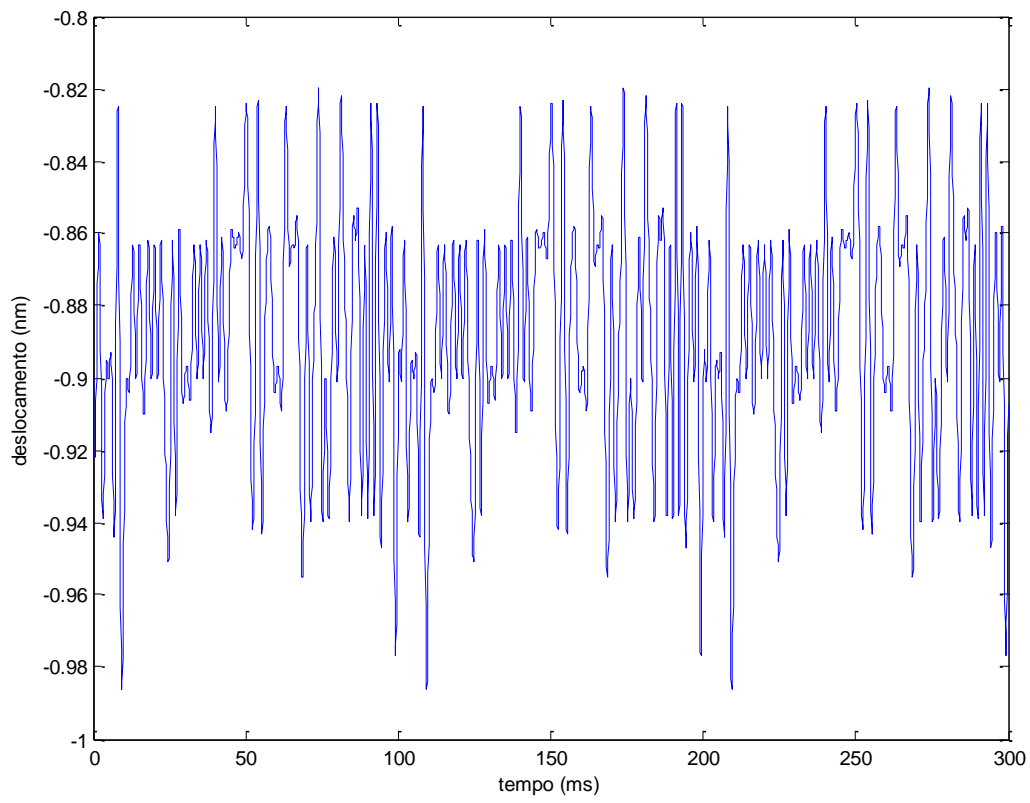

Figura 5. 22- Histórico de deflexões para $z=503.2 \mathrm{~nm}$

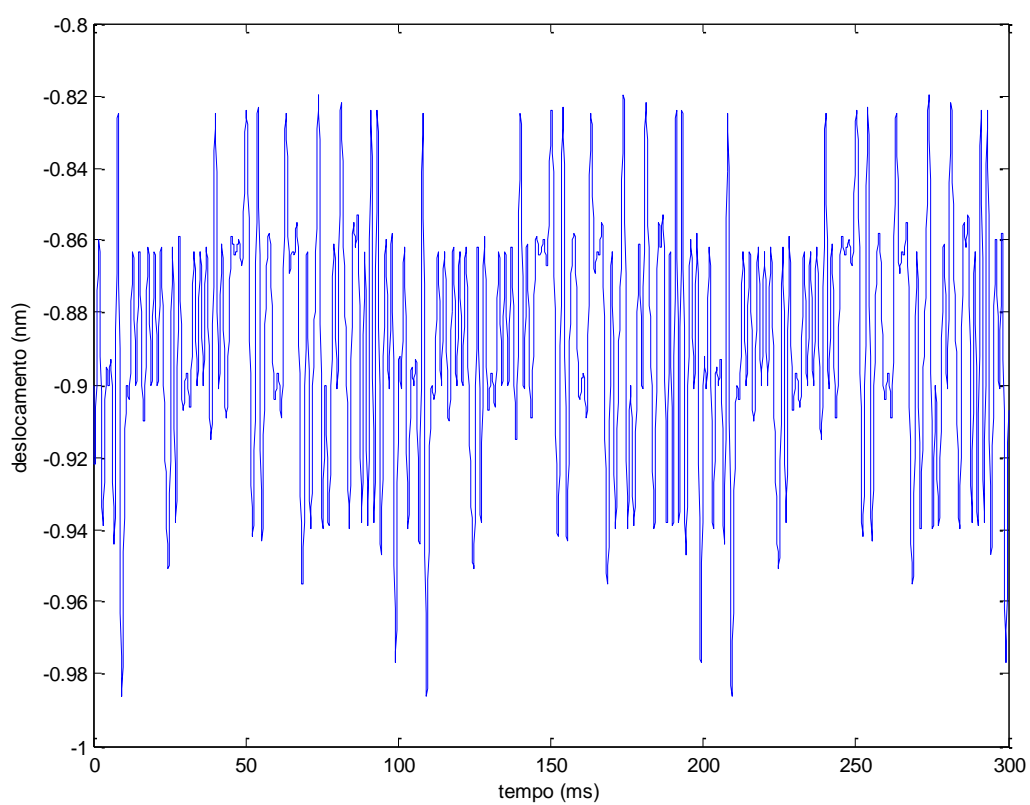

Figura 5. 23- Histórico de deflexões para $z=486.2 \mathrm{~nm}$ 


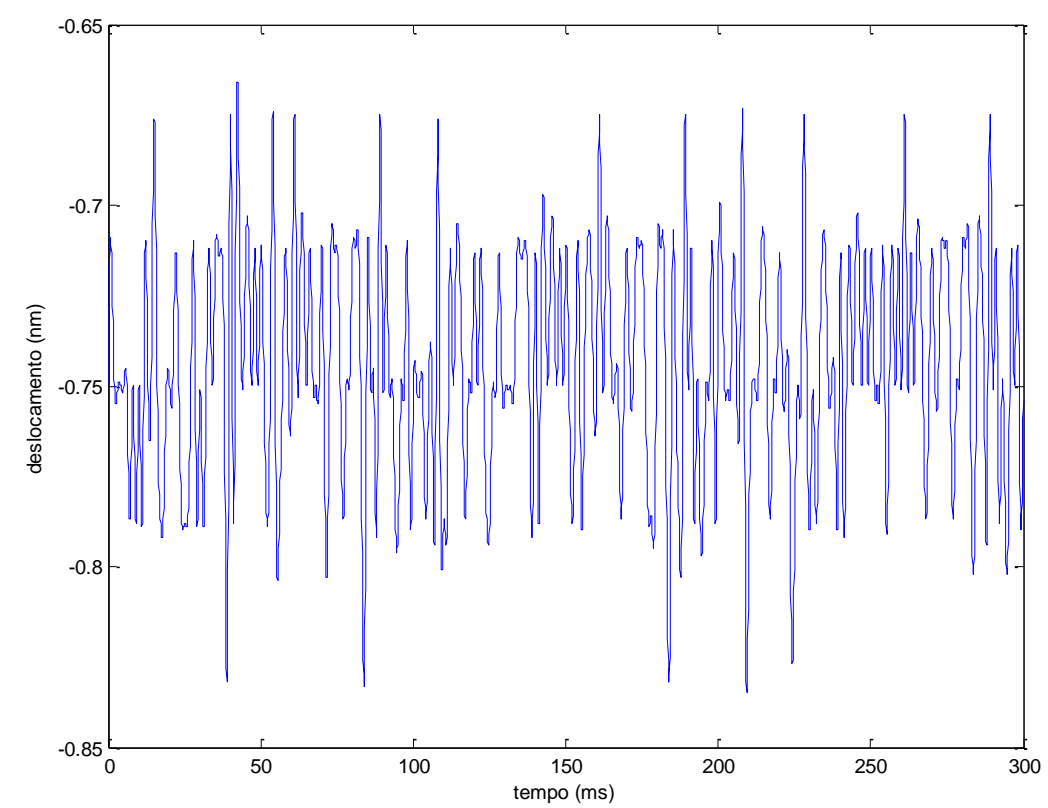

Figura 5. 24 - Histórico de deflexões para $z=401.2 \mathrm{~nm}$

É importante notar que as séries temporais que foram captadas vibrando em um único ponto geram pequenas diferenças gráficas e que essas pequenas diferenças podem ser mensuradas através de testes que medem o quanto elas podem estar próxima de uma dinâmica caótica ou de uma dinâmica periódica. Somente com uma análise utilizando ferramentas como o teste 0-1 e escala indexada, pode se ter essas distinções entre elas mensurando-as em mais ou menos caóticas em um mapa no fim do capítulo do capítulo 5.

\subsubsection{HISTÓRICO NO TEMPO PARA O MODO TAPPING}

Nesta seção são apresentadas os históricos de deflexões em modo 'soft tapping' em um único ponto. $\mathrm{O}$ experimento foi feito com amostra de safira, constante elástica da microviga de $42 \mathrm{~N} / \mathrm{m}$, ponteira padrão de formato retangular 'Sens. amp/sens' de 100nm/ $V$, amplitude 'setpoint' de $178.85 \mathrm{mV}$, 'drive setpoint' de $48.52 \mathrm{mV}$, e temperatura da sala de $22.1^{\circ} \mathrm{C}$. As figuras mostram as séries temporais quando a ponteira movimenta-se vibrando de cima para baixo, isto é, o modo 'soft tapping' em descida variando do seu ponto $\mathrm{z}$ mais alto $(622 \mathrm{~nm}$ ponta-amostra) até a o momento em que encosta na amostra $(\mathrm{z}=0 \mathrm{~nm})$. Nas Figs. 5.25 - 5.33 observa-se a falta de padrão nas séries temporais que foram captadas em sequência. 


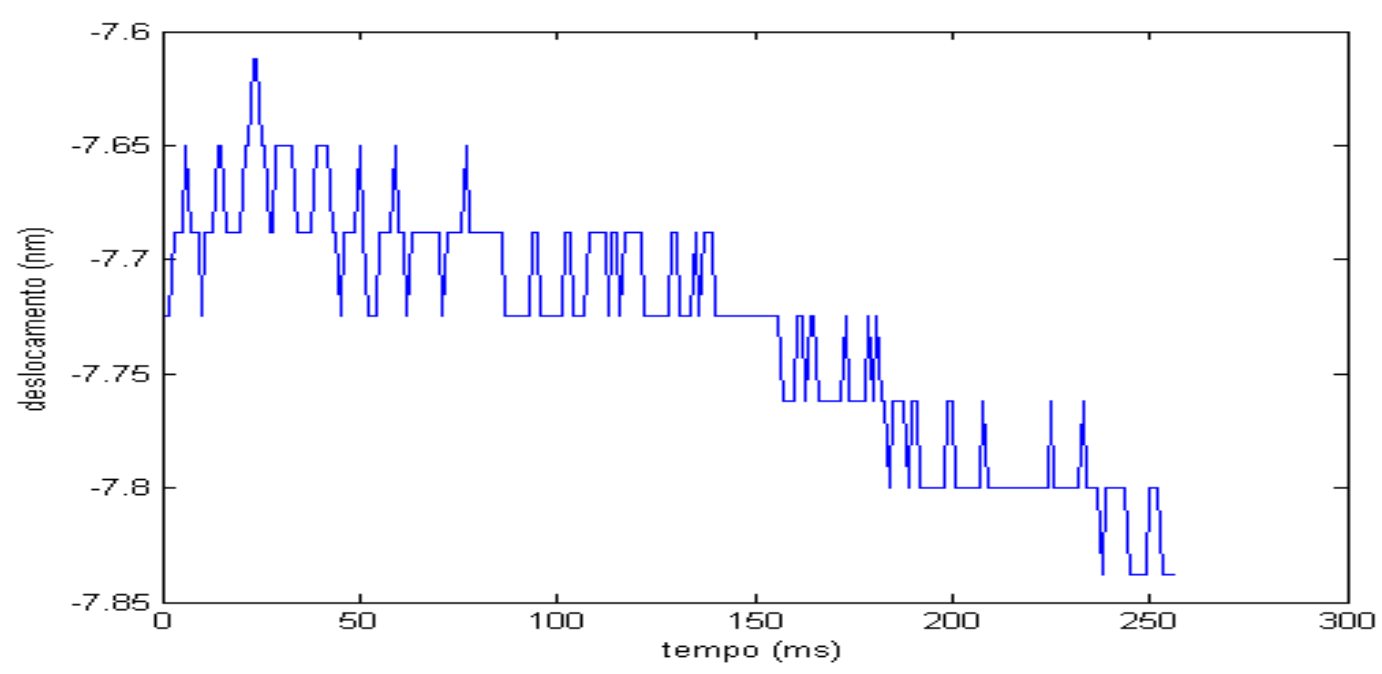

Figura 5. 25 - Série temporal do AFM em descida no modo tapping

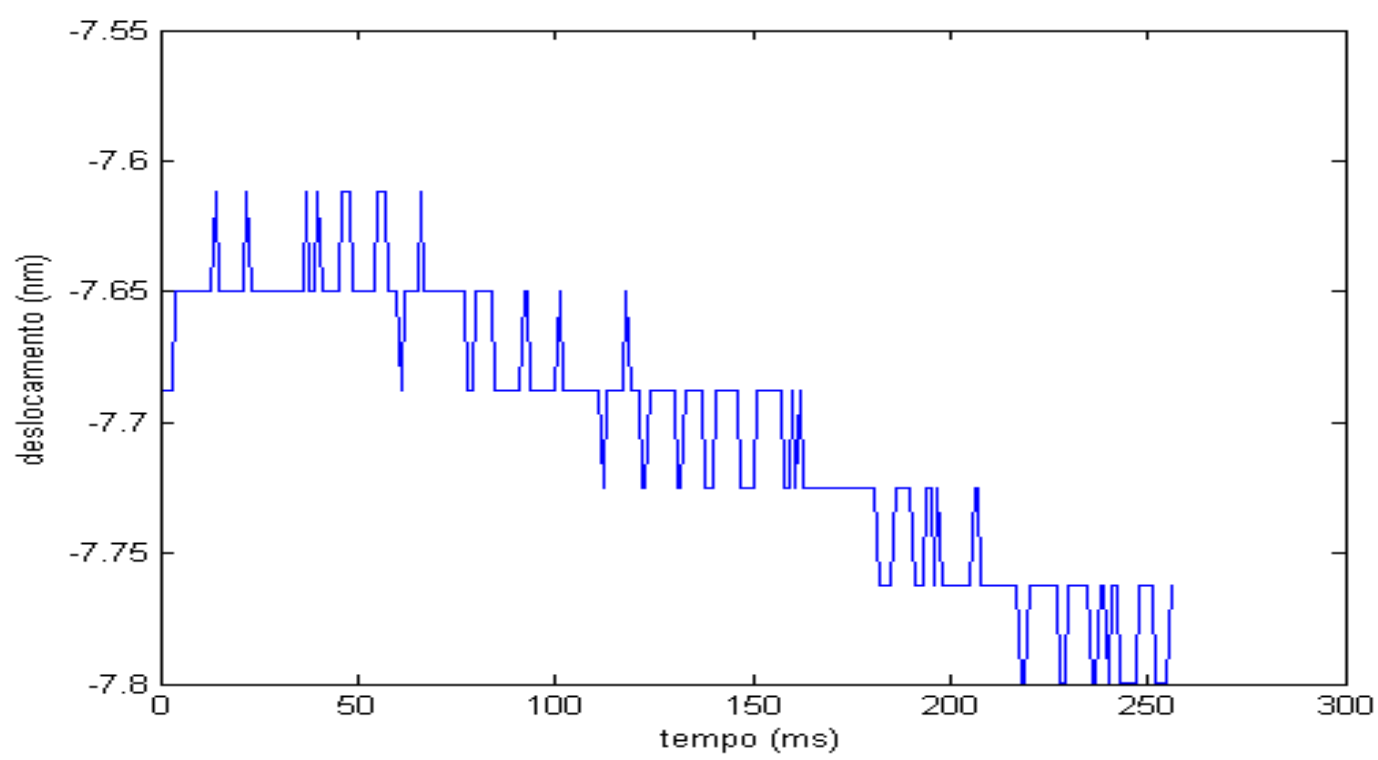

Figura 5. 26 - Série temporal do AFM em descida no modo tapping 


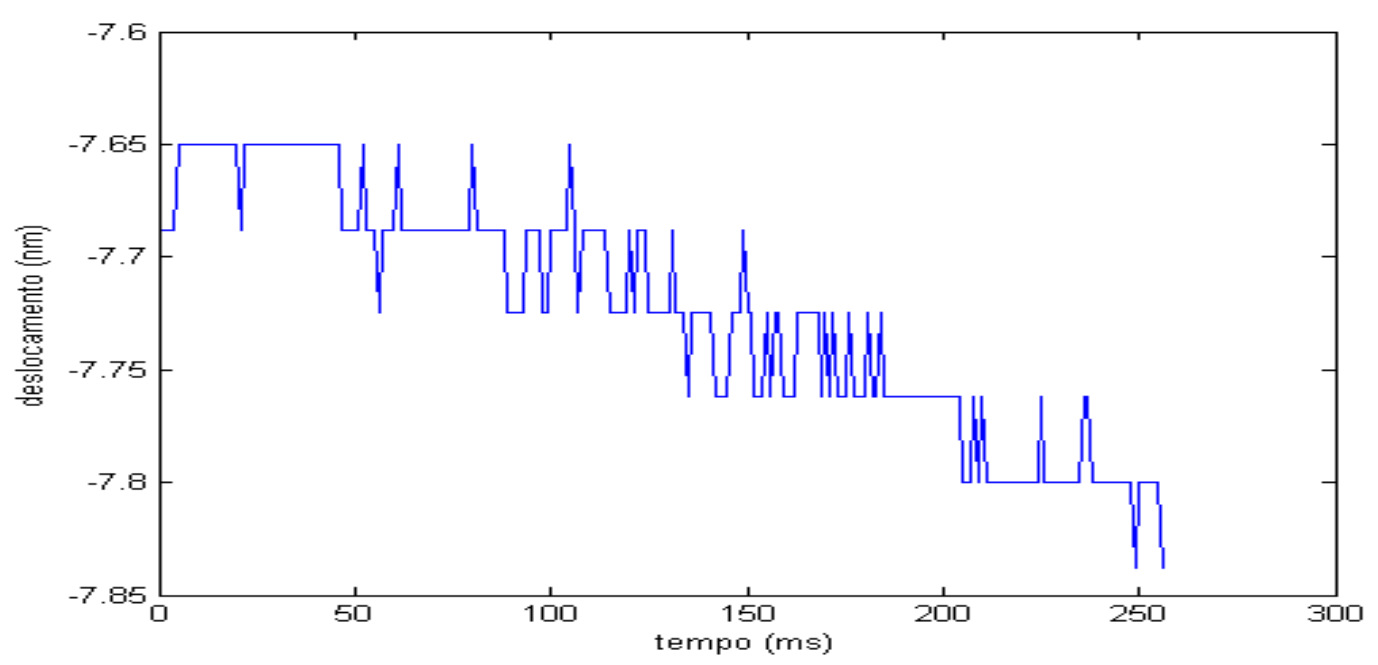

Figura 5. 27- Série temporal do AFM em descida no modo tapping

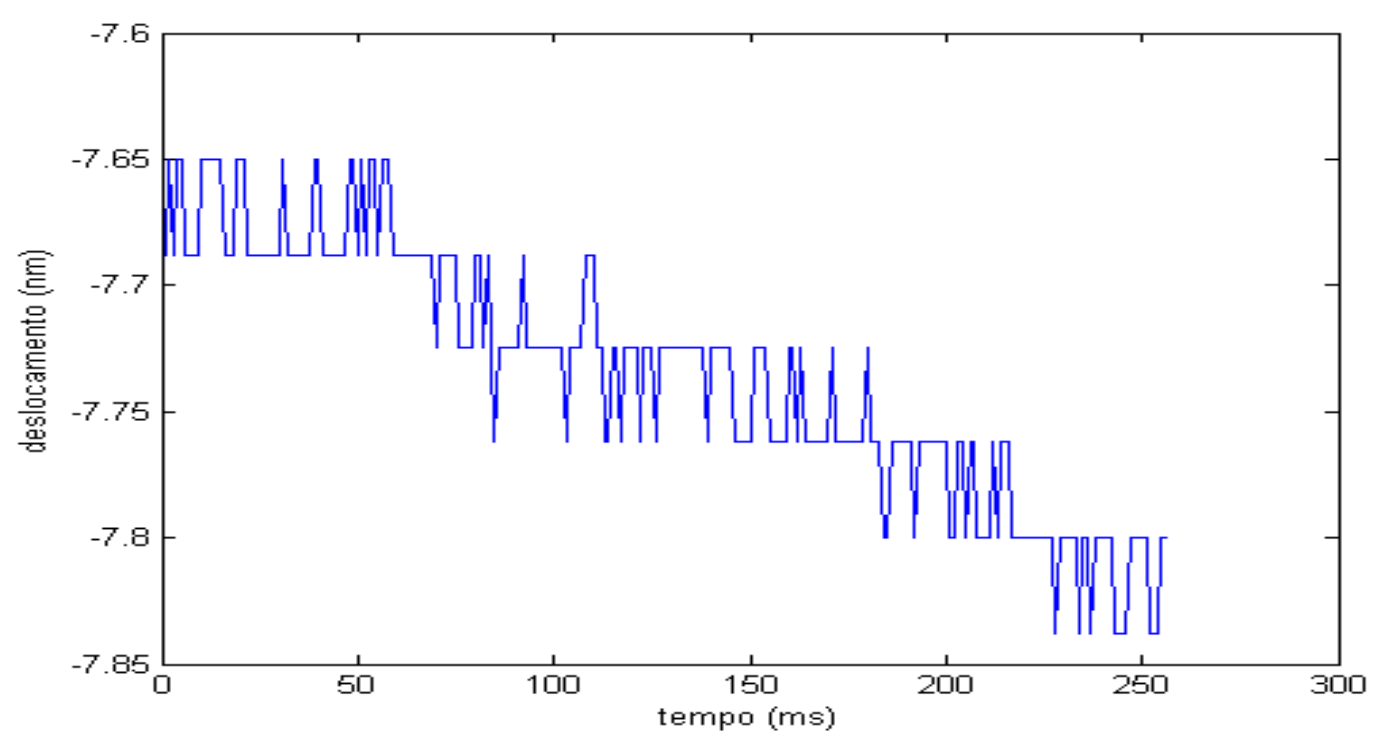

Figura 5. 28 - Série temporal do AFM em descida no modo tapping

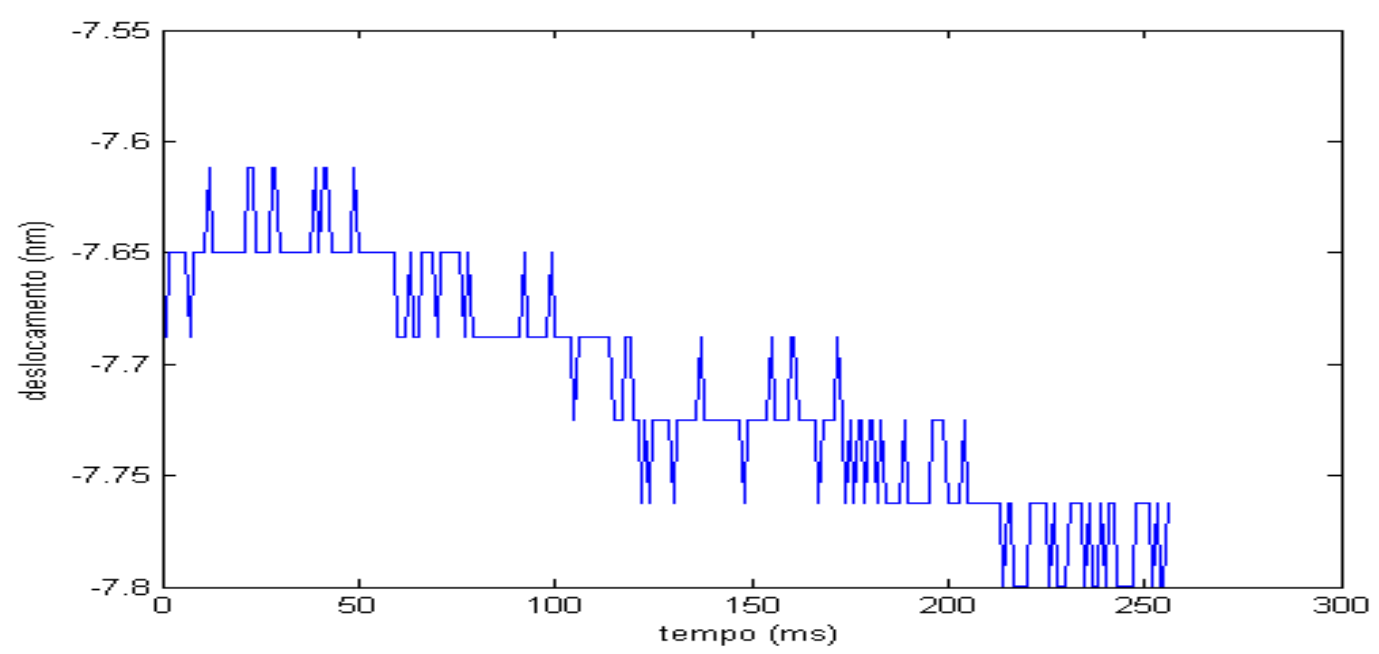

Figura 5. 29- Série temporal do AFM em descida no modo tapping 


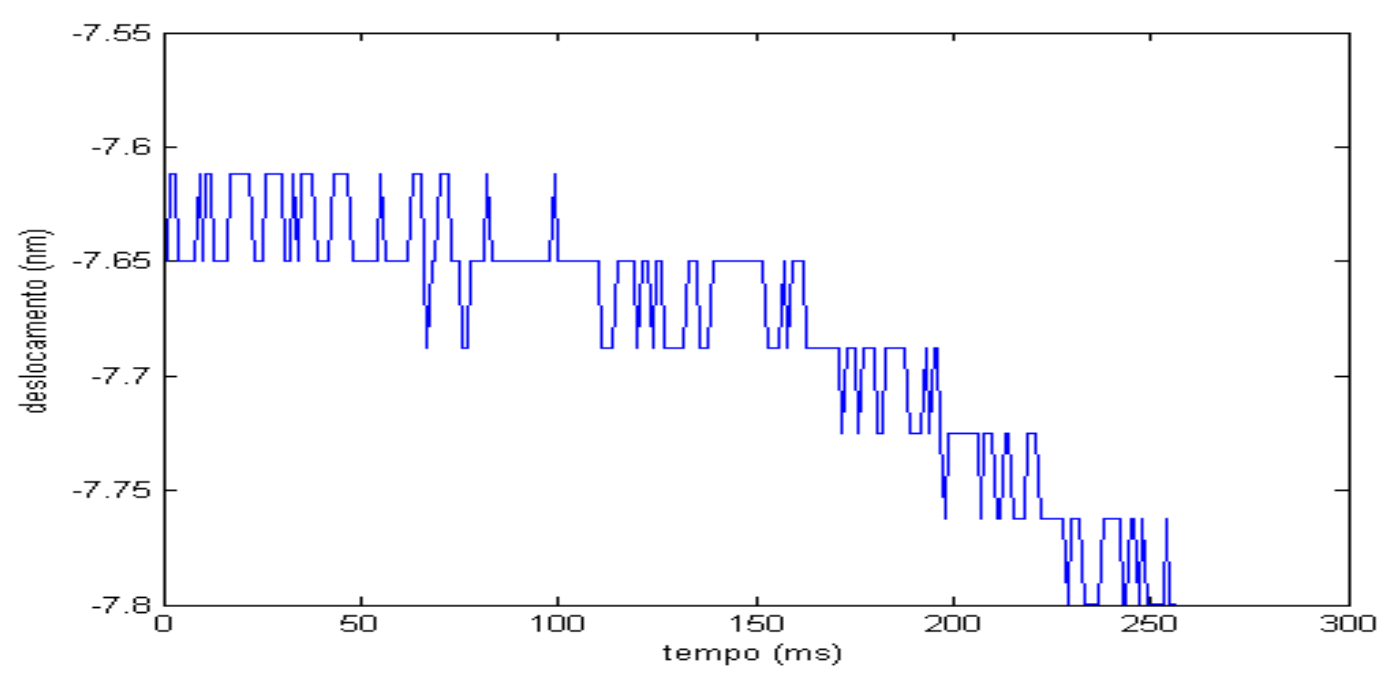

Figura 5. 30 - Série temporal do AFM em descida no modo tapping

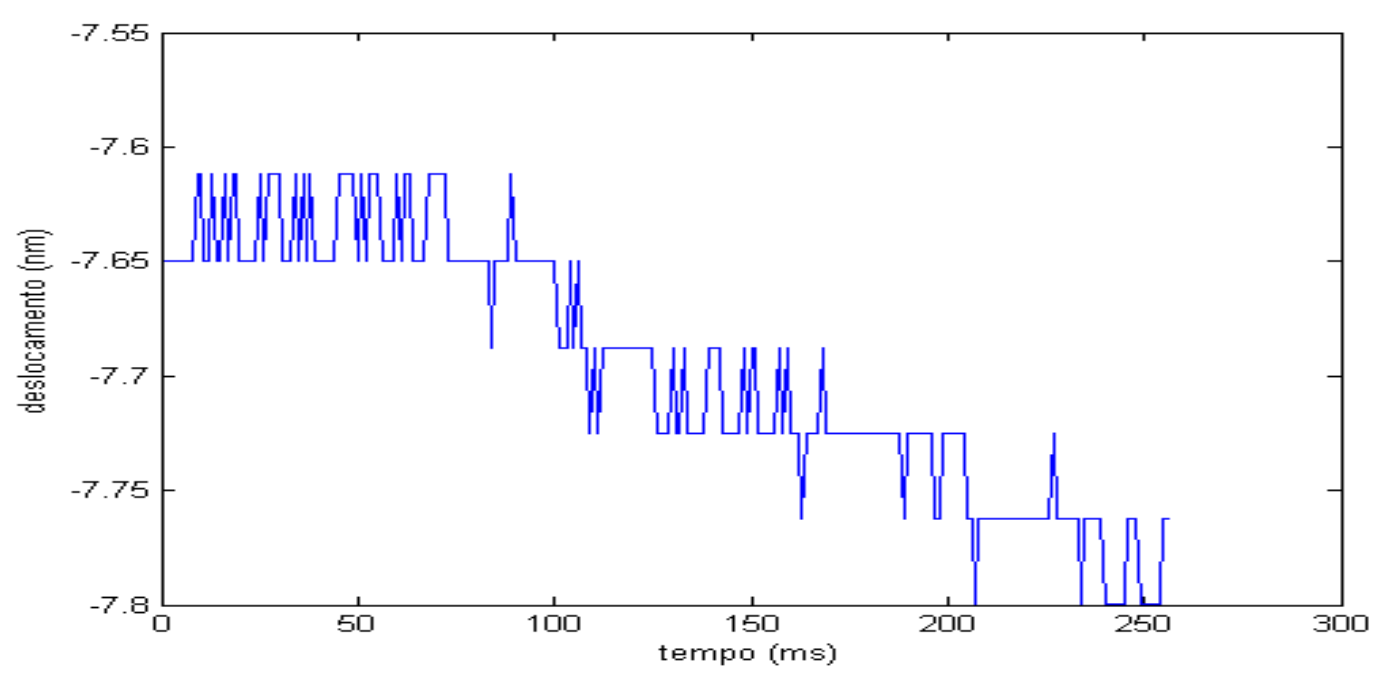

Figura 5. 31- Série temporal do AFM em descida no modo tapping

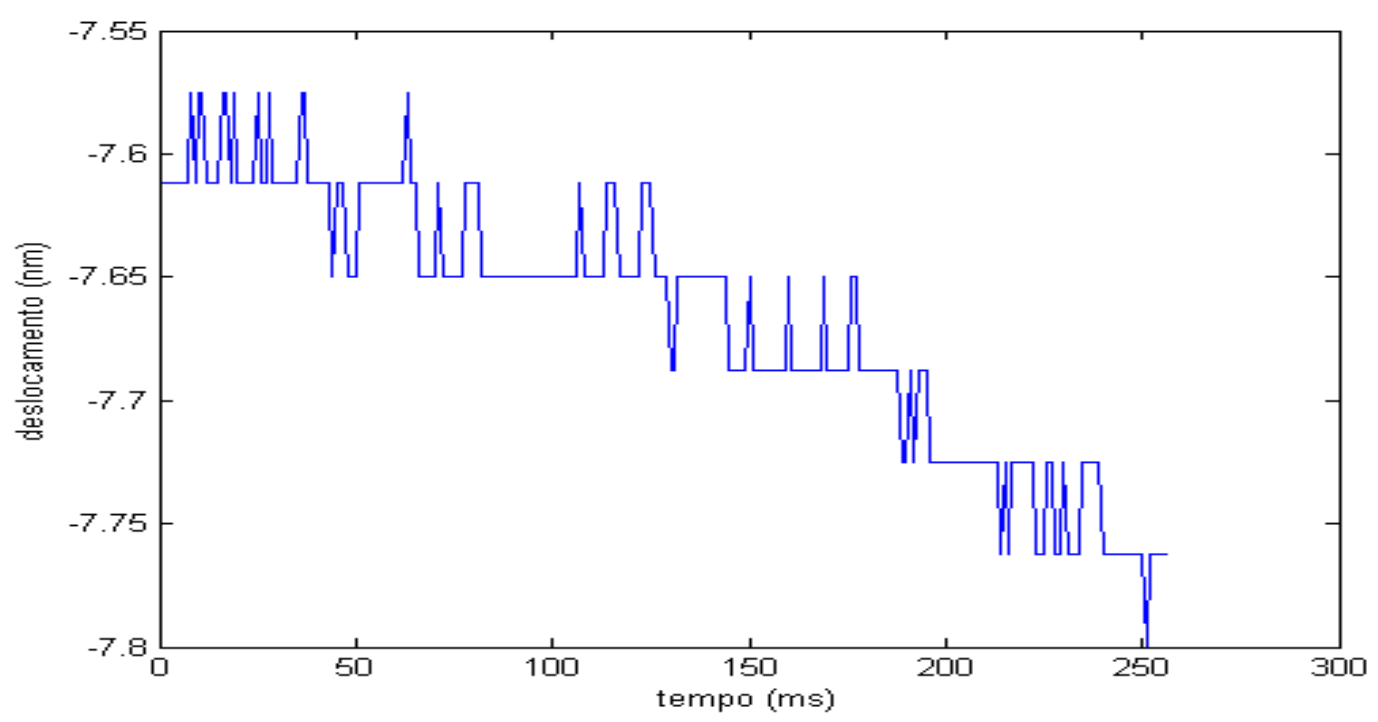

Figura 5. 32- Série temporal do AFM em descida no modo tapping 


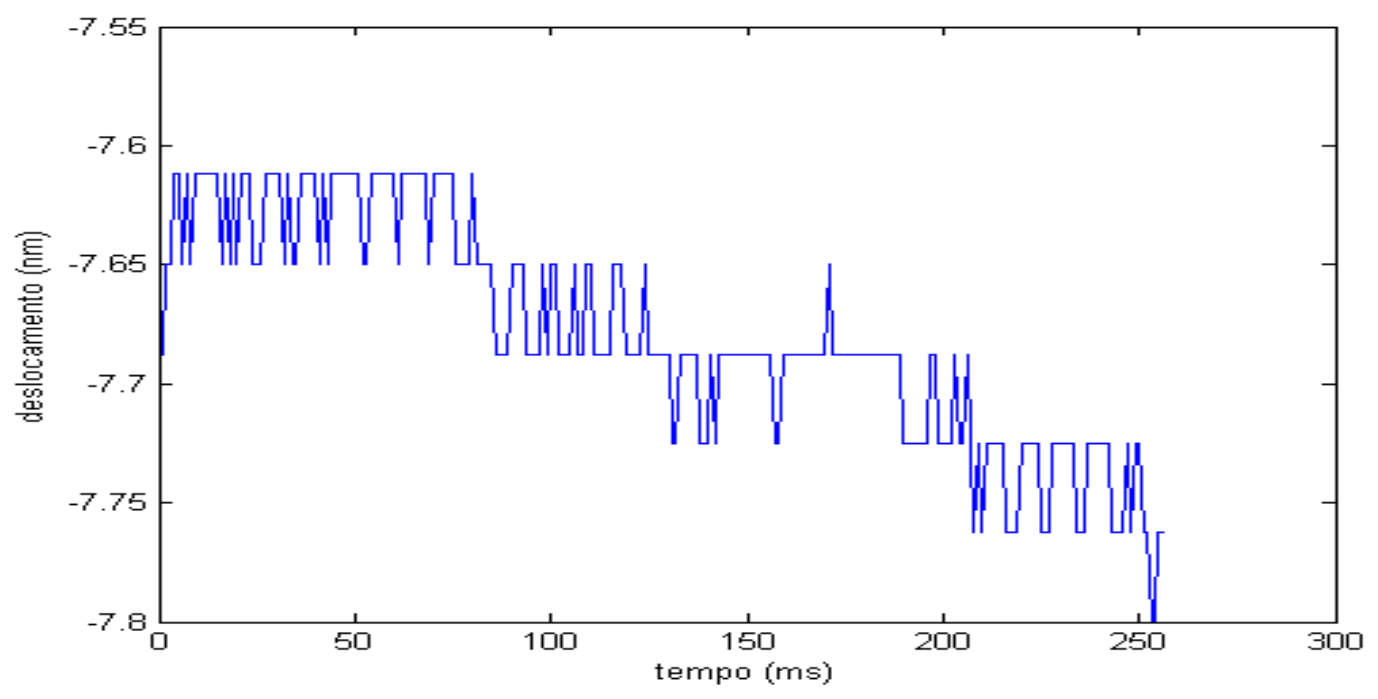

Figura 5. 33- Série temporal do AFM em descida no modo tapping

Percebe-se que em modo tapping é um misto de não contato em diversas alturas. Em cada altura $\mathrm{z}$ a microviga vibra muitas milhões de vezes.

\subsection{ANÁLISE DOS RESULTADOS}

Os arquivos de dados adquiridos representam 37 ou 35 séries temporais de deflexões da microviga com 3000 pontos em modo não contato, resultando em cerca de 110000 pontos. Quatro tipos de experiências foram realizadas e analisadas pelo teste $0-1$ e escala indexada. Para o maior e menor valor do teste 0 - 1 , foram feitas reconstruções do espaço de estados e, finalmente, a FFT da série. $U(t)$ é a nova série com defasagem tempo (atraso $\tau$ ) através do cálculo da informação mútua e dimensão de imersão através do método dos falsos vizinhos próximos.

\subsubsection{SÉRIE TEMPORAL A}

A série temporal 'A' foi captada com a amostra de safira, 'scan size' no valor zero, isto é, não produziu imagem e a fazendo com que a microviga vibrasse em apenas um ponto, e consequentemente fazendo com que as forças de interação entre ponta e amostra não variassem, constante elástica da microviga de $42 \mathrm{~N} / \mathrm{m}$, ponteira padrão retangular (standard), Sens. amp/sens de $100 \mathrm{~nm} / \mathrm{V}$, amplitude setpoint de $178.85 \mathrm{mV}$, drive setpoint de 
$48.52 \mathrm{mV}$ e temperatura do laboratório de $22.1^{\circ} \mathrm{C}$. A tabela 1 indica os valores da teste $0-1 \mathrm{e}$ escala indexada para cada altura $\mathrm{z}$ em que a série foi produzida.

Tabela 2- Tabela com teste 0-1 e escala indexada da série ' $a$ '

\begin{tabular}{c|c|c|c|c|c|c|c|c}
\hline $\mathbf{Z}$ & $\mathbf{K}$ & $\begin{array}{c}\text { LACA } \\
\text { INDE } \\
\text { XADA }\end{array}$ & $\mathbf{Z}$ & $\mathbf{K}$ & $\begin{array}{c}\text { LA } \\
\text { INDE } \\
\text { XADA }\end{array}$ & $\mathbf{Z}$ & $\mathbf{K}$ & $\begin{array}{c}\text { ESCA } \\
\text { LA } \\
\text { INDE } \\
\text { XADA }\end{array}$ \\
\hline 10.2 & 0,99778 & 0,23578 & 231,2 & 0,99236 & 0,99959 & 452,2 & 0,99401 & 0,99964 \\
27,2 & 0,99581 & 0,48769 & 248,2 & 0,99485 & 0,99959 & 469,2 & 0,99372 & 0,99948 \\
44,2 & 0,99716 & 0,50316 & 265,2 & 0,99277 & 0,99959 & 486,2 & 0,9921 & 0,99948 \\
61,2 & 0,99741 & 0,61466 & 282,2 & 0,99528 & 0,99959 & 503,2 & 0,99356 & 0,99948 \\
78,2 & 0,99699 & 0,87823 & 299,2 & 0,99589 & 0,99959 & 520,2 & 0,99348 & 0,99948 \\
95,2 & 0,99775 & 0,67883 & 316,2 & 0,99179 & 0,99959 & 537,2 & 0,99312 & 0,99948 \\
112,2 & 0,99718 & 0,99016 & 333,2 & 0,99318 & 0,99959 & 554,2 & 0,99539 & 0,99948 \\
129,2 & 0,99526 & 0,94879 & 350,2 & 0,99368 & 0,99959 & 571,2 & 0,99442 & 0,99948 \\
146,2 & 0,99325 & 0,99959 & 367,2 & 0,99359 & 0,99959 & 588,2 & 0,99388 & 0,99948 \\
163,2 & 0,99383 & 0,99959 & 384,2 & 0,99262 & 0,99959 & 605,2 & 0,99331 & 0,99948 \\
180,2 & 0,99309 & 0,99959 & 401,2 & 0,99429 & 0,99976 & 622,2 & 0,99395 & 0,99951 \\
197,2 & 0,99333 & 0,99959 & 418,2 & 0,99641 & 0,99972 & & & \\
214,2 & 0,99364 & 0,99959 & 435,2 & 0,99766 & 0,99963 & & & \\
\hline
\end{tabular}

$\mathrm{Na}$ figura 5.34 as séries temporais com as diversas alturas têm seus valores k's indicados no gráfico. A altura $\mathrm{z}=10.2 \mathrm{~nm}$ indica a altura de vibração da microviga mais próxima a amostra enquanto $\mathrm{z}=622.2 \mathrm{~nm}$ indica a maior distância entre microviga e amostra. A Fig. 5.35 mostra, pelo teste da escala indexada, os valores a serem interpretados como uma medida de grau não periodicidade de sinal: a escala indexada será zero ou muito perto de zero para sequências periódicas e perto de um para sequências altamente não periódicas (BENÍTEZ et al., 2010). 


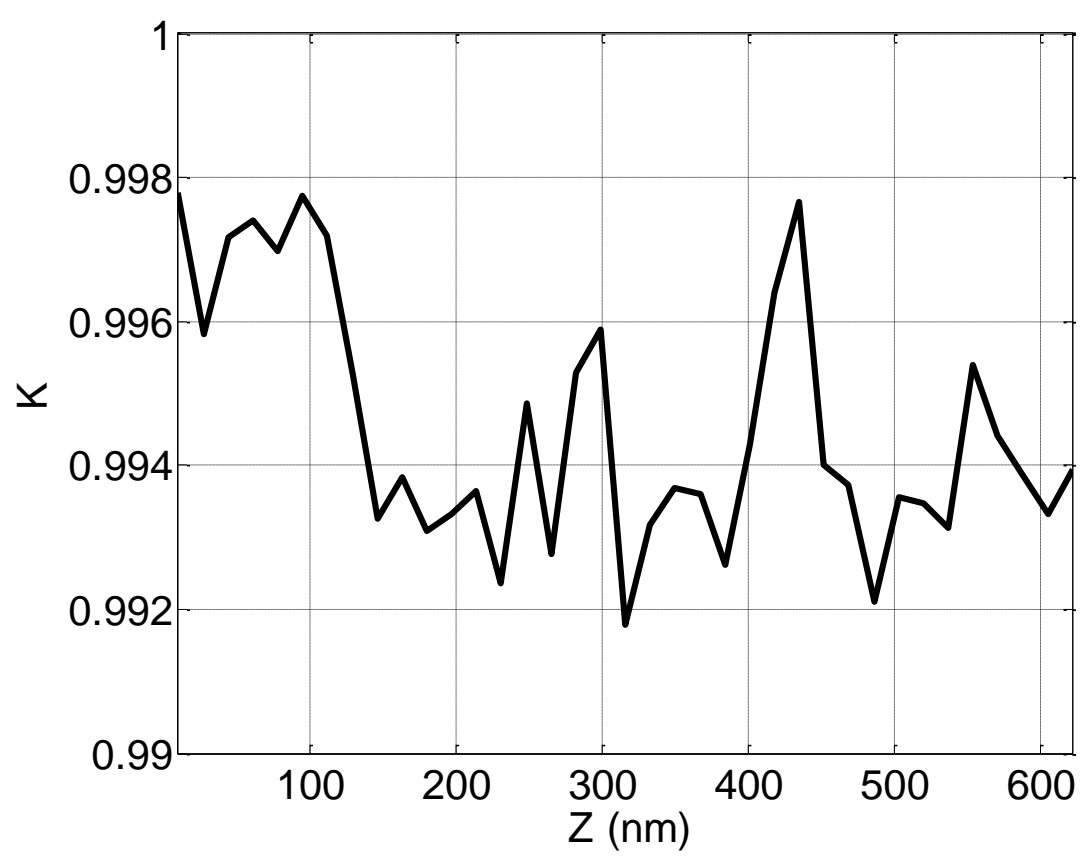

Figura 5. 34 - Gráfico de variação de $K$ para série 'a'

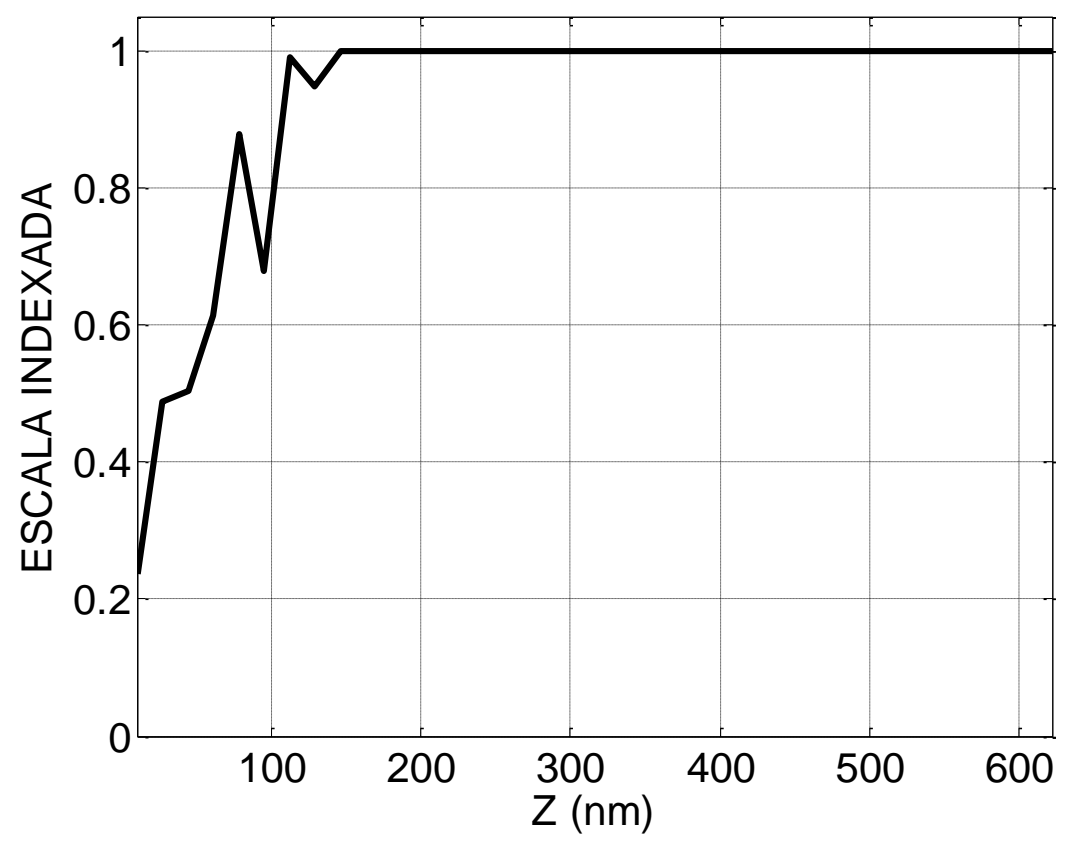

Figura 5. 35 - Escala indexada para série ' $a$ '

Neste experimento ' $A$ ' a série temporal na altura do ponto $Z=316.2 \mathrm{~nm}$ se comporta menos caótica, obtendo o menor valor de k pelo teste $0-1$. Quando $Z=10.2 \mathrm{~nm}$ o teste $0-1$ indica o máximo valor para $K$ e ocorre quando a distância de interação ponta amostra é muito pequena, e consequentemente mostra a microviga sendo afetada com maior intensidade pela força de Van der Waals. O teste da escala indexada mostra um resultado 
diferente ao teste $0-1$. Na posição $\mathrm{z}=10.2 \mathrm{~nm}$ a escala indexada mostra esta série em movimento caótico com o maior valor obtido pelo teste. Nesta posição, calcula-se a informação mútua, falsos vizinhos, para se realizar a construção de espaço de estados, e por último a FFT.

A Informação mútua é uma medida que está relacionada à quantidade de informação que uma variável aleatória de uma série $S_{n}$ contém acerca da variável da série $S_{n+\tau}$. Ou seja, a informação mútua é definida como a quantidade de incerteza que é reduzida em uma dada variável, a partir do conhecimento provido pela outra. A informação mútua em seu primeiro mínimo local é mostra o atraso $\tau$ a ser usada na série reconstruída.

Tabela 3 - informação mútua da série A em z=10.2nm

\begin{tabular}{|c|c|}
\hline$\tau$ & Informação mútua \\
\hline 0 & 2.219262 \\
\hline 1 & 1.411467 \\
\hline 2 & 1.103813 \\
\hline 3 & 0.882336 \\
\hline 4 & 0.7241928 \\
\hline 5 & 0.6102807 \\
\hline 6 & 0.5327023 \\
\hline 7 & 0.4619324 \\
\hline 8 & 0.4138463 \\
\hline 9 & 0.3657782 \\
\hline 10 & 0.3466258 \\
\hline 11 & 0.3140007 \\
\hline 12 & 0.2094535 \\
\hline 13 & 0.2043354 \\
\hline 14 & 0.2617966 \\
\hline 15 & 0.2589961 \\
\hline 16 & 0.1745938 \\
\hline 17 & 0.1298761 \\
\hline 18 & 0.1344594 \\
\hline 19 & 0.1419725 \\
\hline 20 & 0.1554092 \\
\hline
\end{tabular}




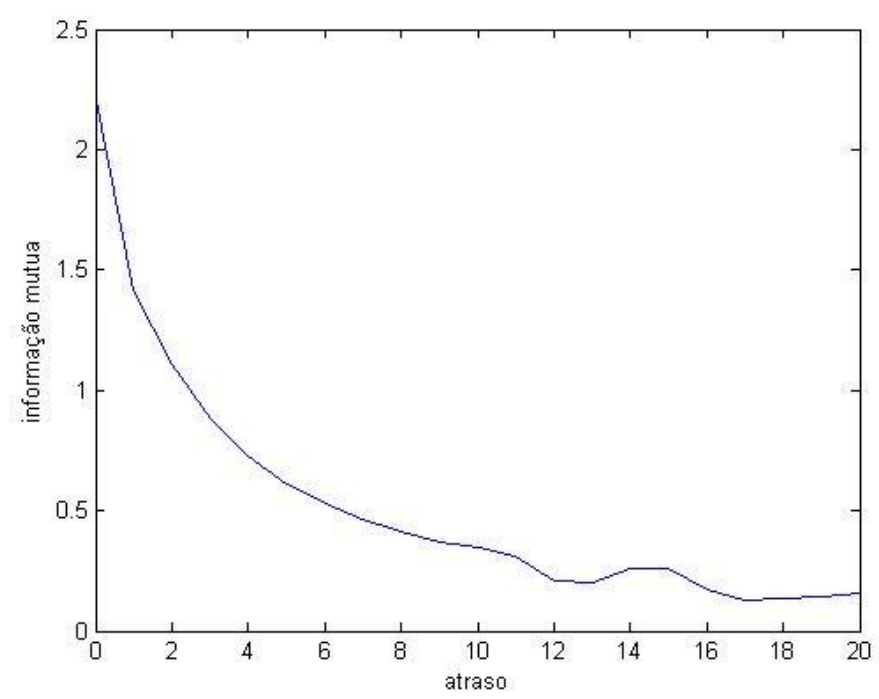

Figura 5. 36 - Informação mútua da série temporal da safira na altura Z=10.2 nm com mínimo local em $\tau=13$

A Fig. 5.36 indica o uso do valor 13 para o atraso, que corresponde ao mínimo local da função. O cálculo dos falsos vizinhos identifica a dimensão em que os termos da série estão inseridos. Na figura 5.37 quando a dimensão é 5 a série não tem falsos vizinhos próximos.

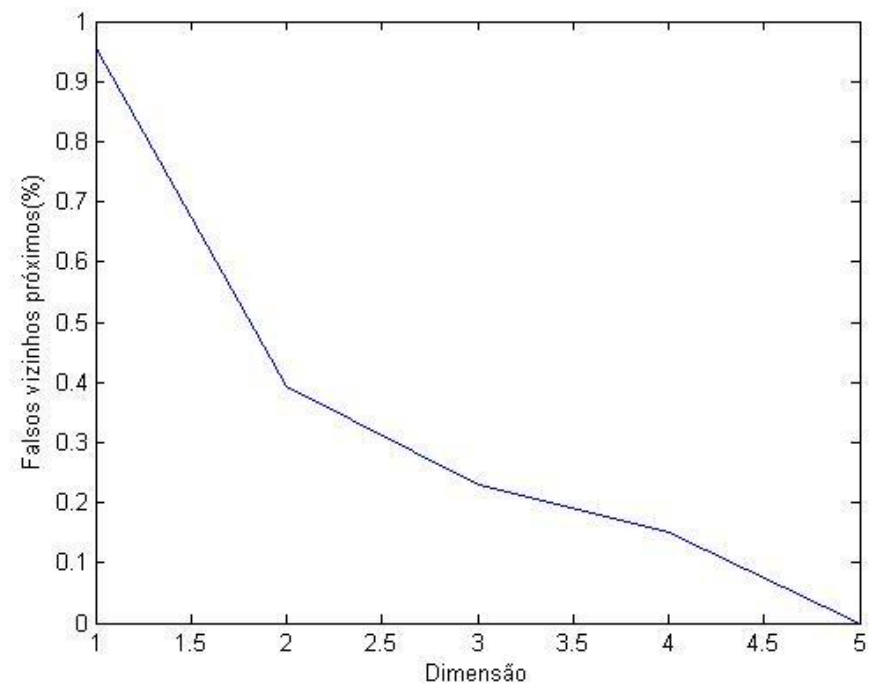

Figura 5. 37 - Porcentagem de falsos vizinhos próximos para a amostra de safira em $\mathrm{Z}=10.2 \mathrm{~nm}$ indica $m=5$ 


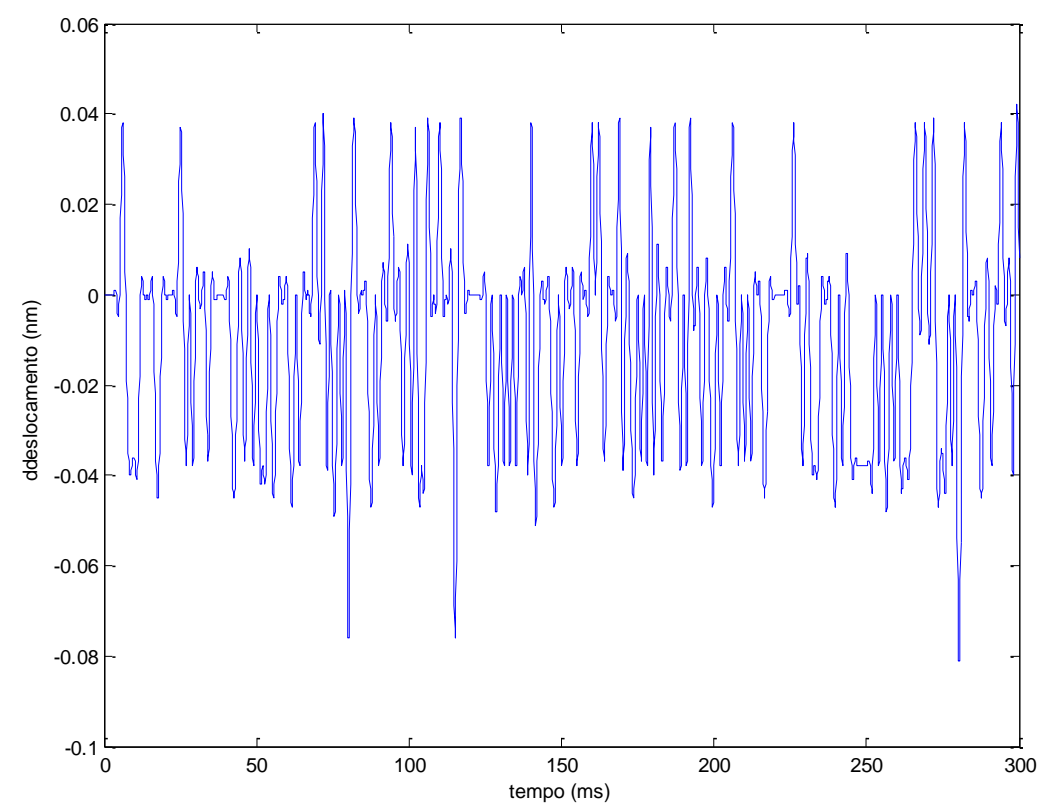

Figura 5. 38 - Deslocamento da microviga em $\mathrm{z}=10.2 \mathrm{~nm}$

A figura 5.39 mostra o retrato de fase através da reconstrução de espaço de estados pelo método das coordenadas defasadas e a figura 5.40 mostra o retrato de fase pelo método das derivadas que se diferem fundamentalmente pela inclinação que o retrato reconstruído ocasiona, e pelo fato do retrato de fase reconstruído apresentar com mais clareza os atratores caóticos e não ser altamente sensível ao ruído como o método das derivadas.

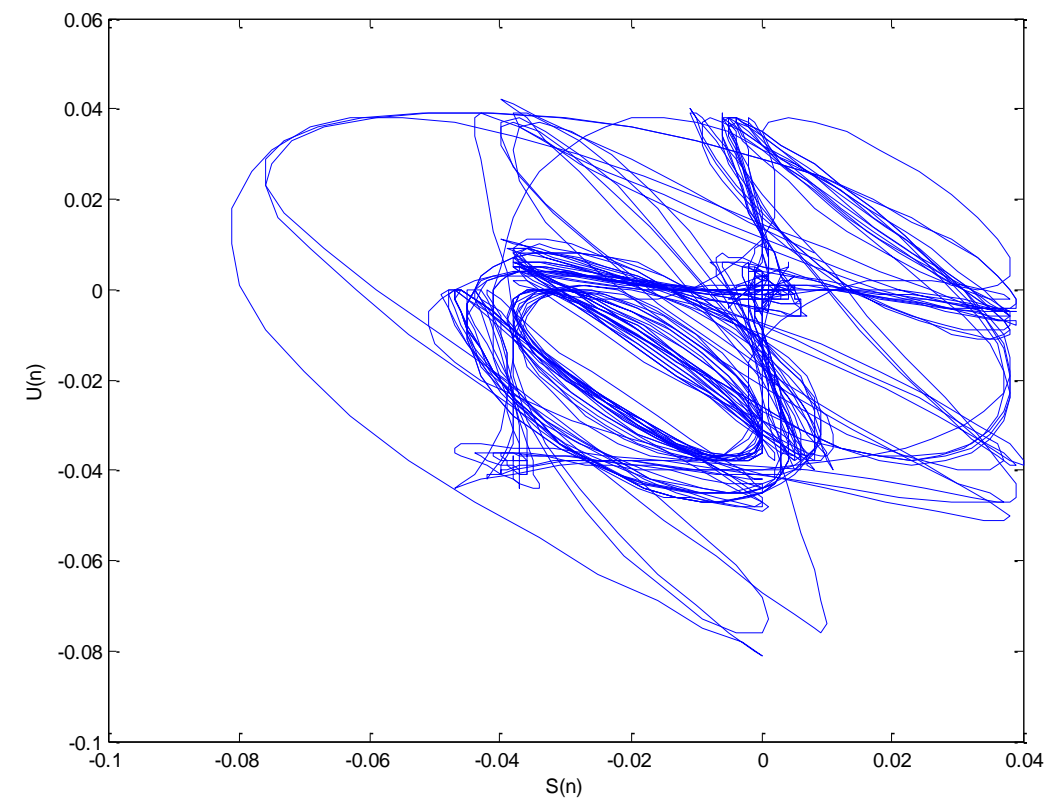

Figura 5. 39 - Retrato de fase da série temporal em safira em $\mathrm{Z}=10.2 \mathrm{~nm}$ reconstruída com $\mathrm{m}=5$ e $\tau=13$ 


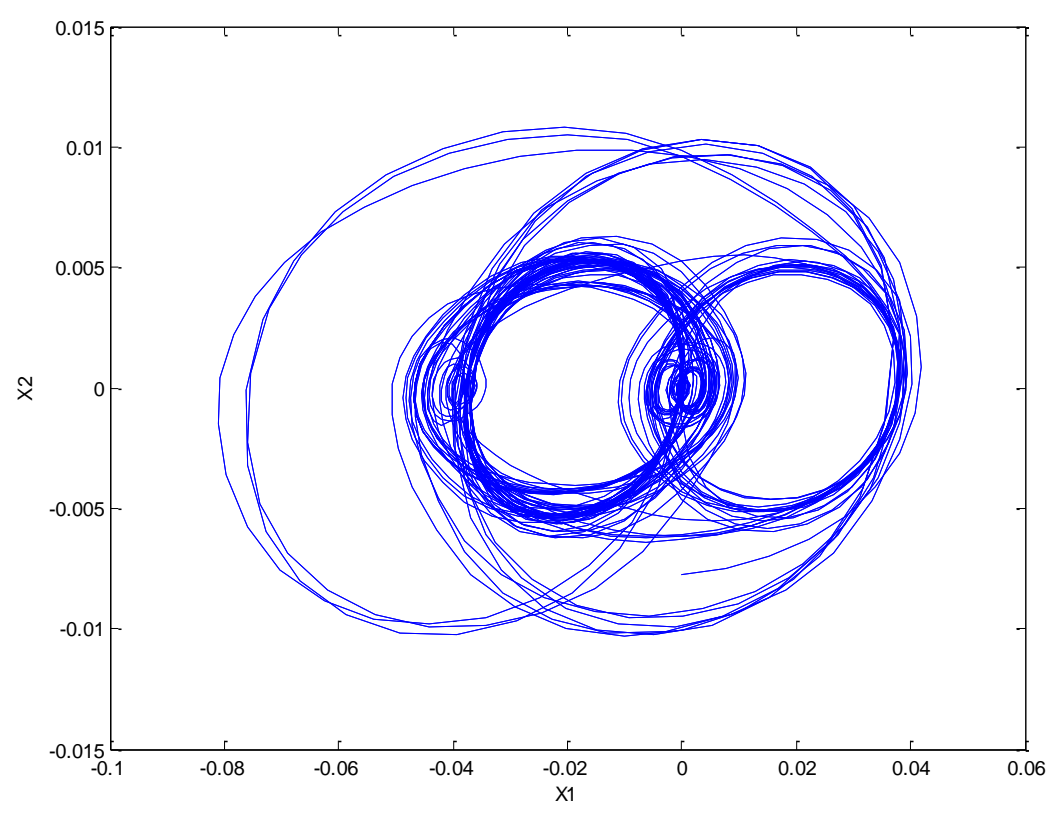

Figura 5. 40 - Reconstrução de espaço da série de safira em $\mathrm{Z}=10.2 \mathrm{~nm}$ pelo método das derivadas

A figura 5.41 mostra a FFT da série temporal obtida com altura $10.2 \mathrm{~nm}$ com a característica padrão para séries caóticas. A FFT tem picos altos em baixa frequência e depois estabiliza em uma amplitude menor a altas frequências (Guzzo, 2006).

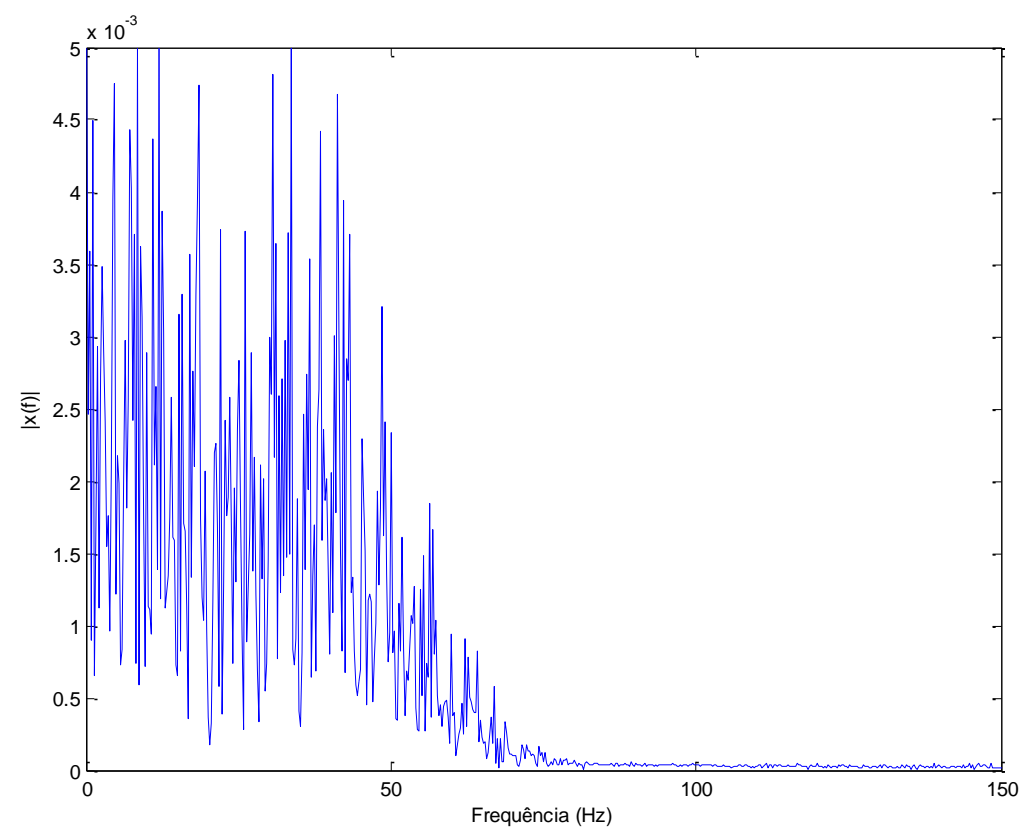

Figura 5. 41 - FFT da série temporal em safira na altura $Z=10.2 \mathrm{~nm}$ 
Para $\mathrm{Z}=316.2 \mathrm{~nm}$ a série temporal captada na amostra de safira se apresenta com menor valor de $K$ para o teste 0 -1. Calculando a informação mútua da série obtém-se a tabela 3 a seguir:

Tabela 4 - Informação mútua para série A em z=316.2nm

\begin{tabular}{|c|c|}
\hline$\tau$ & Informação mútua \\
\hline 0 & 2,151234 \\
\hline 1 & 1,368548 \\
\hline 2 & 1,057872 \\
\hline 3 & 0,8781043 \\
\hline 4 & 0,724635 \\
\hline 5 & 0,6602026 \\
\hline 6 & 0,6111874 \\
\hline 7 & 0,5627577 \\
\hline 8 & 0,4987392 \\
\hline 9 & 0,4691805 \\
\hline 10 & 0,4354394 \\
\hline 11 & 0,4364806 \\
\hline 12 & 0,3968927 \\
\hline 13 & 0,3848875 \\
\hline 14 & 0,3945276 \\
\hline 15 & 0,3966085 \\
\hline 16 & 0,3689351 \\
\hline 17 & 0,3370279 \\
\hline 18 & 0,2862198 \\
\hline 19 & 0,2936481 \\
\hline 20 & 0,3204889 \\
\hline
\end{tabular}




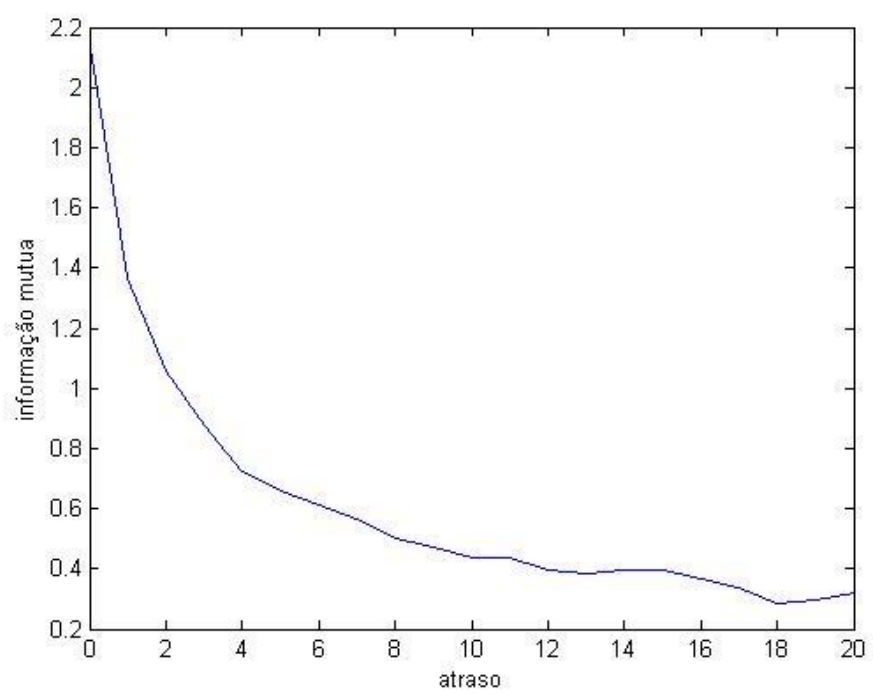

Figura 5. 42 - Informação mútua da série temporal da safira na altura $\mathrm{z}=316.2 \mathrm{~nm}$ com mínimo local em $\tau=10$

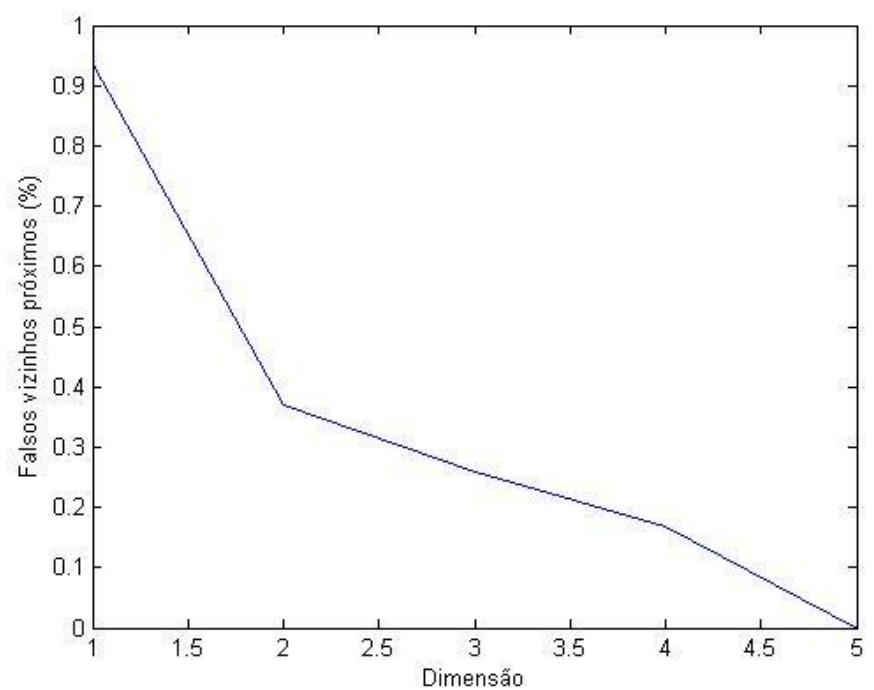

Figura 5. 43 - Dimensão da série em safira $Z=316.2 \mathrm{~nm}$ indica $m=5$ pelo método dos falsos vizinhos 


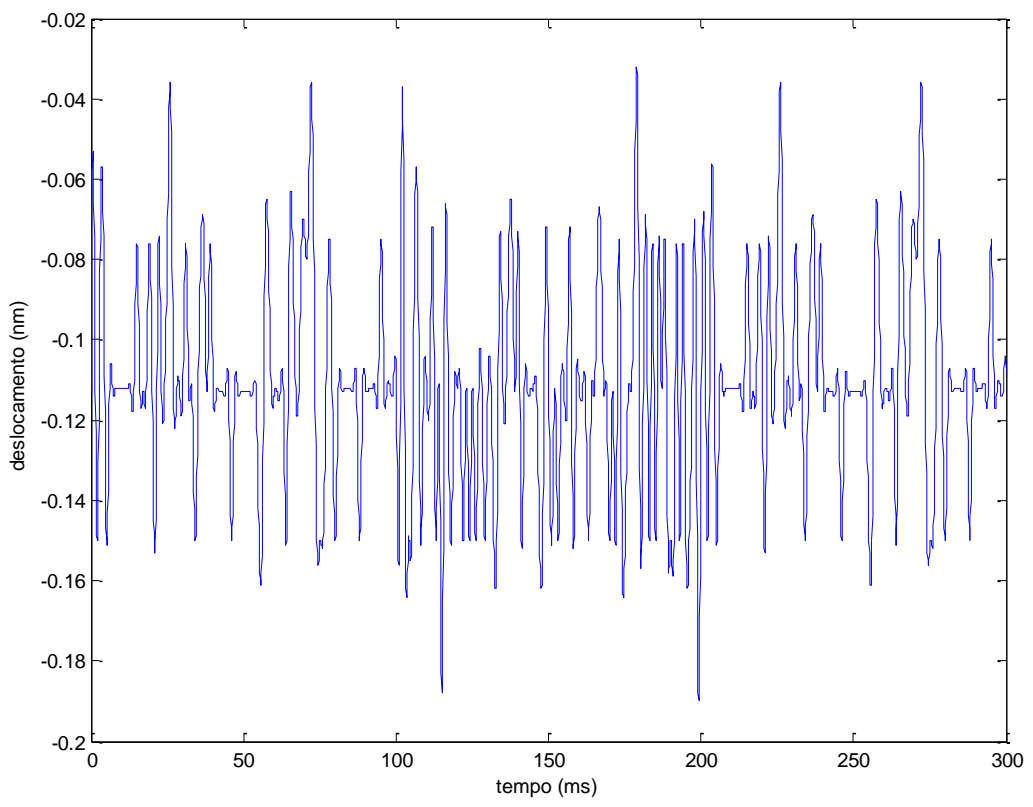

Figura 5. 44 - Deslocamento da microviga em $\mathrm{z}=316.2 \mathrm{nmm}$

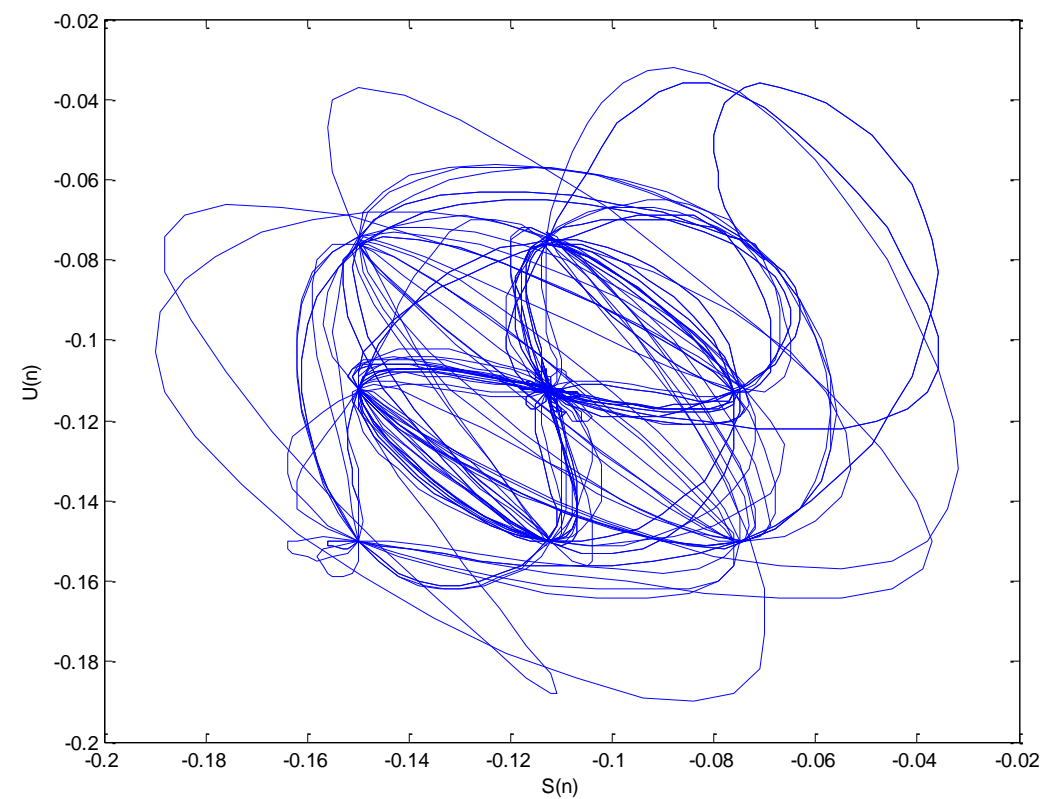

Figura 5. 45 - Retrato de fase da série temporal em safira em Z=316.2nm reconstruída com $\mathrm{m}=5$ e $\tau=10$ 


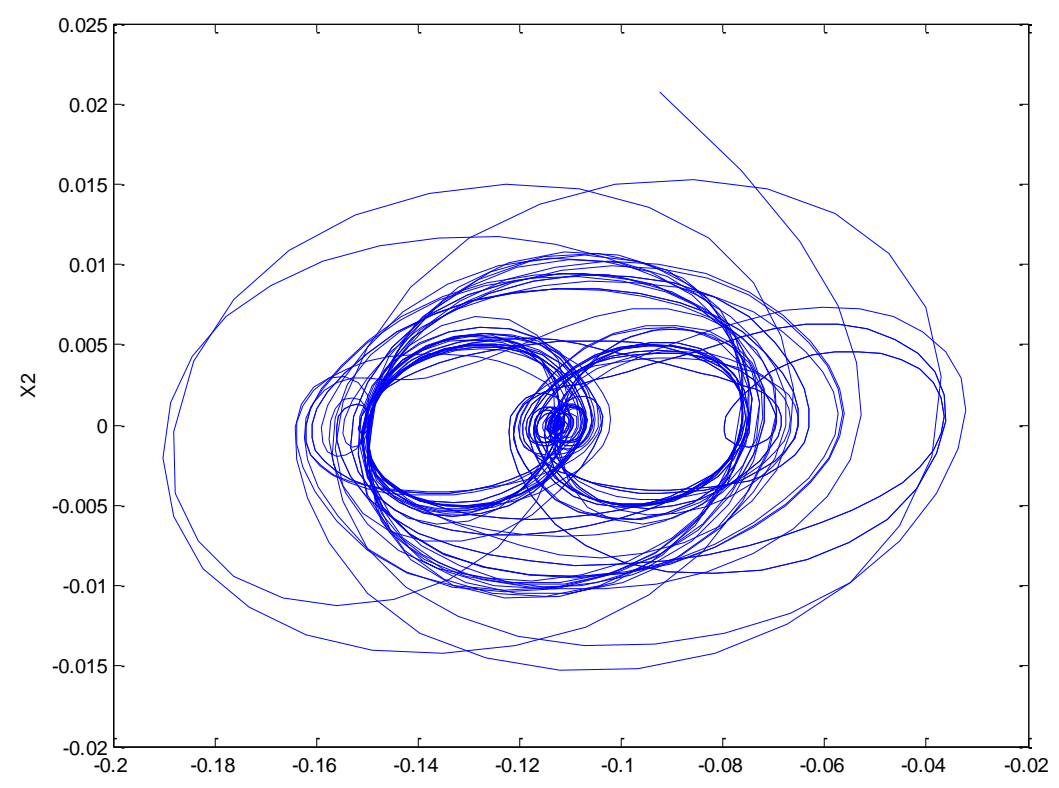

Figura 5. 46 - Reconstrução do espaço de estados para a série em safira em $Z=316.2 \mathrm{~nm}$ pelo método das derivadas

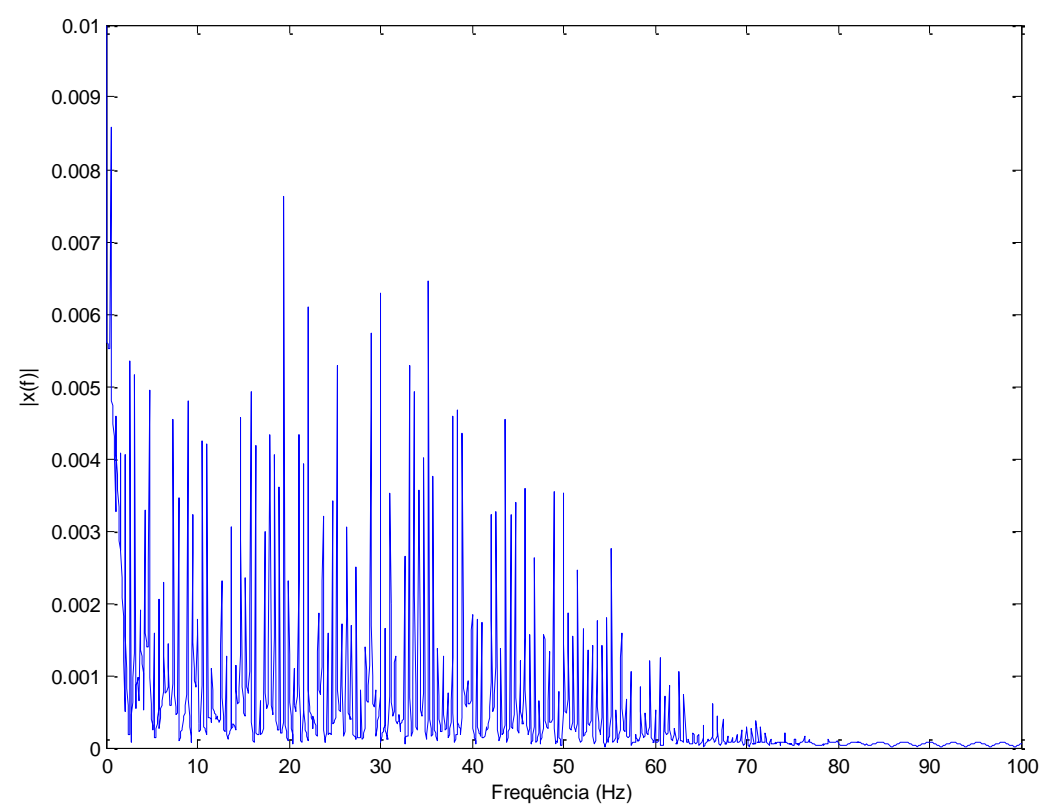

Figura 5. 47 - FFT da série temporal em safira em $Z=316.2 \mathrm{~nm}$ 


\subsubsection{SÉRIE TEMPORAL B}

A série temporal 'B' foi captada com a amostra de safira, 'scan size' no valor zero, constante elástica de $0,4 \mathrm{~N} / \mathrm{m}$ refente a ponteira triangular, sens. amp/sens $=60 \mathrm{~nm} / \mathrm{V}$, amplitude setpoint $=200 \mathrm{mV}$, drive setpoint $=307.6 \mathrm{mV}$, e temperatura $=22.4^{\circ} \mathrm{C}$.

Tabela 5 - Tabela com teste $0-1$ e escala indexada para série ' $b$ '

\begin{tabular}{c|c|c|c|c|c|c|c|c}
\hline $\mathbf{Z}$ & $\mathbf{K}$ & $\begin{array}{c}\text { LAA } \\
\text { INDE } \\
\text { XADA }\end{array}$ & $\mathbf{Z}$ & $\mathbf{K}$ & $\begin{array}{c}\text { LA } \\
\text { INDE } \\
\text { XADA }\end{array}$ & $\mathbf{Z}$ & $\mathbf{K}$ & $\begin{array}{c}\text { ESCA } \\
\text { LA } \\
\text { INDE } \\
\mathbf{X A D A}\end{array}$ \\
\hline 2 & 0,99554 & 0,99945 & 249 & 0,97055 & 0,99946 & 496 & 0,96269 & 0,99946 \\
21 & 0,9954 & 0,99944 & 268 & 0,96283 & 0,99946 & 515 & 0,9639 & 0,99946 \\
40 & 0,99184 & 0,99943 & 287 & 0,97002 & 0,99946 & 534 & 0,9242 & 0,99946 \\
59 & 0,99568 & 0,99946 & 306 & 0,96237 & 0,99946 & 553 & 0,5854 & 0,99945 \\
78 & 0,96996 & 0,99943 & 325 & 0,96117 & 0,99944 & 572 & 0,88327 & 0,99945 \\
97 & 0,95798 & 0,99943 & 344 & 0,96784 & 0,99946 & 591 & 0,85133 & 0,99945 \\
116 & 0,95325 & 0,99943 & 363 & 0,97949 & 0,99945 & 610 & 0,81161 & 0,99945 \\
135 & 0,94604 & 0,99943 & 382 & 0,96484 & 0,99944 & 629 & 0,90437 & 0,99946 \\
154 & 0,91504 & 0,99943 & 401 & 0,97875 & 0,99945 & 648 & 0,90775 & 0,99944 \\
173 & 0,94972 & 0,99943 & 420 & 0,93764 & 0,99946 & & & \\
192 & 0,92445 & 0,99943 & 439 & 0,97064 & 0,99946 & & & \\
211 & 0,97752 & 0,99943 & 458 & 0,9685 & 0,99946 & & & \\
230 & 0,97422 & 0,99946 & 477 & 0,96281 & 0,99946 & & & \\
\hline
\end{tabular}




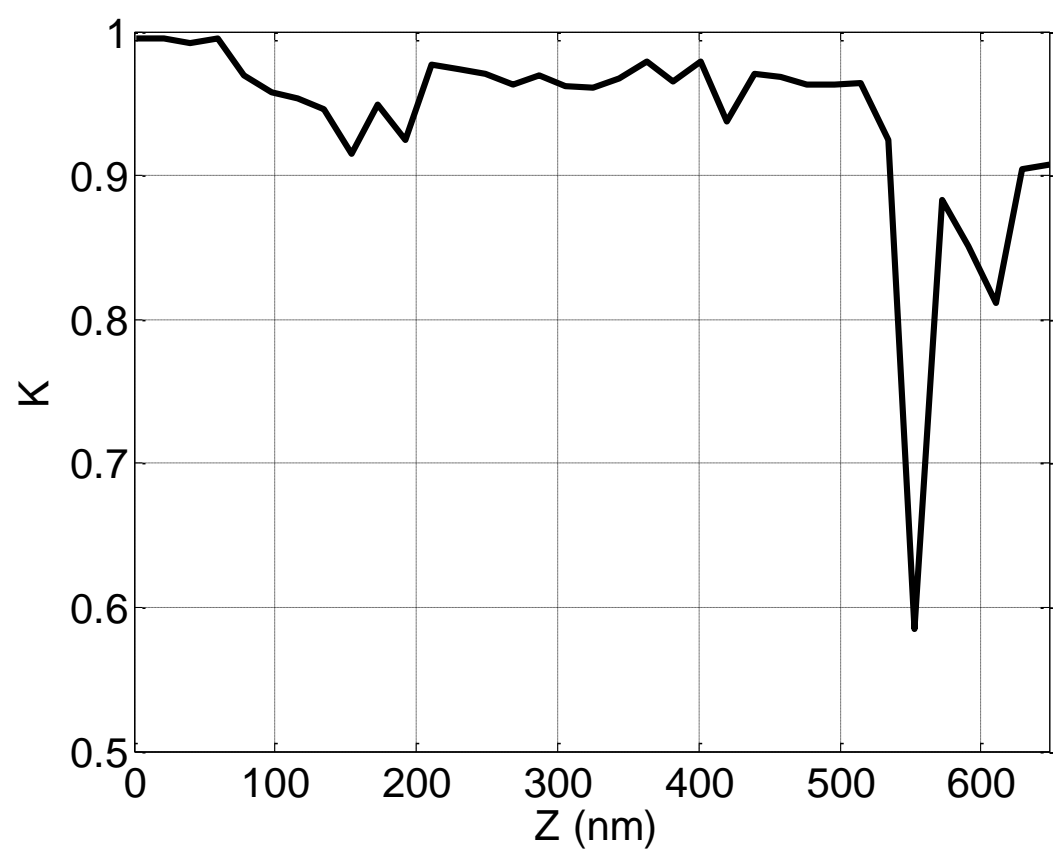

Figura 5. 48 - Gráfico de variação de $K$ para série 'b'.

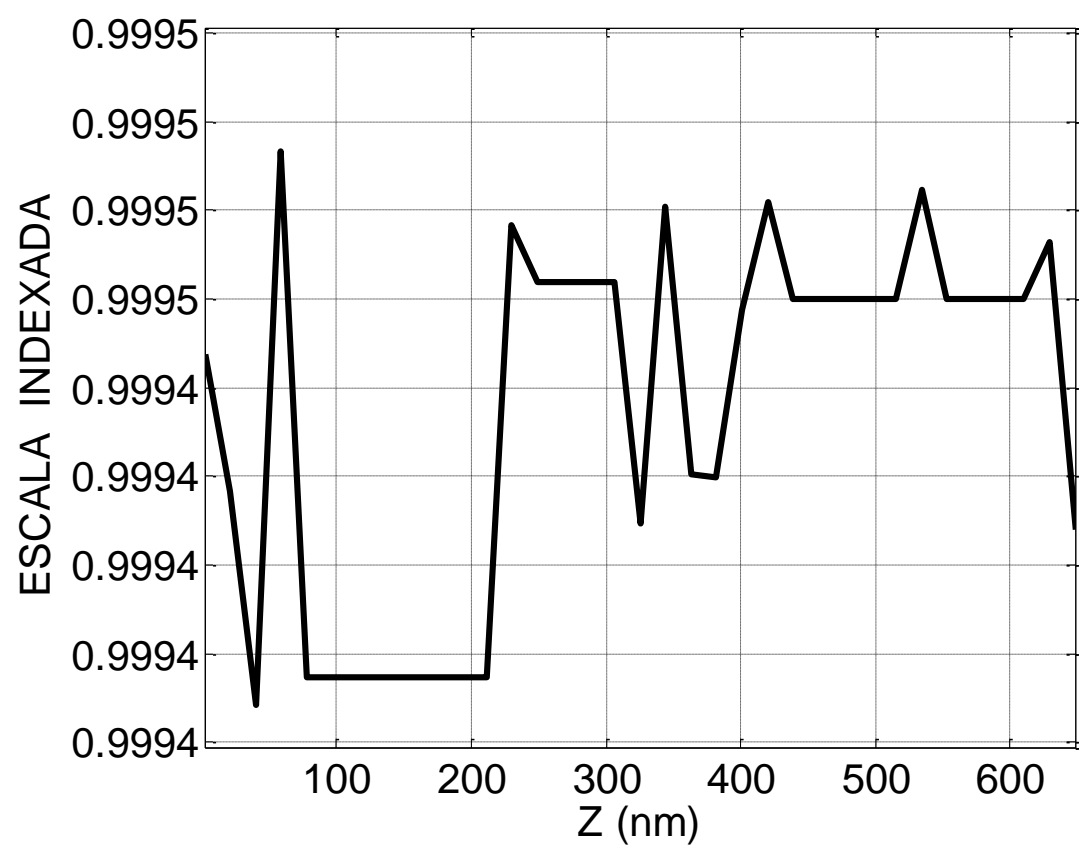

Figura 5. 49 - Escala indexada para a série ' $b$ '

A figura 5.8 mostra uma região em tem que o valor $k$ do teste 0 -1 é bem pequeno. Para $\mathrm{Z}=59 \mathrm{~nm}$ a série temporal analisada em safira com ponta triangular atinge, no teste $0-1$, o valor k máximo. Já a escala indexada mostra todos as séries com um valor muito próximo a 1, o que indica altíssimo grau de caos nas séries produzida pelo experimento B. 
Tabela 6 - Informação mútua para série $\mathrm{B}$ em z=59.2nm

\begin{tabular}{|c|c|}
\hline$\tau$ & Informação mútua \\
\hline 0 & 2.259097 \\
\hline 1 & 1.497772 \\
\hline 2 & 1.156008 \\
\hline 3 & 0.8925719 \\
\hline 4 & 0.684843 \\
\hline 5 & 0.5605905 \\
\hline 6 & 0.4175419 \\
\hline 7 & 0.2974836 \\
\hline 8 & 0.2307642 \\
\hline 9 & 0.1911435 \\
\hline 10 & 0.1795733 \\
\hline 11 & 0.1561899 \\
\hline 12 & 0.1042034 \\
\hline 13 & 0.09602411 \\
\hline 14 & 0.09320763 \\
\hline 15 & 0.09078617 \\
\hline 16 & 0.09146622 \\
\hline 17 & 0,09265216 \\
\hline 18 & 0,0933502 \\
\hline 19 & 0,1121988 \\
\hline 20 & 0,1230974 \\
\hline
\end{tabular}

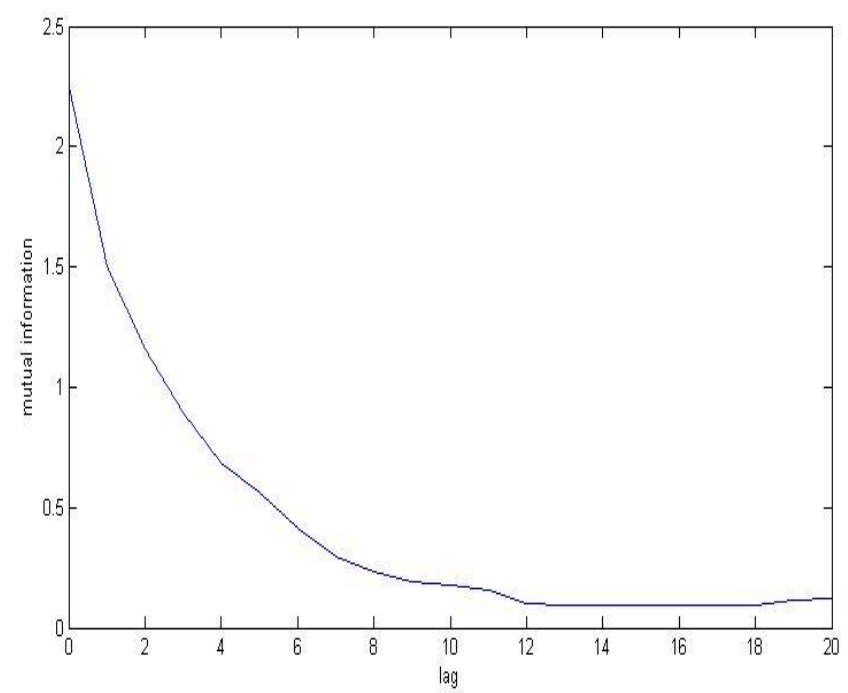

Figura 5. 50 - Informação mútua da série temporal da safira com ponta triangular na altura $\mathrm{z}=59.2 \mathrm{~nm}$ com mínimo local em $\tau=15$ 


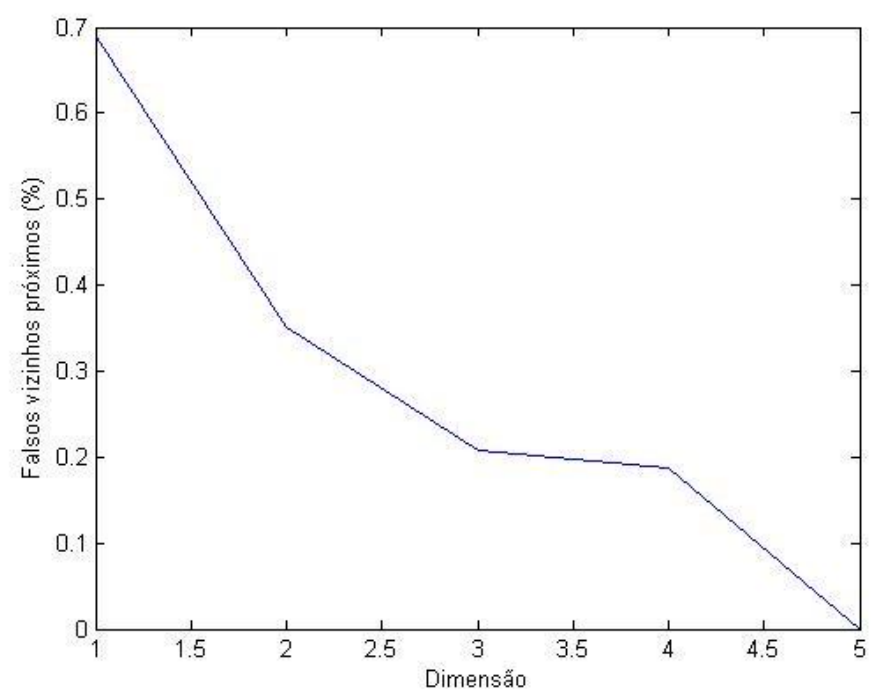

Figura 5. 51 - Dimensão de imersão calculada para a série de safira com ponta triangular em $\mathrm{Z}=59.2 \mathrm{~nm}$ pelo método dos falsos vizinhos próximos

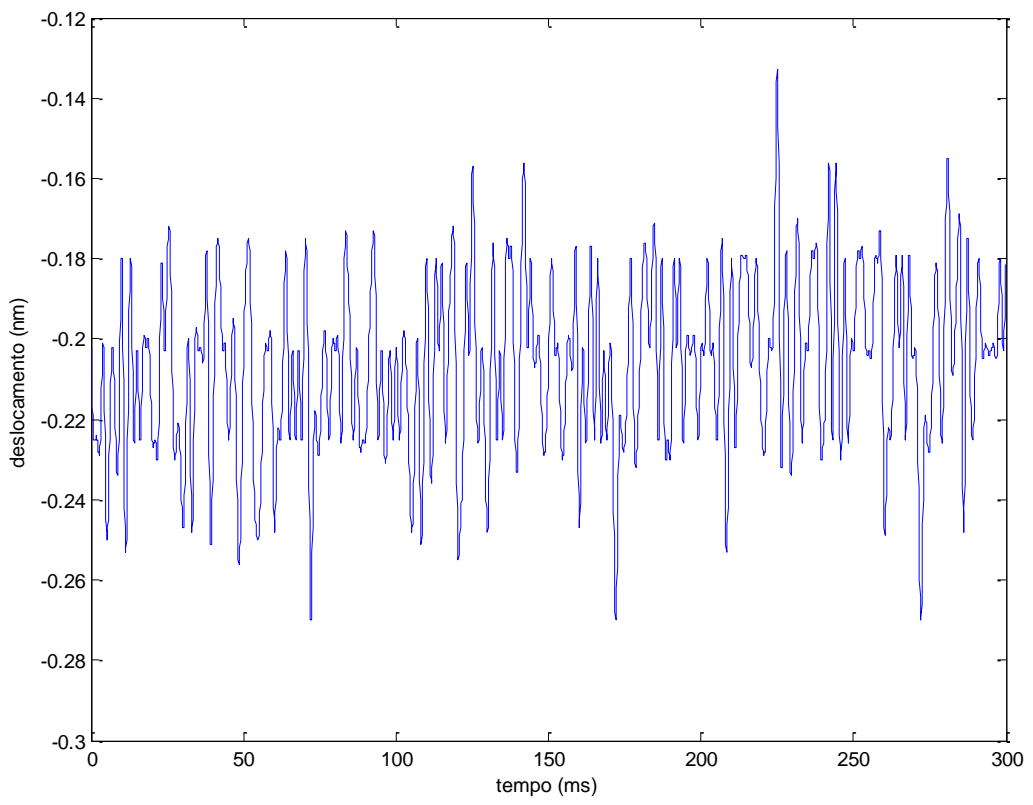

Figura 5. 52 - Deslocamento da microviga em $z=59.2 \mathrm{~nm}$ 


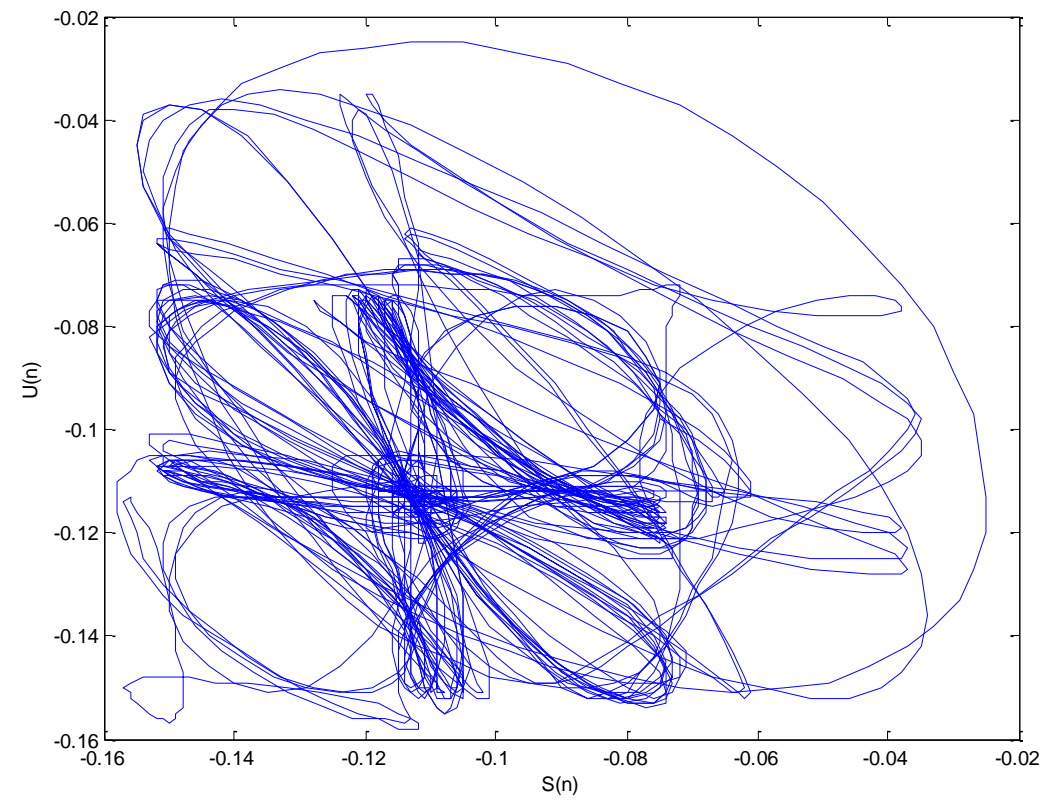

Figura 5. 53 - Retrato de fase da série temporal em safira com ponta triangular em $\mathrm{Z}=59.2 \mathrm{~nm}$ reconstruída com $\mathrm{m}=5$ e $\tau=13$

A figura 5.53 mostra o retrato de fase reconstruído com diversos atratores caóticos, enquanto que na figura 5.54 o retrato de fase pode ser 'rotulada' de período 3 , mesmo observando a FFT em Fig 5.55 mostrando o alto grau caótico. Daí a importância de se utilizar o método das coordenadas defasadas.

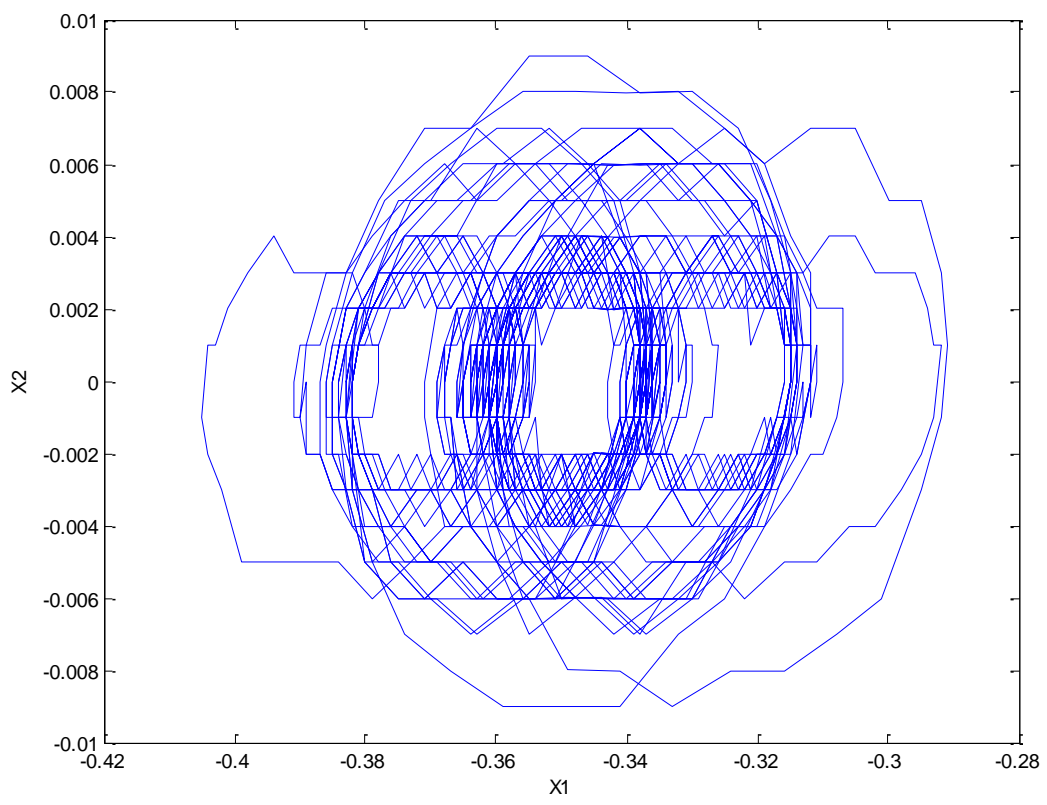

Figura 5. 54 - Reconstrução do espaço de estados da série em safira com ponta triangular em $\mathrm{Z}=59.2 \mathrm{~nm}$ pelo método das derivadas 


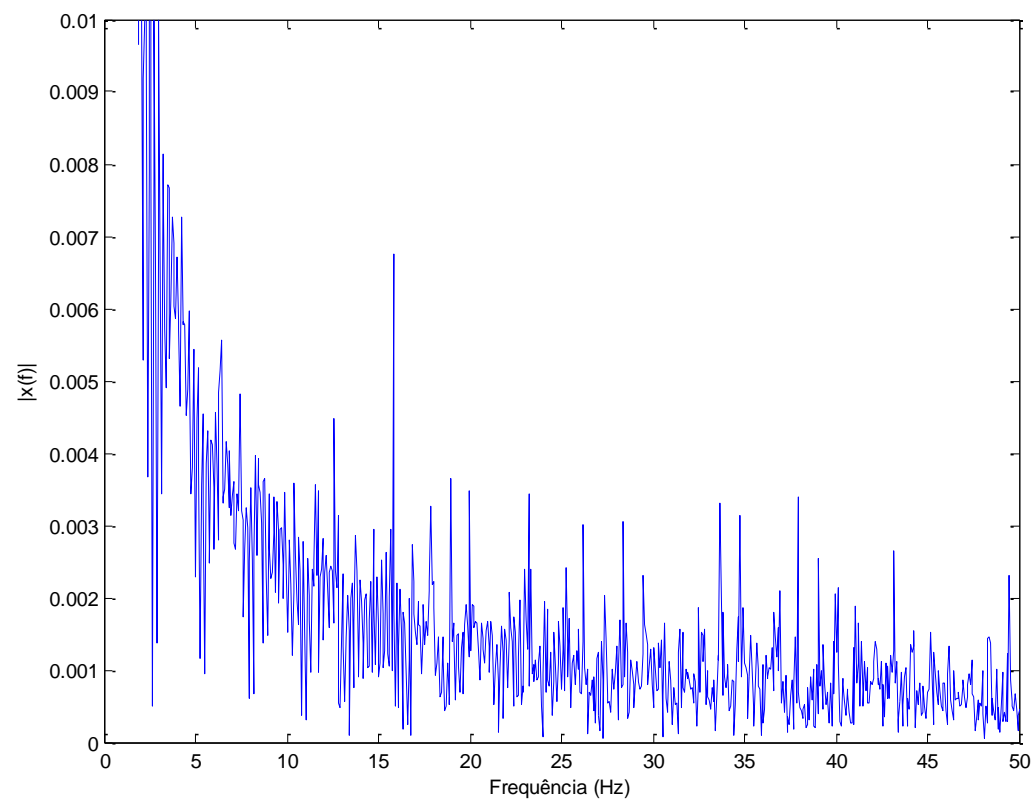

Figura 5. 55 - FFT da série de safira com ponta triangular em $Z=59.2 \mathrm{~nm}$

Para a altura $Z=553 \mathrm{~nm}$ o teste $0-1$ na amostra de safira com ponta triangular indica o valor $K$ mínimo no experimento $\mathrm{B}$

Tabela 7 - Informação mútua para a série B em z=553nm

\begin{tabular}{|c|c|}
\hline$\tau$ & Informação mútua \\
\hline 0 & 2.399268 \\
\hline 1 & 1.546911 \\
\hline 2 & 1.196043 \\
\hline 3 & 1.018717 \\
\hline 4 & 0.851286 \\
\hline 5 & 0.7358733 \\
\hline 6 & 0.6560929 \\
\hline 7 & 0.561962 \\
\hline 8 & 0.5038457 \\
\hline 9 & 0.4928768 \\
\hline 10 & 0.4532175 \\
\hline 11 & 0.4346896 \\
\hline 12 & 0.3434659 \\
\hline 13 & 0.3501546 \\
\hline 14 & 0.3905373 \\
\hline 15 & 0.3926602 \\
\hline
\end{tabular}




\begin{tabular}{|l|l|}
\hline 16 & 0.3279563 \\
\hline 17 & 0.2952878 \\
\hline 18 & 0.2621958 \\
\hline 19 & 0.2750837 \\
\hline 20 & 0.2758720 \\
\hline
\end{tabular}

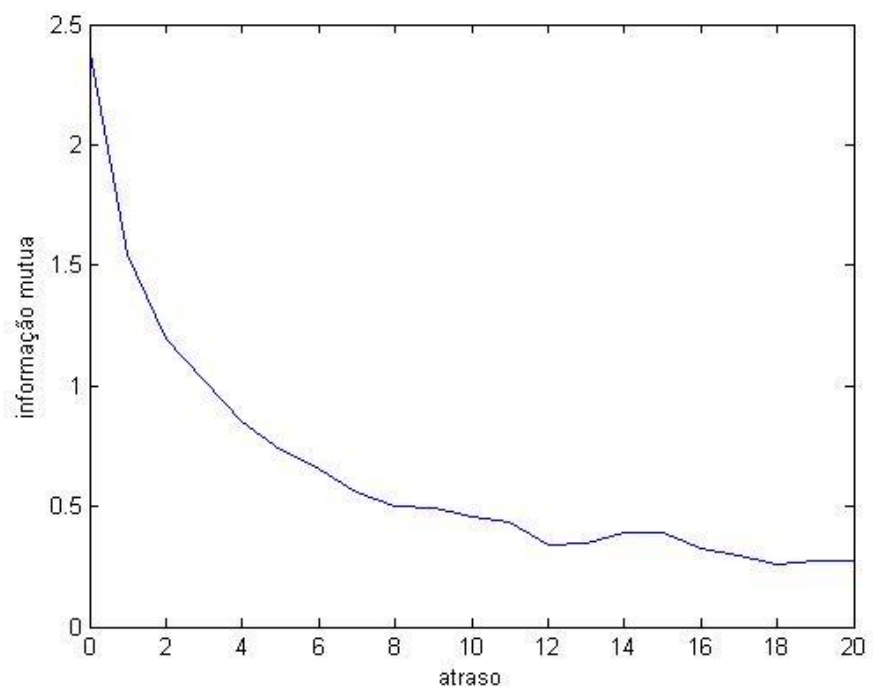

Figura 5. 56 - Informação mútua da série temporal da safira na altura z $=553 \mathrm{~nm}$ com mínimo local em $\tau=10$

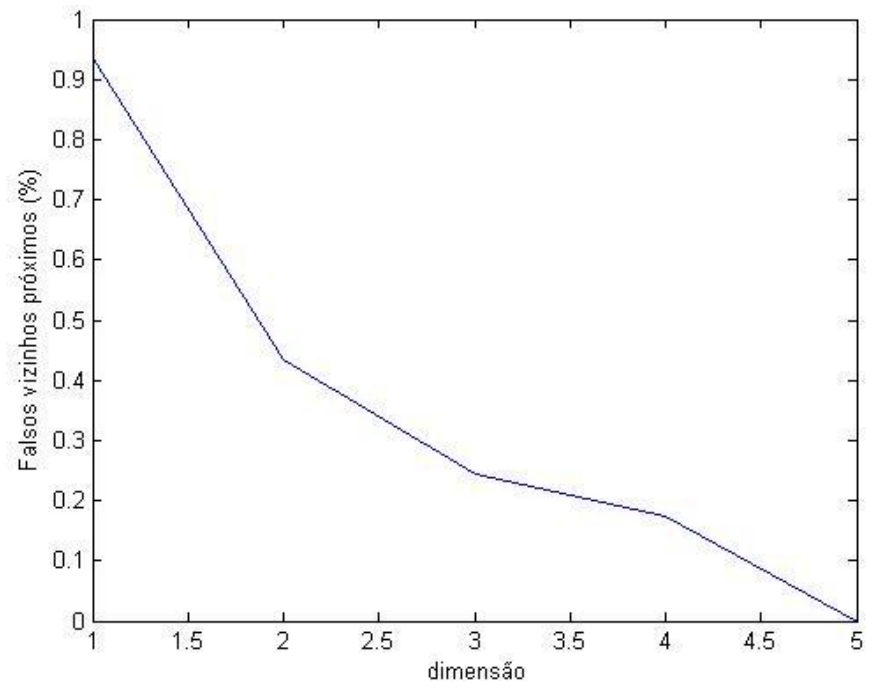

Figura 5. 57 - Dimensão de imersão calculado para a série analisada em safira com ponta triangular em $Z=553 \mathrm{~nm}$ pelo método dos falsos vizinhos 


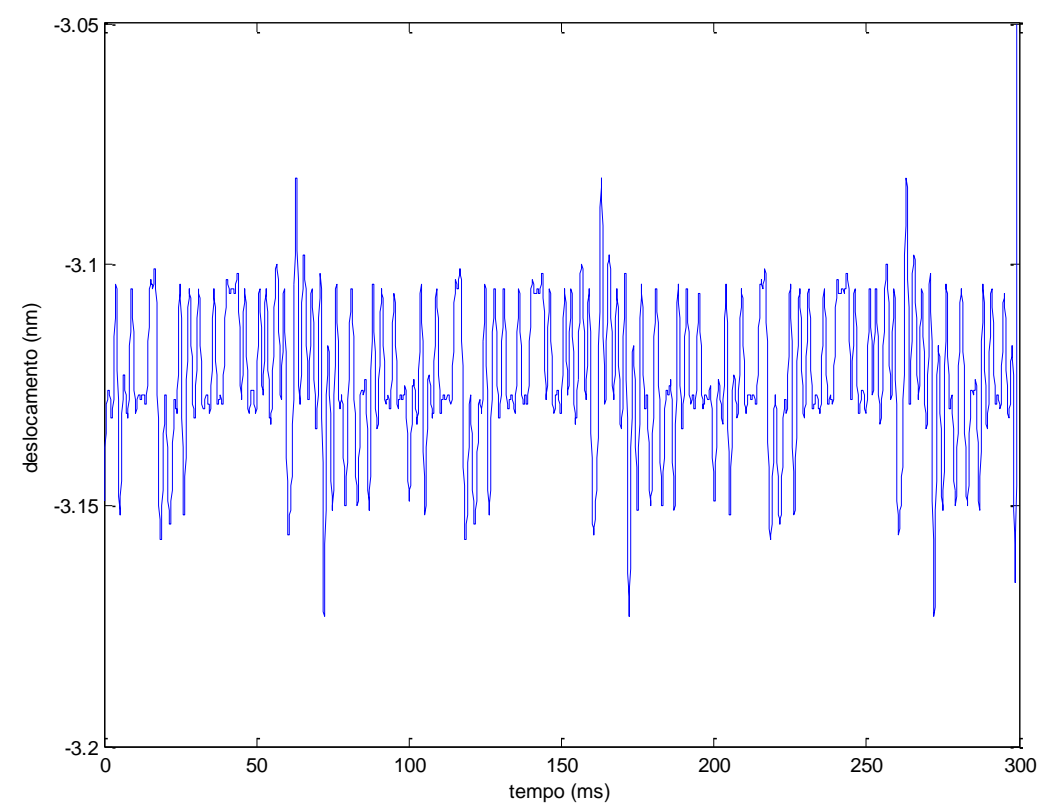

Figura 5. 58 - Deslocamento da microviga em $\mathrm{z}=553 \mathrm{~nm}$

Ao se comparar a figura 5.59 com a figura 5.60 observa-se uma certa divergência entre o teor caótico da série. A fig. 5.59 apresenta muitos atratores caóticos enquanto a fig. 5.60 tem período 2. Observando a FFT em Fig 5.61 de cunho caótico reafirma o fato do retrato do retrato de fase reconstruído pelo método das coordenadas defasadas ser o melhor procedimento.

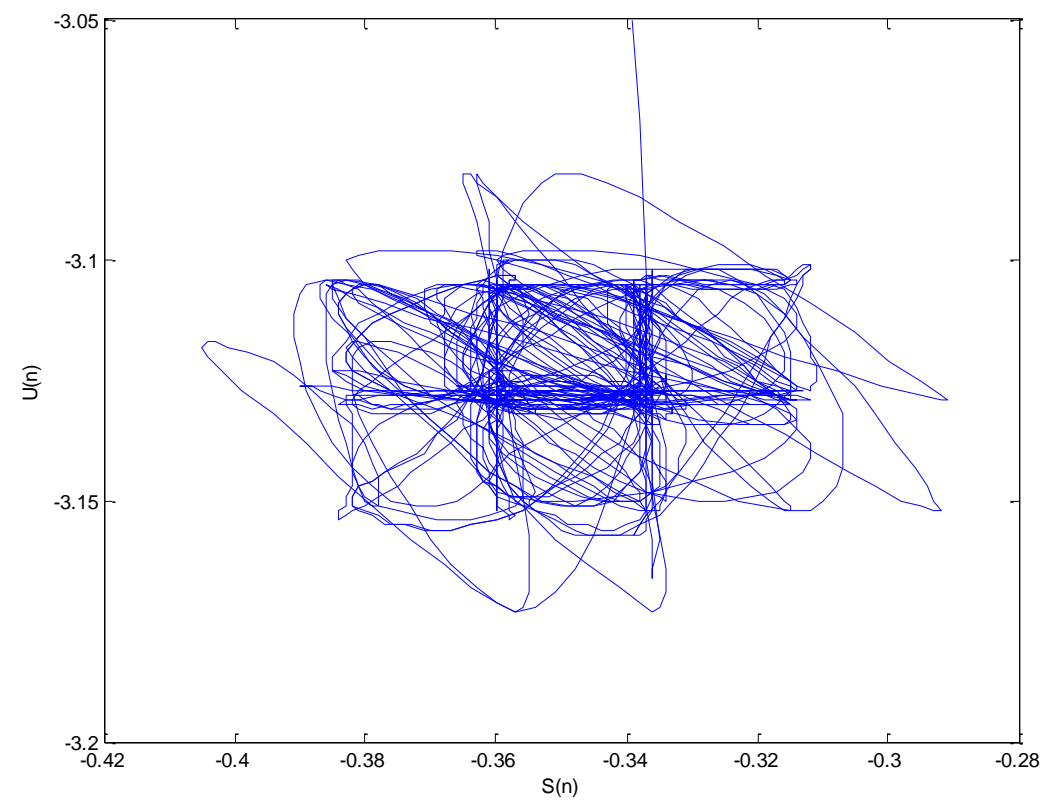

Figura 5. 59 - Retrato de fase da série temporal em safira com ponta triangular em $Z=553 \mathrm{~nm}$ reconstruída com $m=5$ e $\tau=13$ 


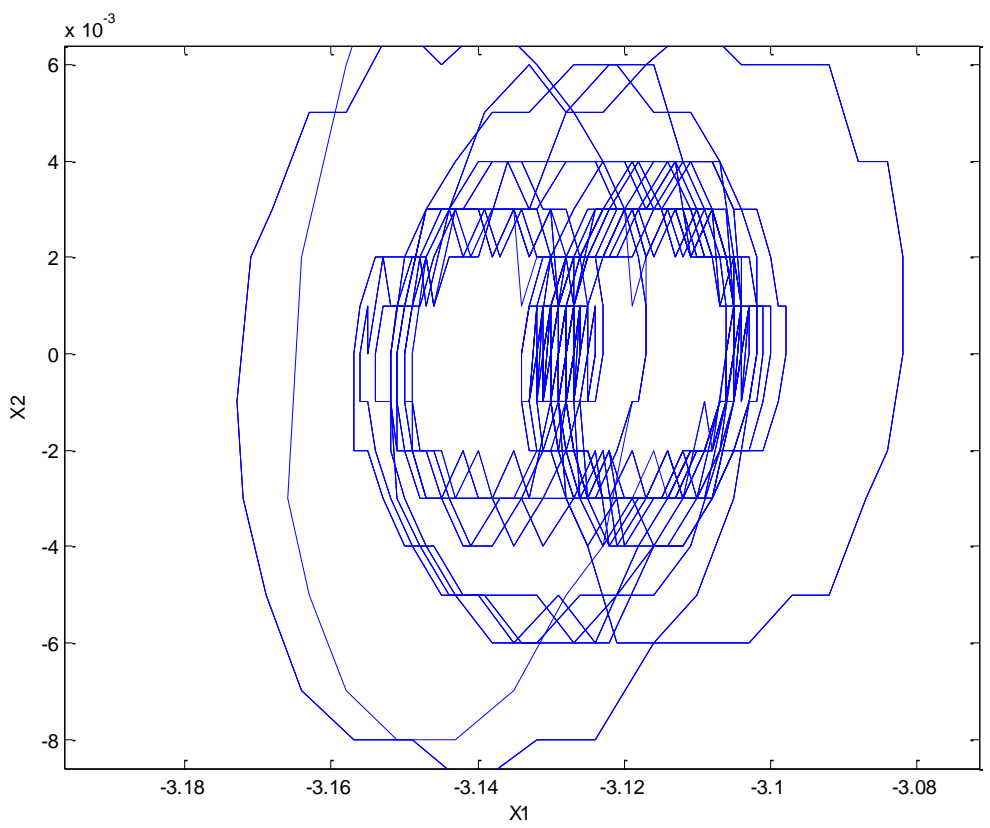

Figura 5. 60 - Retrato de fase da safira com ponta triangular em Z=553nm reconstruído pelo método das derivadas

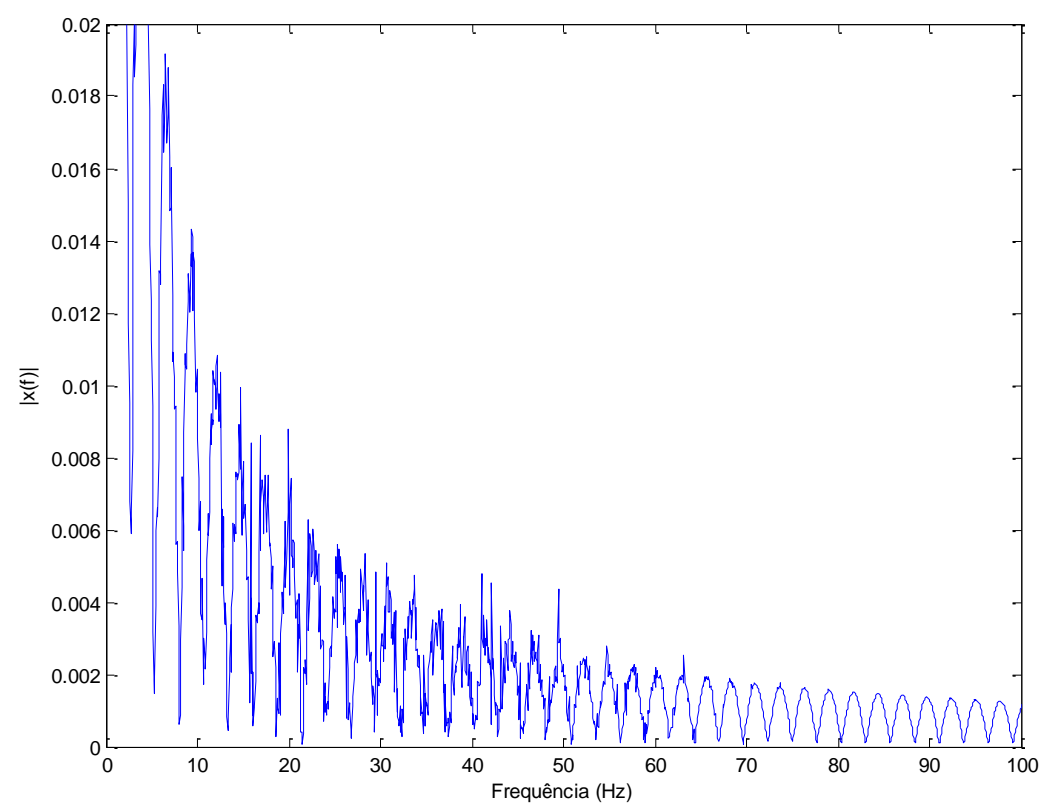

Figura 5. 61 - FFT da série produzida em safira com ponta triangular e Z=553nm 


\subsubsection{SÉRIE TEMPORAL C}

$\mathrm{O}$ experimento $\mathrm{C}$ foi produzido com corpo de teste de borracha, Scan size zero, constante elástica de $42 \mathrm{~N} / \mathrm{m}$, ponteira triangular, sens. amp/sens de $100 \mathrm{~nm} / \mathrm{V}$, amplitude setpoint de $149.99 \mathrm{mV}$, drive amplitude de $165 \mathrm{mV}$,e temperatura de $22.4^{\circ} \mathrm{C}$. A troca por borracha foi visando ter um contraste com um material duro escaneado (safira nos experimentos A, B e D). As forças de interação ponta amostra (força de Van der Waals e forças de capilaridade) são totalmente diferentes mas não é possível mensurá-las.

Tabela 8 - Tabela com teste $0-1$ e escala indexada para série ' $c$ '

\begin{tabular}{c|c|c|c|c|c|c|c|c}
\hline $\mathbf{Z}$ & $\mathbf{K}$ & $\begin{array}{c}\text { LACA } \\
\text { INDE } \\
\text { XADA }\end{array}$ & $\mathbf{Z}$ & $\mathbf{K}$ & $\begin{array}{c}\text { LA } \\
\text { INDE } \\
\text { XADA }\end{array}$ & $\mathbf{Z}$ & $\mathbf{K}$ & $\begin{array}{c}\text { ESCA } \\
\text { LA } \\
\text { INDE } \\
\mathbf{X A D A}\end{array}$ \\
\hline 2 & 0,99666 & 0,4582 & 249 & 0,98161 & 0,99959 & 496 & 0,98424 & 0,99942 \\
21 & 0,99677 & 0,99994 & 268 & 0,98651 & 0,99959 & 515 & 0,98745 & 0,99942 \\
40 & 0,99672 & 0,89931 & 287 & 0,98162 & 0,99959 & 534 & 0,9885 & 0,99947 \\
59 & 0,99653 & 0,99903 & 306 & 0,9815 & 0,99959 & 553 & 0,98823 & 0,99942 \\
78 & 0,99211 & 0,99955 & 325 & 0,9933 & 0,99947 & 572 & 0,98789 & 0,99942 \\
97 & 0,99371 & 0,99955 & 344 & 0,99423 & 0,99957 & 591 & 0,98202 & 0,99942 \\
116 & 0,99239 & 0,99955 & 363 & 0,99661 & 0,99951 & 610 & 0,98434 & 0,99942 \\
135 & 0,99407 & 0,99955 & 382 & 0,99789 & 0,99945 & 629 & 0,99139 & 0,99947 \\
154 & 0,99135 & 0,99955 & 401 & 0,99115 & 0,99949 & 648 & 0,9871 & 0,9994 \\
173 & 0,99203 & 0,99955 & 420 & 0,99265 & 0,99946 & & & \\
192 & 0,99333 & 0,99955 & 439 & 0,98603 & 0,99942 & & & \\
211 & 0,99779 & 0,99955 & 458 & 0,98814 & 0,99942 & & & \\
230 & 0,98766 & 0,99959 & 477 & 0,98806 & 0,99942 & & & \\
\hline
\end{tabular}

A figura 5.62- mostra regiões extremamente caóticas para a série $\mathrm{C}$, com todos os valores acima de 0.98 . Se comparado com a série $\mathrm{A}$, as séries produzidas no experimento $\mathrm{C}$ são em geral menos caóticas, já que em 'A' o teste estabelece valores próximos da faixa de 0.99. O resultado credita o fato das mudanças, de amostra, da safira para borracha. 


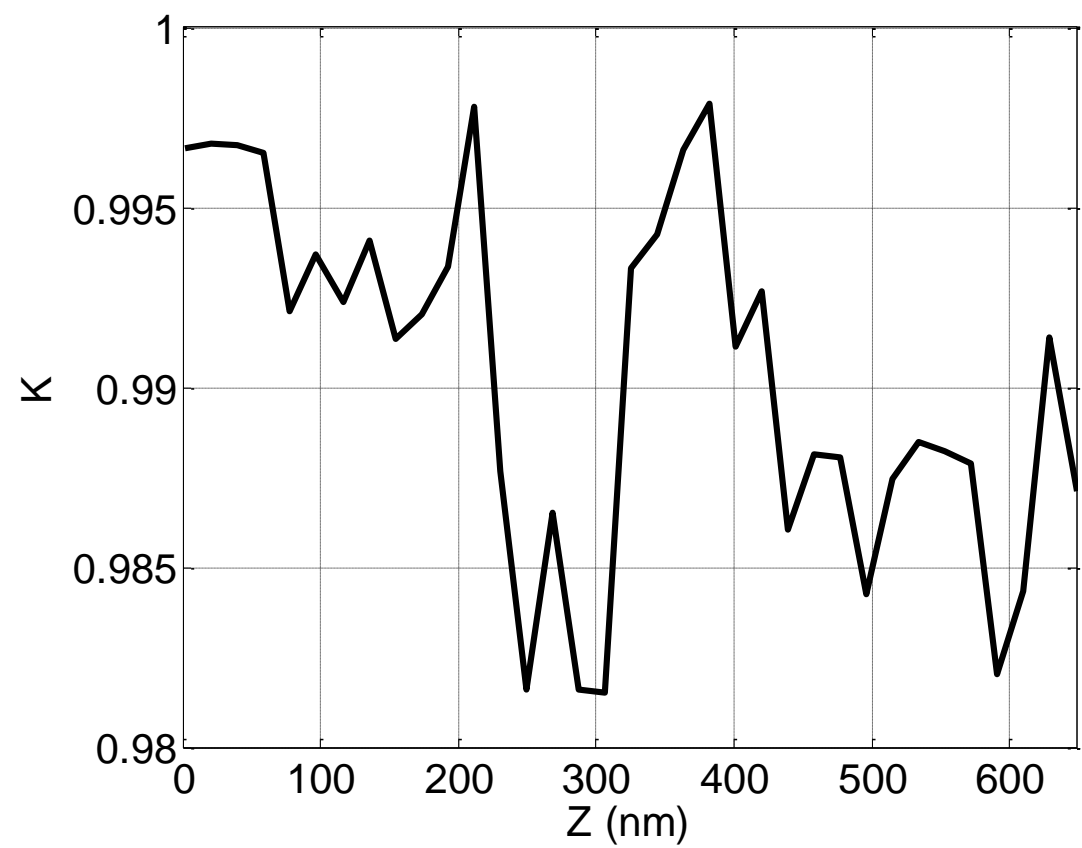

Figura 5. 62 - Gráfico de variação de $K$ para série 'c'

O teste da escala indexada mostra um resultado diferente do resultado do teste $0-1$, pois em z próximo de zero, o experimento apresenta valores que indicam movimentos caóticos mas aumentando os valores de $\mathrm{z}$, o teste da escala apresenta ainda mais movimentos caóticos. Em especial, neste experimento C, a série com maior valor $\mathrm{K}$ do teste 0 -1 não está próxima à amostra (altura $\mathrm{z}$ pequena) e se encontra próxima a mediana das alturas realizadas.

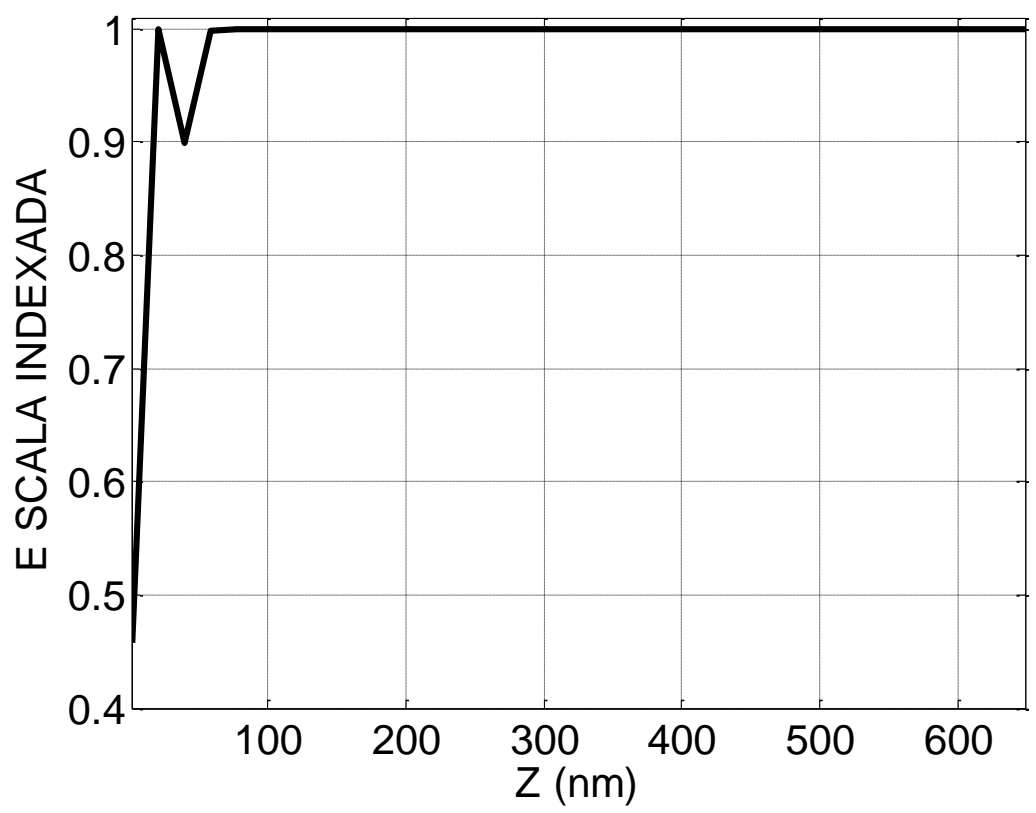

Figura 5. 63 - Teste da escala indexada para a série 'c' 
Para $\mathrm{z}=306 \mathrm{~nm}$ a série temporal analisada na borracha e ponta triangular tem o valor $\mathrm{k}$ do teste 0 -1 o seu valor mínimo.

Tabela 9 - Informação mútua para a série $\mathrm{C}$ em z=306nm

\begin{tabular}{|c|c|}
\hline$\tau$ & Informação mútua \\
\hline 0 & 2.546698 \\
\hline 1 & 1.654751 \\
\hline 2 & 1.253758 \\
\hline 3 & 0.9897439 \\
\hline 4 & 0.7815548 \\
\hline 5 & 0.6420641 \\
\hline 6 & 0.5354658 \\
\hline 7 & 0.4366172 \\
\hline 8 & 0.3890649 \\
\hline 9 & 0.4150562 \\
\hline 10 & 0.3881257 \\
\hline 11 & 0.3445067 \\
\hline 12 & 0.3290429 \\
\hline 13 & 0.3575629 \\
\hline 14 & 0.3773131 \\
\hline 15 & 0.3544361 \\
\hline 16 & 0.3198972 \\
\hline 17 & 0.330764 \\
\hline 18 & 0,3099948 \\
\hline 19 & 0,3027435 \\
\hline 20 & 0,275872 \\
\hline
\end{tabular}




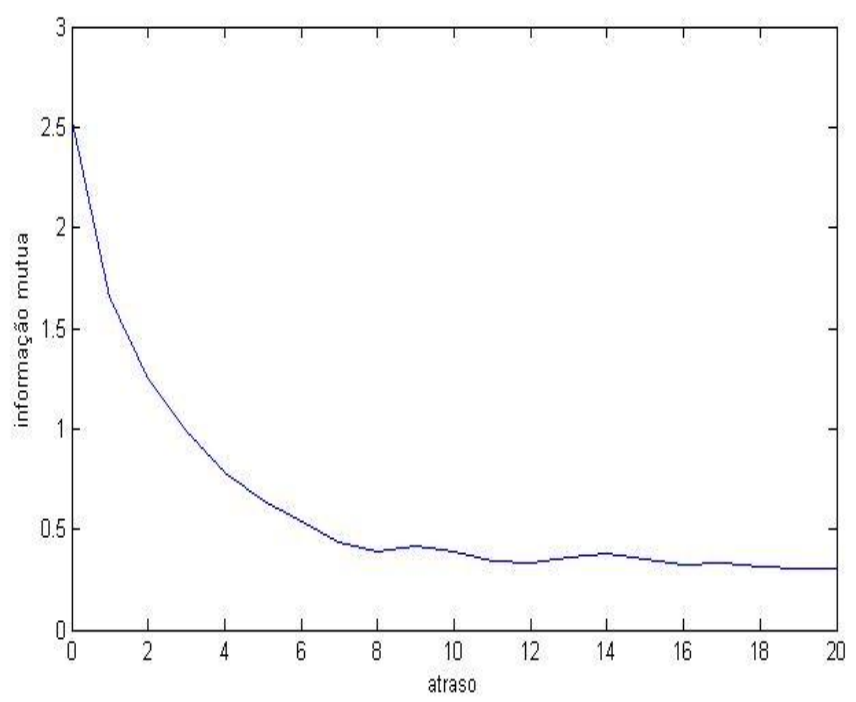

Figura 5. 64- Informação mútua da série temporal analisada em borracha na altura Z=306nm com mínimo local em $\tau=8$

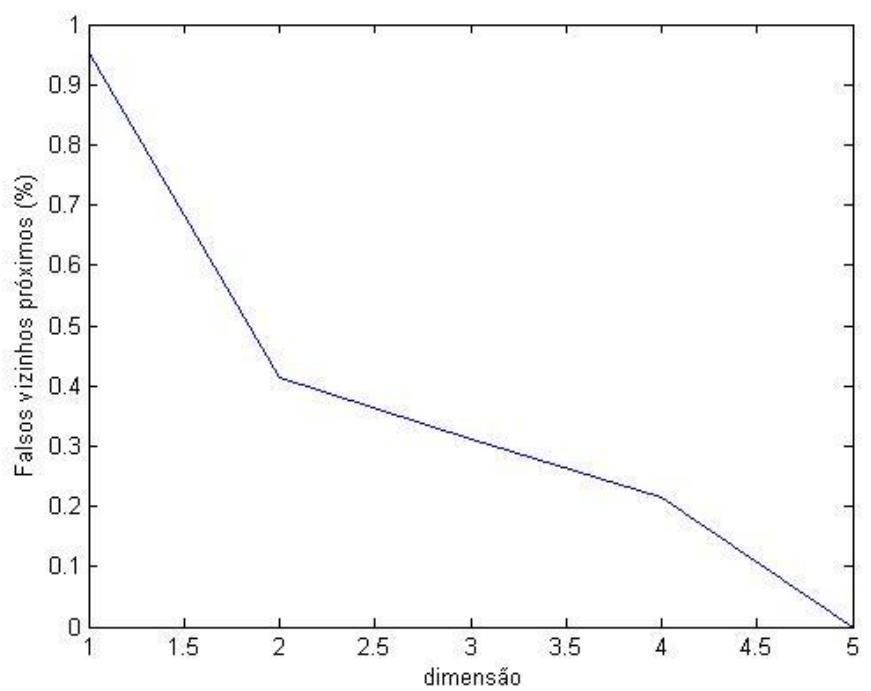

Figura 5. 65 - Porcentagem de falsos vizinhos para a série analisada em borracha na altura $\mathrm{Z}=306 \mathrm{~nm}$ com $m=5$. 


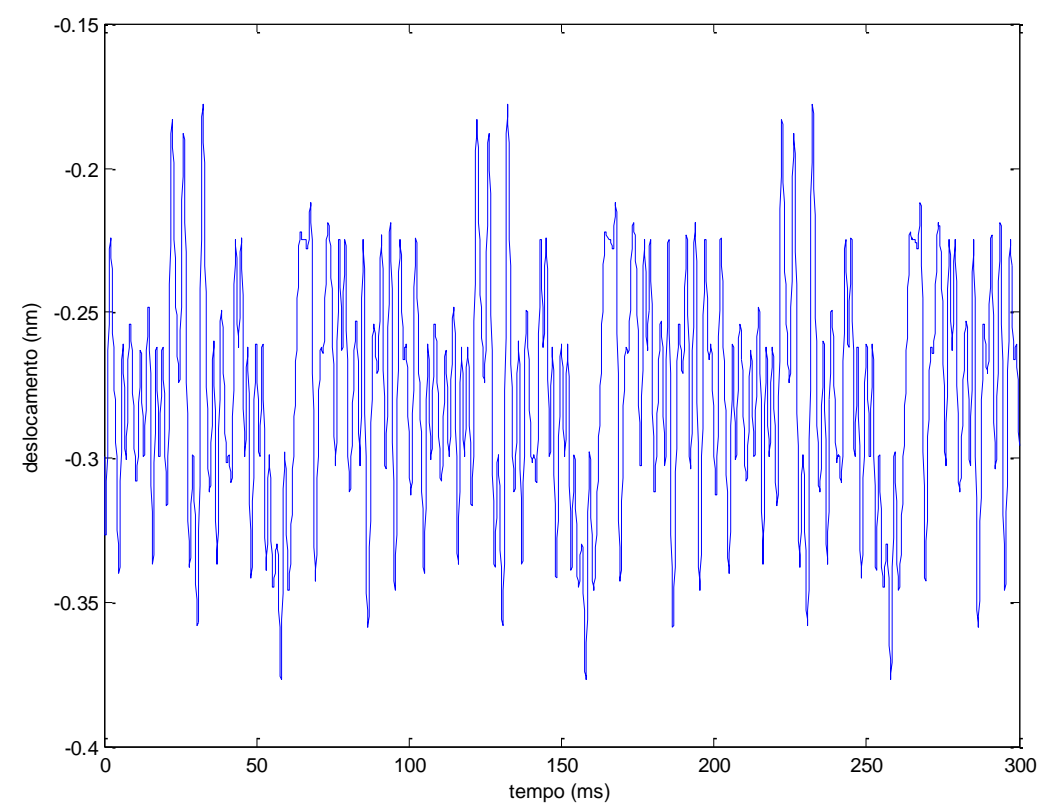

Figura 5. 66 - Deslocamento da microviga em z=306nm

O retrato de fase obtido pelo método das coordenadas defasadas em Fig 5.67 apresenta um movimento difícil de caracterizar em caótico ou semi-periódico. Já em Fig 5.68 a reconstrução do espaço de estados pelo método das derivadas mostra certos períodos de repetição. $\mathrm{O}$ resultado pode ser creditado a troca de amostra de safira por borracha mesmo em Fig. 5.69 configurando caos.

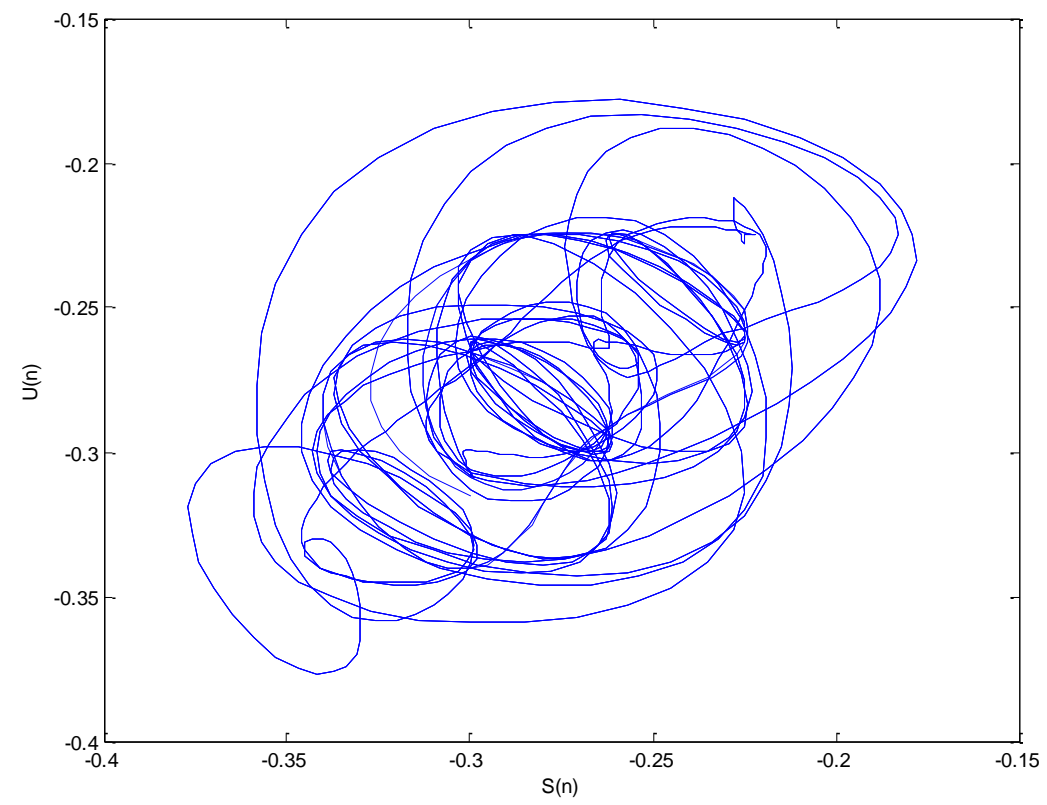

Figura 5. 67 - Retrato de fase da série temporal analisada em borracha com Z=306nm reconstruída com $m=5$ e $\tau=8$ 


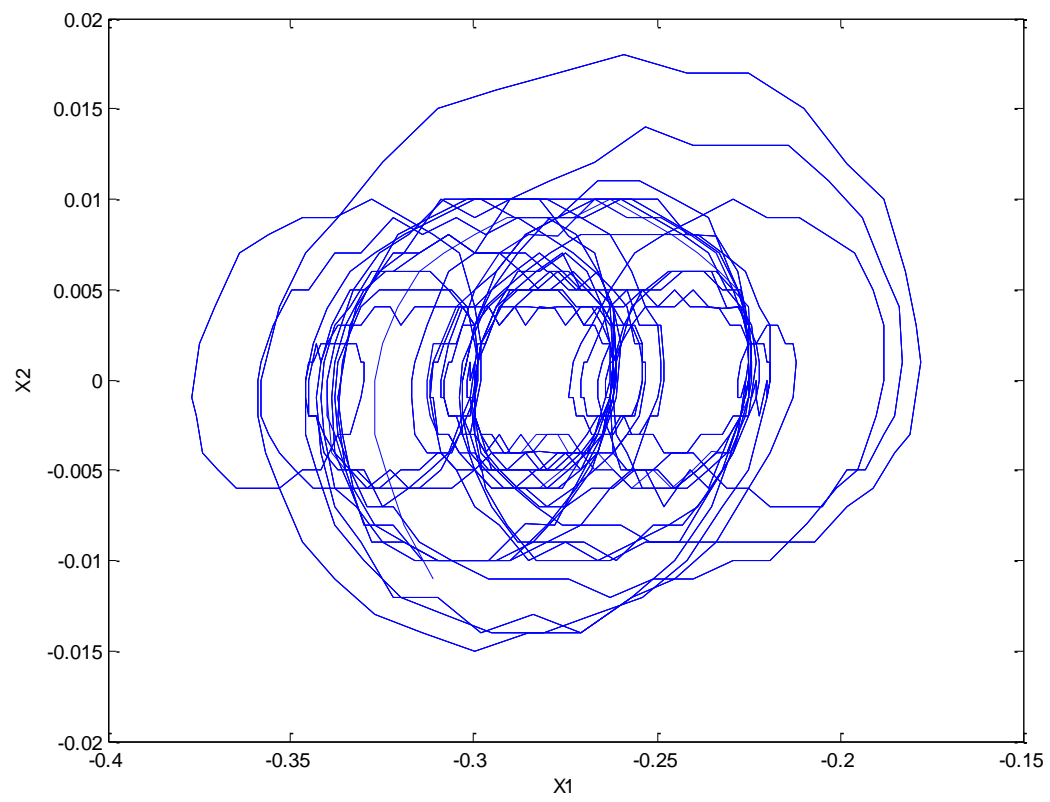

Figura 5. 68 - Reconstrução do espaço de estados pelo método das derivadas da série analisada em borracha com $\mathrm{Z}=306 \mathrm{~nm}$

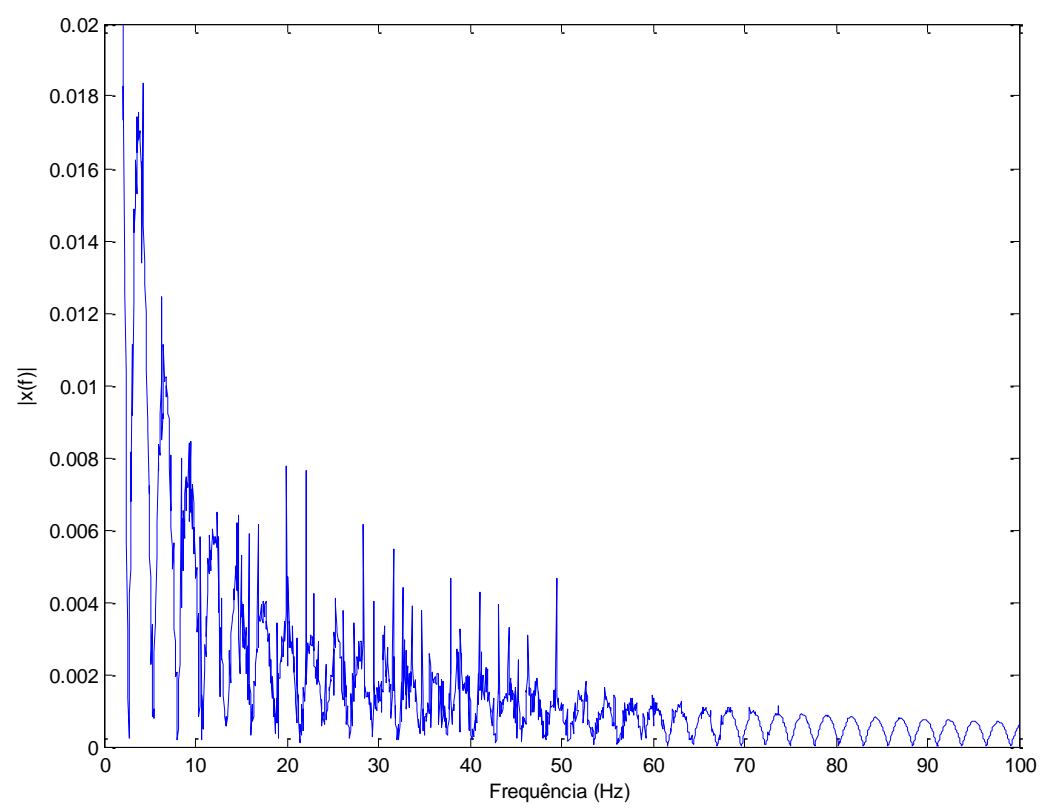

Figura 5. 69 - FFT da série produzida em borracha com Z=306nm

A seguinte análise foca a série produzida em borracha na altura $Z=382 \mathrm{~nm}$, cujo teste 0-1 indica o maior valor $K$. Calculando a informação mútua da série tem- se: 
Tabela 10 - Informação mútua da série $\mathrm{C}$ em z=382nm

\begin{tabular}{|c|c|}
\hline$\tau$ & Informação mútua \\
\hline 0 & 2.419753 \\
\hline 1 & 1.569458 \\
\hline 2 & 1.224137 \\
\hline 3 & 0.9802113 \\
\hline 4 & 0.7721651 \\
\hline 5 & 0.6519414 \\
\hline 6 & 0.5390858 \\
\hline 7 & 0.4351547 \\
\hline 8 & 0.3673408 \\
\hline 9 & 0.3675747 \\
\hline 10 & 0.3447284 \\
\hline 11 & 0.2912996 \\
\hline 12 & 0.2799495 \\
\hline 13 & 0.2891001 \\
\hline 14 & 0.3126904 \\
\hline 15 & 0.3071606 \\
\hline 16 & 0.2835538 \\
\hline 17 & 0.2715818 \\
\hline 18 & 0.2488873 \\
\hline 19 & 0.3034705 \\
\hline 20 & 0.2839025 \\
\hline
\end{tabular}

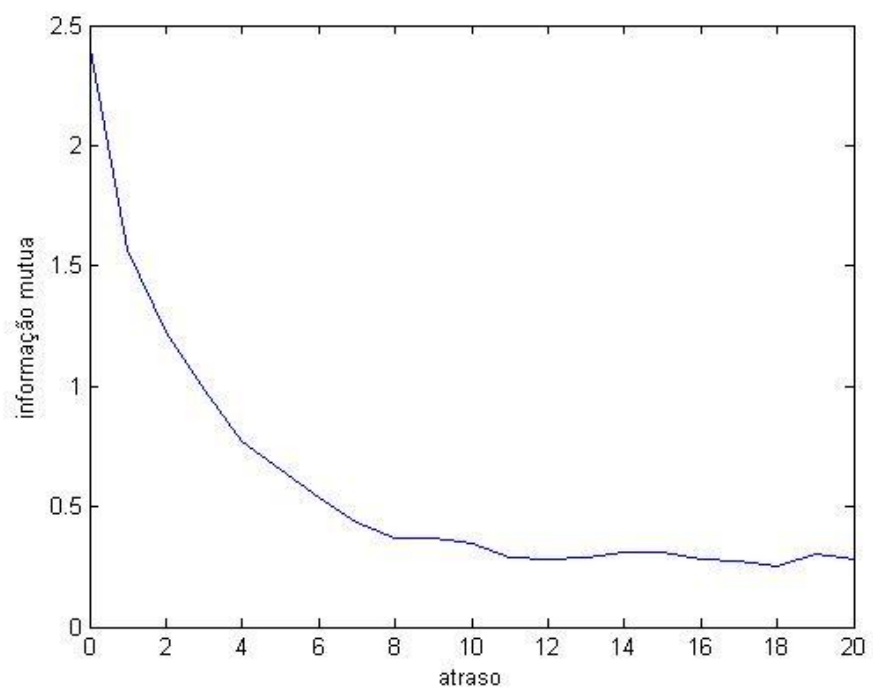

Figura 5. 70 - Informação mútua da série temporal analisada em borracha na altura $Z=382.2$ nm com mínimo local em $\tau=10$ 


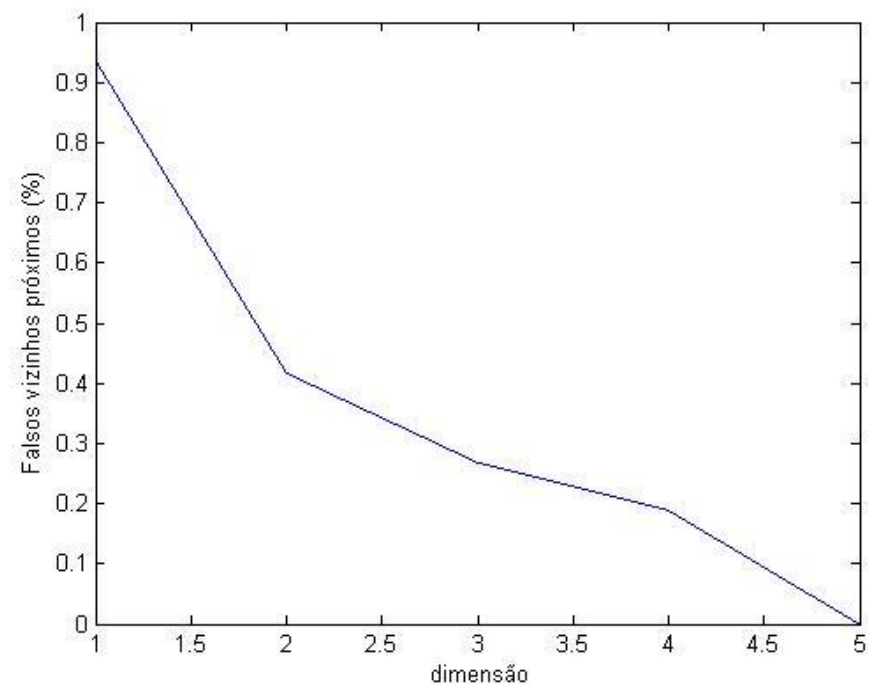

Figura 5. 71 - Porcentagem de falsos vizinhos para a série analisada em borracha na altura $\mathrm{Z}=382.2 \mathrm{~nm}$ com $m=5$.

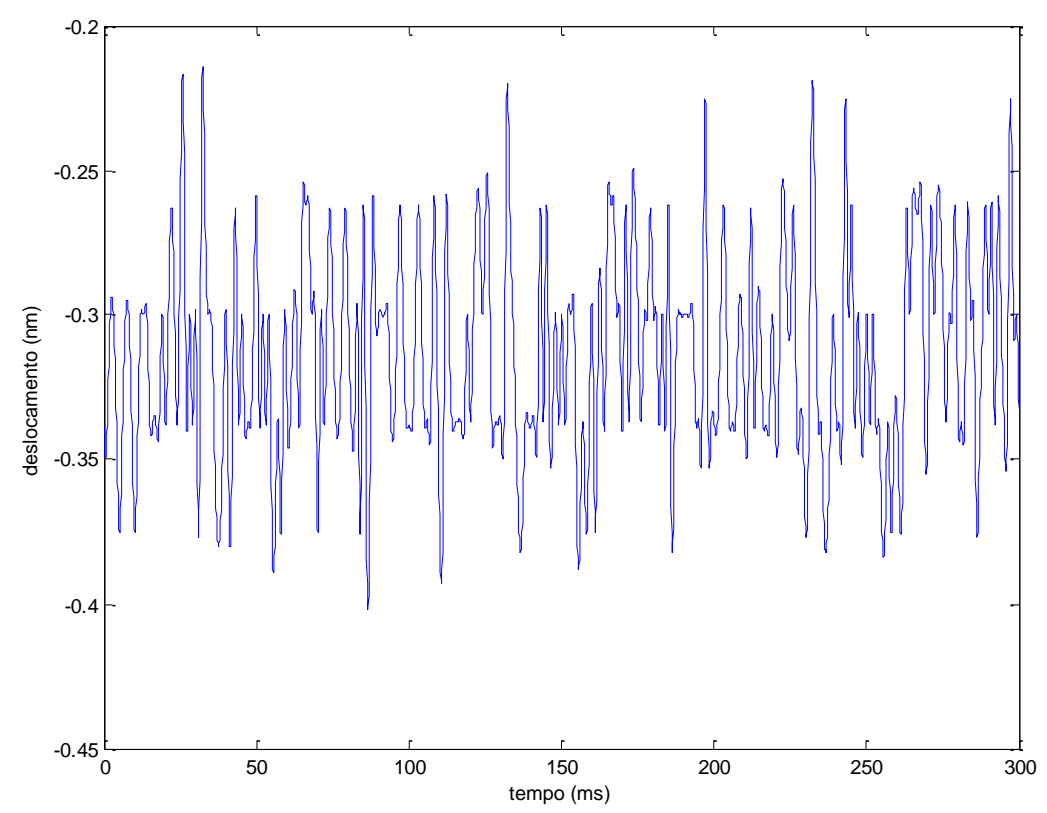

Figura 5. 72 - Deslocamento da microviga em z=382.2nm 


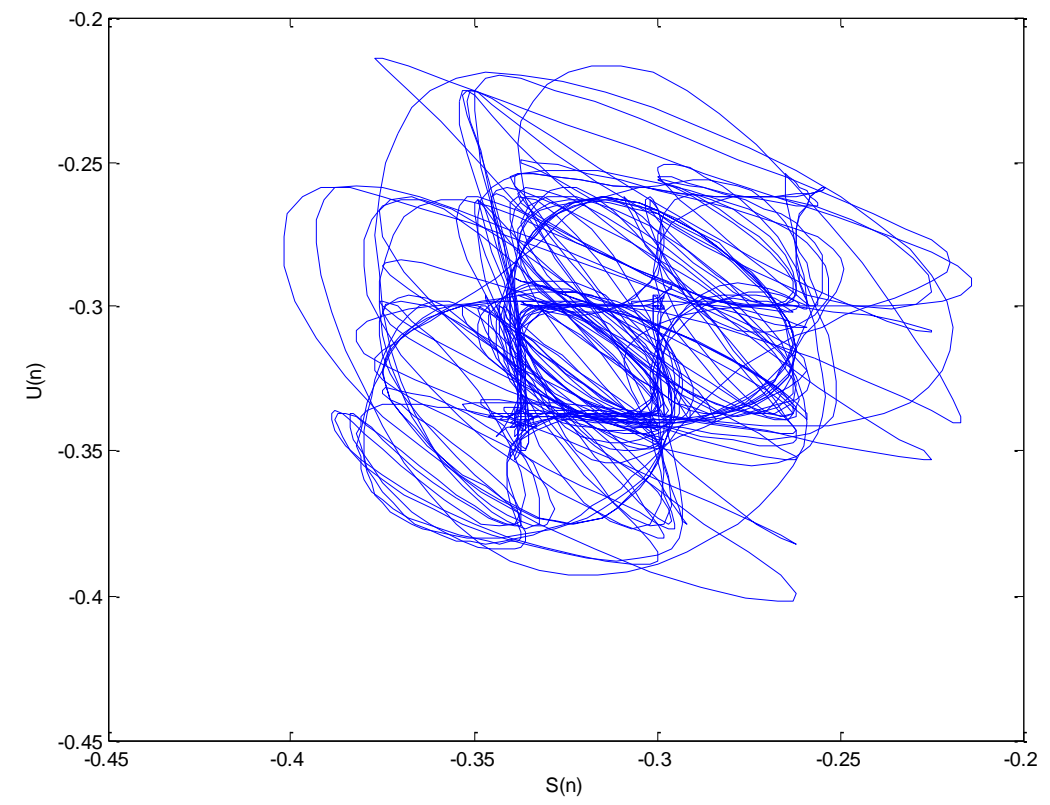

Figura 5. 73 - Retrato de fase da série temporal em borracha com Z=382.2 $\mathrm{nm}$ reconstruída com $m=5$ e $\tau=13$

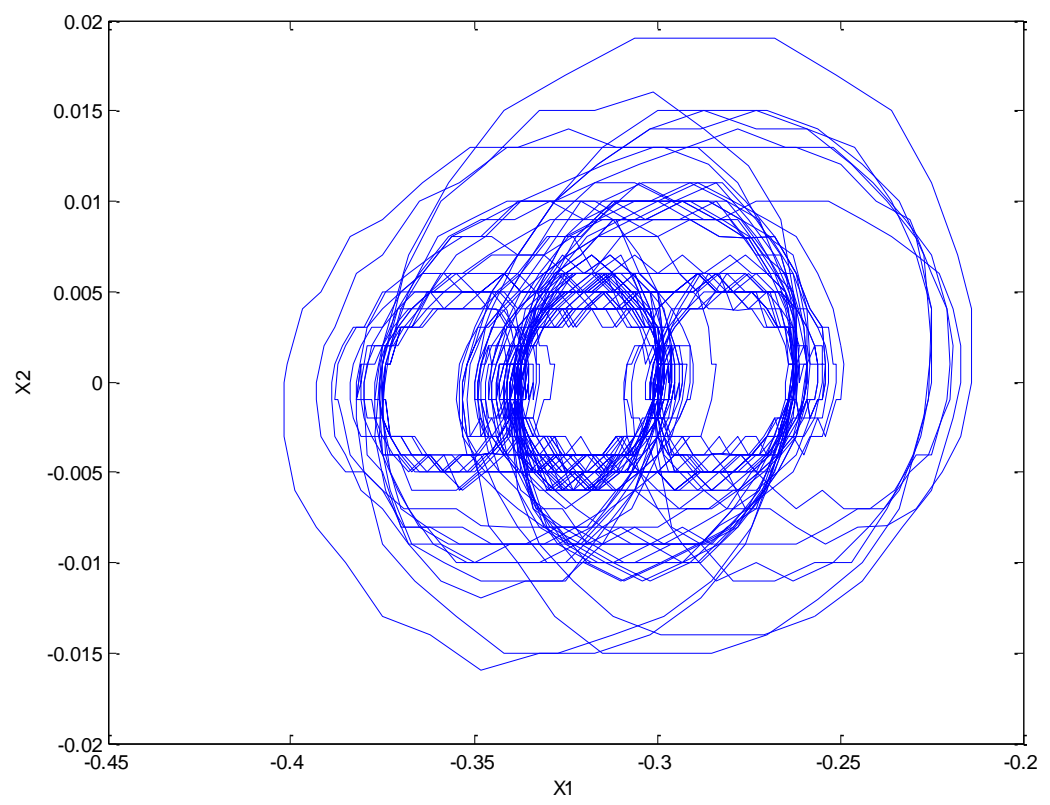

Figura 5. 74 - Retrato de fase reconstruída com série analisada em borracha em Z=382.2nm pelo método das derivadas 


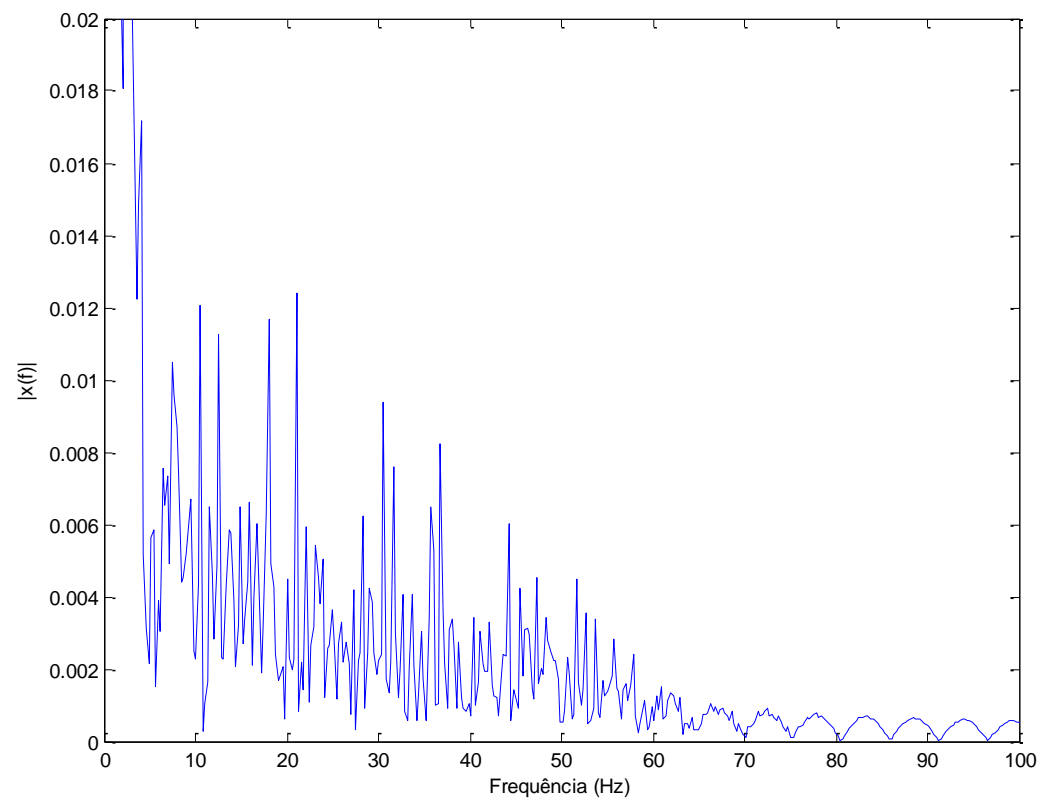

Figura 5. 75 - FFT da série temporal analisada em borracha com Z=382.2nm.

\subsubsection{SÉRIE TEMPORAL D}

O experimento D foi feito com safira em nitrogênio, scan size zero, constante elástica de $42 \mathrm{~N} / \mathrm{m}$, ponteira triangular, sens. amp/sens de $100 \mathrm{~nm} / \mathrm{V}$, amplitude setpoint de $170,82 \mathrm{mV}$, drive amplitude de $50.96 \mathrm{mV}$ e temperatura de $22.4^{\circ} \mathrm{C}$

Tabela 11 - Tabela com teste $0-1$ e escala indexada aplicada na série 'd'

\begin{tabular}{c|c|c|c|c|c|c|c|c}
\hline $\mathbf{Z}$ & $\mathbf{K}$ & $\begin{array}{c}\text { LACA } \\
\text { INDE } \\
\text { XADA }\end{array}$ & $\mathbf{Z}$ & $\mathbf{K}$ & $\begin{array}{c}\text { LA } \\
\text { INDE } \\
\text { XADA }\end{array}$ & $\mathbf{Z}$ & $\mathbf{K}$ & $\begin{array}{c}\text { ESCA } \\
\text { INDE } \\
\text { XADA }\end{array}$ \\
\hline 2 & 0,99682 & 0,99891 & 249 & 0,9815 & 0,99815 & 496 & 0,96957 & 0,99823 \\
21 & 0,9972 & 0,9984 & 268 & 0,97967 & 0,99815 & 515 & 0,97054 & 0,99823 \\
40 & 0,99778 & 0,99898 & 287 & 0,98254 & 0,99815 & 534 & 0,98674 & 0,99822 \\
59 & 0,99705 & 0,99842 & 306 & 0,98429 & 0,99815 & 553 & 0,73824 & 0,99781 \\
78 & 0,98611 & 0,99836 & 325 & 0,99399 & 0,99814 & 572 & 0,94653 & 0,99806 \\
97 & 0,98917 & 0,99836 & 344 & 0,99417 & 0,99807 & 591 & 0,93798 & 0,99806 \\
116 & 0,98781 & 0,99836 & 363 & 0,99306 & 0,99821 & 610 & 0,92707 & 0,99806 \\
135 & 0,98864 & 0,99836 & 382 & 0,99556 & 0,99809 & 629 & 0,91576 & 0,99803 \\
154 & 0,9871 & 0,99836 & 401 & 0,99328 & 0,99827 & 648 & 0,97796 & 0,99811 \\
173 & 0,98862 & 0,99836 & 420 & 0,99054 & 0,99828 & & & \\
192 & 0,98533 & 0,99836 & 439 & 0,96528 & 0,99823 & & &
\end{tabular}




\begin{tabular}{l|c|c|c|c|c|l|l|l}
211 & 0,9932 & 0,99843 & 458 & 0,94935 & 0,99823 & & & \\
230 & 0,99191 & 0,99826 & 477 & 0,95068 & 0,99823 & & & \\
\hline
\end{tabular}

Esse experimento mostra uma certa região próxima do movimento periódico comprovada pelo método do teste $0-1$ na fig. 5.78. Quando $\mathrm{z}=553 \mathrm{~nm}$ coincidentemente os testes avaliam com o menor valor caótico. Para a escala indexada da fig.5.79 o valor ainda apresenta caos e para o teste $0-1$ o resultado mostra a série com caos fraco.

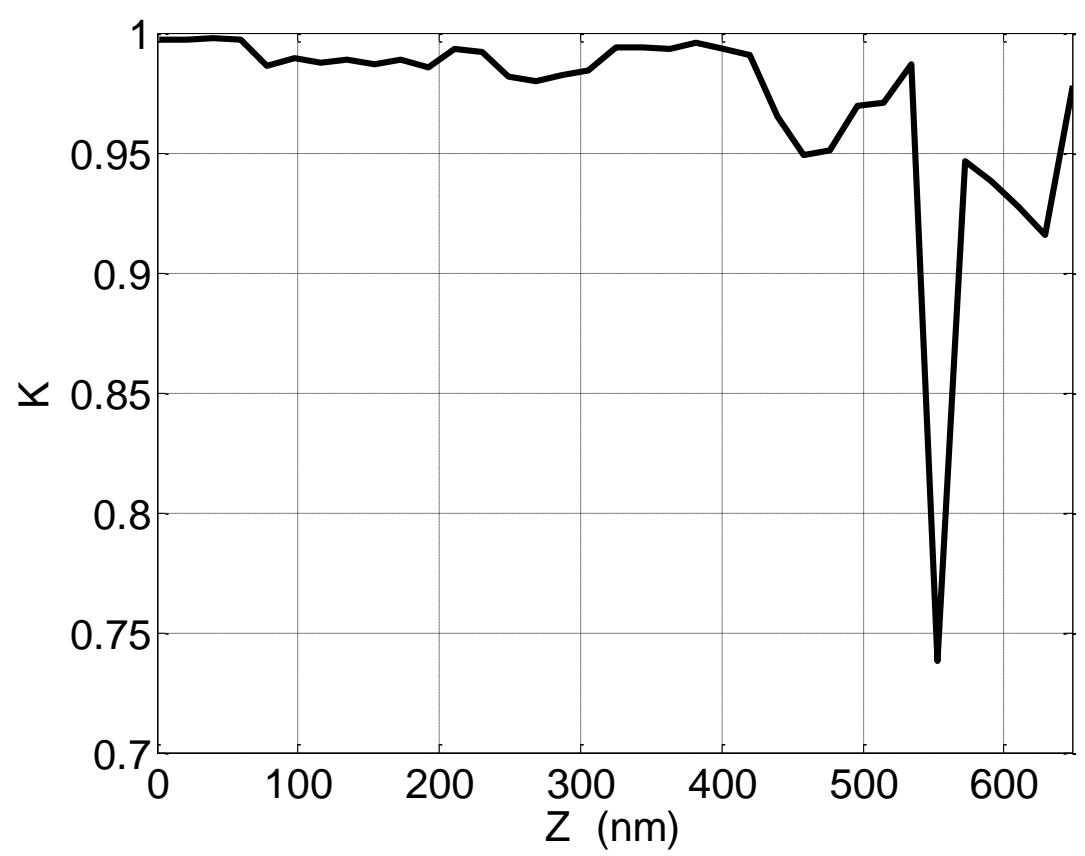

Figura 5. 76 - Variação de $K$ do teste 0 -1 para série 'd'

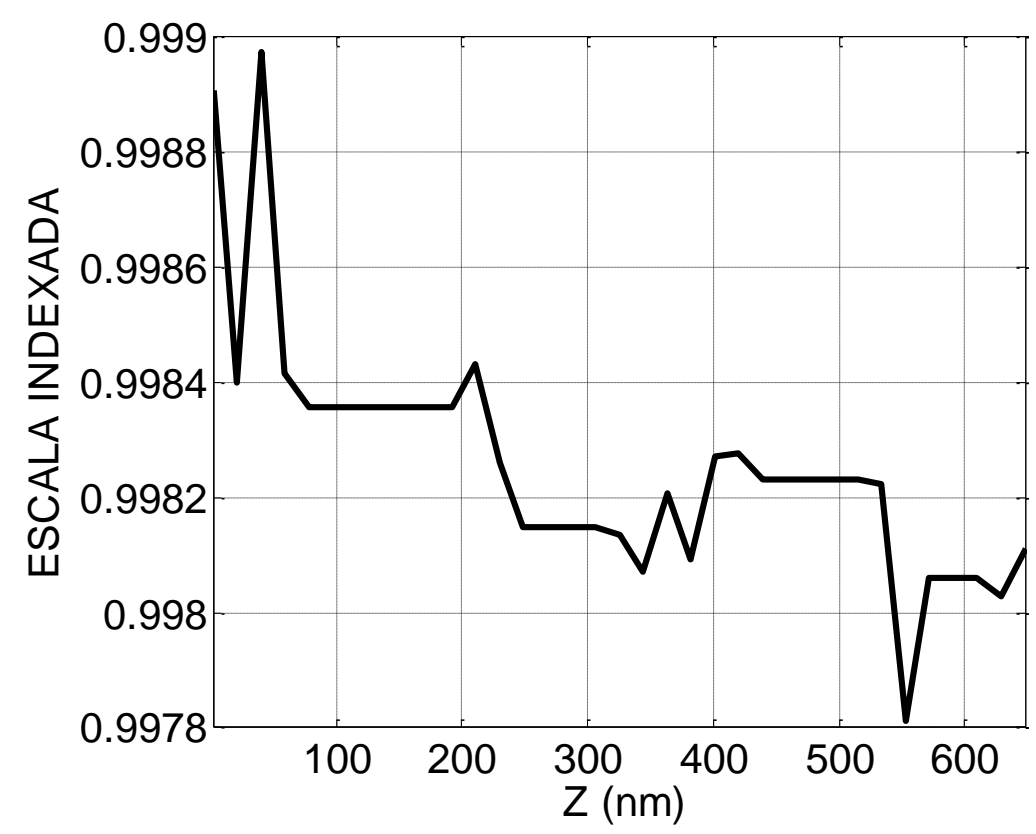

Figura 5. 77 - Teste da escala indexada para a série 'd' 
Para a série de safira no nitrogênio com valor de $Z=40 \mathrm{~nm}$ o teste 0 -1 indica o valor $\mathrm{K}$ máximo. Calcula-se a informação mútua, falsos vizinhos próximos para a reconstrução do espaço de estados.

Tabela 12- Informação mútua para série D em z=40nm

\begin{tabular}{|c|c|}
\hline$\tau$ & Informação mútua \\
\hline 0 & 2.297456 \\
\hline 1 & 1.437867 \\
\hline 2 & 1.146116 \\
\hline 3 & 0.8814681 \\
\hline 4 & 0.746068 \\
\hline 5 & 0.6058546 \\
\hline 6 & 0.5095762 \\
\hline 7 & 0.453134 \\
\hline 8 & 0.3827035 \\
\hline 9 & 0.3764624 \\
\hline 10 & 0.3578148 \\
\hline 11 & 0.3445487 \\
\hline 12 & 0.3197861 \\
\hline 13 & 0.34574 \\
\hline 14 & 0.3089823 \\
\hline 15 & 0.2902356 \\
\hline 16 & 0.2658315 \\
\hline 17 & 0.2936056 \\
\hline 18 & 0.2974362 \\
\hline 19 & 0.2985096 \\
\hline 20 & 0.306104 \\
\hline
\end{tabular}




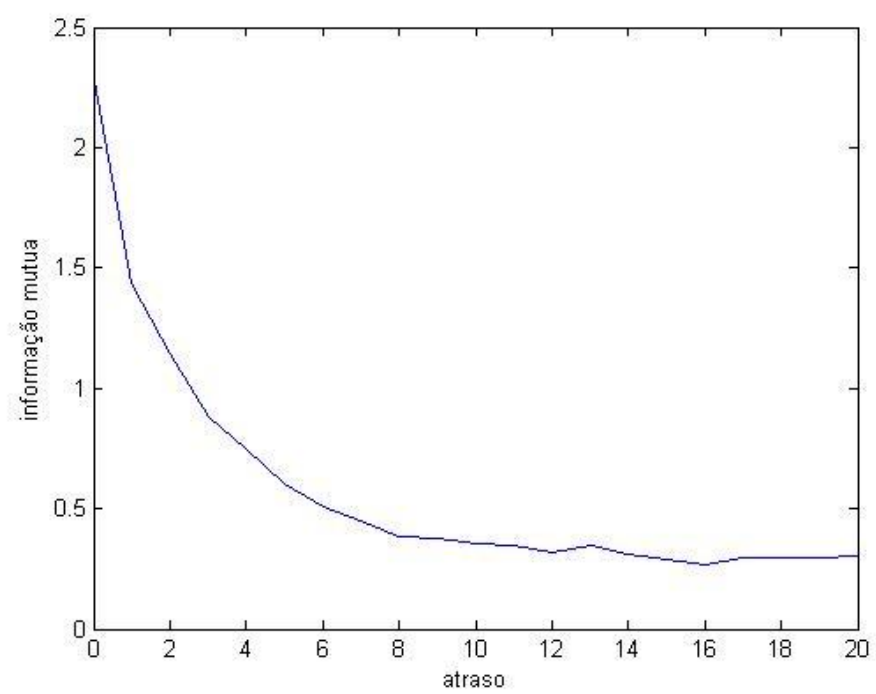

Figura 5. 78 - Informação mútua da série temporal analisada em safira no nitrogênio na altura $\mathrm{Z}=40 \mathrm{~nm}$ com mínimo local em $\tau=12$

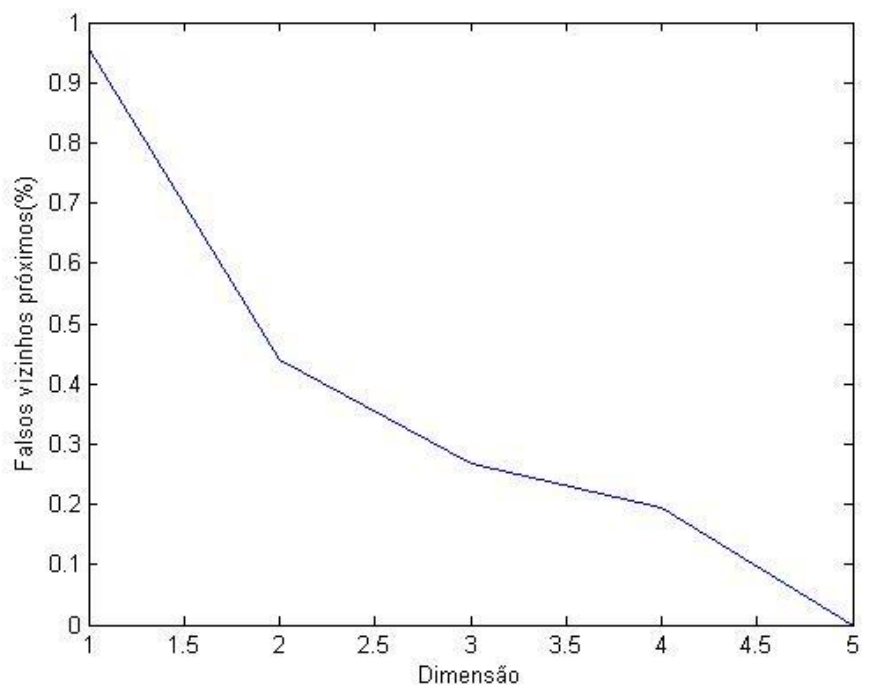

Figura 5. 79 - Porcentagem de falsos vizinhos para a série analisada na safira com nitrogênio na altura $Z=40 \mathrm{~nm}$ com $m=5$. 


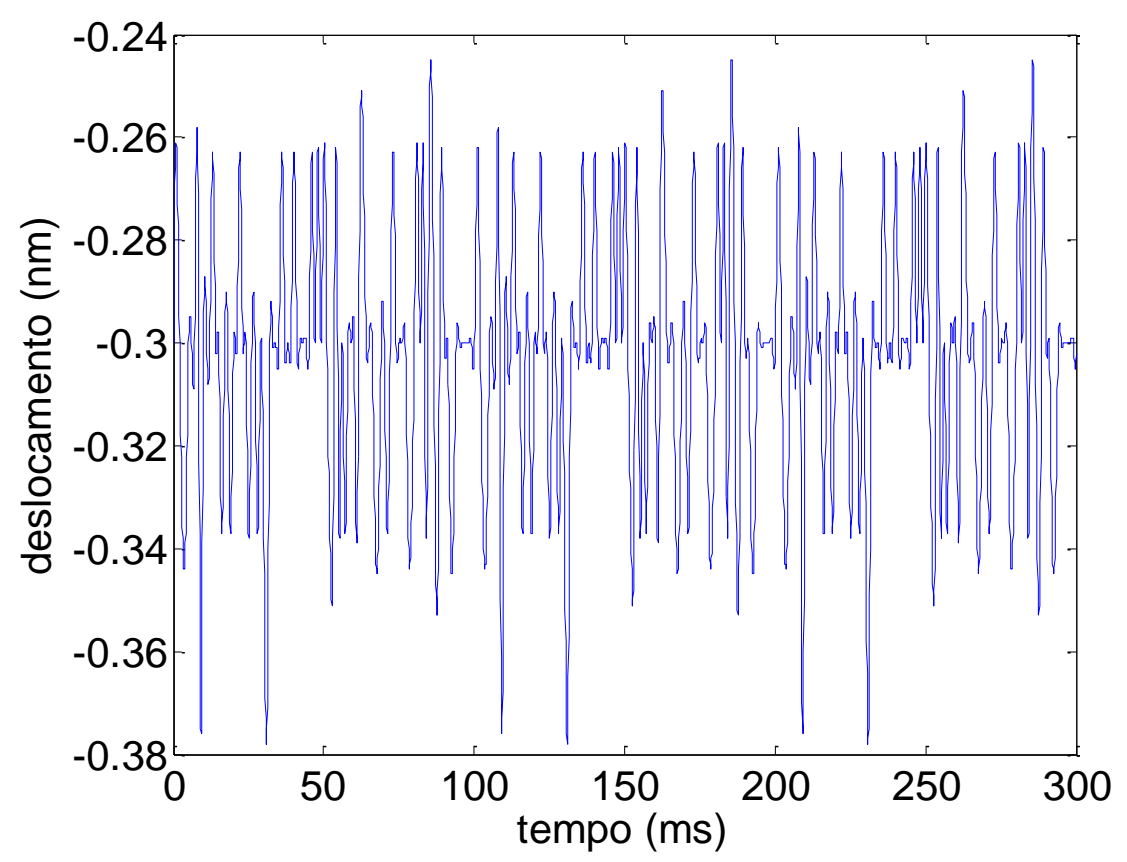

Figura 5. $80-$ Deslocamento da microviga em $\mathrm{z}=40 \mathrm{~nm}$

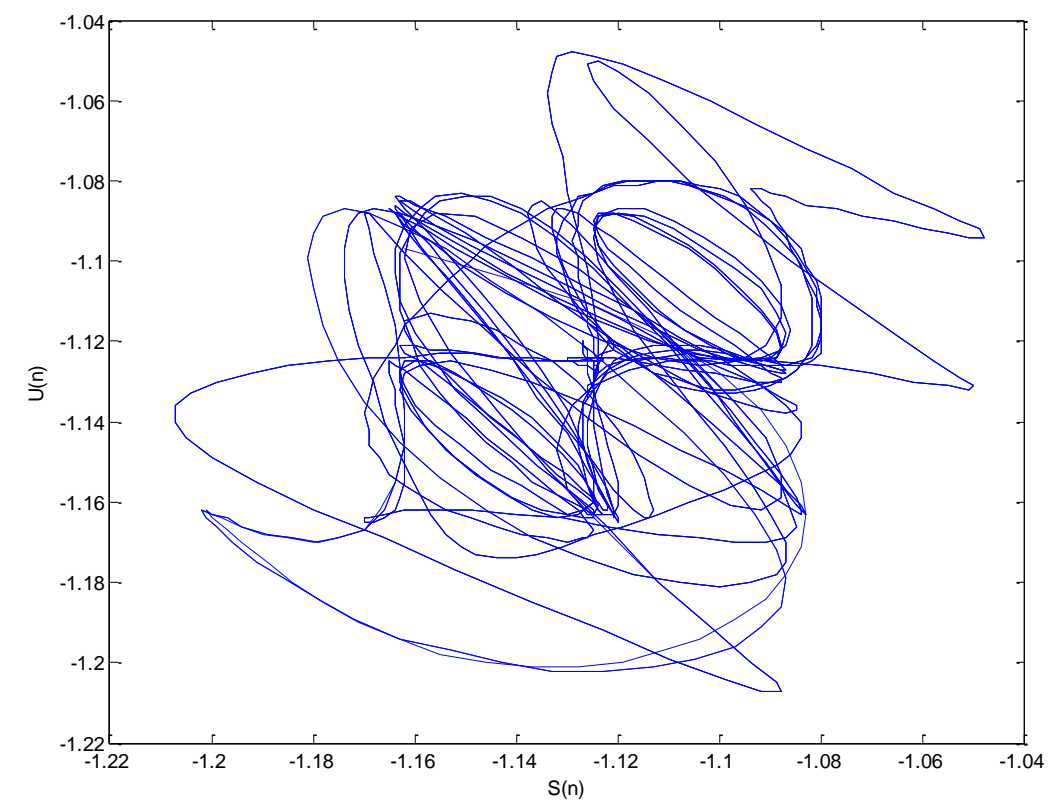

Figura 5. 81- Retrato de fase da série temporal em safira com nitrogênio com $Z=40 \mathrm{~nm}$ reconstruída com $m=5$ e $\tau=12$ 


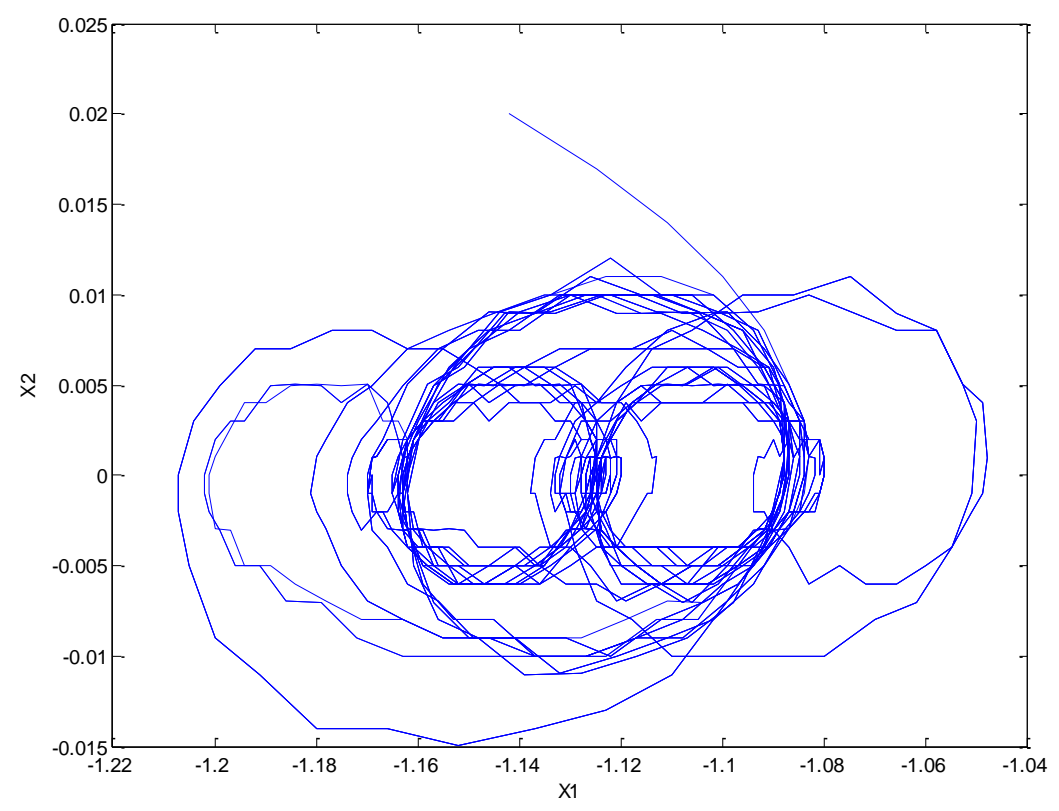

Figura 5. 82- Retrato de fase reconstruído da série analisada na safira em nitrogênio pelo método das derivadas com $\mathrm{Z}=40 \mathrm{~nm}$

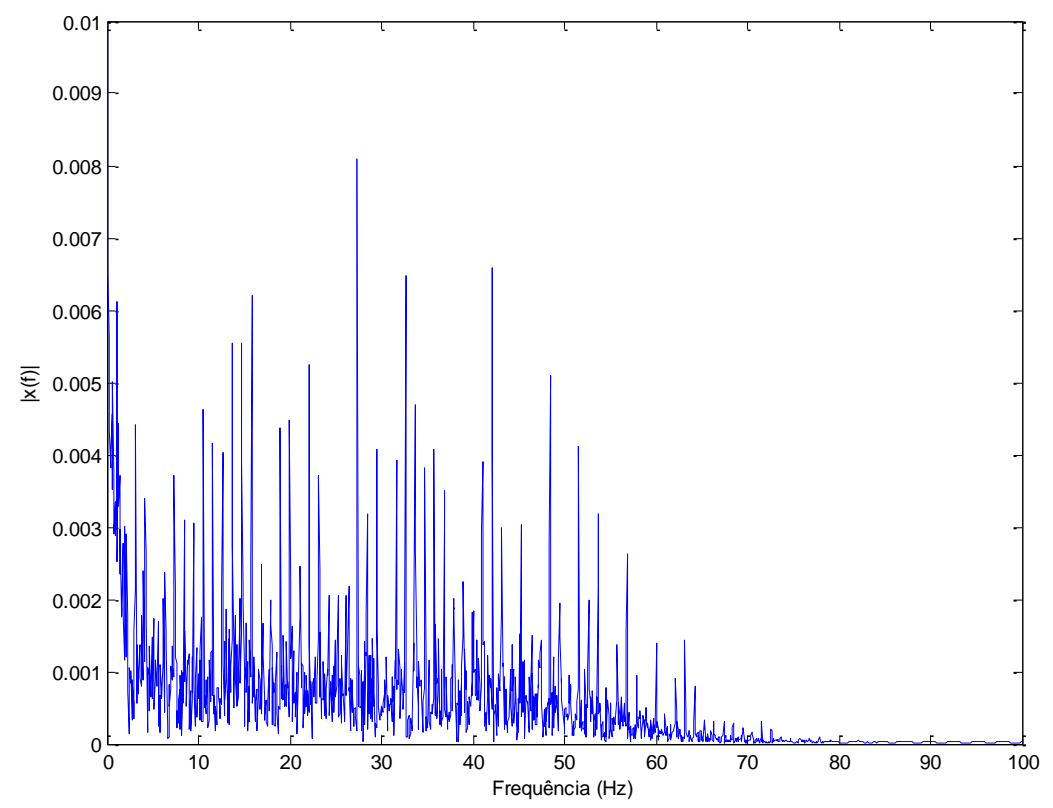

Figura 5. 83 - FFT da série temporal analisada em safira com nitrogênio com Z=40nm

Para a série $\mathrm{Z}=553 \mathrm{~nm}$ o teste $\mathrm{k}$ tem o valor mínimo. Aplicando a informação mútua tem-se:

Tabela 13- Informação mútua para série D em z=553nm

\begin{tabular}{|c|l|}
\hline$\tau$ & Informação mútua \\
\hline 0 & 2.34066700000000 \\
\hline
\end{tabular}




\begin{tabular}{|c|c|}
\hline 1 & 1.46440 \\
\hline 2 & 1.144606 \\
\hline 3 & 0.9173116 \\
\hline 4 & 0.7588881 \\
\hline 5 & 0.6689648 \\
\hline 6 & 0.538597 \\
\hline 7 & 0.4575753 \\
\hline 8 & 0.4058885 \\
\hline 9 & 0.3521007 \\
\hline 10 & 0.3770358 \\
\hline 11 & 0.3423804 \\
\hline 12 & 0.3045742 \\
\hline 13 & 0.3178831 \\
\hline 14 & 0.3239861 \\
\hline 15 & 0.3470465 \\
\hline 16 & 0.2853336 \\
\hline 17 & 0.2796128 \\
\hline 18 & 0.2336983 \\
\hline 19 & 0.2776948 \\
\hline 20 & 0.2599516 \\
\hline
\end{tabular}

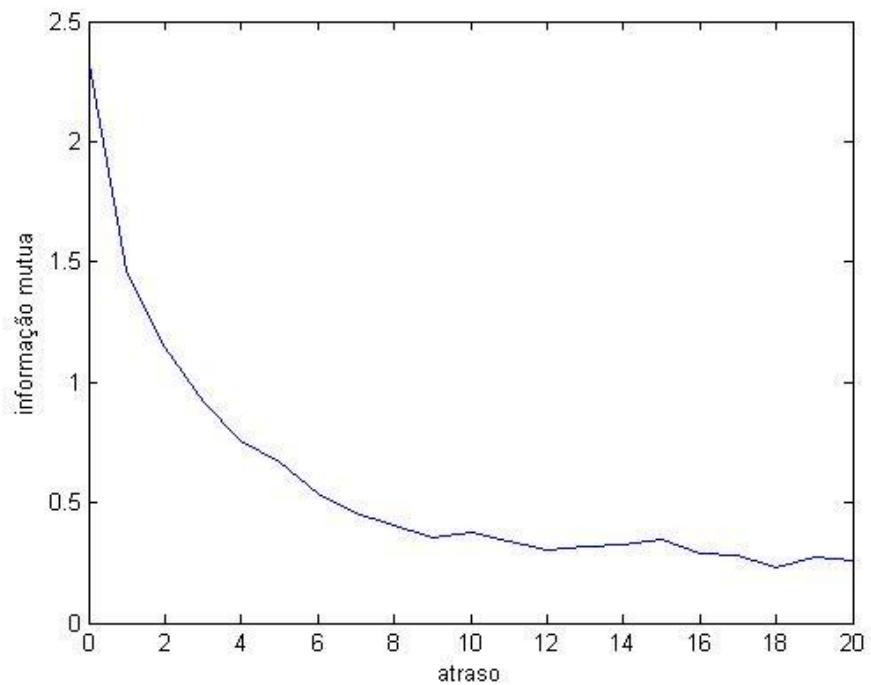

Figura 5. 84 - Informação mútua da série temporal analisada em safira no nitrogênio na altura $\mathrm{Z}=553 \mathrm{~nm}$ com mínimo local em $\tau=9$ 


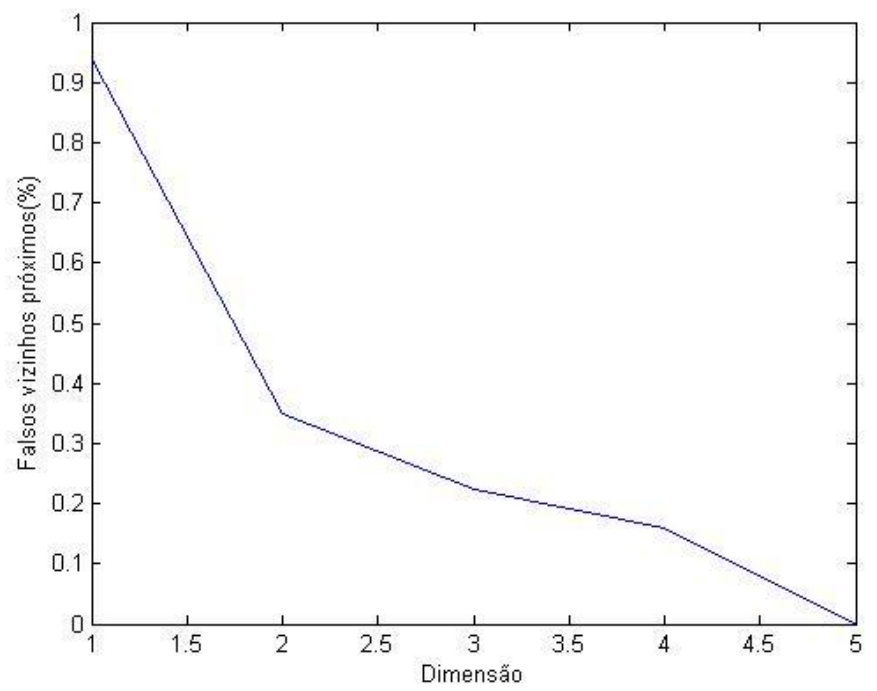

Figura 5. 85 - Dimensão $m=5$ calculado pelo algoritmo de falso vizinhos próximos para a série analisada em safira no nitrogênio com $Z=553 \mathrm{~nm}$

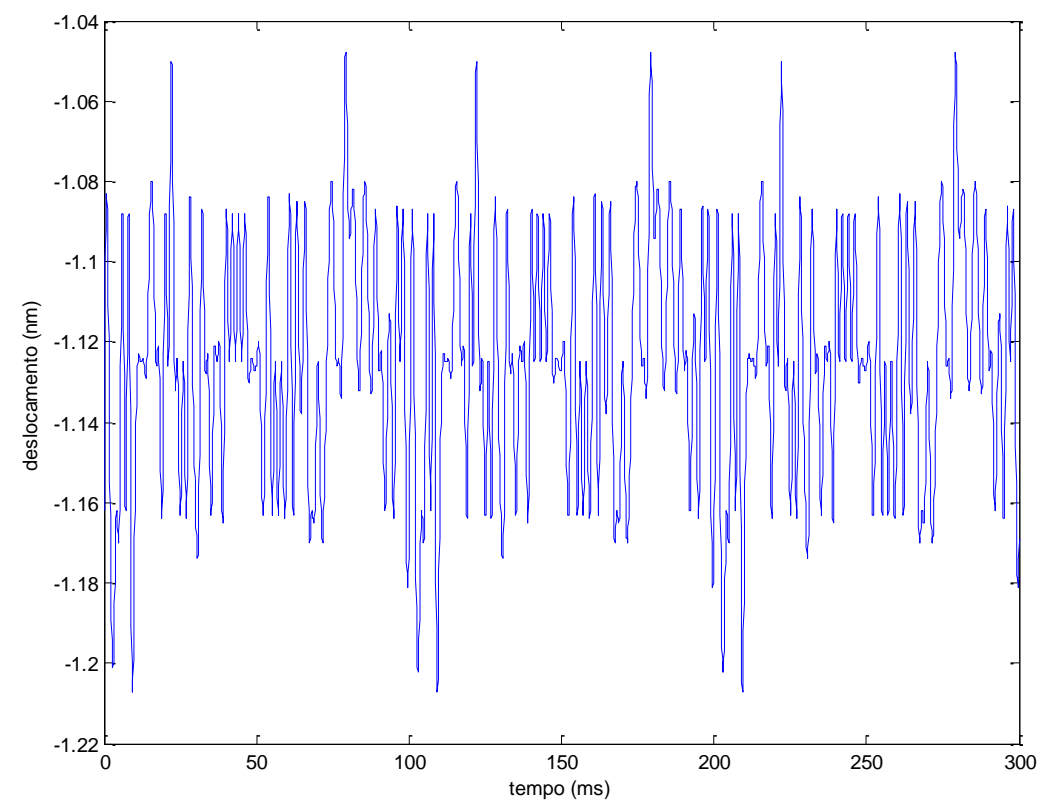

Figura 5. 86- Deslocamento da microviga em z=553nm

$\mathrm{O}$ retrato de fase obtido pelas coordenadas defasadas na fig 5.87 mostra uma certa periodicidade assim como na fig. 5.88 que mostra o período 2 . 


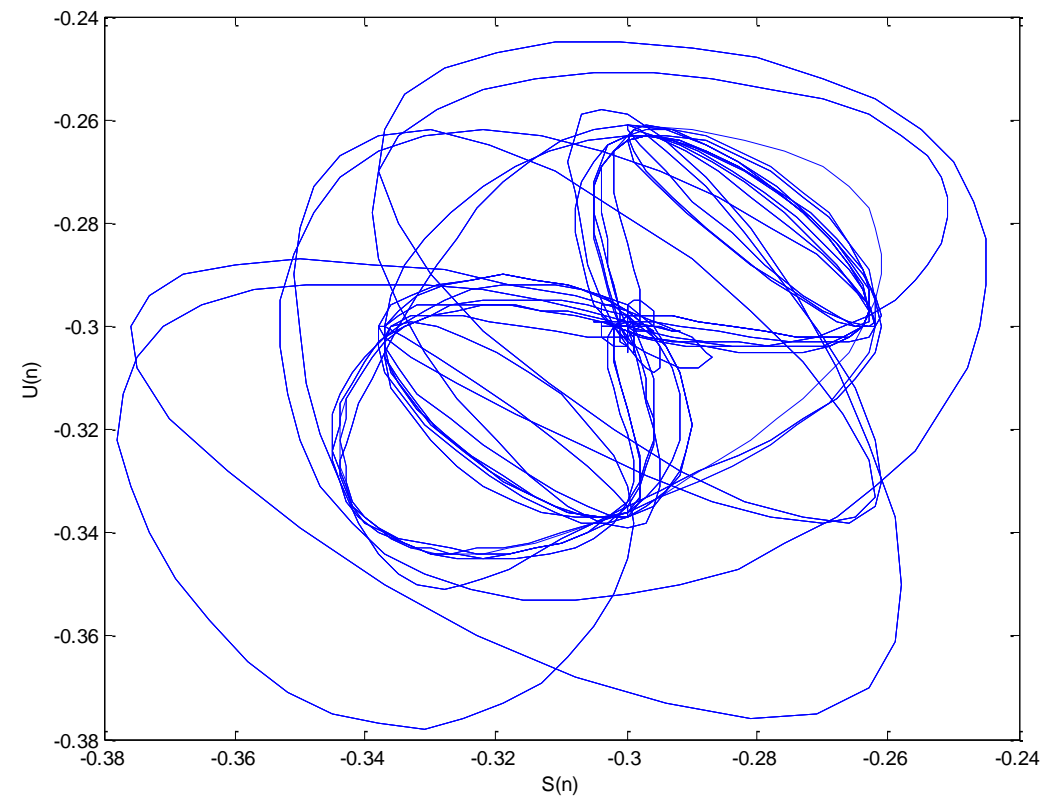

Figura 5. 87 - Retrato de fase da série temporal $\mathrm{z}=553 \mathrm{~nm}$ em safira no nitrogênio reconstruída com $m=5$ e $\tau=9$

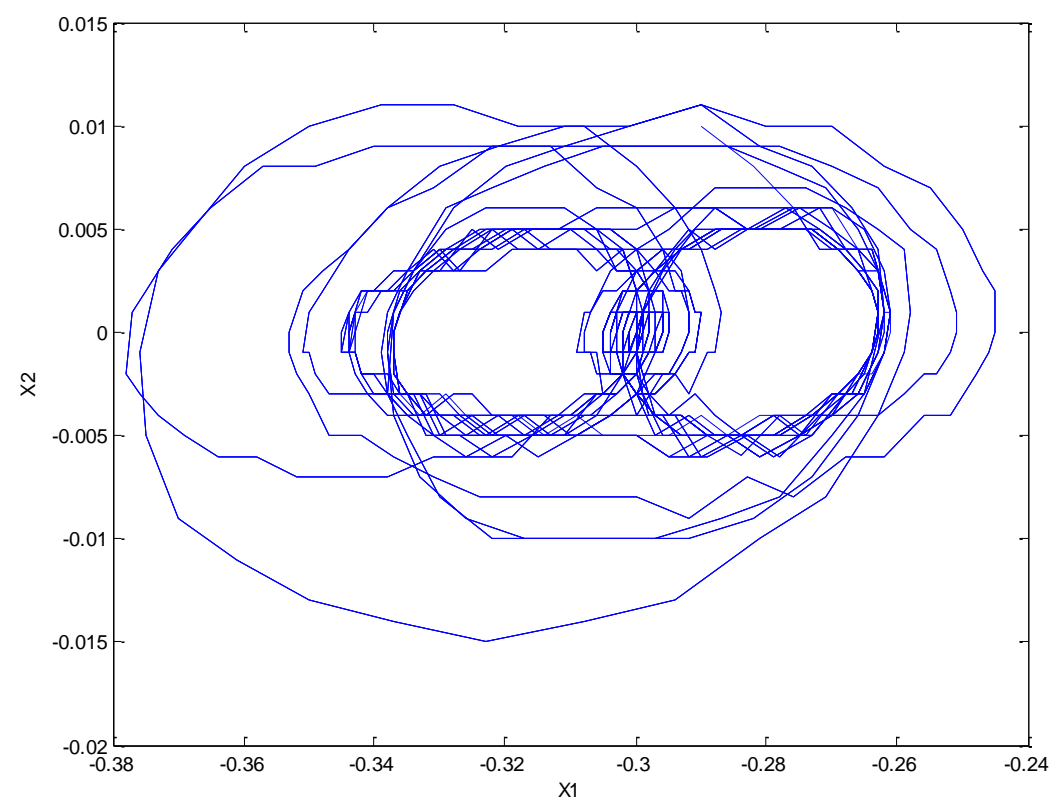

Figura 5. 88 - Reconstrução de espaço de estados pelo método das derivadas na série de safira no nitrogênio em $\mathrm{Z}=553 \mathrm{~nm}$ 


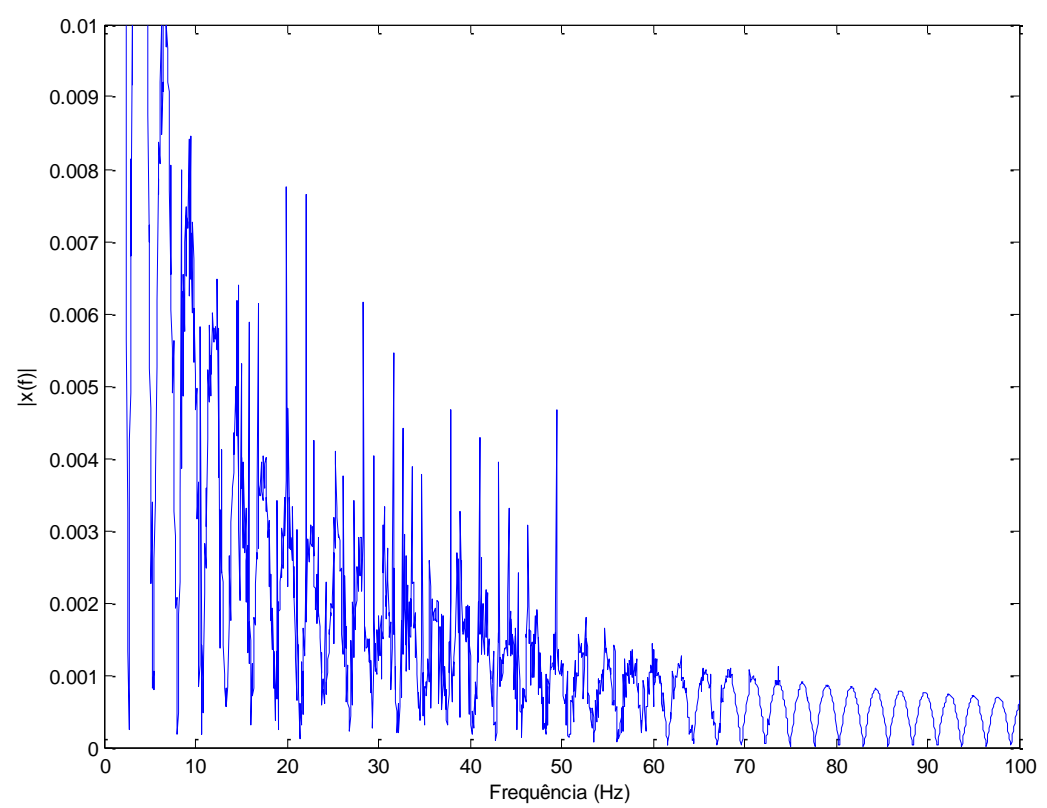

Figura 5. 89 - FFT para série em safira no nitrogênio com $\mathrm{Z}=553 \mathrm{~nm}$

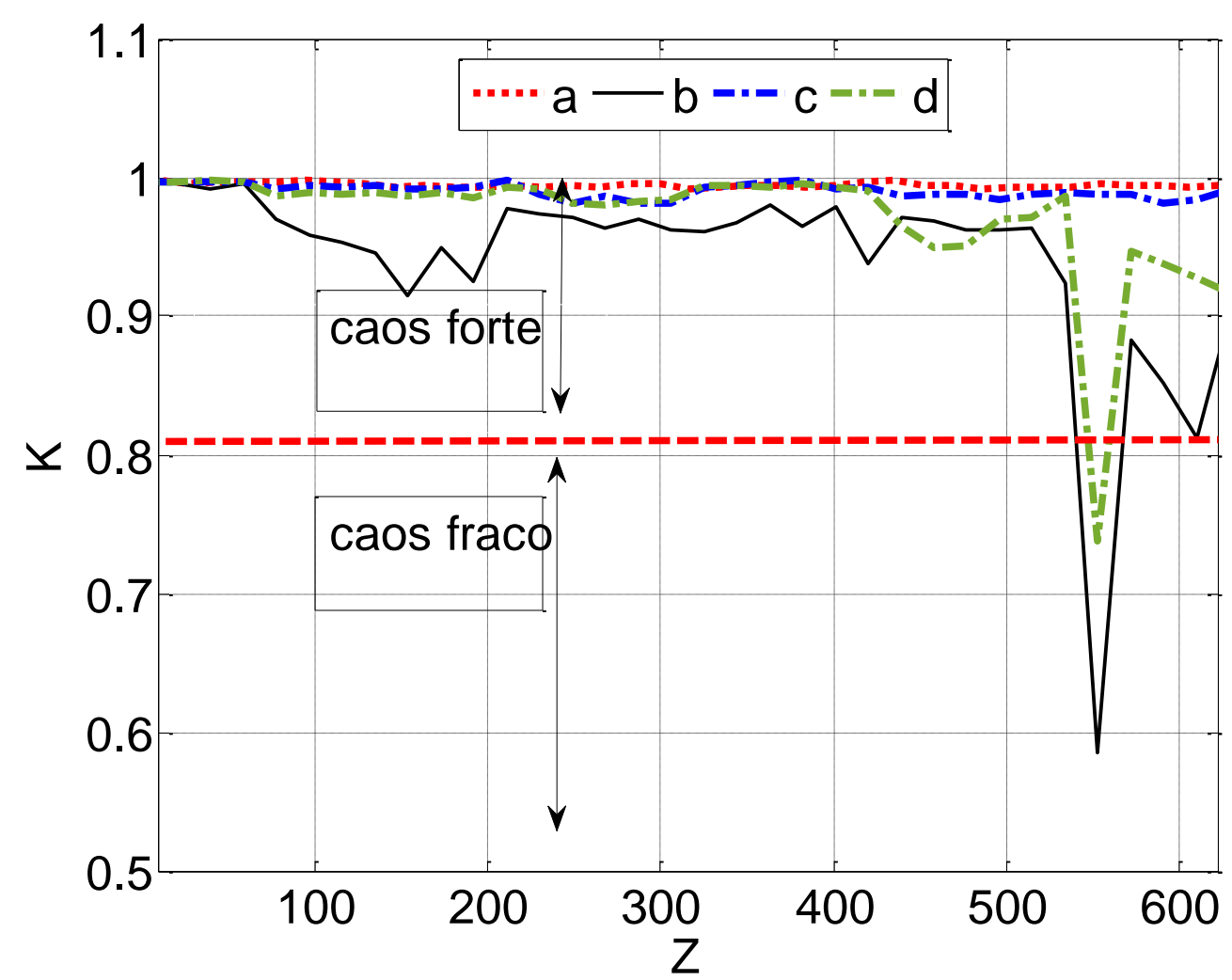

Figura 5. 90 - Gráfico de testes 0-1 para série 'a', 'b', 'c', e'd'

O teste mostra a série temporal 'a', 'b', 'c', e'd' sendo analisada de acordo com altura entre ponta e amostra variando entre $0 \mathrm{~nm}$ e $620 \mathrm{~nm}$, com a sendo a série temporal em safira, b a série temporal em safira com tip triângular, c a série em borracha, e $\mathbf{d}$ sendo a 
série em safira com nitrogênio. De uma maneira geral todas as séries se apresentam na faixa em que se denomina caos forte, excepcionalmente para o valor de $\mathrm{z}=553 \mathrm{~nm}$ a série $\mathbf{d}$ e $\mathbf{b}$ resultam em caos fraco pelo teste $0-1$. Este teste demonstra a dificuldade de se modelar um oscilador AFM. Quanto mais longe da amostra ( $\mathrm{Z}$ maior) a dinâmica caótica da microviga é menor se comparado a um $\mathrm{Z}$ pequeno, mesmo assim não deixando de ser caos forte, pois quanto mais perto da amostra, a microviga interage com as forças intermoleculares e de capilaridade com maior intensidade, o que reflete na sua dinâmica. Em apenas uma posição, duas séries mostraram se com caos fraco. Existem algumas variações no coeficiente K, mas em geral, é normalmente perto de 1, o que mostra um elevado grau de caos. Interessante notar que quando se muda a ponta, os resultados foram bastante diferentes (série 'a' e 'b'), enquanto em 'b' e 'c' mudaram se as amostras e manteve-se a ponta, ocorrendo resultados contrários, e comparando 'a' e 'd' , cuja única diferença foi ter colocado gás nitrogênio, os resultados mostraram que houveram uma pequena região menos caótica.

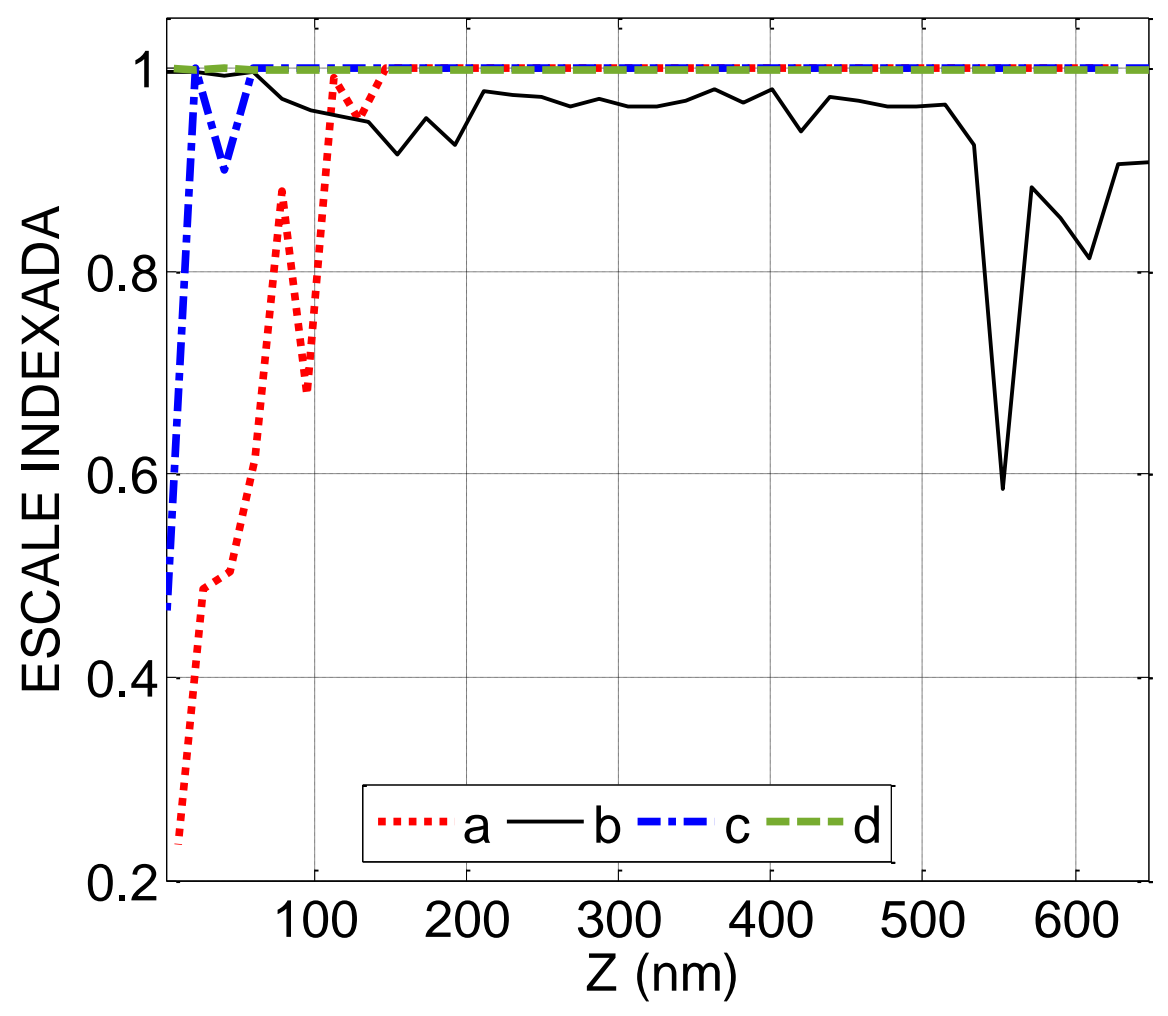

Figura 5. 91 - Teste escala indexada com as séries 'a', 'b', 'c' e 'd'.

Os resultados da escala indexada mostram coerência com o teste $0-1$, quando atestam o movimento caótico das séries. Nas séries a e c entre a altura $Z=0$ e $Z=30 \mathrm{~nm}$, que mostram 
uma faixa inferior a 0.5 na escala indexada, o que representa um movimento caótico mas menor que as alturas maiores que $\mathrm{z}=30 \mathrm{~nm}$. 


\section{CAPÍTULO 6}

\section{IDENTIFICAÇÃO DE SISTEMAS}

\section{POR ESPAÇO DE ESTADOS}

Alguns experimentos produzidos no laboratório de física da USP - São Carlos são analisados e identificados nesta seção. A não linearidade das séries temporais do microscópio de força atômica, garantida nos testes 0-1 e escala indexada, não garante que um processo não linear consiga identificar o sistema dinâmico.

Para a primeira série identificada foi selecionada a série com $\mathrm{z}=537,2 \mathrm{~nm}$ no modo não contato em ar, na amostra de superfície de Safira, constante elástica da microviga de 42 $N / m$, ponteira padrão retangular (standard), Sens. amp/sens de $100 \mathrm{~nm} / V$, amplitude setpoint de $178.85 \mathrm{mV}$, drive setpoint de $48.52 \mathrm{mV}$ e temperatura do laboratório de $22.1^{\circ} \mathrm{C}$, com entrada harmônica $u=[0.04 ;-0.04 ; 0.04 ;-0.04 ; 0.04 ;-0.04 ; 0.04 ;-0.04 ; \ldots ; 0.04 ;-$ 0.04 ; ] resultou na fig. 6.1 .

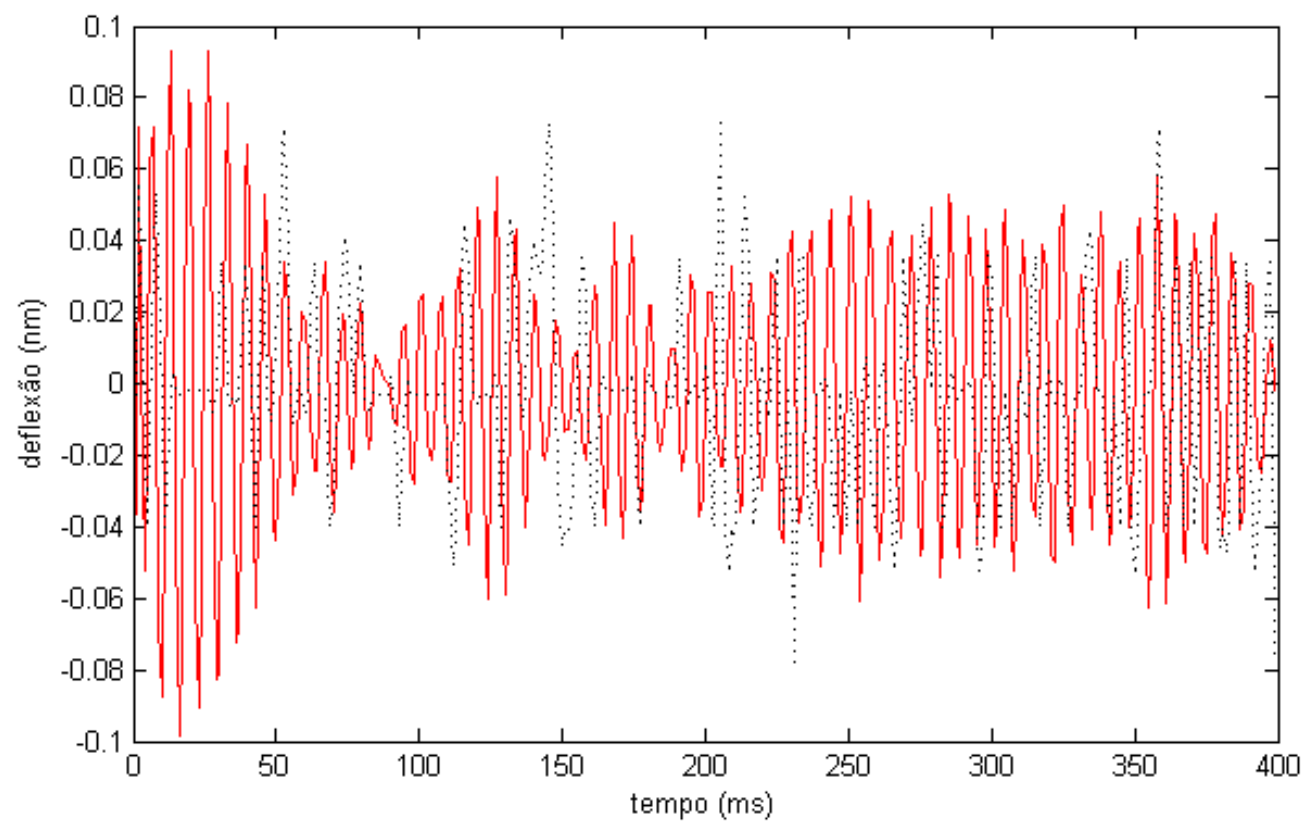

Figura 6. 1- Em pontilhado preto a série temporal para $\mathrm{z}=537 \mathrm{~nm}$ e em vermelho a série identificada pelo método de espaço de estados.

A análise e obtenção das equações foram formuladas no software Matlab® na ferramenta Identification toolbox proposto por Ljung (1999) no modo espaço de estados. 
Calculando $R^{2}=0.52$ e FIT $=53.28 \%$ fica caracterizado que não representam um bom índice, já que devem ficar acima de 0.9 e $90 \%$ respectivamente. Na figura 6.1 muitos pontos não foram identificados e seguindo a recomendação padrão para identificação de sistemas algum outro modo deve ser proposto, um segundo plano seria a mudança de série com novas entradas ou a alteração do experimento por completo.

O resultado mostrou o número livres de coeficiente $=28$, e parametrização de forma livre (Todos coeficientes em A,B,C livres). Muitas vezes, é mais fácil definir um modelo de espaço de estados com parâmetros em tempo contínuo, pois as leis físicas são mais frequentemente descrita em termos de equações diferenciais. Em tempo contínuo, a descrição de espaço de estado tem a seguinte forma:

$$
\begin{aligned}
& d x / d t=A x(t)+B u(t)+K e(t) \\
& y(t)=C x(t)+D u(t)+e(t)
\end{aligned}
$$

onde $\mathrm{A}, \mathrm{B}, \mathrm{C}$ e $\mathrm{D}$ são matrizes de estado-espaço. K é a matriz de perturbação. A $u(t)$ representa a entrada, $y(t)$ é a saída, $x(t)$ é o vector de estados e $e(t)$ é a perturbação.

$$
\begin{aligned}
& A=\begin{array}{ccccc}
x 1 & x 1 & x 2 & x 3 & x 4 \\
x 2 & 0.4584 & 1.562 & 0.6543 & -0.09272 \\
x 3 & -0.207 & -1.067 & -0.4395 & 0.4643 \\
x 4 & 0.07007 & 0.6008 & 0.09868 & -1.199 \\
x & & & &
\end{array}
\end{aligned}
$$

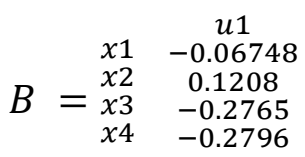

$$
\begin{aligned}
& C=\begin{array}{ccccc} 
& x 1 & x 2 & x 3 & x 4 \\
y 1 & 0.2929 & 0.05457 & -0.05066 & -0.006891
\end{array} \\
& D=\quad \begin{array}{l}
u 1 \\
y 10
\end{array} \\
& K=\begin{array}{cc}
x 1 & y 1 \\
x 2 & 4.593 \\
x 3 & 3.637 \\
x 4 & 6.221
\end{array}
\end{aligned}
$$


Devido ao índice não aceitável para validação na identificação de sistemas para a série de deflexões do microscópio de força atômica observável na Fig. 6.1, foi seguido o esquema de identificação de sistemas proposto por Ljung (1999):

\section{ESQUEMA DE IDENTIFICAÇÃO DE SISTEMAS}

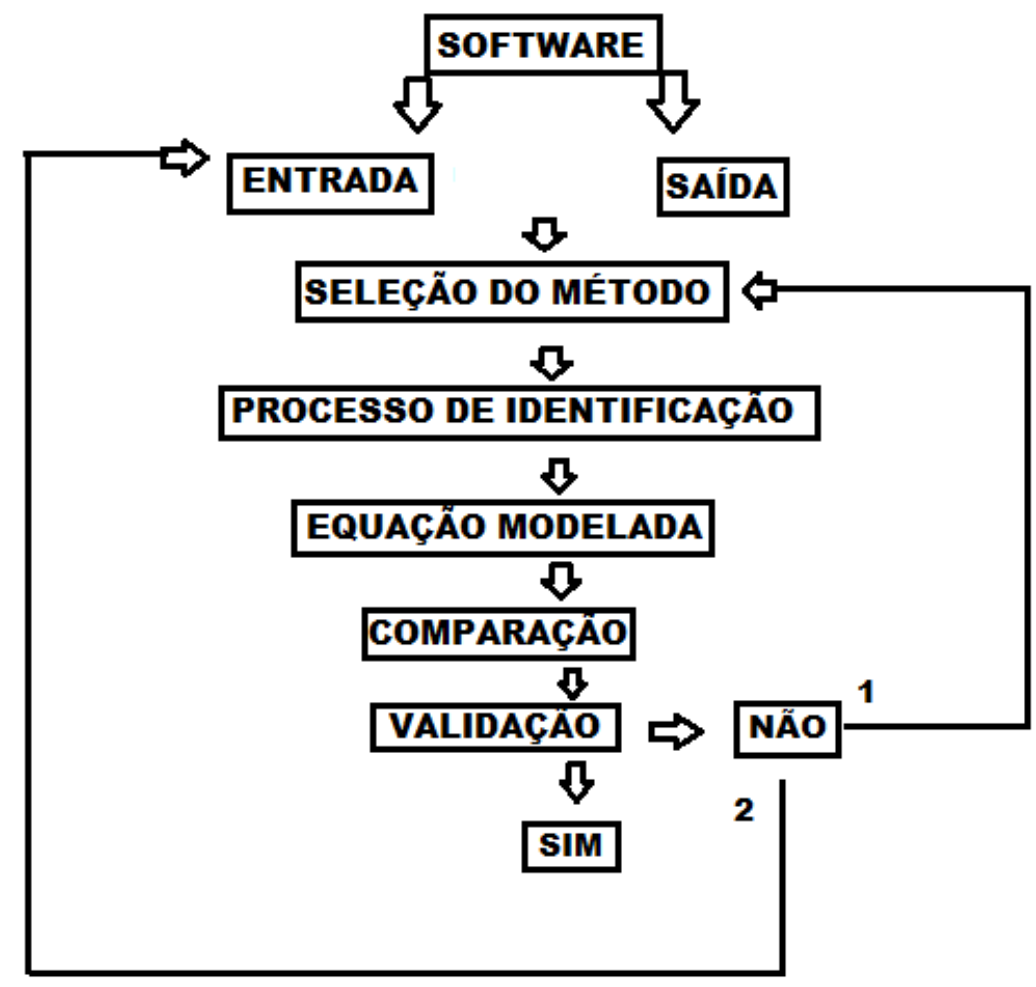

Figura 6. 2 - Esquema de identificação de sistemas (LJUNG, 1999).

A Fig. 6.2 mostra o esquema seguido para a identificação de sistemas de séries temporais. A primeira opção, a de trocar o método de identificação, não surgiu diferenças nos valores das validações. Na tabela 14, é mostrada a segunda opção, onde se utilizam as séries temporais do experimento $\mathrm{A}$, onde são caracterizadas com o índice de validação $\mathrm{R}^{2}$, a altura da microviga que caracteriza a série temporal e o valor do teste 0-1. 
Tabela 14 - Séries temporais e suas respectivas validações

\begin{tabular}{c|c|c|c|c|c|c|c|c}
\hline $\mathbf{Z}$ & $\mathbf{K}$ & $\mathrm{R}^{2}$ & $\mathbf{Z}$ & $\mathbf{K}$ & $\mathrm{R}^{2}$ & $\mathbf{Z}$ & $\mathbf{K}$ & $\mathrm{R}^{2}$ \\
\hline 10,2 & 0,99778 & 0,51 & 231,2 & 0,99236 & 0,51 & 452,2 & 0,99401 & 0,51 \\
27,2 & 0,99581 & 0,51 & 248,2 & 0,99485 & 0,52 & 469,2 & 0,99372 & 0,52 \\
44,2 & 0,99716 & 0,52 & 265,2 & 0,99277 & 0,52 & 486,2 & 0,9921 & 0,51 \\
61,2 & 0,99741 & 0,51 & 282,2 & 0,99528 & 0,53 & 503,2 & 0,99356 & 0,53 \\
78,2 & 0,99699 & 0,51 & 299,2 & 0,99589 & 0,51 & 520,2 & 0,99348 & 0,51 \\
95,2 & 0,99775 & 0,51 & 316,2 & 0,99179 & 0,51 & 537,2 & 0,99312 & 0,52 \\
112,2 & 0,99718 & 0,52 & 333,2 & 0,99318 & 0,52 & 554,2 & 0,99539 & 0,51 \\
129,2 & 0,99526 & 0,51 & 350,2 & 0,99368 & 0,52 & 571,2 & 0,99442 & 0,52 \\
146,2 & 0,99325 & 0,53 & 367,2 & 0,99359 & 0,53 & 588,2 & 0,99388 & 0,51 \\
163,2 & 0,99383 & 0,52 & 384,2 & 0,99262 & 0,51 & 605,2 & 0,99331 & 0,51 \\
180,2 & 0,99309 & 0,51 & 401,2 & 0,99429 & 0,52 & 622,2 & 0,99395 & 0,51 \\
197,2 & 0,99333 & 0,51 & 418,2 & 0,99641 & 0,51 & & & \\
214,2 & 0,99364 & 0,52 & 435,2 & 0,99766 & 0,51 & & & \\
\hline
\end{tabular}

A tabela 14 mostra valores muito baixos de validação e impulsionaram uma análise nos experimentos $\mathrm{B}, \mathrm{C}$ e $\mathrm{D}$, cujos resultados apresentaram-se não satisfatórios. Um quinto experimento 'E' feito com safira em meio líquido (água) houve um resultado satisfatório, conseguindo uma boa validação como mostrado na figura 6.3.

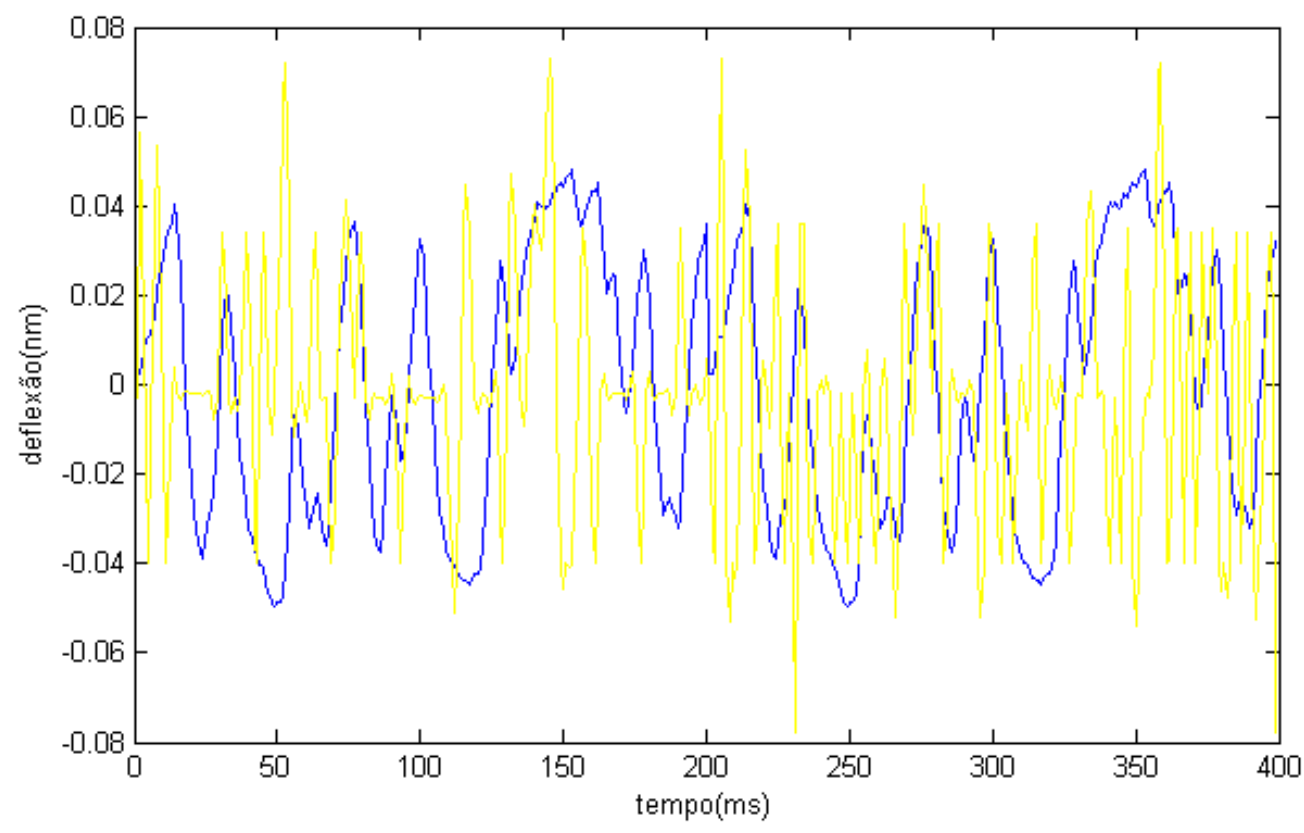

Figura 6. 3-Em cor azul a série temporal identificada com $\mathrm{z}=537.2 \mathrm{~nm}$ deflexão da microviga em meio líquido e em amarela a série com z=537.2 nm no ar 
Na figura 6.3 a representação gráfica em azul mostra o sistema dinâmico identificado com a série produzida em meio líquido e em cor amarela a série de deflexões de uma microviga em $\mathrm{z}=537.2 \mathrm{~nm}$. A série identificada operou em meio líquido $\left(\mathrm{H}_{2} \mathrm{O}\right)$, analisando a amostra de superfície de Safira, com constante elástica da microviga de $42 \mathrm{~N} / \mathrm{m}$, ponteira padrão retangular (standard), Sens. amp/sens de 100nm/V, amplitude setpoint de 178.85 $m V$, drive setpoint de $48.52 \mathrm{mV}$ e temperatura do laboratório de $22.1^{\circ} \mathrm{C}$, com entrada harmônica u $=[0.04 ;-0.04 ; 0.04 ;-0.04 ; 0.04 ;-0.04 ; 0.04 ;-0.04 ; . . ; 0.04 ;-0.04 ;]$ e teve como validação os valores Fit=91,2\% e $R^{2}=0,9548$ que representam valores aceitáveis para o modelo proposto.

Modo de identificação de sistema: Espaço de Estados no tempo contínuo

$$
\begin{aligned}
& d x / d t=A x(t)+B u(t)+K e(t) \\
& y(t)=C x(t)+D u(t)+e(t) \\
& A=\begin{array}{ccccc}
x 1 & -1.1 & -1.448 & 1.38 & x 4 \\
x 2 & 3.533 & -2.91 & 6.063 & -2.03 \\
x 3 & 4.452 & -15.48 & -13.7 & 11.34 \\
x 4 & 1.424 & -2.849 & -6.378 & -10.2
\end{array} \\
& B=\begin{array}{cc}
x 1 & 0.004924 \\
x 2 & -0.0728 \\
x 3 & -0.5834 \\
x 4 & -0.04361
\end{array} \\
& C=\begin{array}{cccc}
x 1 & x 2 & x 3 & x 4 \\
36.99 & 0.2903 & -0.3474 & -0.06767
\end{array} \\
& D=0 \\
& K=\begin{array}{ll}
x 1 & +0.2996 \\
x 2 & -0.1138 \\
x 3 & -0.2797 \\
x 4 & -1.1930
\end{array}
\end{aligned}
$$

Na figura 6.3 apresenta-se, também, a série temporal identificada com a microgiva operando em ar. Observa-se nesse caso que a amplitude nos pontos são maiores o que pode 
ter sido ocasionado por falha na identificação que desconsiderou a entrada de uma força altamente não linear (como a força de Van der Waals) ou por um erro no laser de detecção do AFM devido a alta velocidade de vibração. Na identificação de sistemas em meio líquido observou-se que a velocidade e amplitude diminuem e a o período aumenta (ver as regiões da figura 6.3).

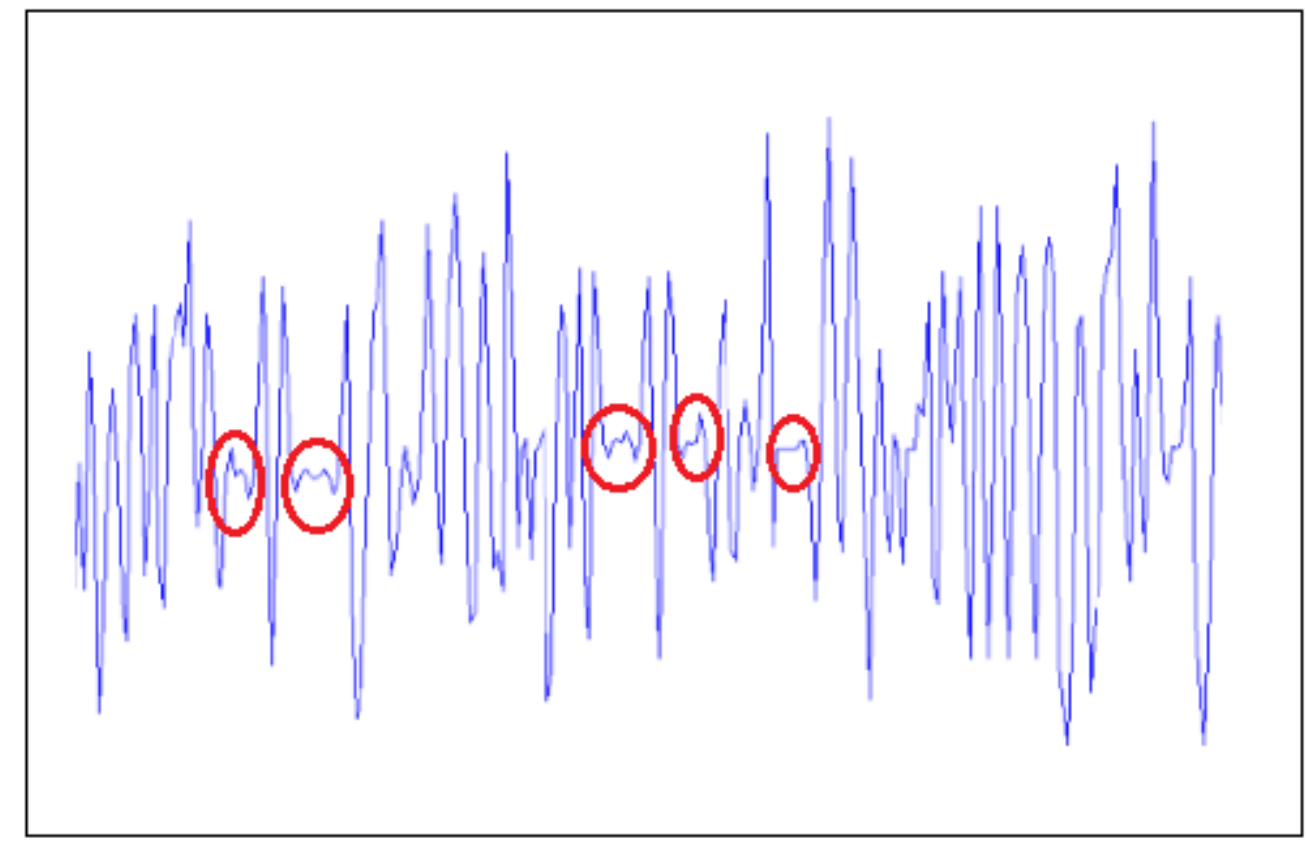

Figura 6. 4 - Exemplo de região da série temporal onde não há identificação de sistemas

A fig. 6.4 apresenta um exemplo de uma região de uma série temporal onde não há identificação de sistemas. As pequenas ondas apresentadas na fig. 6.4 (circuladas em vermelho) não apareceram nas séries temporais em meio líquido e por isso, provavelmente, seja mais fácil identificar sistemas na presença de meio líquido. 


\section{CAPÍTULO 7 \\ CONCLUSÕES}

Na presente tese foi apresentado um modelo matemático para modelar a microviga do microscópio de força atômica que considera as não linearidades da rigidez da microviga e as forças de Van der Waals. O modelo foi adimensionalizado e mapeado em espaço de estados. Considerando uma série temporal obtida da equação diferencial obteve-se o tempo de defasagem (por meio da informação mútua) e a dimensão de imersão (por meio dos falsos vizinhos próximos) para a reconstrução do espaço de estados utilizando o trabalho de Takens (1991). Esse mesmo procedimento foi utilizado nas séries temporais medidas experimentalmente. O objetivo dessa tese foi de identificar situações caóticas através da dinâmica da microviga do AFM investigada por meio de séries temporais obtidas de experimentos. Para tanto, foram feitos testes práticos para a obtenção de dados na forma de séries temporais. Esses testes foram realizados no Laboratório do Instituto de Física da USP São Carlos com o AFM trabalhando em cinco configurações distintas em que se varia o tipo de amostra (borracha ou safira), a geometria da ponteira (triangular ou retangular), e o meio de operação (ar, água e nitrogênio). Assim, por meio da realização dos testes experimentais foram obtidas diversas séries temporais do AFM operando em um único ponto e em uma trajetória retilínea. Existem na literatura diversas pesquisas que utilizam séries temporais para identificar e prever o comportamento de sistemas dinâmicos. Essas pesquisas utilizam diversos métodos, tais como: reconstrução de espaço de estado; identificação de sistemas; análise de caos por meio de escala indexada; entre outros mecanismos. É importante ressaltar que no trabalho desenvolvido foram utilizadas técnicas de análise de séries temporais não lineares e de identificação de sistemas para detectar e caracterizar não linearidades estruturais em séries temporais de um microscópio de força atômica obtidas experimentalmente em diversas condições.

Na microscopia de força atômica há diversas variáveis que influenciam a dinâmica do movimento da microviga e, consequentemente, a ampliação da imagem, como: a altura em que a microviga vibra; a força de Van der Waals; a geometria da ponta da microviga; amplitude de vibração; o coeficiente de amortecimento devido ao ar, a água, ou nitrogênio 
entre a microviga e a amostra; a constante elástica da microviga; a massa da microviga, entre outras variáveis.

Os experimentos foram realizados com o AFM trabalhando em modo 'soft-tapping' nas condições de não-contato e contato. Primeiramente, utilizou o AFM percorrendo a superfície do material ao longo de uma trajetória retilínea. Esse procedimento serviu para verificar o funcionamento do AFM e, também, obter imagens das superfícies. Em seguida utilizou-se o AFM fazendo a leitura dos dados em um ponto fixo. Esses últimos resultados foram utilizados para a análise do comportamento dinâmico AFM e, no presente trabalho, foram denominados de séries temporais 'a', 'b', 'c' e 'd'.

Após a realização dos testes experimentais, diversas técnicas não-lineares foram aplicadas nas séries temporais coletadas. Com essas técnicas obteve-se o retrato de fase reconstruído de uma forma diferente das que usualmente são representadas por equações diferenciais massa mola, de espaço de estados ou de funções de transferência. A análise do da dinâmica do AFM e da determinação de movimento caótico é feito pelo método do teste 0-1 e pela escala indexada. Verifica-se que a quantidade de pontos das séries temporais dos experimentos 'a', 'b', c' e 'd' foram suficientes para a realização dos testes $0-1$ e da escala indexada, mas não suficiente para vários testes de predição. Nesses testes experimentais a microviga está sob um determinado ponto fixo da superfície da amostra. Com a variação das alturas de vibração os movimentos alteram o valor dos níveis de dinâmicas caóticas, de forma a identificar qual é a altura que ocorreu menos caos. A partir disto, forma-se um mapa de como a microviga se comporta em regiões caóticas, semi-periódicas, ou periódicas facilitando a análise do funcionamento de um AFM. Observa-se que esse resultado contribui para uma melhor operação do AFM e, também, de novas pesquisas baseadas em séries temporais do AFM.

Existem na literatura uma grande quantidade de técnicas para o desenvolvimento de predição para dados na forma de séries temporais, como por exemplo, os Métodos de Previsão para Modelos com Valor Constante, o Método de Previsão de Média Móvel, o Método de Previsão com Suavização Exponencial, o Método de Previsão com Suavização Exponencial de Holt-Winters, o Método não linear de Wiener e Hammerstein, entre outros. A aplicação de métodos de predição nos resultados obtidos não puderam prever o comportamento do sistema, provavelmente pela aleatoriedade e caos dos resultados, de forma que optou-se por não predizer os dados para uma análise em tempo futuro.

Na presente tese foi realizada a identificação de sistemas com o intuito de se chegar a uma equação de movimento do AFM baseada nos dados experimentais. O sistema foi 
caracterizado com uma entrada de excitação harmônica e com a saída a série temporal. Observa-se que a utilização da série em meio líquido obtém-se uma boa validação (acima de 90\%) devido à diminuição da amplitude e aumento de amortecimento. Com a utilização de séries em meio gasoso (ar, nitrogênio), mesmo variando as configuração, não foram obtidos bons resultados de validação. No caso da amostra em meio gasoso nota-se uma maior dificuldade na aquisição de dados, pois a velocidade de vibração da microviga é extremamente rápida e isso pode ter afetado a identificação de sistemas em espaço de estados. Há, também, a possibilidade de que alguns pontos não foram capturados pelo laser, e não entraram no processo de identificação resultando em imprecisão dessa análise. Para contornar esse problema, foram feitos vários e repetidos ensaios para minimizar possíveis erros. No caso da microviga vibrando em água, o período da onda é maior de forma que os erros são minimizados e foi possível obter uma boa validação no processo de identificação.

Retomando aos objetivos da presente tese, conclui-se que foi possível identificar, com a utilização da análise de séries temporais obtidas de experimentos, regiões de operação do AFM com maior e menor "teor" de comportamento caótico. O conhecimento dessas regiões é fundamental para um melhor funcionamento do AFM. Em trabalhos futuros, os mapas gerados pelo teste 0-1 devem ser explorados com uma maior quantidade de variáveis, pois já revelaram, nos resultados da presente tese, que existem regiões onde o microscópio ampliaria melhores as imagens que é o que se busca quando se está operando um microscópio de força atômica. 


\section{REFERÊNCIAS BIBLIOGRÁFICAS}

ABARBANEL, H. D. I. “Analysis of Observed Chaotic Data", Springer, New York (1996).

ABARBANEL, H. D. I.; BROWN, R.; SIDOROWICH, J.J.;TSIMRING, L. S. The analysis of observed chaotic data in physical systems, Rev. Mod. Phys. 65, 1331 (1993).

AGUIRRE, L. A., Introdução à Identificação de Sistemas - Técnicas Lineares e NãoLineares Aplicadas a Sistemas Reais, IBSN - 9788570415844 - Editora UFMG

AKHSHANI, A., AKHAVAN, A. , MOBARAKI, A., LIM, S.-C., HASSAN, Z., 2014, "Pseudo random number generator based on quantum chaotic map", Commun. Nonlinear Sci Numer Simulat, 19, p. 101 - 111.

ASHHAB, M., SALAPAKA M.V., DAHLEH M., MEZIC H I. Dynamical analysis and control of micromicrovigas. Automatica; 1999, 35: pp. 1663-1670.

ASHHAB, M.; SALAPAKA, M.V.; DAHLEH, M.; MEZIC, I. Melnikov-based dynamical analysis of micromicrovigas in scanning probe microscopy. Nonlinear Dyn 1999;20:197-220.

AWREJCEWICZ, J., KRYSKO, A. V., SOLDATOV, V., 2011, "On the wavelet transform application to a study of chaotic vibrations of the infinite length flexible panels driven longitudinally”, International Journal of Bifurcation and Chaos 11, 2011, 3347-3371.

BALTHAZAR, J. M., TUSSET, A. M., BUENO, A. M. Tm-afm nonlinear motion control with robustness analysis to parametric errors in the control signal determination. Journal of Theoretical and Applied Mechanics, v. 52, n. 1, p. 93-106, 2014.

BALThAZAR, J. M., TUSSET, A. M., SOUZA, S. L. T., BUENO, A. M., 2012, "Micromicroviga Chaotic Motion suppression in tapping mode atomic force microscope", Proc. Inst. Mech. Eng. C J. Mech. Eng. Sci., 227, p. 1730-1741.

BALTHAZAR, J.M., TUSSET, A.M., BUENO, A. M., RODRIGUES, B. P. J. (2012). On an Overview of Nonlinear and Chaotic Behavior and Their Controls of an Atomic Force Microscopy (AFM) Vibrating Problem, Nonlinearity, Bifurcation and Chaos - Theory and Applications, Prof. Jan Awrejcewicz (Ed.), InTech, DOI: 10.5772/51834. Available from: http://www.intechopen.com/books/nonlinearity-bifurcation-and-chaos-theory-andapplications/on-an-overview-of-nonlinear-and-chaotic-behavior-and-their-controls-of-anatomic-force-microscopy-af 
BALTHAZAR, J.M., TUSSET, A.M., DE SOUZA, S. L. T., BUENO, A. M., Micromicroviga chaotic motion suppression in tapping mode atomic force microscope. Proceedings of the institution of mechanical engineers part c-journal of me, v. 227, n. 8, p. 1730-1741, AUG 2013.

BEHNIA, S., ZIAEI, J., GHIASSI, M., 2013, “A New Approach to the Study of Heartbeat Dynamics Based on Mathematical Model”, Electrical Engineering (ICEE), 2013 21st Iranian Conference on. IEEE.

BENÍTEZ, R., BOLÓS, V. J., RAMÍREZ, M. E., 2010, “A wavelet-based tool for studying non-periodicity”, Computers and Mathematics with Applications, 60, p. 634-641.

BERGÉ, P.; POMEAU, Y.; VIDAL, C. “'Order Within Chaos: Towards a deterministic approach to turbulence", Wiley, New York (1986).

BERNARDINI, D.; REGA, G.; LITAK,G.; AND SYTA, A. (2013). Identification of regular and chaotic isothermal trajectories of a shape memory oscillator using the $0-1$ test. Proc. Inst. Mech. Eng. K J. Multi-body Dyn. 227: 17-22.

BERNARDINI, D; LITAK, G. D., (2015). An overview of 0-1 test for chaos. J. Brazilian Soc. Mech. Sci.:1-8.

BINNI NG, G., GERBER C., QUATE C. Atomic Force Microscope. Phys. Rev. Lett.; 1986; 56: pp. 930-933.

BOWEN, W. R.; HILAL, N. Microscopy in process engineering, na introdution to afm for improved processes and products, Elsevier; 2009 USA.

BRAGHIN, F., RESTA, F., LEO, E., SPINOLA, G., 2007, "Nonlinear dynamics of vibrating MEMS". Sens. Actuators A, Phys. 134, p. 98-108.

BUENO, A. M., BALTHAZAR, J. M., PIQUEIRA, J. R. C. Phase-locked loop application to frequency modulation - atomic force microscope. Proceedings of the 9th Brazilian Conference on Dynamics Control and their Applications Serra Negra, SP - ISSN 2178-3667

BUENO, A. M., BALthaZAR, J. M., PIQUEIRA, J. R. C. Simulations of the Frequency Modulated - Atomic Force Microscope (FM-AFM) Nonlinear Control System. Chaotic Modeling and Simulation (CMSIM) 1: 173-186, 2011

CASDAGLI, M. Chaos and deterministic versus stochastic nonlinear modeling, J. Roy. Stat. Soc. 54, 303 (1991).

CASDAGLI, M. Recurrence plots revisited, Physica D 108, 206 (1997). 
CASDAGLI, M.; EUBANK, S. "Nonlinear Modeling and Forecasting", Santa Fe Institute Studies in the Science of Complexity, Proc. Vol. XII, Addison-Wesley, Reading, MA (1992).

CASDAGLI, M; EUBANK, S.; FARMER, J.D.; GIBSON, J. State space reconstruction in the presence of noise, Physica D 51, 52 (1991).

CASDAGLI, M. Nonlinear prediction of chaotic time series, Physica D 35, 335 (1989);

CHANDRE, C. , WIGGINS, S., UZER, T., 2003, "Time-frequency analysis of chaotic systems", Physica D, 181, p.171-196.

CLAEYSSEN, J. R., TSUKAZAN, T., TONETTO, L., BALTHAZAR, J. M. Forced oscillations with continuum models of Atomic force microscopy. 9th International Conference on Mathematical Problems in Engineering, Aerospace and Sciences. AIP Conf. Proc. 1493, 230-237 (2012); doi: 10.1063/1.4765494. American Institute of Physics 978-07354-1105-0

DISTEFANO, N.; RATH, A. System identification in nonlinear structural seismic dynamics. Comput.Meth. Appl. Mech. Engng 5, 353-372 (1975).

EISENSCHITZ, E.; LONDON F. Zs.Phys.60, 491 (1930): Teoria Quântica para as Forças de van der Waals.

FARMER, J. D.; SIDOROWICH, J.J. Predicting chaotic time series. Phys. Rev. Lett. 59,845 .

FRASER, A. M.; SWINNEY, H. L. Independent coordinates for strange attractors from mútual information, Phys. Rev. A 33, 1134 (1986).

GARCIA, R.; SAN PAULO, A. Dynamics of a vibrating tip near or in intermittent contact with a surface. Physical Review B 61, 20, 2000

GOTTWALD, G.; MELBOURNE, I. (2004). A new test for chaos in deterministic systems. Proceedings of the Royal Society of London A 460: 603-611.

GOTTWALD, G.; MELBOURNE, I. Testing for chaos in deterministic systems with noise. Physica D 212: 100-110, 2005

GRAEFF, CARLOS (Org.). Nanotecnologia: ciência e engenharia. São Paulo: Cultura Acadêmica, 2012. (Coleção PROPe Digital - UNESP). ISBN 9788579833779. Disponível em: 〈http://hdl.handle.net/11449/123647>.

GRASSBERGER,P.; SCHREIBER, T.; SCHAFFRATH, C. Non-linear time sequence analysis, Int. J. Bifurcation and Chaos 1, 521 (1991). 
GUZZO, M. Fourier analysis of chaotic motions and applications to Celestial mechanics. http://dx.doi.org/10.1007/978-1-4020-4706-0_6

H. KANTZ, T. SCHREIBER "Nonlinear Time Series Analysis", Cambridge University Press, Cambridge (1997).

HANAGUD, S.V.; MEYAPPA, M.;. CRAIG, J. I. Method of multiple scales and identification of nonlinear structural dynamic systems. AIAA Paper 83-0813, $24^{\text {th }}$ Structures, Structural Dynamics, and Materials Conference, Lake Tahoe, Nevada, May 2-4, 1983.

HEGGER, R.; KANTZ, H.; SCHREIBER, T. Practical implementation of nonlinear time series methods: The TISEAN package, CHAOS 9, 413 (1999)

HEGGER, R.; KANTZ, H.. Embedding of sequences of time intervals, Europhys. Lett. 38, 267 (1997).

HISTÓRIA DA MICROSCOPIA. IN INFOPÉDIA [Em linha]. Porto: Porto Editora, 2003-2010. [Consult. 2010-10-12].

HORNSTEIN, S, GOTTLIEB O. Nonlinear dynamics, stability and control of the scan process in noncontacting atomic force microscopy. Nonlinear Dyn.; 2008; 54: pp. 93-122.

HU, S., RAMAN, A. (2006). Chaos in atomic force microscopy. Physical Review Letters, 96(3), 036107.

HU, S.; RAMAN, A. Chaos in atomic force microscopy. Phys Rev Lett 2006/96:036107.

HUTTER, J.L.; BECHHOEFER, J.; Calibration of atomic-force microscope tips. Rev Scientific instrum 1993;64(7):1868-73.

IBRAHIM, S.R. Time-domain quasilinear identification of nonlinear dynamic systems. AIAA Paper 83-0811, 24th Structures, Structural Dynamics, and Materials Conference, Lake Tahoe,

ILIUK, I., BALTHAZAR, J. M., TUSSET, A.M., BUENO, A. M., PIQUEIRA, J.R.C. Application of Wavelets to Characterize Dynamic Behavior of Energy Harvesting Systems (2013), Cobem, Ribeirão Preto, SP, Brazil

ILIUK, I., BALTHAZAR, J.M, TUSSET, A.M, PICCIRILLO, V., BRASIL, R.M.L.R.F., PIQUEIRA, J. R. C. ASME 2014 International Design Engineering Technical Conferences and Computers and Information in Engineering Conference. Volume 8: 26th Conference on Mechanical Vibration and Noise. Buffalo, New York, USA, August 17-20, 2014. ISBN: 978-0-7918-4641-4.Copyright @ 2014 by ASME 
ISRAELACHVILI, J. Intermolecular and Surface Forces - Academic Press, London (1995).

JALILI，N.; DADFARNIA，M.; DAWSON，D. M.; Distributed parameters base modeling and vibration analysis of micromicrovigas used in atomic force microscopy, Proceedings of the ASME 2009 International Design Engineering Technical Conferences \& Computers and Information in Engineering Conference, IDETC/CIE 2009, August 30 September 2, 2009, San Diego, California, USA

JALILI, N.; LAXMINARAYANA, K. A review of atomic force microscopy imaging systems: application to molecular metrology and biological sciences. Mechatronics 14:8, 907-945. 2004.

JOHANSSON, R., System modeling and identification, Prentice Hall, New Jersey, 1993;

KANTZ, H.; KURTHS, J.; MAYER-KRESS, G. “'Nonlinear analysis of physiological data", Springer, Berlin (1998).

KANTZ, H.; SCHREIBER, T, “Nonlinear Time Series Analysis". Cambridge University Press, Cambridge (1997).

KAPLAN, D.; GLASS, L. 'Understanding Nonlinear Dynamics", Springer, New York (1995).

KATOK, A.; HASSELBLATT, B. “'Introduction to the Modern Theory of Dynamical Systems", Cambridge University Press, Cambridge (1996).

KENNEL, M.B.; BROWN, R.; ABARBANEL, H.D.I. Determining embedding dimension for phase-space reconstruction using a geometrical construction, Phys. Rev. A 45, 3403 (1992).

LITAK, G., BERNARDINI, D., SYTA, A., REGA, G., RYSAK, A., 2013, “Analysis of chaotic non-isothermal solutions of thermomechanical shape memory oscillators", Eur. Phys. J. Special Topics, 222, p. 1637-1647.

LITAK, G.; SYTA, A.; WIERCIGROCH, M. (2009). Identification of chaos in a cutting process by the 0-1 test. Chaos, Solitons and Fractals 40: 2095-2101.

LIU, S., DAVIDSON, A., LIN, Q., 2004, "Simulation studies on nonlinear dynamics and chaos in a MEMS microviga control system”. J. Micromech. Microeng, 14, p. 10641073 .

LJUNG, L. "Modelos de Estado-Espaço". Seção 4.3 no Sistema de Identificação: Teoria para o usuário. 2a ed. Upper Saddle River, NJ: Prentice Hall, 1999, pp 93-102. 
LJUNG, L. System identification: Theory for the user, Prentice Hall, New Jersey, 1999 ;

LOZANO, J. R., GARCIA R. Theory of Multifrequency Atomic Force Microscopy. Physical Review Letters. PRL 100, 076102, 2008.

LOZANO, J.R., GARCIA R. Theory of Multifrequency Atomic Force Microscopy. Physical Review Letters; 2008; PRL 100: 076102.

LUO, A., WANG, F.Y.,2002, "Chaotic motion in a micro-electromechanical system with non-linearity from capacitors". Commun. Nonlinear Sci. Numer. Simul, 7, p, 31-49.

M. B. KENNEL, R. BROWN, AND H. D. I. ABARBANEL, Determining embedding dimension for phase-space reconstruction using a geometrical construction, Phys. Rev. A 45, 3403 (1992).

MALATKAR, P., NAYFEH, A. Parametric Identification Technique for SingleDegree-of-Freedom Weakly Nonlinear Systems with Cubic Nonlinearities. Journal of Vibration and Control March/April 2003 9: 317-336, doi: 10.1177/107754603030754

MASRI, S.R.; CAUGHEY, T.K. A nonparametric identification technique for nonlinear dynamic problems. J. Appl. Mech. 46,433-447 (1979).

MESTROM, R.M.C., FEY, R.H.B., VAN BEEK, J.T.M., PHAN, K.L., NIJMEIJER, H., 2007, "Modeling the dynamics of a MEMS resonator". Sens. Actuators A, Phys. 142., p. $6-15$

MOON, F.C., 1998, Applied Dynamics-With Applications to Multibody and Mechatronic Systems. Wiley-Interscience, New York.

NAYFEH, A. H. Introduction to Perturbation Techniques, Wiley, New York. 1981.

NAYFEH, A. H. On the low-frequency drumming of bowed structures. J. Sound Vibr. 94, 551-562 (1984).

NAYFEH, A. H. Perturbation Methods, USA: John Wiley e Sons, 1973.

NAYFEH, A. H. Random motion and dynamic response: parametric identification of nonlinear dynamics. Computers \& Structures Vol 20. No. 1-3, pp 487-493. 1985

NAYFEH, A. H.; MOOK, D. T.; Non Oscillations. (1979) ISBN 0-471 12142-8 John Willey e sons.

NAYFEH, A.H.; BALACHANDRAN, B. Applied Nonlinear Dynamics: Analytical, Computation and Experimental Methods. John Wiley and Sons, N.Y., 1995.

NOZAKI, R., BALTHAZAR, J.M., TUSSET, A.M., BUENO, A.M., PONTES, B.R. Nonlinear Control System Applied to Atomic Force Microscope Including Parametric 
Errors. Journal of Control, Automation and Electrical Systems. DOI: 10.1007/s40313-0130034-1

NOZAKI, R., NAVARRO, H. A., BRASIL, R, M. L. R. F., SILVA, M. A. P., TUSSET, A. M., BUENO, A. M., BALTHAZAR, J. M. Signals Generated by a Sensor That Captures the Microviga Deflection of the Atomic Force Microscope With Nonlinear Behavior. Volume 4A: Dynamics, Vibration, and Control. Montreal, Quebec, Canada, November 14-20, 2014. Conference Sponsors: ASME ISBN: 978-0-7918-4647-6. doi:10.1115/IMECE2014-38386

OTT, E.; SAUER, T.;YORKE, J. A. “Coping with Chaos", Wiley, New York (1994).

PACKARD, N.; CRUTCHFIELD, J.; FARMER, D.; SHAW, R. (1980). Geometry from a time series. Physical Review Letters 45:712-715

PAYAM, A.F.; FATHIPOUR, M.; YAZDANPANAH, M. J. High precision imaging for non-contact mode atomic force microscope using an adaptive nonlinear observer and output state feedback controller, Digest Journal of Nanomaterials and Biostructures Vol. 4, No.3, September 2009, p. 429-442.

PAYAM, AF., FATHIPOUR M, YAZDANPANAH MJ. High precision imaging for non-contact mode atomic force microscope using an adaptive nonlinear observer and output state feedback controller. Digest Journal of Nanomaterials and Biostructures; 2009; 4(3): pp. 429-442.

PERMANN, D. , HAMILTON, I.,1992, "Wavelet analysis of time series for the Duffing oscillator: The detection of order within chaos", Phys. Rev. Lett. 69, p. 2607 2610.

PICCIRILlO, V., BALTHAZAR, J. M., TUSSET, A. M., BERNARDINI, D., REGA, G., Characterizing the Nonlinear Behavior of a Pseudoelastic Oscillator via the Wavelet Transform, Proc. Inst. Mech. Eng. K J. Multi-body Dyn., accepted for publication.

R., HEGGER, H. KANTZ, AND T. SCHREIBER, Practical implementation of nonlinear time series methods: The TISEAN package, CHAOS 9, 413 (1999)

RAMAN A., MELCHER J, TUNG R. Microviga Dynamics in Atomic Force Microscope. Nanotoday; 2008; 3, 1-2: pp. 20-27.

RAMAN A.; MELCHER J.; TUNG R. Microviga Dynamics in Atomic Force Microscope. Nanotoday, 3, 1-2, 20-27, 2008.

RAMAN, A., MELCHER J, TUNG R. Microviga Dynamics in Atomic Force Microscope. Nanotoday; 2008; 3, 1-2: pp. 20-27. 
RICARDO, G., SAN PAULO, A. Dynamics of a vibrating tip near or in intermittent contact with a surface. Physical Review B; 2000; 61, 20.

RODRIGUES, K. S., BALTHAZAR, J.M., TUSSET, A.M., BUENO, A.M., PONTES, B.R. Preventing chaotic motion in tapping-mode atomic force microscope Journal of Control, Automation and Electrical Systems, 2014

RODRIGUES, K. S., BALTHAZAR, J.M., TUSSET, A.M., PONTES, B.R. On a control design to an afm micromicroviga beam, operating in a tapping-mode, with irregular behavior. 2011/1/1. ASME 2011 International Design Engineering Technical Conferences and Computers and Information in Engineering Conference

ROUKES, M., 2001. "Nanoelectromechanical systems face the future”. Phys. World, Vol. 14, p. 25-31.

RUETZEL, S.; LEE, S.I.; RAMAN, A. Nonlinear dynamics of atomic-forcemicroscope probes driven in Lennard-Jones potentials. Proc R Soc Lond A 2003;459:192548.

SAUER, T. Reconstruction of dynamical systems from interspike intervals. Phys. Rev. Lett. 72, 3811 - Published 13 June 1994

SAUER, T.; YORKE, J.; CASDAGLI, M. Embedology, J. Stat. Phys. 65, 579 (1991).

SAVI, M. A. (2006), "Dinâmica não-linear e caos", Editora E-papers

Savi, M. A. (2006), "Dinâmica não-linear e caos", Editora E-papers

SCHREIBER, T. Interdisciplinary application of nonlinear time series methods, Phys. Reports 308, 1 (1999).

SCHREIBER, T. AND SCHMITZ, A. Surrogate time series, Physica D 142346 (2000)

SCHREIBER, T. Efficient neighbor searching in nonlinear time series analysis, Int. J. Bifurcation and Chaos 5, 349 (1995).

SCHREIBER,T. Interdisciplinary application of nonlinear time series methods, Phys. Reports 308, 1 (1999).

SCHREIBER,T.; SCHMITZ, A.; Surrogate time series, Physica D 142346 (2000)

SCHUSTER, H. G. “'Deterministic Chaos: An introduction". Physik Verlag, Weinheim (1988).

SEBASTIAN, A.; GANNEPALII, A.; GANNEPALI, M. V.; SALAPKA, M. V. A review of the systems approach to the analysis of dynamic-mode atomic force microscopy. IEEE Transactions on Control Systems Technology, 15(15), 952- 959 (2007). 
SEBASTIAN, R., LEE SL, RAMAN A. Nonlinear dynamics of atomic-forcemicroscope probes driven in Lennard -Jones potentials. Proc. R. Soc. Lond.; 2003; 459: pp. 1925-1948.

SINHA, A. Nonlinear dynamics of atomic force microscope with PI feedback. Journal of Sound and Vibration; 2005; 288: pp.387-394.

SINHA, N.K.; KUSZTA, B., Modeling and identification of dynamic systems, Van Nostrand, Reinhold Co., New York, 1983.

SLATER, J. C. Quantum Theory of Molecules and Solids. McGraw-Hill,1963. Biblioteca do CBPF: 530.4 s631

SOUZA, S. L. T., CALDAS, I.L., VIANA, R. L., BALTHAZAR, J. M., 2005, “Basins of attraction changes by amplitude constraining of oscillators with limited power supply", Chaos Solitons Fractals, 26, p. 1211-1220.

STROGATZ, S. H.,1994, Nonlinear Dynamics and Chaos: With Applications to Physics, Biology, Chemistry and Engineering" Addison-Wesley.

TAKENS, F. "Detecting Strange Attractors in Turbulence", Lecture Notes in Math. Vol. 898, Springer, New York (1981).

TORRENCE, C. AND COMPO, G.P. 1998. "A practical guide to wavelet analysis". Bulletin of the American Meteorological

TUSSET, A.M., BALTHAZAR, J.M., BASSINELlO, D.G., PONTES JR., B.R., FELIX,J L P., 2012, "Statements on chaos control designs, including a fractional order dynamical system, applied to a "MEMS" comb-drive actuator". Nonlinear Dynamics, 69, p. $1837-1847$.

UDWADIA, F. E.; AND KUO C.P., Nonparametric identification of a class of nonlinear lose-coupled dynamic systems. Earthquake Engng. Struct. Dyn. 9, 385-409 (1981).

WANG, C. C. ET AL. Chaos control in AFM system using sliding mode control by backstepping design. Communications in Nonlinear Science Numerical Simulation (2009), doi:10.1016/j.cnsns.2009.04.031

WEIGEND, A.S.; GERSHENFELD, N. A. “Time Series Prediction: Forecasting the future and understanding the past", Santa Fe Institute Studies in the Science of Complexity, Proc. Vol. XV, Addison-Wesley, Reading, MA (1993).

WOLF, A.; SWIFT, J.B.; SWINNEY, H.L.; VASTANO, J.A. Determining Lyapunov exponents from a Time Series, Physica D, Vol. 16, p. 285-315. 1985. 
WONG, L.A. AND CHEN, J.C., 2001. "Nonlinear and chaotic behavior of structural system investigated by wavelet transform techniques". International Journal of Non-linear Mechanics, Vol. 36, pp. 221-235.

WONG, L.A. AND CHEN, J.C., 2001. "Nonlinear and chaotic behavior of structural system investigated by wavelet transform

XIE, H., FEDDER, G., 2002, "Vertical comb-finger capacitive actuation and sensing for coms-MEMS”. Sens. Actuators A, Phys. Vol. 95, p. 212-221.

YABUNO, H. (2008). Stabilization and utilization of nonlinear phenomena based on bifurcation control for slow dynamics. Journal of Sound and Vibration, 315(3), 766-780.

YOUNIS, M.I., NAYFEH, A.H., 2003, "A study of the nonlinear response of a resonant microbeam to an electric actuation”. Nonlinear Dynamics, 31, p. 91-117.

YUN, C. B., AND SHINOZUKA,M. Identification of nonlinear structural dynamic systems. f. Struct. Mech. 8, 187-203 (1980).

ZANETTE, S. I.; Funcionamento de um microscópio de força atômica. Notas do curso ministrado no CBPF,1997. DCP/Centro Brasileiro de Pesquisas Físicas/MCT. 


\section{APÊNDICE}

\section{PROGRAMAS}

Programa que organiza as séries temporais em matrizes onde a linha é deflexão em contanto e em coluna a deflexão em modo 'soft tapping'.

clear all

$\mathrm{ClC}$

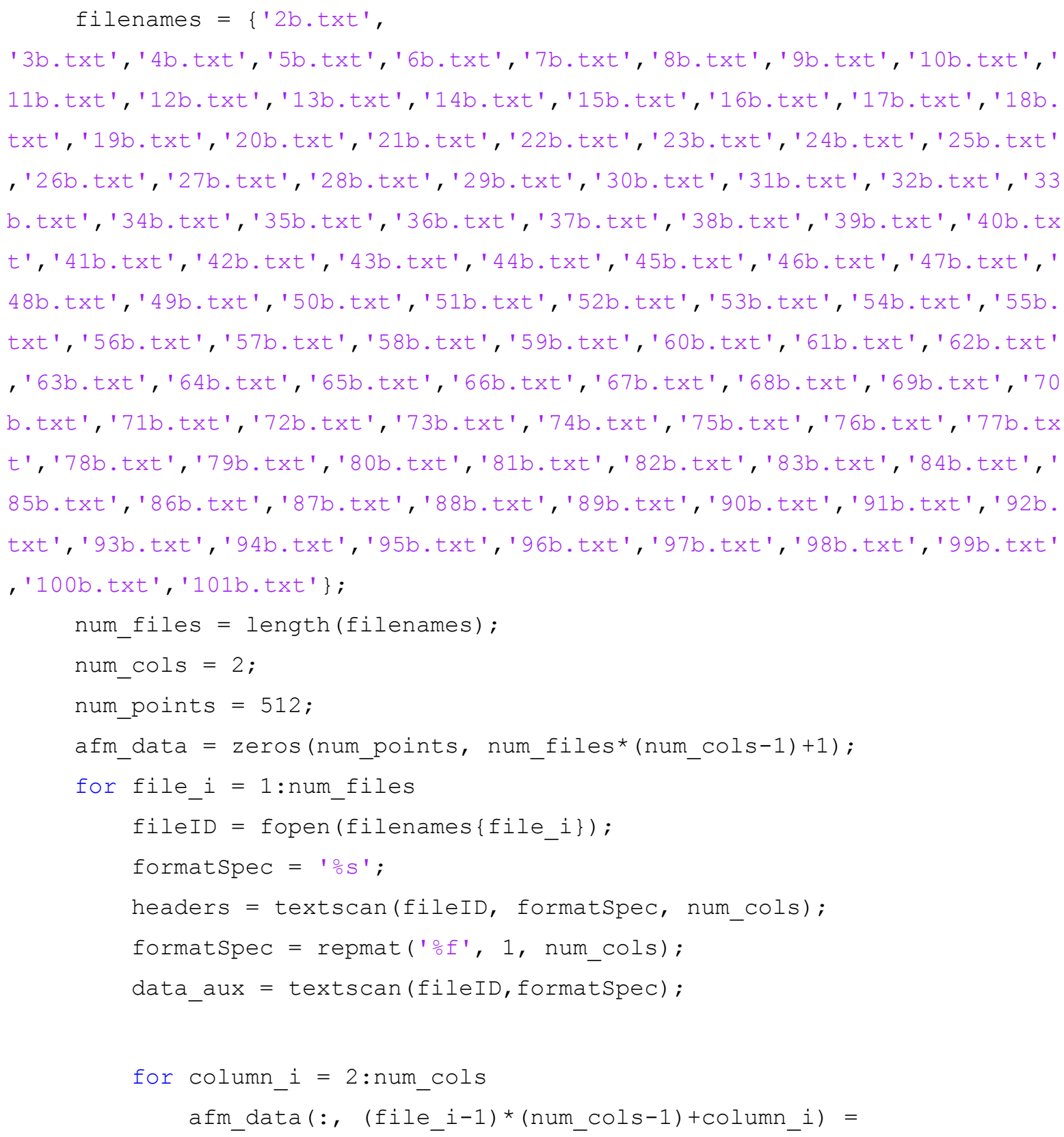




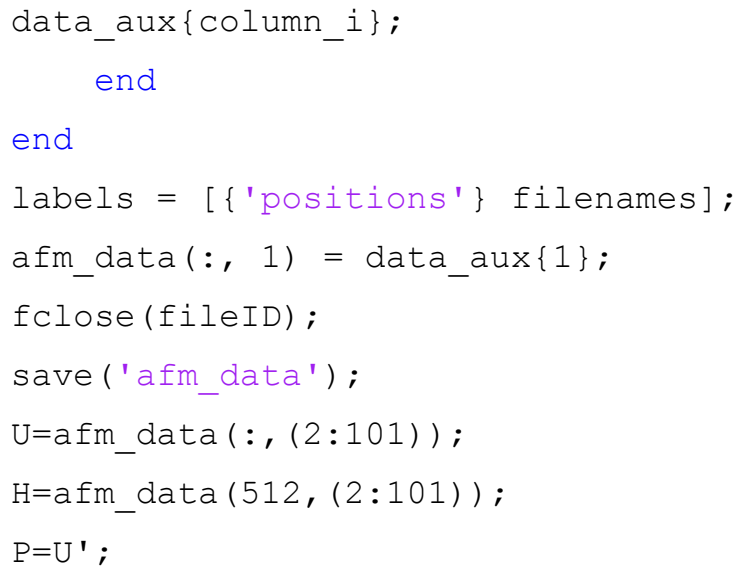

\section{SÉRIE TEMPORAL}

Uma pequena série temporal com $\mathrm{z}=620 \mathrm{~nm}$,

Scan size - zero

Constante elástica da microviga $=42 \mathrm{~N} / \mathrm{m}$

Tip padrão ( standard)

Sens. amp/sens $=100 \mathrm{~nm} / \mathrm{V}$

Amplitude setpoint $=178,85 \mathrm{mV}$

Drive setpoint $=48,52 \mathrm{mV}$

Temperatura $=22,1^{\circ} \mathrm{C}$

$\mathrm{A}=[$

$-1.1300000 \mathrm{e}-01 \quad-7.4000000 \mathrm{e}-02 \quad-1.5000000 \mathrm{e}-01 \quad-1.1200000 \mathrm{e}-01 \quad-7.4000000 \mathrm{e}-02$ $-1.5000000 \mathrm{e}-01 \quad-1.1200000 \mathrm{e}-01 \quad-1.5000000 \mathrm{e}-01 \quad-7.4000000 \mathrm{e}-02 \quad-1.5000000 \mathrm{e}-01$ $-1.1200000 \mathrm{e}-01-1.5000000 \mathrm{e}-01-1.5000000 \mathrm{e}-01-1.1300000 \mathrm{e}-01$ -

$1.1300000 \mathrm{e}-01 \quad-7.6000000 \mathrm{e}-02 \quad-1.5000000 \mathrm{e}-01 \quad-1.1300000 \mathrm{e}-01 \quad-1.1300000 \mathrm{e}-01$ $-7.6000000 \mathrm{e}-02 \quad-1.1300000 \mathrm{e}-01 \quad-1.5000000 \mathrm{e}-01-7.6000000 \mathrm{e}-02 \quad-1.1300000 \mathrm{e}-$ $01-1.1300000 \mathrm{e}-01 \quad-7.6000000 \mathrm{e}-02 \quad-7.5000000 \mathrm{e}-02 \quad-1.1300000 \mathrm{e}-01 \quad-$ $1.1300000 \mathrm{e}-01 \quad-7.6000000 \mathrm{e}-02 \quad-1.5000000 \mathrm{e}-01 \quad-1.5000000 \mathrm{e}-01 \quad-1.1300000 \mathrm{e}-01$ $-1.1300000 \mathrm{e}-01-1.1300000 \mathrm{e}-01 \quad-1.1300000 \mathrm{e}-01 \quad-7.6000000 \mathrm{e}-02 \quad-1.1300000 \mathrm{e}-$ $01-1.1300000 \mathrm{e}-01-1.1300000 \mathrm{e}-01 \quad-1.1300000 \mathrm{e}-01 \quad-1.5000000 \mathrm{e}-01 \quad-$ $1.1300000 \mathrm{e}-01 \quad-1.1300000 \mathrm{e}-01 \quad-1.5000000 \mathrm{e}-01 \quad-1.1200000 \mathrm{e}-01 \quad-1.5000000 \mathrm{e}-01$ $-1.1300000 \mathrm{e}-01-1.8800000 \mathrm{e}-01 \quad-1.5000000 \mathrm{e}-01 \quad-1.1300000 \mathrm{e}-01-1.1300000 \mathrm{e}-01$ $-1.1300000 \mathrm{e}-01 \quad-1.1300000 \mathrm{e}-01 \quad-1.5000000 \mathrm{e}-01 \quad-1.5000000 \mathrm{e}-01 \quad-1.1300000 \mathrm{e}-01$ $-1.1300000 \mathrm{e}-01-7.6000000 \mathrm{e}-02 \quad-1.1300000 \mathrm{e}-01 \quad-1.1300000 \mathrm{e}-01-1.1300000 \mathrm{e}-01$ $-1.5000000 \mathrm{e}-01-1.5000000 \mathrm{e}-01 \quad-1.5000000 \mathrm{e}-01 \quad-1.1200000 \mathrm{e}-01-1.1200000 \mathrm{e}-01$ $-1.5000000 \mathrm{e}-01-1.1300000 \mathrm{e}-01 \quad-1.1200000 \mathrm{e}-01 \quad-1.1200000 \mathrm{e}-01 \quad-7.4000000 \mathrm{e}-$ $02-1.1200000 \mathrm{e}-01-1.1200000 \mathrm{e}-01-1.5000000 \mathrm{e}-01 \quad-1.1200000 \mathrm{e}-01-$ $1.5000000 \mathrm{e}-01-1.5000000 \mathrm{e}-01-1.1200000 \mathrm{e}-01-1.5000000 \mathrm{e}-01 \quad-1.8800000 \mathrm{e}-01$ $-1.1200000 \mathrm{e}-01 \quad-1.8800000 \mathrm{e}-01 \quad-1.1200000 \mathrm{e}-01 \quad-1.1200000 \mathrm{e}-01 \quad-1.5000000 \mathrm{e}-01$ $-1.5000000 \mathrm{e}-01 \quad-1.1200000 \mathrm{e}-01-1.5000000 \mathrm{e}-01-1.5000000 \mathrm{e}-01 \quad-1.5000000 \mathrm{e}-$ $01-1.1200000 \mathrm{e}-01-1.1200000 \mathrm{e}-01-1.1200000 \mathrm{e}-01-1.1200000 \mathrm{e}-01$ 
$\begin{array}{llllll}1.5000000 \mathrm{e}-01 & -1.1200000 \mathrm{e}-01 & -1.5000000 \mathrm{e}-01 & -1.5000000 \mathrm{e}-01 & -1.1200000 \mathrm{e}-01 \\ -1.5000000 \mathrm{e}-01 & -1.5000000 \mathrm{e}-01 & -7.5000000 \mathrm{e}-02 & -1.5000000 \mathrm{e}-01 & -1.5000000 \mathrm{e}-01 \\ -1.5000000 \mathrm{e}-01 & -1.1200000 \mathrm{e}-01 & -1.1200000 \mathrm{e}-01 & -1.5000000 \mathrm{e}-01 & -1.1200000 \mathrm{e}-01 \\ -1.1200000 \mathrm{e}-01 & -1.1200000 \mathrm{e}-01 & -1.1200000 \mathrm{e}-01 & -1.1200000 \mathrm{e}-01 & -1.5000000 \mathrm{e}-01 \\ -1.5000000 \mathrm{e}-01 & -1.1200000 \mathrm{e}-01 & -1.1200000 \mathrm{e}-01 & -1.8800000 \mathrm{e}-01 & -1.5000000 \mathrm{e}-01 \\ -1.1200000 \mathrm{e}-01 & -1.5000000 \mathrm{e}-01 & -1.5000000 \mathrm{e}-01 & -1.5000000 \mathrm{e}-01 & -1.1200000 \mathrm{e}-01 \\ -1.5000000 \mathrm{e}-01 & -1.5000000 \mathrm{e}-01 & -1.5000000 \mathrm{e}-01 & -1.5000000 \mathrm{e}-01 & -1.5000000 \mathrm{e}-01 \\ -1.1200000 \mathrm{e}-01 & -1.1200000 \mathrm{e}-01 & -1.5000000 \mathrm{e}-01 & -1.5000000 \mathrm{e}-01 & -1.1200000 \mathrm{e}-01 \\ -1.5000000 \mathrm{e}-01 & -1.5000000 \mathrm{e}-01 & -1.1200000 \mathrm{e}-01 & -1.1200000 \mathrm{e}-01 & -1.5000000 \mathrm{e}-01 \\ -1.5000000 \mathrm{e}-01 & -1.1200000 \mathrm{e}-01 & -1.5000000 \mathrm{e}-01 & -7.5000000 \mathrm{e}-02 & -1.1200000 \mathrm{e}-01 \\ -1.1200000 \mathrm{e}-01 & -1.5000000 \mathrm{e}-01 & -1.8800000 \mathrm{e}-01 & -1.5000000 \mathrm{e}-01 & -1.1200000 \mathrm{e}-01 \\ -1.1200000 \mathrm{e}-01 & -1.5000000 \mathrm{e}-01 & -1.1200000 \mathrm{e}-01 & -1.1200000 \mathrm{e}-01 & -1.1200000 \mathrm{e}-01 \\ -1.8800000 \mathrm{e}-01 & -1.1200000 \mathrm{e}-01 & -1.1200000 \mathrm{e}-01 & -1.5000000 \mathrm{e}-01 & -1.5000000 \mathrm{e}-01 \\ -1.1200000 \mathrm{e}-01 & -1.5000000 \mathrm{e}-01 & -1.5000000 \mathrm{e}-01 & -1.1300000 \mathrm{e}-01 & -1.5000000 \mathrm{e}-01 \\ -1.5000000 \mathrm{e}-01 & -7.5000000 \mathrm{e}-02 & -1.1200000 \mathrm{e}-01 & -1.5000000 \mathrm{e}-01 & -1.1300000 \mathrm{e}-01 \\ -1.5000000 \mathrm{e}-01 & -1.1300000 \mathrm{e}-01 & -1.1300000 \mathrm{e}-01 & -1.5000000 \mathrm{e}-01 & -1.5000000 \mathrm{e}-01 \\ -1.5000000 \mathrm{e}-01 & -1.1300000 \mathrm{e}-01 & -1.5000000 \mathrm{e}-01 & -1.1300000 \mathrm{e}-01 & -1.1300000 \mathrm{e}-01 \\ -1.5000000 \mathrm{e}-01 & -1.5000000 \mathrm{e}-01 & -1.1200000 \mathrm{e}-01 & -1.5000000 \mathrm{e}-01 & -1.1300000 \mathrm{e}-01 \\ -1.8700000 \mathrm{e}-01 & -1.5000000 \mathrm{e}-01 & -1.5000000 \mathrm{e}-01 & -1.1200000 \mathrm{e}-01 & -1.5000000 \mathrm{e}-01 \\ -1.5000000 \mathrm{e}-01 & -1.5000000 \mathrm{e}-01 & -1.8800000 \mathrm{e}-01 & -1.5000000 \mathrm{e}-01 & -1.1300000 \mathrm{e}-01 \\ -1.5000000 \mathrm{e}-01 & -1.5000000 \mathrm{e}-01 & -1.1200000 \mathrm{e}-01 & -1.5000000 \mathrm{e}-01 & -2.2600000 \mathrm{e}-01]\end{array}$ 